\title{
Surfactants and digital alloys for strain relief in III-nitride distributed Bragg reflectors and related heterostructures via metal organic vapor phase epitaxy
}

\author{
Lee Ellen Rodak \\ West Virginia University
}

Follow this and additional works at: https://researchrepository.wvu.edu/etd

\author{
Recommended Citation \\ Rodak, Lee Ellen, "Surfactants and digital alloys for strain relief in III-nitride distributed Bragg reflectors \\ and related heterostructures via metal organic vapor phase epitaxy" (2011). Graduate Theses, \\ Dissertations, and Problem Reports. 4775. \\ https://researchrepository.wvu.edu/etd/4775
}

This Dissertation is protected by copyright and/or related rights. It has been brought to you by the The Research Repository @ WVU with permission from the rights-holder(s). You are free to use this Dissertation in any way that is permitted by the copyright and related rights legislation that applies to your use. For other uses you must obtain permission from the rights-holder(s) directly, unless additional rights are indicated by a Creative Commons license in the record and/ or on the work itself. This Dissertation has been accepted for inclusion in WVU Graduate Theses, Dissertations, and Problem Reports collection by an authorized administrator of The Research Repository @ WVU. For more information, please contact researchrepository@mail.wvu.edu. 
SURFACTANTS AND DIGITAL ALLOYS FOR STRAIN RELIEF IN III-

NITRIDE DISTRIBUTED BRAGG REFLECTORS AND RELATED

HETEROSTRUCTURES VIA METAL ORGANIC VAPOR PHASE EPITAXY

by

Lee Ellen Rodak

BSEE, BSCpE

Dissertation submitted to the College of Engineering and Mineral Resources

at West Virginia University

in partial fulfillment of the requirements

for the degree of

Doctor of Philosophy

in

Electrical Engineering

Approved by

Dimitris Korakakis, PhD, Committee Chairperson

Jeremy Dawson, PhD

Lawrence Hornak, PhD

Charter Stinespring, PhD

Nianqiang Wu, PhD

Lane Department of Computer Science and Electrical Engineering

Morgantown, West Virginia

2011

Keywords: Distributed Bragg Reflectors, Metal Organic Vapor Phase Epitaxy, Indium Surfactant, Digital Alloy

Copyright 2011 Lee Ellen Rodak 


\title{
A B S T R A C T
}

\section{Surfactants and Digital Alloys for Strain Relief in III-Nitride Distributed Bragg Reflectors and Related Heterostructures via Metal Organic Vapor Phase Epitaxy}

\author{
Lee Ellen Rodak
}

III-Nitride based semiconductors have emerged as one of the promising materials for electronic and opto-electronic devices, including but not limited to, solid state emitters, photodetectors, and transistors. Despite commercial success, several issues ranging from material growth to device fabrication remain unresolved and continue to hinder the efficiency of these devices. One such issue includes strain management in III-Nitride heterostructures. The binary alloys in the (Al,In,Ga)N family are characterized by a large lattice and thermal mismatch which leads to defect formation and cracking within heterostructures. These defects are detrimental to device fabrication and operation. This work investigates growth based techniques to manage strain in III-Nitride heterostructures and thereby reduce defect formation.

In particular, this work focuses on the development surfactant assisted growth and digital alloys as strain relieving techniques to minimize cracking in Aluminum Gallium Nitride $\left(\mathrm{Al}_{x} \mathrm{Ga}_{1 \text { - }}\right.$ ${ }_{x} \mathrm{~N}$ ) alloys and related heterostructures via Metal Organic Vapor Phase Epitaxy. Indium has been investigated as a surfactant in the growth of AlN/GaN Distributed Bragg Reflectors (DBRs) and has been shown to reduce the cracking by a factor of two. Using variable temperature x-ray diffraction studies, indium has been shown to influence the thermal expansion coefficients of the AlN layers. The digital growth technique has been investigated as a viable method for achieving high quality, crack free $\mathrm{Al}_{x} \mathrm{Ga}_{1-x} \mathrm{~N}$ films. Alloys with an AlN mole fraction ranging from 0.1 to 0.9 have been grown by adjusting the periodicity of these short period superlattice structures. High resolution x-ray diffraction has been used to determine the superlattice period along with the $a$ - and $c$-lattice parameter of the structure. High aluminum content digital $\mathrm{Al}_{x} \mathrm{Ga}_{1 \text { - }}$ ${ }_{x} \mathrm{~N}$ alloys have been employed in DBRs for high reflectivity, $>94 \%$, crack-free structures. The characterization of these structures via scanning electron microscopy, atomic force microscopy, and x-ray diffraction is presented along with the results from the integration of the DBR with visible wavelength LEDs. 


\section{A C K N O W L D G E M E N T}

I would genuinely like to thank the individuals who have assisted me in completing the work presented in this dissertation.

First and foremost, I would like to express my sincere gratitude to my advisor, Dr. Dimitris Korakakis, for his technical input, patient discussions, and guidance while I was completing this work. It is with his mentoring and encouragement that I was able to achieve this degree and the many accomplishments during my graduate studies.

I would additionally like to thank my committee members, Dr. Lawrence Hornak, Dr. Jeremy Dawson, Dr. Charter Stinespring, and Dr. Nick Wu for their input and technical discussions. I would specifically like to acknowledge Dr. Hornak and Dr. Dawson for their optical expertise and insightful input on the DBR characterization. I would like to thank Dr. Stinespring for his discussions on growth and Dr. Wu for his input on sample characterization.

Finally, I would like to acknowledge my lab colleagues, both past and present, for investing their time and effort into various parts of this work and the lab in general. I sincerely thank each and every one of the following people:

Dr. Kyoungnae Lee, Dr. Sridhar Kuchibhatla, Vamsi Kumbham, Joshua Justice, Justin Peacock, Vishal Narang, Anand Kadiyala, Benjamin Bearce, Bashar Hamza, Ronak Rahimi, Kenny Hite, Srinitya Musunuru, Rohit Goswami, Srikanth Raghavan, Christopher Miller, Wen-yu Chiang, Jin Wang, Dr. Ting Liu, Kalyan Kasarala, Nathan Berry Ann, Nanying Yang, Joshua Nightingale, John Harman, Richard Farrell, Nick Shelton, and Hyma Yalamanchili 


\section{TABLE OF CONTENTS}

ABSTRACT .ii

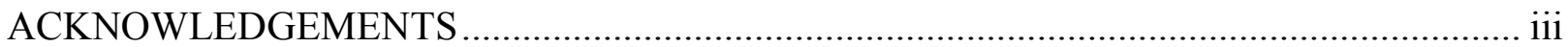

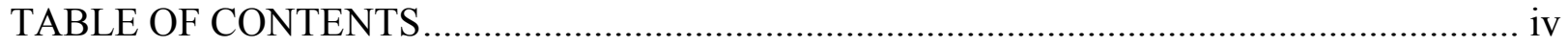

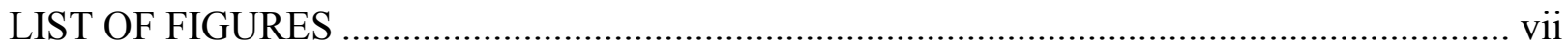

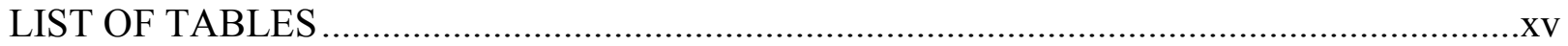

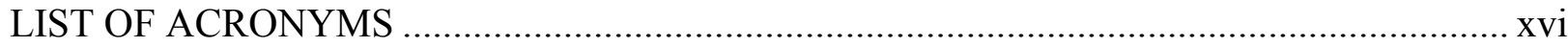

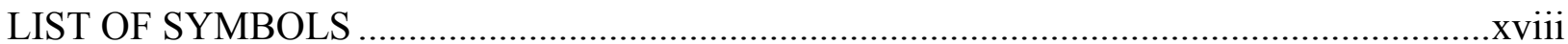

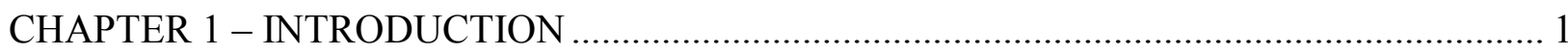

1.1 Statement of the Problem............................................................................................... 1

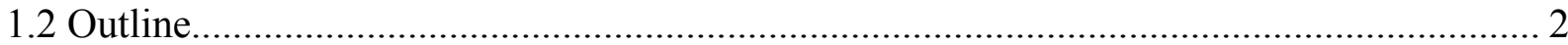

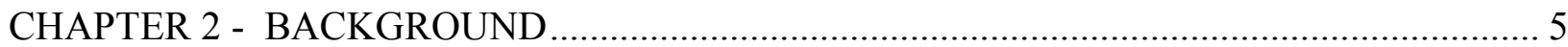

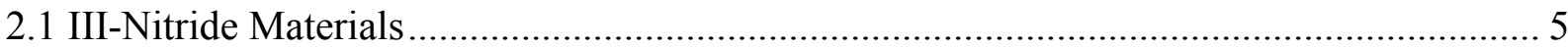

2.2 Material Growth via Metal Organic Vapor Phase Epitaxy (MOVPE) ……………......... 12

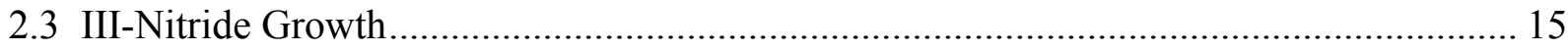

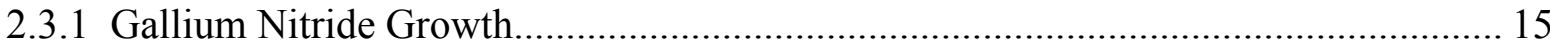

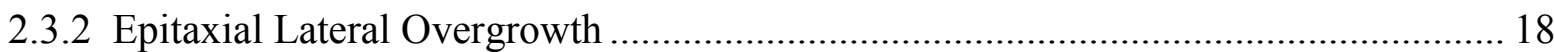

2.3.3 Aluminum Nitride and Aluminum Gallium Nitride Growth...................................... 22

2.3.4 Aluminum Nitride Interlayers ………................................................................... 23

2.3.5 Aluminum Gallium Nitride Graded Buffer Layers .................................................. 24

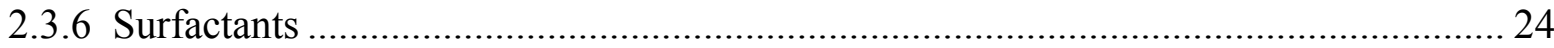

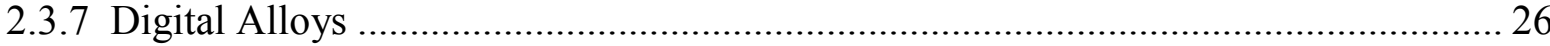

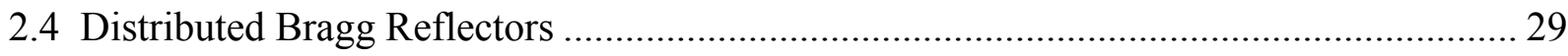

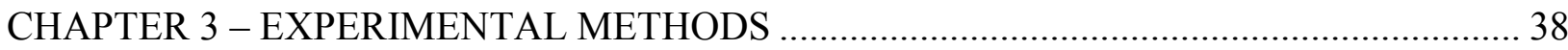

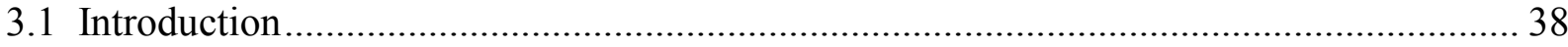

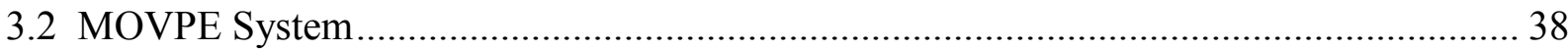

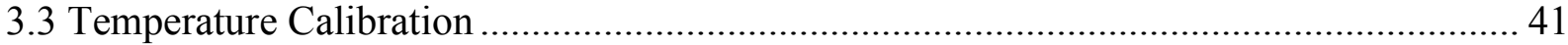

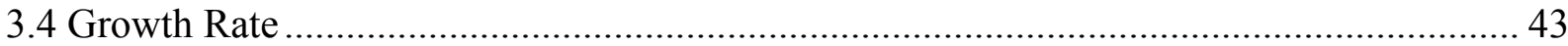

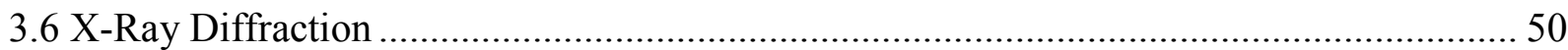




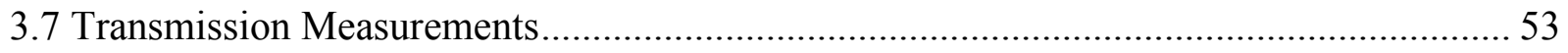

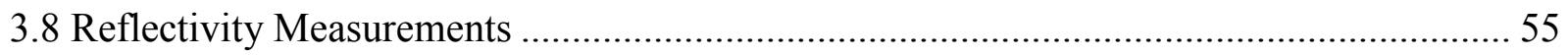

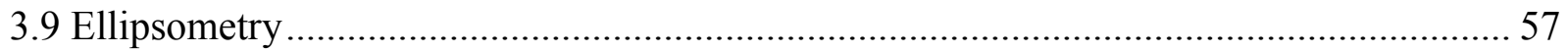

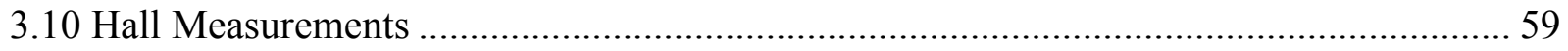

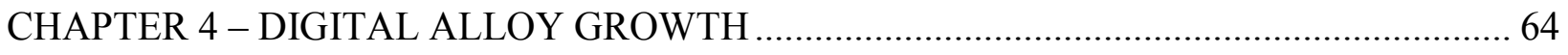

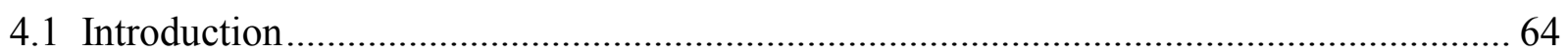

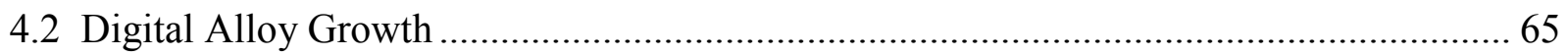

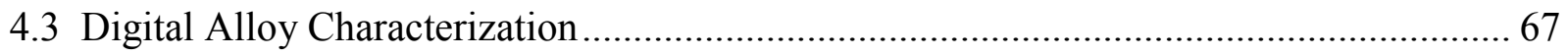

CHAPTER 5 - DISTRBUTED BRAGG REFLECTORS ……………………….................... 87

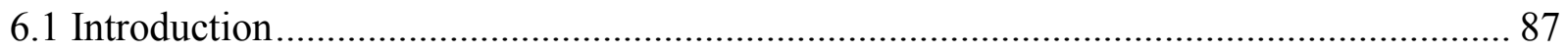

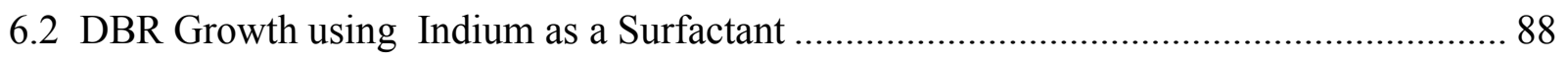

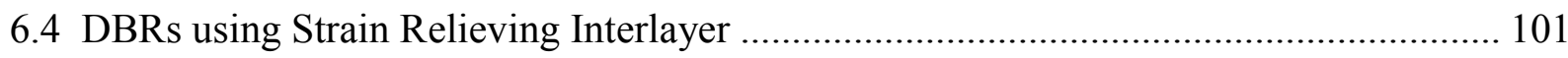

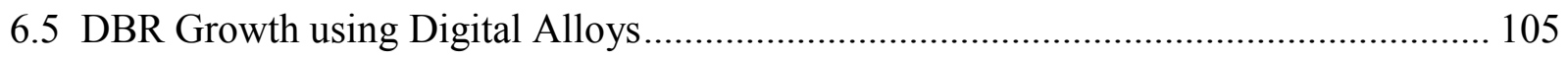

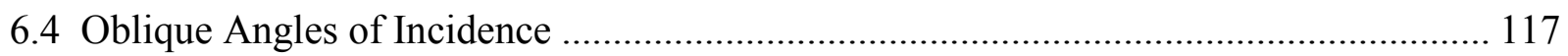

6.5 Influence of Strain on Refractive Index and DBR Reflectivity ..................................... 121

6.5.1 Influence of Strain on Reflectivity of Distributed Bragg Reflectors......................... 123

6.5.2 Measurement of Refractive Index .......................................................................... 126

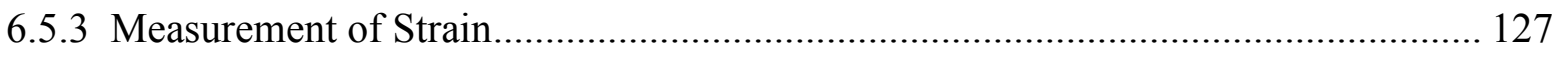

CHAPTER 6 - INTEGRATION OF DBRS WITH LIGHT EMITTING DIODES ................... 128

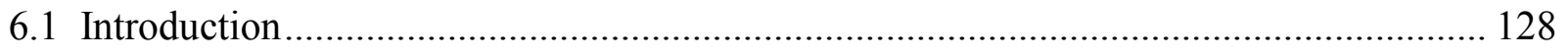

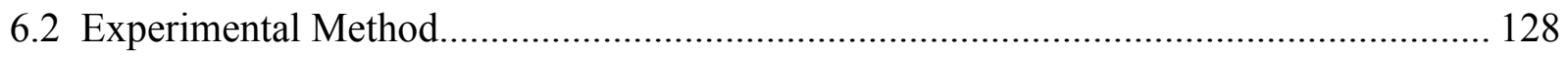

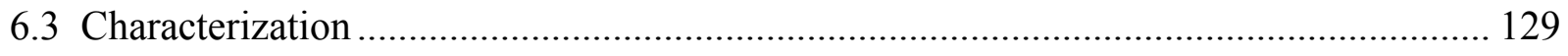

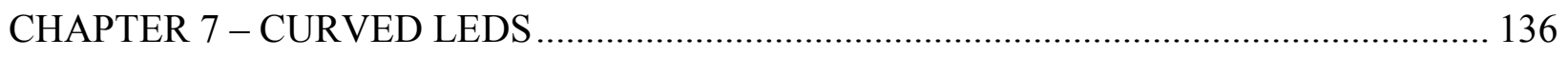

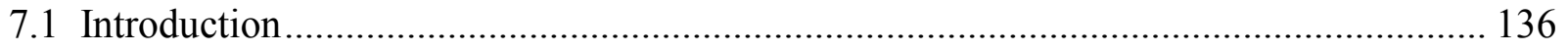

7.2 Experimental Methods ............................................................................................. 137

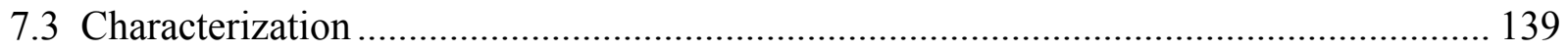


CHAPTER 8 -SELECTIVE AREA GROWTH AND EPITAIXIAL LATERAL

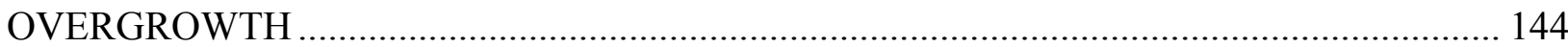

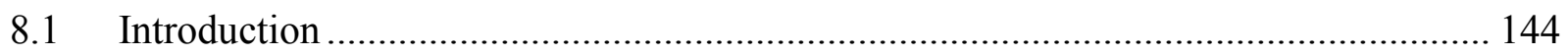

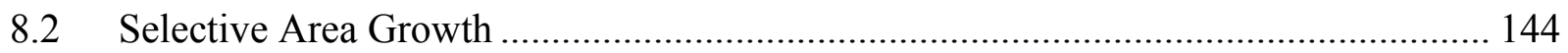

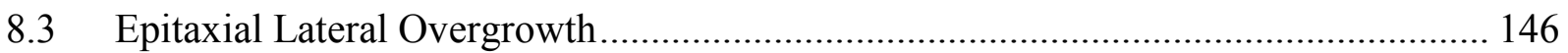

8.4 Selective Area Growth with No Rotation ................................................................. 148

CHAPTER 9 - ALUMINUM NITRIDE SUSPENDED STRUCTURES ................................. 154

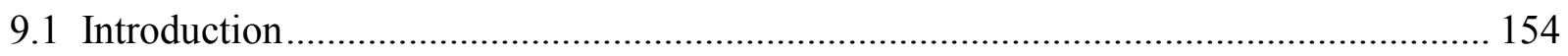

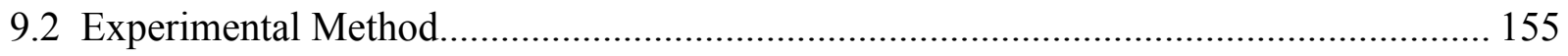

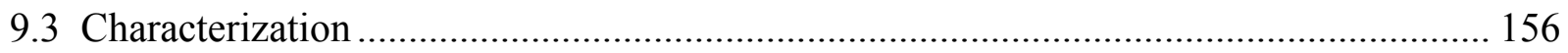

CHAPTER 10 - CONCLUSIONS AND FUTURE WORK ..................................................... 159

APPENDIX A: TEMPERATURE CALIBRATION........................................................... 162

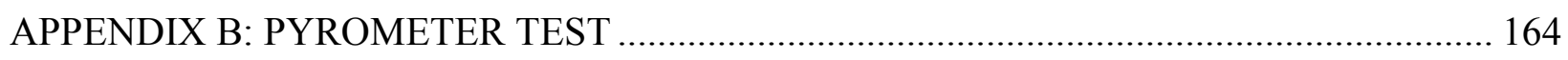

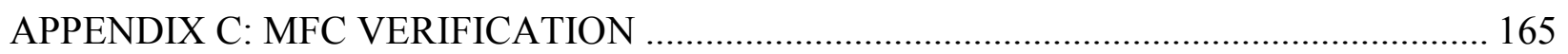

APPENDIX D: MATLAB CODE FOR CRACK ANALYSIS ................................................ 166

APPENDIX E: MATLAB CODE FOR DBR RESPONSE AT OBLIQUE ANGLES OF

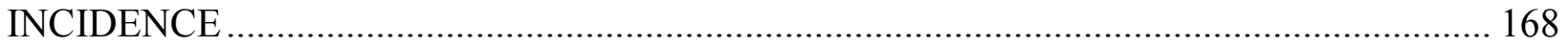

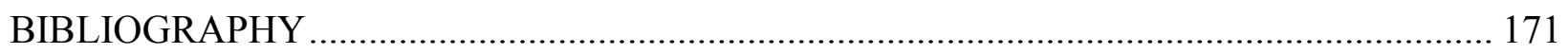




\section{I S T O F F I G U R E S}

Figure 1-1 - Generic schematic of III-Nitride based RCLED with integrated III-Nitride DBR.... 2

Figure 2-1 - a-lattice parameter verses bandgap for (Al,Ga,In)N hexagonal alloys. ...................... 6

Figure 2-2 - Wavelength of emission or detection of various semiconductor materials. Modified from Ref. 6. 6

Figure 2-3 - Wurtzite structure of GaN (modified from Ref. 2). Stacking sequence consists of two bilayers $\mathrm{A}$ and $\mathrm{B}$. 7

Figure 2-4 - Band gap energies $\operatorname{In}_{\mathrm{x}} \mathrm{Ga}_{1-\mathrm{x}} \mathrm{N}$ materials compared with the air-mass-1.5 solar

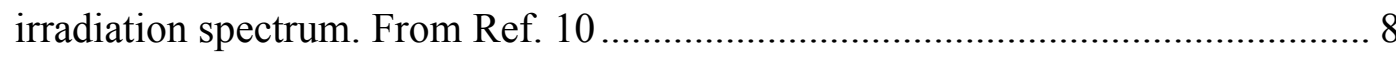

Figure 2-5 - Schematic of $\mathrm{Al}_{\mathrm{x}} \mathrm{Ga}_{1-\mathrm{x}} \mathrm{N}$ /GaN HEMT. From Ref. 17............................................ 10

Figure 2-6 - Band diagram of Ga-polar $\mathrm{Al}_{\mathrm{x}} \mathrm{Ga}_{1-\mathrm{x}} \mathrm{N} / \mathrm{GaN}$ junction. From Ref. 17........................ 10

Figure 2-7 - Schematic of typical InGaN MQW LED. Modified from Ref. 24.......................... 12

Figure 2-8 - MOVPE Growth Process Diagram. ......................................................................... 14

Figure 2-9 - GaN growth on sapphire using AlN nucleation layer. From Ref. 2 ........................ 17

Figure 2-10 - TEM image of GaN film grown on sapphire. High densities of dislocations are formed close to the sapphire interface. From Ref. 29.......................................... 17

Figure 2-11 - (a) ELOG mask pattern and (b) schematic of One Step Elog Growth.................... 19

Figure 2-12 - Illustration of Two Step ELOG. a) Step 1 involves the formation of structures with triangular cross section. b) In Step 2, lateral growth is induced to achieve coalescence.

Figure 2-13 - Schematic of $\mathrm{Al}_{\mathrm{x}} \mathrm{Ga}_{1-\mathrm{x}} \mathrm{N}$ layers grown on sapphire (a) with and (b) without AlN interlayers. Modified from Ref. 48.

Figure 2-14 - Illustration of surfactant effects of indium during AIN growth. Indium floats on the surface to allow the $\mathrm{Al}$ species to diffuse to the energetically favorable location where it is incorporated into the lattice. 25

Figure 2-15 - (a) Surface roughness of GaN grown with varying amounts of indium. (b) $\Delta \mathrm{c} / \mathrm{c}_{\mathrm{o}}$ as a function of $\Delta \mathrm{a} / \mathrm{a}_{0}$. The dashed line denotes the relationship between $\Delta \mathrm{c} / \mathrm{c} 0$ and $\Delta \mathrm{a} / \mathrm{a} 0$ estimated by calculation. The direction of the arrows indicates increasing indium flow rate. From Ref 57,58. 26

Figure 2-16 - (a) AFM Image of AlN/GaN MQW(right) and Al(In)N/GaN MQW(left). (b) XRD rocking curve around $0^{\text {th }}$ order satellite peak for AlN/GaN MQW structureswith and without indium. From Ref. 60.

Figure 2-17 - Illustration of $\mathrm{Al}_{0.75} \mathrm{Ga}_{0.25} \mathrm{~N}$ digital alloy comprised of $1 \mathrm{ML}$ of $\mathrm{GaN}$ and $3 \mathrm{ML}$ of AlN. 28 
Figure 2-18 - Illustration of DBR. Layer 1 has refractive index $n_{1}$ and layer 2 has refractive

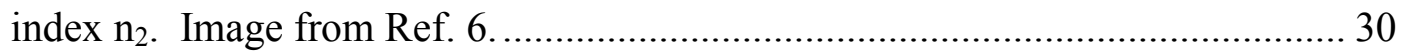

Figure 2-19 - Reflectivity of $\mathrm{Al}_{\mathrm{x}} \mathrm{Ga}_{1-\mathrm{x}} \mathrm{N} / \mathrm{GaN}$ DBR with increasing number of periods. From Ref. 75.

Figure 2-20 - (a) Reflectivity of 35 pair $\mathrm{Al}_{0.34} \mathrm{Ga}_{0.66} \mathrm{~N} / \mathrm{GaN}$ DBR (From Ref. 75) and (b) reflectivity of 20.5 Period AlN/GaN DBR (From Ref. 77). ............................. 32

Figure 2-21 - Lattice constant versus bandgap for (A1,Ga,In)N materials. From Ref. 79. ......... 34

Figure 2-22 - Refractive index contrast of AlInN alloys as a function of the indium concentration. From Ref. 79.

Figure 2-23 - Reflectivity spectra of (a) 20 and (b) 40 period $\mathrm{Al}_{\mathrm{x}} \mathrm{In}_{1-\mathrm{x}} \mathrm{N} / \mathrm{GaN} \mathrm{DBR}$. From Ref. 79,82 . 35

Figure 2-24 - Cross sectional SEM images, optical microscope images, and reflectivity measurements for 40 period $\mathrm{Al}_{0.35} \mathrm{Ga}_{0.65} \mathrm{~N} / \mathrm{GaN}$ DBRs. From Ref. 78. 36

Figure 2-25 - (a) Schematic and (b) reflectivity response of 20 pair AlN /GaN DBR with and without 5.5 periods of AlN/GaN superlattice insertion layers. From ref. 76....... 37

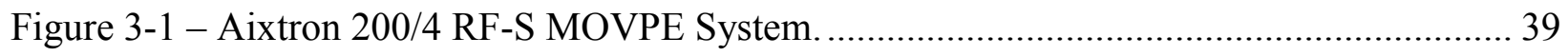

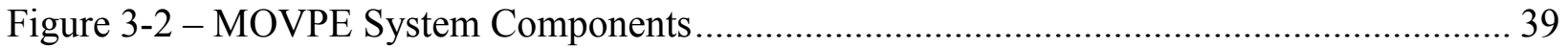

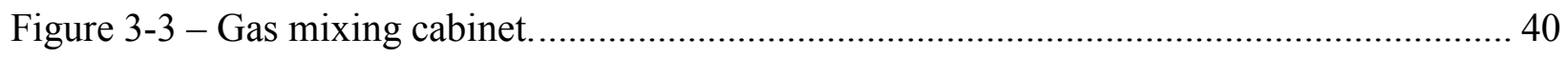

Figure 3-4 - Illustration of AIXTRON MOVPE system................................................ 41

Figure 3-5 - Measured substrate temperature and setpoint before and after calibration. Eurotherm reading corresponds to the setpoint entered in the CACE software. ... 42

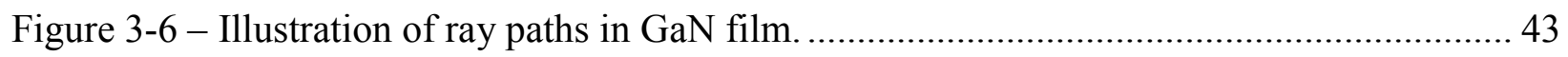

Figure 3-7 - EpiRas oscillations during GaN growth on sapphire. ....................................... 44

Figure 3-8 - Growth rate of GaN at different temperatures for various TMGa flow rates. ........ 45

Figure 3-9 - Growth rate of GaN under various TMGa flow rates. Ammonia source was fixed at $1500 \mathrm{sccm}$ and temperature was $990{ }^{\circ} \mathrm{C}$. 46

Figure 3-10 - Growth rate of GaN at different (a) ammonia source flows and (b) ammonia push flows. TMGa flow rate was $22.5 \mathrm{sccm}$ and the temperature was at $990{ }^{\circ} \mathrm{C}$. 47

Figure 3-11 - Relationship between the reactor pressure and $\mathrm{GaN}$ growth rate. Ammonia source was $1500 \mathrm{sccm}$, TMGa source was $22.5 \mathrm{sccm}$, and reactor temperature was $990{ }^{\circ} \mathrm{C}$. 48

Figure 3-12 - Reactor fill time for different MFCs. ........................................................... 49

Figure 3-13 - Illustration of X-Ray Diffraction. Modified from Ref. 87 ............................. 50 
Figure 3-14 - Schematic showing relationship between real lattice parameters and reciprocal lattice space. Modified from Ref. 87 .......................................................... 52

Figure 3-15 - Example reciprocal space mapping of a (a) strained and (b) relaxed $\mathrm{Al}_{\mathrm{x}} \mathrm{Ga}_{1-\mathrm{x}} \mathrm{N}$ layers on GaN. From Ref. 87. .................................................................. 52

Figure 3-16 - Transmission setup using white light source, CCD spectrometer, and fiber optic

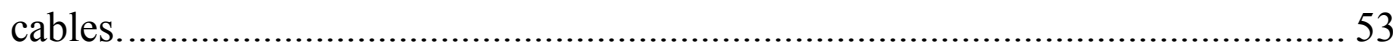

Figure 3-17 - Transmission of GaN film on sapphire .................................................. 54

Figure 3-18 - Plot of interference fringes in transmission of $\mathrm{GaN}$ on sapphire sample.............. 54

Figure 3-19 - Extraction of bandgap from transmission measuremetns. ............................... 56

Figure 3-20 - Setup for measurement the reflectivity of a sample at normal incidence............ 56

Figure 3-21 - Reflectivity of the aluminum coated pyrex mirror used in for reflectivity measurements in this work........................................................................ 58

Figure 3-22 - Reflectivity of an III-Nitride based DBR ..................................................... 58

Figure 3-23 - Ellipsometry measurement techinque process flow. From Ref. 91 ................... 59

Figure 3-24 - Schematic of Van Der Pauw contact geometry. Image from Ref. 93.................. 61

Figure 3-25 - Relationship between carrier mobility and bulk carrier concetration for n-type GaN.

Figure 3-26 - Bulk carrier concetration of n-type $\mathrm{GaN}$ as a function of the ratio of silane dopant to group III source supplied. .....

Figure 4-1 - (a) Schematic of $\mathrm{Al}_{\mathrm{x}} \mathrm{Ga}_{1-\mathrm{x}} \mathrm{N}$ digital alloy on sapphire substrate and (b) pulse flow scheme for growth. 66

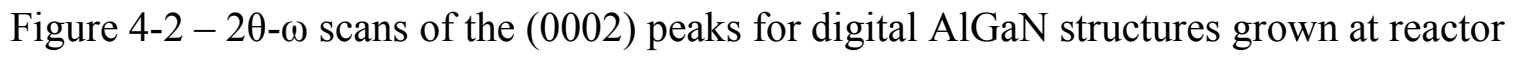
pressures of 200, 100, 65, and 50 mbar.

Figure 4-3 - Apparent lattice parameter of $\mathrm{Al}_{\mathrm{x}} \mathrm{Ga}_{1-\mathrm{x}} \mathrm{N}$ digital alloys calculated from the (0002), (0004), and (0006) reflections. 68

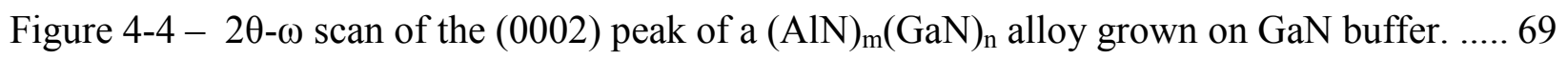

Figure 4-5 - Reciprocal space map of (1014) peak fortwo different $(\mathrm{AlN})_{\mathrm{m}}(\mathrm{GaN})_{\mathrm{n}}$ alloys grown on GaN buffer. (a) Shows a psuedocoherent film and (b) shows a relax film...... 70

Figure 4-6 - Period of the digital alloys as a function of AIN growth time for alloys grown on $\mathrm{GaN}$ and AlN buffer layers. 72

Figure 4-7 - AlN mole fraction of the digital $\mathrm{Al}_{\mathrm{x}} \mathrm{Ga}_{1-\mathrm{x}} \mathrm{N}$ film calculated from the periodicity... 73

Figure 4-8 - a- and c- lattice parameters for digital alloys grown on GaN buffer layers............ 74 Figure 4-9 - a- and c-lattice parameters for digital alloys grown on AlN buffer layers............. 75 
Figure 4-10 - Comparison of AIN mole fraction calculated from the periodicity and from Vegard's law.

Figure 4-11 - Comparison of AIN mole fraction calculated from the periodicity and EDS measurements. Plot includes digital alloys grown on GaN and AlN buffer layers and also random alloys grown on $\mathrm{GaN}$ buffer layers.

Figure 4-12 - In-plane strain in digital alloys with respect to the nominally relax lattice parameter. 78

Figure 4-13 - In-plane strain in digital alloys with respect to the underlying buffer layer. ........ 79

Figure 4-14 - RMS surface roughness for digital $\mathrm{Al}_{\mathrm{x}} \mathrm{Ga}_{1-\mathrm{x}} \mathrm{N}$ films. .................................... 80

Figure 4-15 - Comparison of strain in digital alloys and theoretical work. 81

Figure 4-16 - Comparison of strain in digital alloys and other experimental work on short period superlattices.

Figure 4-17 - Bandgap of digital alloy as extracted from transmission measurements as a function of the composition.

Figure 4-18 - Refractive index of digital alloys measured from ellipsometry.... 84

Figure 4-19 - Periodicity of digital alloys with varying GaN growth times. 84

Figure 4-20 - Growth rate of GaN layer in digital alloy as a function of the thickness of the GaN layer. As the thickness increases, the growth rate approaches the nominal value for thick $(>500 \mathrm{~nm})$ films. 85

Figure 4-21 - Periodicity of digital alloys grown with a GaN/AlN growth time a $1 \mathrm{~s} / 14 \mathrm{~s}$ and $1 \mathrm{~s} / 8 \mathrm{~s}$ at various temperatures. 86

Figure 5-1 - Oversimplified GaN based LED device showing possible light ray paths. Image modified from Ref. 104.

Figure 5-2 - Targeted structure in this work include the incorporation of III-Nitride based DBR under active region of LED................................................................... 88

Figure 5-3 - Schematic of AlN/GaN DBR structure. ...................................................... 90

Figure 5-4 - (a) Original microscope image of AlN/GaN DBR and (b) corresponding binary image. 91

Figure 5-5 - Cross Ssection SEM image of six period AlN/GaN DBR ................................. 93

Figure 5-6 - Reflectivity spectra of three different six period AlN/GaN DBR structures.......... 93

Figure 5-7 - Crack length per square millimeter as a function of the Trimethylindium supplied

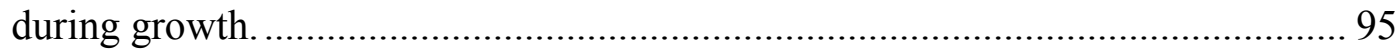

Figure 5-8 - SIMs mesurement of indium incorporation in AIN/GaN DBR. ......................... 96 
Figure 5-9 - (0002) reflection of the six period AlN/GaN DBR 97

Figure 5-10 - Difference between the AlN and GaN c-lattice parameter derived from the (0002) reflection as a function of the cracking in the films. 98

Figure 5-11 - Apparent GaN c-lattice parameter in the AlN/GaN DBR as a funciton of temperature

Figure 5-12 - Apparent AlN c-lattice parameter in the AlN/GaN DBR as a funciton of temperature

Figure 5-13 - Percent mismatch between the AlN and GaN lattice parameters in DBRs grown with no indium. 100

Figure 5-14 - Percent mismatch between the AlN and GaN lattice parameters in DBRs grown with a TMIn/TMAl ratio of 0.3 . 101

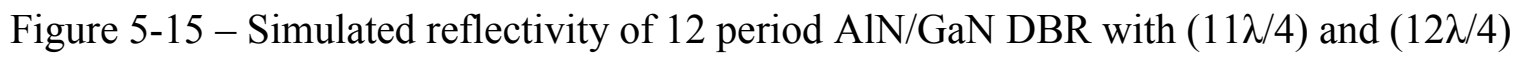
interlayer thicknesses.

Figure 5-16 - Cross sectional SEM image of 12 period AlN/GaN DBR with $11 \lambda / 4 \mathrm{GaN}$ interlayer.

Figure 5-17 - Measured reflectivity for 12 period AIN/GaN DBR with 11 $/ 4$ GaN interlayer. 103

Figure 5-18 - Measuremed reflectivity for an 18 period AlN/GaN DBR with $11 \lambda / 4 \mathrm{GaN}$ interlayer. 104

Figure 5-19 - AFM image of $600 \mathrm{~nm} \mathrm{nGaN}$ overgrowth on 12 period AlN/GaN DBR with interlayer. Z-scale is $30 \mathrm{~nm}$. 104

Figure 5-20 - Schematic of DBR containing $\mathrm{GaN}$ and digital $\mathrm{Al}_{\mathrm{x}} \mathrm{Ga}_{1-\mathrm{x}} \mathrm{N}$ layers. 105

Figure 5-21 - Cross sectional SEM image of a 12 period digital $\mathrm{Al}_{\mathrm{x}} \mathrm{Ga}_{1-\mathrm{x}} \mathrm{N} / \mathrm{GaN}$ DBR 107

Figure 5-22 - Cross sectional SEM image of 25 period digital $\mathrm{Al}_{\mathrm{x}} \mathrm{Ga}_{1-\mathrm{x}} \mathrm{N} / \mathrm{GaN}$ DBR in which the growth conditions have been modified for faster switching times. 108

Figure 5-23 - Reflecitivty of Digital $\mathrm{Al}_{\mathrm{x}} \mathrm{Ga}_{1-\mathrm{x}} \mathrm{N} / \mathrm{GaN}$ DBR as a function of the number of periods. 109

Figure 5-24 - Reflectivity of digital $\mathrm{Al}_{\mathrm{x}} \mathrm{Ga}_{1-\mathrm{x}} \mathrm{N} / \mathrm{GaN}$ DBRs with total periods ranging from 6 to 25 . 109

Figure 5-25 - Microscope images of the surface of a 25 period digital $\mathrm{Al}_{\mathrm{x}} \mathrm{Ga}_{1-\mathrm{x}} \mathrm{N} / \mathrm{GaN} \mathrm{DBR}$ and a 25 period AIN/GaN DBR. 110

Figure 5-26 - Reflectivity of 25 period digital $\mathrm{Al}_{\mathrm{x}} \mathrm{Ga}_{1-\mathrm{x}} \mathrm{N} / \mathrm{GaN} \mathrm{DBR}$ and 25 period $\mathrm{AlN} / \mathrm{GaN}$ DBR. 110

Figure 5-27 - (0002) reflection of 25 period digital $\mathrm{Al}_{\mathrm{x}} \mathrm{Ga}_{1-\mathrm{x}} \mathrm{N} / \mathrm{GaN} \mathrm{DBR}$ and 25 period AlN/GaN DBR. 
Figure 5-28 - Reciprocal space map of (-104)reflection for 25 period digital $\mathrm{Al}_{\mathrm{x}} \mathrm{Ga}_{1-\mathrm{x}} \mathrm{N} / \mathrm{GaN}$ DBR.

Figure 5-29 - Cracking in the 2" DBR from the center of the wafer to the edge....................... 113

Figure 5-30 - Reflectivity value at $452 \mathrm{~nm}$ mapped over a 2" DBR....................................... 114

Figure 5-31 - Reflectivy of 25 period DBR at different points along the 2" wafer. .................. 114

Figure 5-32 - Top Row: AFM images of GaN buffer layers used as substrates for DBR growth. Bottom Row: AFM images of $600 \mathrm{~nm}$ of $\mathrm{n}-\mathrm{GaN}$ grown on top of 20 period DBRs.

Figure 5-33 - Top Row: Reflectivity map at $452 \mathrm{~nm}$, Second Row: Reflectivity response at the center of the wafer, Bottom Row: Crack free area from the center for the 2" wafer.

Figure 5-34 - Schematic of 18 period AlN/GaN DBR used in the calculation for DBR response at oblique angles of incidence.

Figure 5-35 - Reflectivity of 18 period AIN/GaN DBR at oblique angles of incidence for (a)TE and (b) TM polarizations.

Figure 5-36 - Reflectivity at $460 \mathrm{~nm}$ for an 18 period AlN/GaN DBR for angles of incidence ranging from 0 to 80 degrees.

Figure 5-37 - Refractive index of AIN (left) and GaN (right) at $460 \mathrm{~nm}$ as a function of in-plane strain calculated from Eq. 5-18 using the theoretical and experimental values of elasto-optic coefficient shown in Table 5-1. Note: Approximated value of elastooptic coefficient for AlN was estimated to be $\mathrm{p}_{11}+\mathrm{p}_{12}=0.508$. 125

Figure 5-38 - Reflectivity spectra of 18 period AlN/GaN DBR. GaN refractive index was 2.44 and AIN refractive index was 2.1 and 2.156 for relaxed and strained simulations respectively. 126

Figure 6-1 - Schematic of the LED structure. 128

Figure 6-2 - Reflectivity of the DBR substrates integrated with LED. (a) Six period DBR and (b) 12 period DBR reflectivity spectra. 130

Figure 6-3 - Cross sectional SEM of LED on (a) 6 period DBR and (b) 12 period DBR. ........ 131

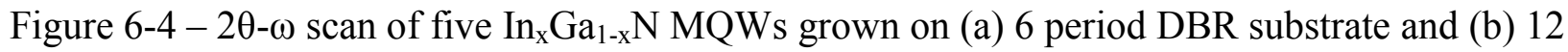
period DBR substrate.

Figure 6-5 - I-V characteristics for LED on DBR samples. 132

Figure 6-6 - (a) Optical Image of LED on 12 period DBRand (b) optical image of fabricated devices.

Figure 6-7 - EL spectra of LEDs with and without integrated DBRs. 134 
Figure 6-8 - Normalized LED intensity extracted from optical images.

Figure 7-1 - (a) Dielectric mask used for SAG. Stripes oriented along $<1 \overline{1} 00>$ direction. (b) SAG of GaN showing curved cross section. (c) LED growth on SAG of GaN. 138

Figure 7-2 - (a) Process flow for p-type contact deposition and mesa etch. (b) Process flow for n-

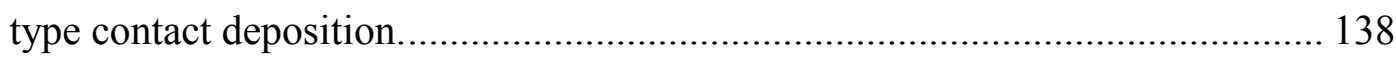

Figure 7-3 - Cross Sectional SEM of SAG of GaN using silicon nitride mask..................... 139

Figure 7-4 - (a) Fitting points along SAG structure as circle. (b) Relationship between the central angle of the fitted circle to the fill factor and V/III ratio.

Figure 7-5 - SEM image of fabricated LED on curved GaN SAG.

Figure 7-6 - (a) Current-Voltage characteristics of curved LED and (b) electroluminescence spectra of curved and planar LED................................................................ 143

Figure 8-1 - (a) Striped pattern along $\mathrm{GaN}<1100>$ direction 144

Figure 8-2 - Scanning Electron Microscopy images of GaN SAG as a function of temperature and V/III ratio. a.) Step-one at $960^{\circ} \mathrm{C}$ for 40 minutes, b.) Step-two at $1110^{\circ} \mathrm{C}$ for 90 minutes. c.) Step-one at $1050^{\circ} \mathrm{C}$ for 40 minutes, d.) Step-two ELOG at $1200^{\circ} \mathrm{C}$ for 90 minutes.

Figure 8-3 - Ratio of lateral to vertical growth rate as a function of growth temperature. 147

Figure 8-4 - Second step of ELOG at different growth temperatures. a) $1015^{\circ} \mathrm{C} \mathrm{b}$ ) $1040^{\circ} \mathrm{C}$ and c) $1065^{\circ} \mathrm{C}$

Figure 8-5 - AFM images of the surface morphology of two step ELOG process. 148

Figure 8-6 - (a) Striped pattern along GaN $<1 \overline{100}>$ direction (b) Samples oriented parallel and normal to the incoming gas flow during selective area growth of $\mathrm{GaN}$.

Figure 8-7 - SEM image of SAG base structure. Structure was identical for samples with stripes oriented normal or parallel to incoming gas flow.

Figure 8-8 - SEM images of selective area growth of GaN with $<1100>$ striped pattern oriented parallel and normal to the incoming gas flow. 151

Figure 8-9 - Cross section as a function of time for normal and parallel oriented samples. The lines are a linear fit of the data using the error bars as weight. The y-axis intercept corresponds to the cross sectional area of the base structure. 152

Figure 8-10 - Height as a function of growth time for parallel and normal oriented samples. The lines are a linear fit of the data using the error bars as weight. The y-axis intercept corresponds to the height of the base structure. 153

Figure 9-1 - Flow process for the fabrication of AlN suspended structures. 155 
Figure 9-2 - (a) AlN grown on $5 \mu \mathrm{m}$ wide $\mathrm{SiO}_{\mathrm{x}}$ stripe with $200 \mathrm{~nm}$ thickness (b) Free standing AlN micro-structures after removal of $\mathrm{SiO}_{x}$ sacrificial layer. Air gap height: 200nm, AlN thickness: 250nm, Width: $5 \mu \mathrm{m}$.

Figure 9-3 - (a) Array of $5 \mu \mathrm{m}$ wide AlN micro-structures. air gap height: $200 \mathrm{~nm}$ and AlN thickness: $250 \mathrm{~nm}$, (b) Closer view of corner of AlN micro-structure in (a), (c) AlN micro-structure, air gap height: 500nm, AlN thickness: 500nm, width: $24 \mu \mathrm{m}$. (d) AlN micro-structure, air gap height: $600 \mathrm{~nm}$, AlN thickness: 500nm, width: 90

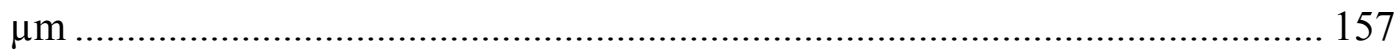




\section{I S T O F T A B L E S}

Table 2-1 - Description of crystal growth techniques.................................................... 13

Table 2-2 - Lattice parameters and thermal expansion coefficients of III-Nitride Alloys ${ }^{2,43, . . . . . ~} 22$

Table 4-1 - Lattice parameters and elastic coefficients for $\mathrm{GaN}^{43}$ and $\mathrm{AlN}^{2}$........................ 75

Table 5-1 - Constants for AlN and GaN including a and c-lattice parameters ${ }^{43}$, refractive

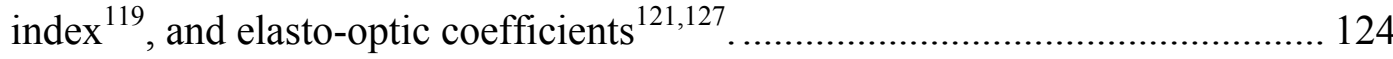




\section{IS T OF A CR ONYMS}

\begin{tabular}{|c|c|}
\hline 2D & : Two Dimensional \\
\hline $3 \mathrm{D}$ & : Three Dimensional \\
\hline AFM & : Atomic Force Microscopy \\
\hline AlN & : Aluminum Nitride \\
\hline $\mathrm{Al}_{x} \mathrm{Ga}_{1-x} \mathrm{~N}$ & : Aluminum Gallium Nitride \\
\hline BOE & : Buffered Oxide Etch \\
\hline $\mathrm{Cp}_{2} \mathrm{Mg}$ & : Bis-cyclopentadienyl Magnesium \\
\hline DBR & : Distributed Bragg Reflector \\
\hline DOE & : Department of Energy \\
\hline EDS & : Energy Dispersive X-Ray Spectroscopy \\
\hline EL & : Electroluminescence \\
\hline ELOG & : Epitaxial Lateral Overgrowth \\
\hline FWHM & : Full Width Half Maximum \\
\hline GaAs & : Gallium Arsenide \\
\hline GaN & : Gallium Nitride \\
\hline GMS & : Gas Mixing Cabinet \\
\hline HEMT & : High Electron Mobility Transistor \\
\hline HVPE & : Hydride Vapor Phase Epitaxy \\
\hline $\operatorname{In}_{x} \mathrm{Ga}_{1-x} \mathrm{~N}$ & : Indium Gallium Nitride \\
\hline IQE & : Internal Quantum Efficiency \\
\hline IR & : Infrared \\
\hline IV & : Current - Voltage \\
\hline LED & : Light Emitting Diode \\
\hline MBE & : Molecular Beam Epitaxy \\
\hline
\end{tabular}




$\begin{array}{ll}\text { MEMs } & : \text { Micro-electro-mechanical Systems } \\ \text { MFC } & : \text { Mass Flow Controller } \\ \text { MO } & : \text { Metal Organic } \\ \text { MOVPE } & : \text { Metal Organic Vapor Phase Epitaxy } \\ \text { MQW } & : \text { Multi-Quantum Well } \\ \text { NEMs } & : \text { Nano-electro-mechanical Systems } \\ \text { NH}_{3} & : \text { Ammonia } \\ \text { PC } & : \text { Pressure Controller } \\ \text { PECVD } & : \text { Plasma Enhanced Chemical Vapor Deposition } \\ \text { RCLED } & : \text { Resonant Cavity Light Emitting Diode } \\ \text { RF } & : \text { Radio Frequency } \\ \text { RMS } & : \text { Root Mean Square } \\ \text { SAG } & : \text { Selective Area Growth } \\ \text { SCCM } & : \text { Standard Cubic Centimeters per Minute } \\ \text { SEM } & : \text { Scanning Electron Microscopy } \\ \text { TEC } & : \text { Thermal Expansion Coefficient } \\ \text { TEGa } & : \text { Triethylgallium } \\ \text { TMAl } & : \text { Trimethylaluminum } \\ \text { TMIn } & : \text { Trimethylindium } \\ \text { TMGa } & : \text { Trimethylgallium } \\ \text { UV } & : \text { Ultra Violet } \\ \text { XRD } & : \text { X-Ray Diffraction } \\ & \end{array}$




\section{I S T O F S Y M B O L S}

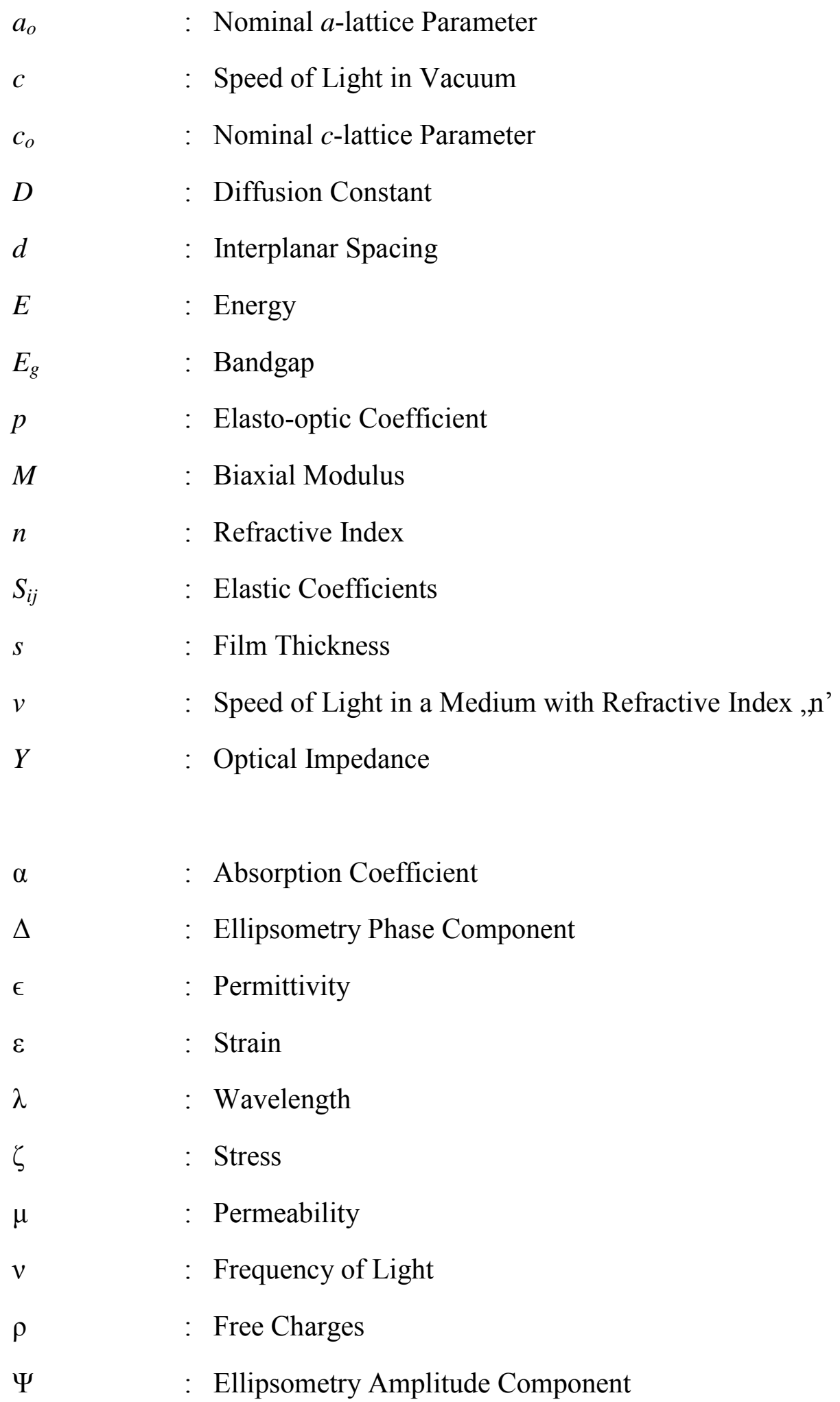




\section{H A P T ER $1-$ I N T R O D U C T I O N}

\subsection{Statement of the Problem}

The III-Nitride material system is attractive for a number of optoelectronic and electronic applications including but not limited to, Light Emitting Diodes (LEDs), Laser Diodes (LDs), solar cells, and High Electron Mobility Transistors (HEMTs) ${ }^{1}$. Although it has been studied for the last few decades, several issues ranging from material growth to device fabrication remain unresolved. One such issue includes strain management in III-Nitride heterostructures. The binary alloys in the $(\mathrm{Al}, \mathrm{In}, \mathrm{Ga}) \mathrm{N}$ family are characterized by a large difference in the lattice parameters $^{2}$ which leads to defect formation and cracking within heterostructures ${ }^{3}$. This degradation in material quality is detrimental to device fabrication. This work investigates growth based techniques to manage strain in III-Nitride heterostructures and thereby reduce the defect formation. Specifically, this work develops strain relieving techniques for the growth of high Aluminum (Al) content Aluminum Gallium Nitride $\left(\mathrm{Al}_{x} \mathrm{Ga}_{1-x} \mathrm{~N}\right)$ alloys and Aluminum Nitride (AIN) layers for use in III-Nitride based Distributed Bragg Reflectors (DBRs). Two different strain relieving techniques have been investigated to fabricate high quality, high reflectivity III-Nitride based Distributed Bragg Reflectors for use in visible wavelength optoelectronic device fabrication, such as Light Emitting Diodes (LEDs) or Resonant Cavity LEDs (RCLEDs). A generic structure of a RCLED structure utilizing a III-Nitride DBR is shown in Figure 1-1. The specific means of strain relief targeted in this work include the following:

- The use of indium as a surfactant during Metal Organic Vapor Phase Epitaxy

- The use of a digital alloy growth technique for high aluminum content Aluminum Gallium Nitride alloys

These techniques have been optimized in order to fabricate III-Nitride Based Distributed Bragg Reflectors (DBRs) with a reflectivity greater than 94\%. The DBRs have been integrated with visible wavelength LEDs to improve light extraction.

Other defects, such as threading dislocations ${ }^{1}$ also continue to plague the efficiency of III-Nitride based devices by forming non-radiative recombination and scattering centers ${ }^{1}$. 


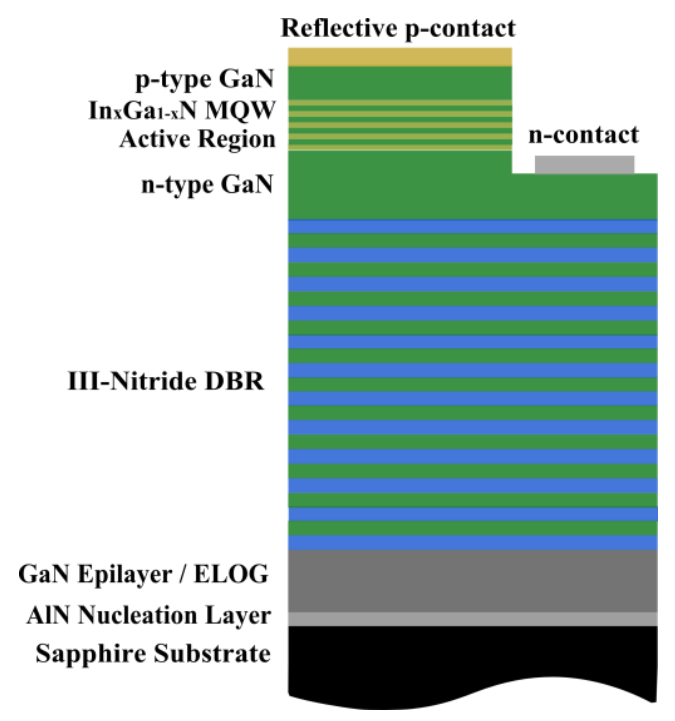

Figure 1-1-Generic schematic of III-Nitride based RCLED with integrated III-Nitride DBR.

Additionally, the piezoelectric and spontaneous polarization ${ }^{4}$ in the III-Nitride materials also plagues the operation of LEDs by causing carrier separation. Therefore, in addition to DBR fabrication, this work has also developed the Epitaxial Lateral Overgrowth (ELOG) process and three-dimensional structures using Selective Area Growth (SAG) techniques. The ELOG process reduces the dislocation density in order to improve the LED efficiency and was achieved using a standard two step process. The SAG of GaN has been used to create faceted structures consisting of semipolar and nonpolar planes. Although it is beyond the scope of this work, the SAG structures allow for the engineering of the polarization effects by fabricating devices on non-polar or semi-polar planes.

\subsection{Outline}

This work addresses both growth based approaches for minimizing strain in III-Nitride heterostructures. Chapter 2 covers the background related to this work. In particular, the properties of III-Nitride materials and also conventional growth techniques are discussed. As this work focuses on growth via MOVPE, this technique is discussed in detail. A relevant literature review of using indium as a surfactant and also digital alloy techniques is included. Also within Chapter 2 are reviews of Distributed Bragg Reflector (DBR) operation and a growth techniques used within the III-Nitride material system. 
Chapter 3 contains a summary of the experimental methods used in this work. In particular, a description of the Aixtron MOVPE tool used for material growth is provided. Diagnostic and maintenance procedures developed during the duration of this work are discussed in detail. In order to analyze and extract critical material properties from thin films such as composition, refractive index, and bandgap, many ex situ characterization techniques have been performed. In particular, films have been characterized via XRD, transmission, reflectivity, ellipsometry, and Hall Effect measurements. The system description and measurement technique for each is covered in Chapter 3.

Chapter 4 includes a description of the digital alloy growth technique employed in this work. This growth conditions used and the characterization of $\mathrm{Al}_{x} \mathrm{Ga}_{1-x} \mathrm{~N}$ alloys with an AlN mole fraction ranging from approximately 0 to 1 is discussed. The characterization of strain in these films with respect to the nominal lattice parameter and also the underlying buffer layer is also covered. Finally in this chapter, the influence of temperature on the growth kinetics is presented.

Chapter 5 describes the DBR growth and characterization using both indium as a surfactant and also digital $\mathrm{Al}_{x} \mathrm{Ga}_{1-x} \mathrm{~N}$ alloys. The growth technique as well as sample characterization including reflectivity, SEM, AFM, and XRD is included. Furthermore, an analysis of the cracking as observed through optical microscope images is discussed.

The integration of the DBRs with visible wavelength LEDs is covered in Chapter 6. In particular, blue $\operatorname{In}_{x} \mathrm{Ga}_{1-x} \mathrm{~N} / \mathrm{GaN}$ based LEDs have been grown on top of high reflectivity DBRs. The analysis of their performance as compared to conventional LEDs on GaN epilayers will be discussed. Characterization includes current-voltage measurements, electro-luminescence, and XRD.

In addition, LEDs have also been integrated on curved GaN surfaces and will be presented in Chapter 7. Using the SAG techniques, curved $\mathrm{GaN}$ features have been grown and used as the substrate for LED fabrication. The process flow as well as the LED characterization is included. 
Chapter 8 details the SAG and ELOG processes for GaN. The influence of the V/III ratio, growth temperature, and gas flow on the SAG geometry is presented. Optimal conditions for ELOG have been identified and are discussed.

Chapter 9 includes the fabrication of Aluminum Nitride (AIN) suspended structures using a sacrificial layer. Using the growth of AIN on silicon dioxide layers, suspended structures with a wide range of dimensions have been fabricated. These structures have numerous applications in micro-channel and micro-bridge technology given the piezoelectric properties of AlN. The fabrication technique and characterization are presented.

Finally, Chapter 10 contains the conclusions and future direction for this work. 


\section{H A P T E R 2 - B A C K G R O U N D}

\subsection{III-Nitride Materials}

III-Nitride based semiconductors possess several properties making them attractive for use in a number of devices, including but not limited to emitters, detectors, and transistors ${ }^{1}$. In particular, the wide, direct bandgap of binary, ternary, and quaternary alloys in the (Al,Ga,In)N family can be engineered from approximately $0.7 \mathrm{eV}^{5}$ to $6.2 \mathrm{eV}^{2}$ depending on the composition as shown in Figure 2-1. This allows the emission wavelength to range over the entire visible spectrum and into the Ultra Violet (UV) regime. This is the only direct semiconductor family that can cover this range ${ }^{6}$ as illustrated in Figure 2-2. Alloys in the IIINitride material family are most thermodynamically stable in the wurtzite crystal structure ${ }^{2}$. As shown in Figure 2-3, this is a hexagonal close packed structure consisting of two bilayers, A and B, with stacking sequence ABABAB.... Accordingly, this crystal structure is characterized by two lattice parameters, $a$ and $c$, where the $a$-lattice parameter is the edge length of the basal hexagon and the $c$-lattice parameter is the height of the hexagonal unit cell. The cubic zincblend structure also exists and is commonly seen when III-Nitrides are epitaxially grown on cubic substrates such as silicon or Gallium Arsenide ${ }^{2}$. This family of materials is further characterized by mechanical and thermal stability along with chemical inertness enabling its use in harsh environments $^{1}$. The high electron saturation velocity and high break down fields are attractive for High Electron Mobility Transistors (HEMTs) ${ }^{1,2}$ while the large piezoelectric properties ${ }^{2}$ are well suited for Microelectromechanical (MEMs) and Nanoelectromechanical (NEMs) devices.

Due to the range of wavelengths covered by this material family, considerable efforts have focused on fabricating visible and UV devices with III-Nitride materials and also improving the efficiency of such devices for Solid State Lighting (SSL) applications ${ }^{1}$. Popular visible lighting applications include traffic lights, signs, automotive, mobile devices, and general white lighting, while UV LEDs are often of interest for applications such as sterilization and biological detection $^{1}$. LEDs offer a potential increase in efficiency when compared to current lighting technologies such as incandescent, fluorescent, and high intensity discharge lamps and are being pursed in the United States as an alternative lighting solution ${ }^{7}$. The common approaches for creating white light using LEDs includes color mixing and phosphors. In color mixing, emission 


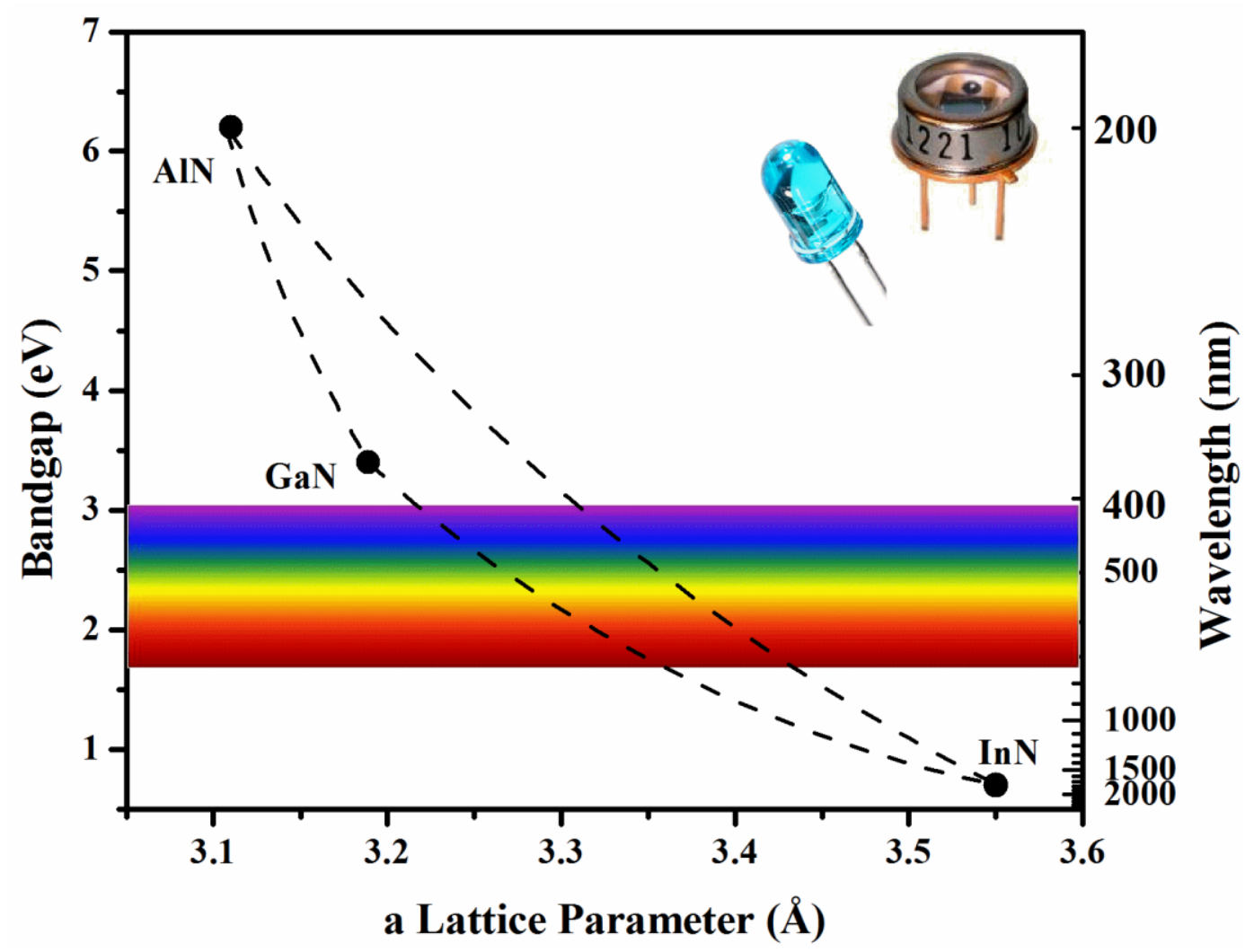

Figure 2-1 - a-lattice parameter verses bandgap for (Al,Ga,In)N hexagonal alloys.

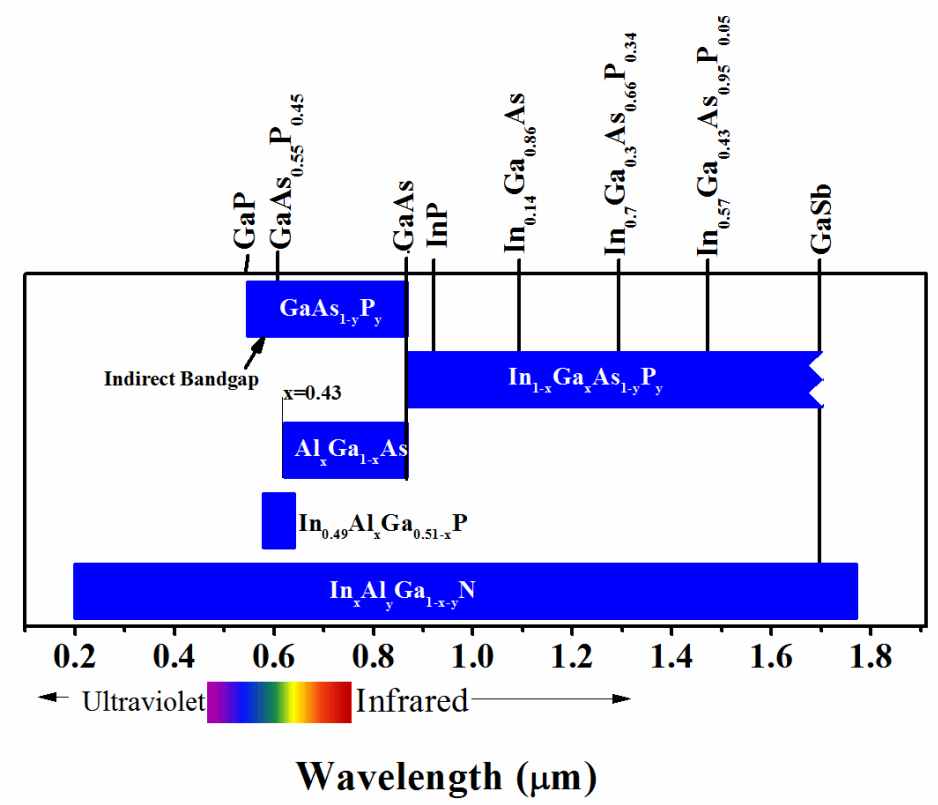

Figure 2-2 - Wavelength of emission or detection of various semiconductor materials. Modified from Ref. 6. 


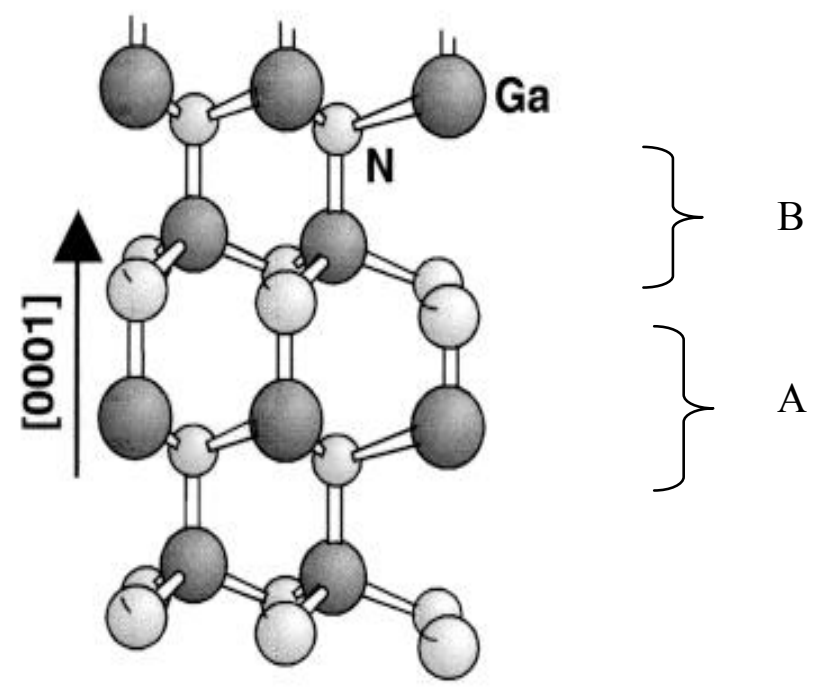

Figure 2-3 - Wurtzite structure of GaN (modified from Ref. 2). Stacking sequence consists of two bilayers $A$ and $B$.

from red, green, and blue emitters are combined to produce white light. Within the United States, the Department of Energy (DOE) has established a timeline for the development of SSL over the next 10-15 years ${ }^{7}$. Many of the problems that hinder the use of LEDs for general lighting applications include device efficiency and cost of the materials. Blue nitride based LEDs are commercially available and as of 2009 exhibited Internal Quantum Efficiencies (IQE) of around $80 \%$ however, the target established by DOE is $90 \%{ }^{7}$. Green nitride based LEDs present more of a challenge with typical IQEs around $50 \%$ in $2009^{7}$. As with the blue LEDs, the target for green emitting devices is $90 \%$. This lack of efficiency is due to several reasons. For visible emission, typically Indium Gallium Nitride $\left(\operatorname{In}_{x} \mathrm{Ga}_{1-x} \mathrm{~N}\right)$ Multi-Quantum Wells (MQWs) are employed as the active region in the LED devices as shown in the generic structure in Figure 1-1. Green emission requires larger indium concentrations in the $\operatorname{In}_{x} \mathrm{Ga}_{1-x} \mathrm{~N}$ wells when compared to blue devices as illustrated in Figure 2-1. Unfortunately, the growth of $\operatorname{In}_{x} \mathrm{Ga}_{1-x} \mathrm{~N}$ is challenging for a number of reasons. Phase separation occurs due to the difference in lattice constants between $\mathrm{InN}$ and $\mathrm{GaN}$, and the large immiscibility gap in this system ${ }^{8}$. This results in inhomogeneous In concentrations in planar films in the typical growth window of $600{ }^{\circ} \mathrm{C}$ to 800 ${ }^{\circ} \mathrm{C}^{9}$. Higher growth temperature can improve crystallinity, but results in a reduced In concentration. Furthermore, there is a large internal electric field occurring in nitride based devices grown along the $c$-axis due to the nonsymmetric nature of the wurtzite crystal ${ }^{2}$. These 
internal electric fields have detrimental effects on emitter devices as they cause carrier separation, reduced recombination, and a redshift in emission ${ }^{2}$. Additional work has focused on the investigation of $\operatorname{In}_{x} \mathrm{Ga}_{1-x} \mathrm{~N}$ growth on nonpolar and semipolar substrates which is of interest in order to reduce the internal electric field occurring in nitride based materials grown along the $c$ $\operatorname{axis}^{4}$.

In a similar fashion, $\mathrm{In}_{x} \mathrm{Ga}_{1-x} \mathrm{~N}$ MQWs are also attractive for solar cell applications ${ }^{10}$. As already discussed, bandgap of the $\operatorname{In}_{x} \mathrm{Ga}_{1-x} \mathrm{~N}$ material system covers the visible wavelengths; however it additionally corresponds closely with the solar radiation ${ }^{10}$ as shown in Figure 2-4. This makes the alloy a well suited material for photovoltaic applications. Furthermore, the IIINitride material system has been shown to exhibit a higher resistance to high-energy radiation damage. Space is one of the primary locations for solar cells however, the harsh environment often leads to the degradation of the optical and transport properties in materials such as Gallium Arsenide and Gallium Indium Phosphide ${ }^{10}$. This change has been attributed to defect states induced in the materials. III-Nitride based materials on the other hand, have been shown to be less sensitive to proton and electron bombardment making it a very attractive alternative material $^{10}$.

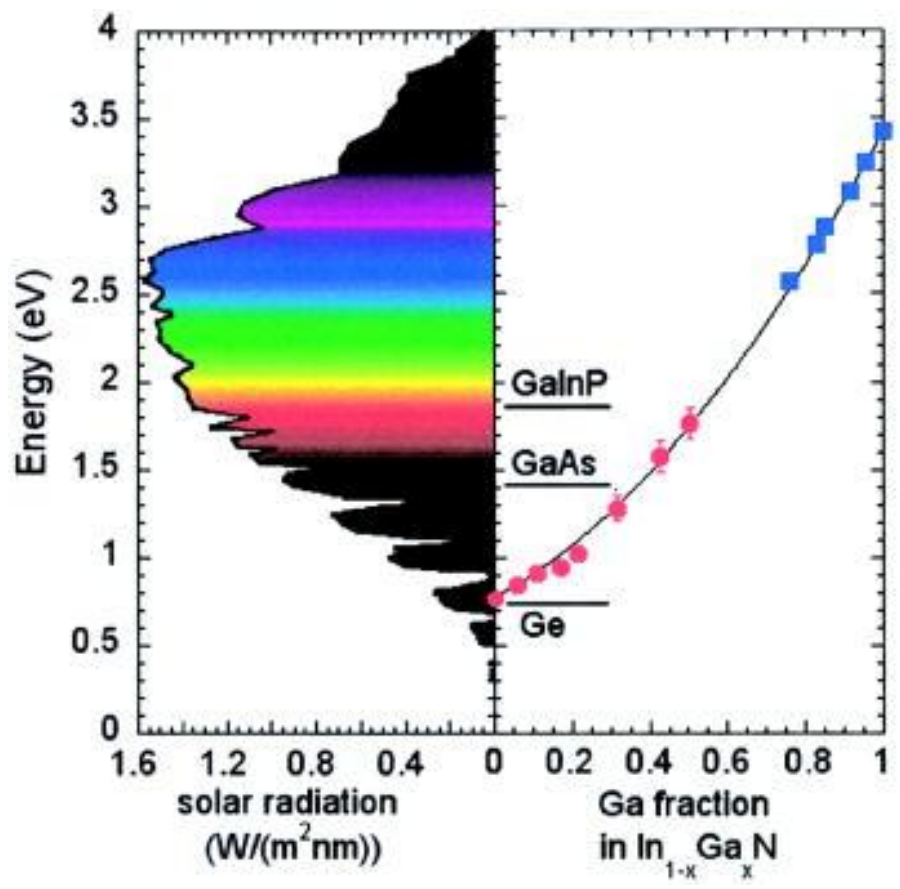

Figure 2-4 - Band gap energies $\operatorname{In}_{x} G a_{1-x} N$ materials compared with the air-mass-1.5 solar irradiation spectrum. From Ref. 10 
As shown in Figure 2-1, Aluminum Gallium Nitride $\left(\mathrm{Al}_{x} \mathrm{Ga}_{1-x} \mathrm{~N}\right)$ alloys within the IIINitride family are employed for UV emitting and detecting devices. Solid state emitters offer efficient and portable alternatives to large, toxic gas lasers and as a result are being heavily pursued for medical, sensor, and technological applications ${ }^{11}$. Medical applications largely involve sterilization of air and water, but also include various forms of fluorescence endoscopy for cancer diagnosis ${ }^{12}$. Solid state devices provide a compact, rugged, and portable device specifically for such applications. Optical UV biosensors are being pursued for label free detection of molecules ${ }^{13,14}$ and when compared to other types of detection, are inherently simpler and allow for field testing. Finally, UV sources allow for high density optical storage, smaller resolution photolithography processes, and line-of-sight short range communications ${ }^{11,15}$. $\mathrm{Al}_{x} \mathrm{Ga}_{1-x} \mathrm{~N}$ alloys are heavily pursued for detector fabrication due to the fact that they enable solar blind detector fabrication ${ }^{16}$. Most UV photodetectors, such as silicon based devices, are sensitive to visible light as well as UV light. However, for certain applications, it is necessary to reject visible emission and focus only on UV wavelengths. Applications include missile tracking, furnace control, engine monitoring, and flame detection ${ }^{16}$.

In addition to optoelectronic properties, $\mathrm{Al}_{x} \mathrm{Ga}_{1-x} \mathrm{~N}$ alloys have other material properties well suited for transistor applications. As communication technology expands, radio frequency and microwave power amplifiers continue to attract more attention ${ }^{17} . \mathrm{Al}_{x} \mathrm{Ga}_{1-x} \mathrm{~N}$ based High Electron Mobility Transistors (HEMTs) are one of the competitive devices. GaN based devices typically exceeded other materials in terms of high power, voltage, frequency, and temperature operation $^{17}$. In particular, the high breakdown voltage of GaN permits this material to operate at voltages much larger than other semiconductor devices such as GaAs based transistors ${ }^{17,18}$. This facilitates the fabrication of smaller devices and eliminates the need for voltage converters in communication systems ${ }^{17}$. A general schematic for an $\mathrm{Al}_{x} \mathrm{Ga}_{1-x} \mathrm{~N} / \mathrm{GaN}$ HEMT is shown in Figure 2-5. This consists of an $\mathrm{Al}_{x} \mathrm{Ga}_{1-x} \mathrm{~N}$ layer on an insulating GaN layer. Typical $\mathrm{Al}$ concentrations are around $30 \%{ }^{17,18,19}$. Due to the large band offset between $\mathrm{Al}_{x} \mathrm{Ga}_{1-x} \mathrm{~N}$ and $\mathrm{GaN}$, a triangle quantum well is formed at the interface as shown in Figure 2-6. As the electrons diffuse from the large bandgap material to the smaller bandgap material they are trapped in the quantum well forming a two-dimensional electron gas. The confined electrons exhibit a higher mobility due to the fact that there is no scattering with ionized impurity atoms. Saturation velocities reach $3 \times 10^{7}$ $\mathrm{cm} / \mathrm{s}$ and mobilities reach around $1200 \mathrm{~cm}^{2} / \mathrm{Vs}^{18}$. Very high power densities from 


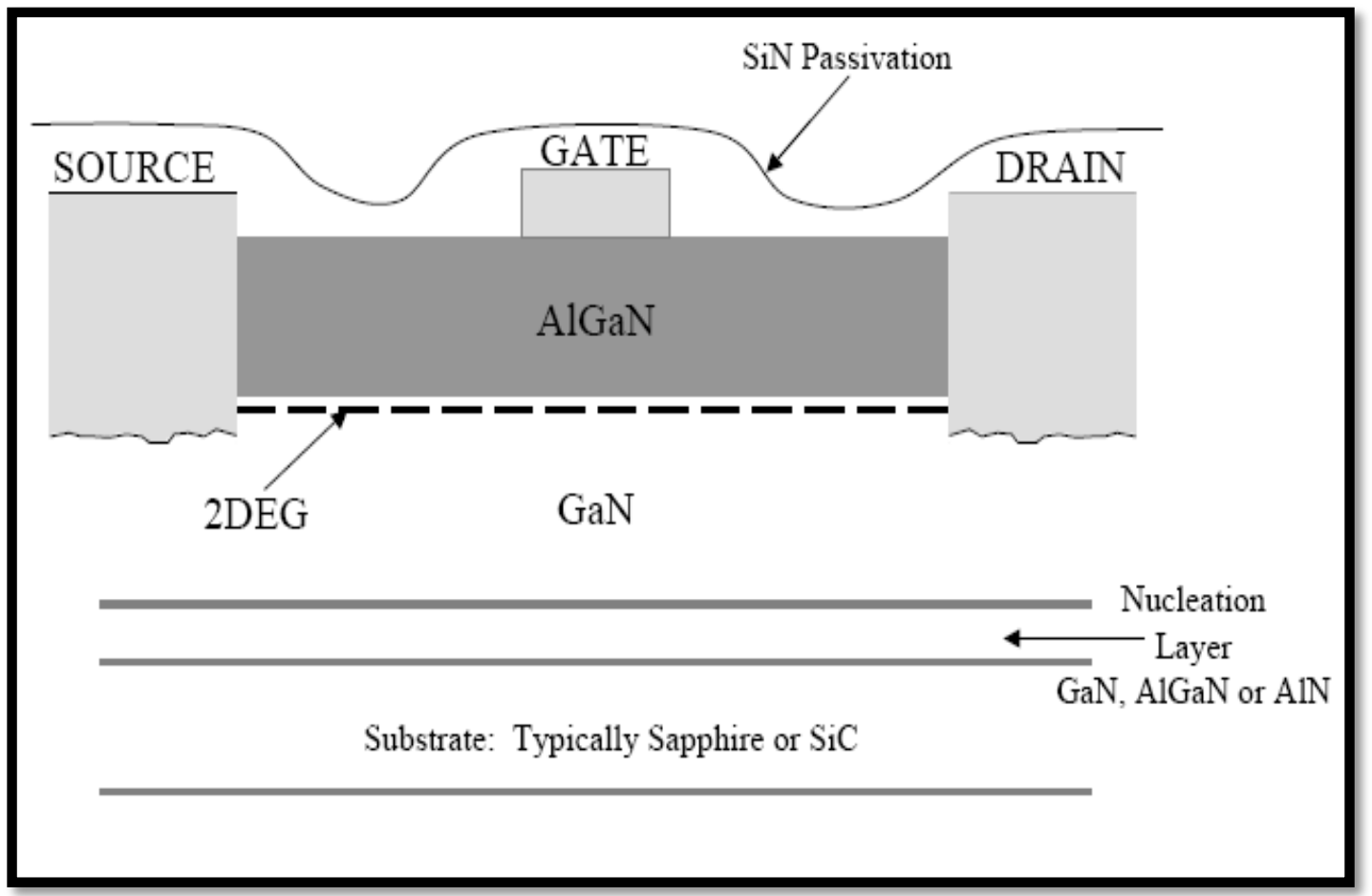

Figure 2-5 - Schematic of $A l_{x} G a_{1-x} N / G a N$ HEMT. From Ref. 17.

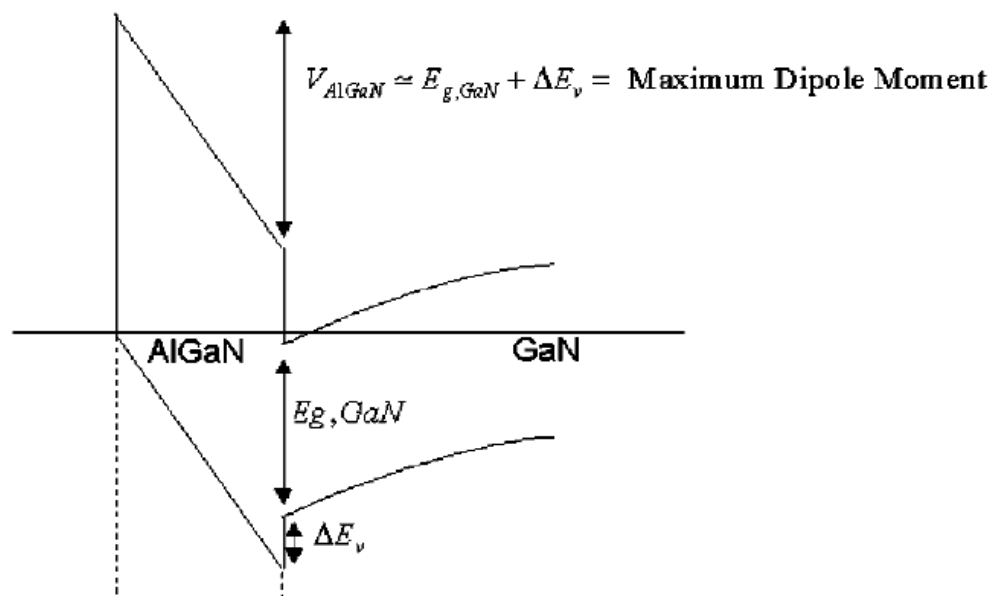

Figure 2-6-Band diagram of Ga-polar $A l_{x} G a_{1-x} N / G a N$ junction. From Ref. 17. 
$30-40 \mathrm{~W} / \mathrm{mm}$ have been reported ${ }^{20}$. Remaining issues largely include thermal management of these devices and also defects generated due to a lack of a native substrate. Furthermore, the operation at high voltages causes electric field induced degradation of the devices which remains an area of research interest ${ }^{18}$.

Gateless HEMT transistors are being pursued for biosensor applications due to the excellent bio-compatibility of the III-Nitrides ${ }^{21}$. By removing the metal gate shown in Figure 2-5 and attaching bio-agents, the surface potential is modified so that a measurable change in the drain to source current occurs.

Finally III-Nitride alloys exhibit piezoelectric properties suitable for MEMs and NEMs device fabrication ${ }^{22}$. MEMs typically refer to the fabrication of devices, such as sensors and actuators, which combine electrical and mechanical components on the micrometer or submicrometer size scale. Commercially available silicon and germanium devices include inkjet printer heads, accelerometers, pressure sensors, and flow sensors ${ }^{22}$. While silicon technology is well developed, there is still considerable interest in the use of III-Nitride layers to bring a new functionality to such devices. In particular, the chemical and thermal stability of the III-Nitride materials enables the use of MEMs devices in harsh environments ${ }^{22}$. Silicon for example, does not maintain it properties at high temperatures ${ }^{22}$. Furthermore, the bio-compatibility facilitates the fabrication of bio-sensors or bio-compatible devices such as micro-channels ${ }^{21}$. Using typical growth techniques as described in the later sections, atomic control over layers can be achieved within the III-Nitrides introducing an additional element of control in the design and fabrication of micro and nano-scale devices. Within the III-Nitride family, AlN exhibits the highest piezoelectric response and is widely pursed for MEMs and Surface Acoustic Wave devices ${ }^{2}$. Although the piezoelectric response is less than common piezoelectrics, such as Lead Zirconate Titanate (PZT) or Zinc Oxide ( $\mathrm{ZnO})$, the high thermal stability of $\mathrm{AlN}^{2,23}$ is attractive for specific applications.

All of the III-Nitride devices mentioned, including LEDs, photo-detectors, and HEMTs, consist of multi-layer heterostructures typically grown on foreign substrates as illustrated by the device schematic ${ }^{24}$ in Figure 2-7. Foreign substrates such as sapphire and silicon carbide are typically used due to the limited size and cost of bulk III-Nitride substrates. Bulk samples cannot be grown using conventional techniques, such as Czochralski or Bridgman methods due 


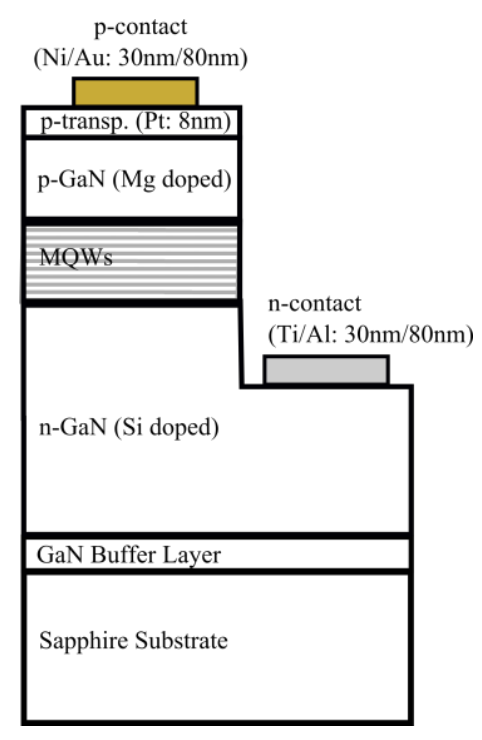

Figure 2-7-Schematic of typical InGaN MQW LED. Modified from Ref. 24.

to the high temperature and nitrogen pressure required to melt III-Nitride alloys (2497 K and 6 $\mathrm{GPa}$ respectively for $\mathrm{GaN})^{25}$. Several alternative techniques have been used to produce bulk GaN substrates including Hydride Vapor Phase Epitaxy (HVPE) and high pressure solution processes $^{25}$, however, GaN substrates remain expensive and small in size giving them limited use in device fabrication. Typical crystal growth techniques used for heteoepitaxy along with advantages and disadvantages of each are listed in Table 2-1. This work will utilize growth via Metal Organic Vapor Phase Epitaxy (MOVPE).

\subsection{Material Growth via Metal Organic Vapor Phase Epitaxy (MOVPE)}

MOVPE is a complex growth technique based on both gas phase and surface chemical reactions. In MOVPE, metalorganic and ammonia precursors are supplied in the gas phase to a heated growth site. Common metalorganic precursors, used as the group III supply during the growth of III-Nitrides include Trimethylgallium (TMGa), Trimethylaluminum (TMAl), and Trimethlyindium (TMIn). Ammonia $\left(\mathrm{NH}_{3}\right)$ is typically employed as the nitrogen source. The process relies on the decomposition of the metalorganic and ammonia sources in order to obtain the metal ( $\mathrm{Ga}, \mathrm{Al}$, or $\mathrm{In})$ and $\mathrm{N}$ species, respectively. The complete pyrolysis occurs mainly on the surface of the heated substrate, where the metal and nitrogen species 
Table 2-1 - Description of crystal growth techniques.

\begin{tabular}{|c|c|c|c|}
\hline $\begin{array}{l}\text { Growth } \\
\text { Technique }\end{array}$ & Description & Advantages & Disadvantages \\
\hline $\begin{array}{l}\text { Liquid Phase } \\
\text { Epitaxy(LPE) }\end{array}$ & Growth from melt & $\begin{array}{l}\text { Simple, high purity, } \\
\text { thick layers }\end{array}$ & $\begin{array}{l}\text { Poor thickness uniformity, } \\
\text { multilayer structures difficult, } \\
\text { limited flexibility in commercial } \\
\text { applications }\end{array}$ \\
\hline $\begin{array}{l}\text { Hydride Vapor } \\
\text { Phase Epitaxy } \\
\text { (HVPE) }\end{array}$ & $\begin{array}{l}\text { Reaction of hot metal } \\
\text { chlorides and ammonia. } \\
\text { Hot } \mathrm{HCl} \text { is passed over } \\
\text { group III elements to form } \\
\text { metal chlorides }\end{array}$ & $\begin{array}{l}\text { Fast growth rate, well } \\
\text { developed }\end{array}$ & $\begin{array}{l}\text { Al containing alloys difficult, } \\
\text { hazardous precursors, difficult to } \\
\text { control }\end{array}$ \\
\hline $\begin{array}{l}\text { Molecular Beam } \\
\text { Epitaxy(MBE) }\end{array}$ & $\begin{array}{l}\text { Elemental sources are } \\
\text { evaporated onto heated } \\
\text { substrate under UHV }\end{array}$ & $\begin{array}{l}\text { High purity, uniform } \\
\text { growth, abrupt } \\
\text { interfaces, in situ } \\
\text { monitoring }\end{array}$ & $\begin{array}{l}\text { Slow growth rates (Low } \\
\text { throughput), expensive operation } \\
\text { (time and supplies) }\end{array}$ \\
\hline $\begin{array}{l}\text { Metal Organic } \\
\text { Vapor Phase } \\
\text { Epitaxy (MOVPE) }\end{array}$ & $\begin{array}{l}\text { Gaseous precursors are } \\
\text { supplied to heated growth } \\
\text { site where pyrolysis occurs }\end{array}$ & $\begin{array}{l}\text { Flexible, abrupt } \\
\text { interfaces, high purity, } \\
\text { large area growth, } \\
\text { selective area growth, } \\
\text { in situ monitoring }\end{array}$ & $\begin{array}{l}\text { Most parameters to control during } \\
\text { growth, hazardous precursors, } \\
\text { expensive reactants }\end{array}$ \\
\hline
\end{tabular}

undergo a chemical reaction and the nitride film is deposited. Using $\mathrm{GaN}$ as an example, the basic reaction between TMGa $\left(\mathrm{Ga}\left(\mathrm{CH}_{3}\right)_{3}\right)$ and $\mathrm{NH}_{3}$ is the following:

$$
\mathrm{Ga}\left(\mathrm{CH}_{3}\right)_{3}+\mathrm{NH}_{3} \rightarrow \mathrm{GaN}+3 \mathrm{CH}_{4}
$$

Methane, $\mathrm{CH}_{4}$, is a waste by-product and is exhausted from the system. Figure 2-8 illustrates the growth process for GaN. Initially, the precursors enter the growth chamber and are transported through the reactor by forced convection, or flow due to an imposed pressure gradient. For a horizontal reactor design, a region of decreased gas velocity is formed close to any surface in the growth chamber that is parallel to the gas flow. This region of decreased velocity is referred to as the boundary layer. It is commonly approximated that mass transport through the boundary layer occurs via diffusion. While this is not entirely accurate, it is a simplistic model which yields an accurate description of the process. In the real MOVPE process, the hydrodynamic description is complicated by buoyancy forces and natural convection due to the temperature gradient between the heated growth site and cold walls of the growth chamber. These effects are 


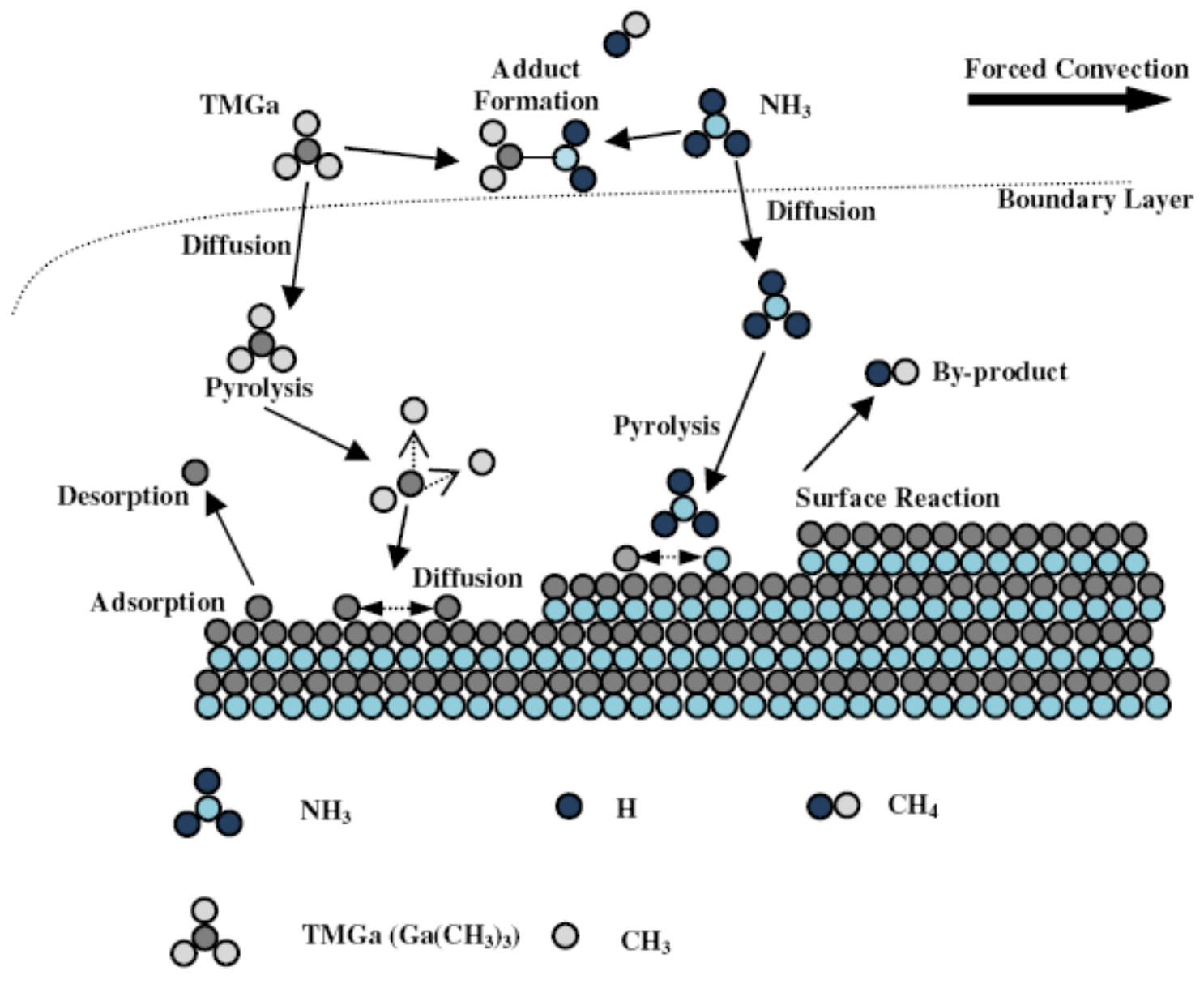

Figure 2-8-MOVPE Growth Process Diagram.

highly dependent on reactor geometry and must be modeled accordingly ${ }^{26}$. Upon reaching the heated growth site, the precursors undergo thermal decomposition. Although ammonia is very commonly used in MOVPE growth, it is not an ideal source due to its high thermal stability. Approximately $15 \%$ of ammonia decomposes at $\mathrm{GaN}$ growth temperatures of $950{ }^{\circ} \mathrm{C}^{26}$ requiring an excess of $\mathrm{NH}_{3}$ to be supplied during growth. Conversely, TMGa is almost completely dissociated at $500{ }^{\circ} \mathrm{C}^{26}$. The species decomposing before reaching the substrate continue to diffuse to the substrate due to the concentration gradients. Other species may not fully dissociate until reaching the heated substrate surface. Assuming the temperature is sufficient, the species diffuse on the substrate surface to an energetically favorable location at which point they are incorporated into the GaN film via a surface chemical reaction. Under ideal conditions, the 
growth surface is comprised of atomically flat terraces separated by steps as shown in Figure 2-8. Growth is modeled and has been observed in many crystals to occur by the propagation of these steps $^{26}$.

In general, growth can be categorized into two cases, kinetically limited and mass transport limited. Over a certain temperature range, growth is independent of temperature and is referred to as the mass transport limited regime ${ }^{26}$. The limiting factor in this case is gas phase diffusion of the precursors to the growth site. Under these conditions, the growth rate has been empirically shown to vary linearly with group III supply. As mentioned previously, the amount of ammonia supplied during growth is much greater than the amount of TMGa, giving rise to a $\mathrm{V} / \mathrm{III}$ ratio $>>1$, where the $\mathrm{V} / \mathrm{III}$ ratio is the ratio of $\mathrm{NH}_{3}$ to TMGa supplied. It is therefore assumed that that at the growth interface, there are considerably more $\mathrm{NH}_{3}$ molecules than TMGa molecules. Since $\mathrm{GaN}$ is stoichiometric and the same number of $\mathrm{Ga}$ and $\mathrm{N}$ atoms are incorporated into the solid, the growth rate of the film is therefore proportional to the concentration of the Ga atoms reaching the surface. Accordingly, the growth rate can be approximately by the diffusion of Ga atoms through the boundary layer ${ }^{26}$ :

$$
\frac{d s}{d t}=D \frac{d p_{G a}}{d x}
$$

In this equation $s$ is the film thickness, $t$ is the time, $D$ is a diffusion constant of Ga through the boundary layer dependent on reactor conditions such as temperature and pressure, $p_{G a}$ is the concentration of $\mathrm{Ga}$, and $x$ is the distance traveled through the boundary layer. Typically, it is desirable to conduct growth in the mass transport limited regime in order to obtain the highest growth rate efficiency (i.e. ratio of growth rate to the input molar flow). In the kinetically limited case, the growth rate is dependent on the temperature due to the influence of temperature on the pyrolysis of the precursors, diffusion of the adatoms on the surface, and also the desorption of the precursors from the surface ${ }^{26}$.

\subsection{III-Nitride Growth}

\subsubsection{Gallium Nitride Growth}

During MOVPE growth, GaN does not wet sapphire well and therefore direct growth on sapphire results in three dimension growth ${ }^{2}$ yielding material that is not suitable for device 
fabrication. The use of buffer and nucleation layers have been employed in order to achieve high quality films with smooth morphology. In particular, either low temperature AlN buffer layers or low temperature GaN nucleation layers have been used successfully on (0001) c-plane sapphire for subsequent $\mathrm{GaN}$ growth ${ }^{1,2,27}$. In both cases, the purpose of the layer is to provide nucleation centers for the high temperature GaN growth.

The growth process for GaN on sapphire using an AlN buffer layer is shown in Figure 2-9. Low temperature AlN layers, deposited around $600{ }^{\circ} \mathrm{C}$, are mainly amorphous layers. This layer is partially crystallized as the temperature is increased to a $\mathrm{GaN}$ growth temperature around 950-1000 ${ }^{\circ} \mathrm{C}$ and forms a columnar structure on which the high temperature GaN nucleates. Initially, the GaN also grows in a columnar structure similar to that of the AlN. However, the $\mathrm{GaN}$ crystals well oriented with the substrate (along the $c$-axis) exhibit a faster growth rate as compared to the mis-oriented crystals and form $\mathrm{GaN}$ islands. These islands laterally expand to coalesce and form a smooth $\mathrm{GaN}$ layer ${ }^{28}$.

Growth using a GaN nucleation center is similar to that with an AlN layer. Growth begins with the low temperature $\mathrm{GaN}$ deposition resulting in small $\mathrm{GaN}$ islands with columnar structure. Next high temperature annealing and growth are performed in which these islands laterally expand and coalesce to also form a smooth layer ${ }^{2}$.

In either case, a large number of dislocations, or line defects in the crystal, are generated at the interface between sapphire and GaN. As growth proceeds, there is a reduction in dislocation density due to the formation of loops and partial dislocations. After approximately $300 \mathrm{~nm}$, the remaining dislocations are vertically threading in the $<0001>$ direction and continue to propagate throughout the film growth ${ }^{2}$ as shown in the Transmission Electron Microscopy (TEM) image ${ }^{29}$ in Figure 2-10. These dislocations are attributed by some to accommodate the twist and tilt in the coalescing islands ${ }^{30}$ or from faulted regions in the nucleation layer ${ }^{28,31}$. Despite the generation mechanism, threading dislocations are by far the most predominant defect in GaN with densities ranging from $10^{9}$ to $10^{11} \mathrm{~cm}^{-2}$ for MOVPE grown films on sapphire substrates $^{1}$. After the initial filtering of the dislocations close to the substrate interface, three vertically threading dislocations are observed in high quality GaN layers: edge, screw, and mixed dislocations $\mathrm{s}^{1,30,31}$. Of typical defect densities, approximately $1 \%$ are screw dislocations with a 


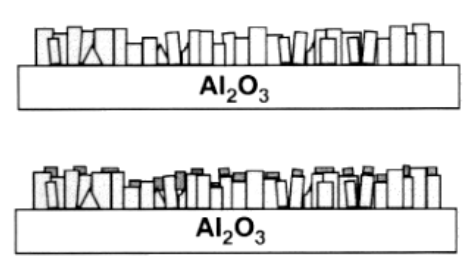

AIN buffer or nucleation layer $\left(\mathrm{T}_{\mathrm{S}}=600-700^{\circ} \mathrm{C}\right.$, $\mathrm{d}(\mathrm{AIN})=\mathbf{2 5 - 8 0} \mathrm{nm})$
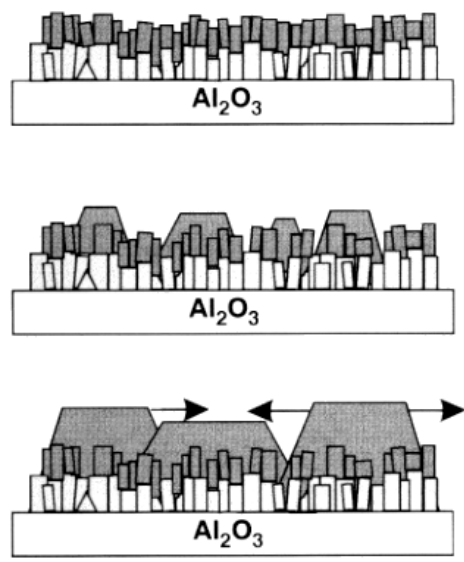

$(d(G a N)=200-300 \mathrm{~nm})$

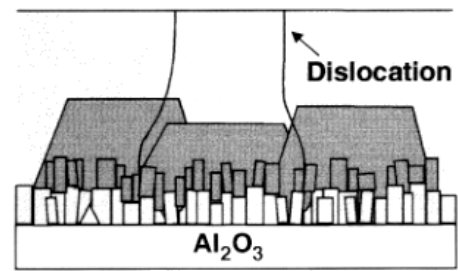

geometric selection $(\mathrm{d}(\mathrm{GaN})=50 \mathrm{~nm})$

island growth of GaN

lateral growth

nucleation of GaN

$\left(T_{S}=950-1050^{\circ} \mathrm{C}\right)$

2D-growth

$(\mathrm{d}(\mathrm{GaN})>300 \mathrm{~nm})$

Figure 2-9-GaN growth on sapphire using AlN nucleation layer. From Ref. 2.

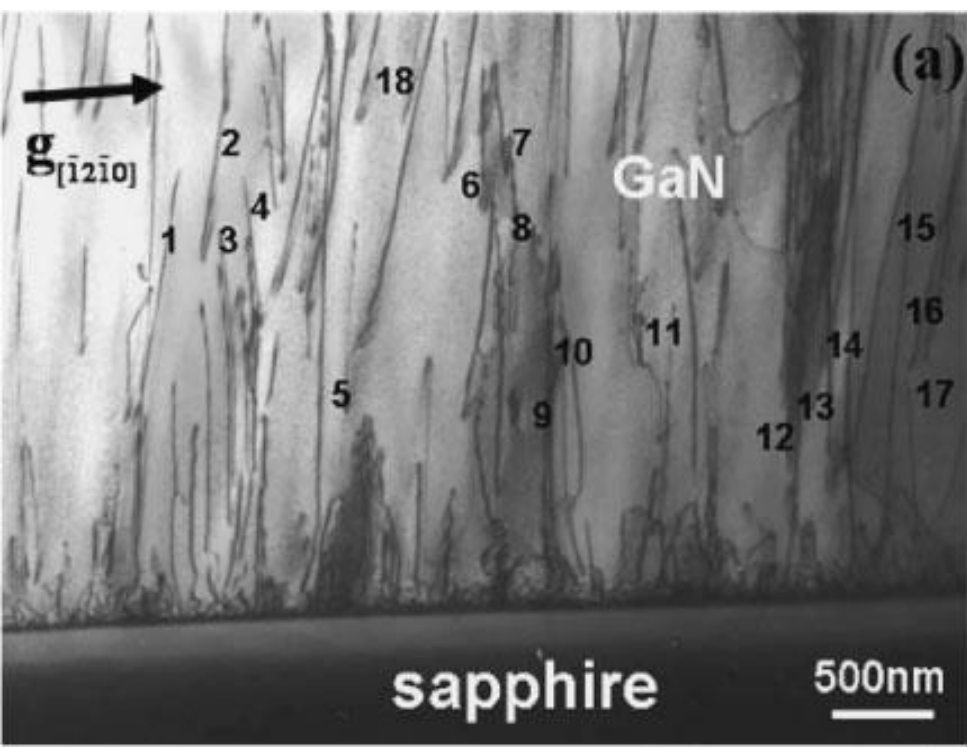

Figure 2-10 - TEM image of GaN film grown on sapphire. High densities of dislocations are formed close to the sapphire interface. From Ref. 29. 
roughly equal number of edge and mixed dislocations accounting for the rest ${ }^{30}$. While long lifetime devices have been fabricated on such defective material, the threading dislocations are still limiting nitride based device development. Dislocations act as non-radiative recombination centers for electron-hole pairs ${ }^{1}$ and reduce the efficiency of such devices. Furthermore, dislocations act as Columbic scattering centers limiting the mobility of carriers in the material and as current leakage paths in device ${ }^{1}$. As a result several techniques have been developed for decreasing the number of dislocation densities in these films such as Epitaxial Lateral Overgrowth (ELOG), Pendo-Epitaxy, and Cantilever Epitaxy ${ }^{1}$.

\subsubsection{Epitaxial Lateral Overgrowth}

Both One Step ELOG and Two Step ELOG methods used in commonly used in MOVPE growth $^{1,30}$. In both techniques, the process begins with a 1-2 $\mu \mathrm{m}$ GaN epilayer grown on a foreign substrate such as sapphire. Next a thin, approximately $100-200 \mathrm{~nm}$, silicon dioxide or silicon nitride layer is deposited. Standard UV photolithography and wet etching techniques are used to create a striped pattern in the oxide along the $<1 \overline{1} 00>\mathrm{GaN}$ direction as shown in Figure 2-11a. This direction is used due to experimental results indicating this direction facilitates faster lateral overgrowth compared stripes oriented along the $<11 \overline{2} 0>\mathrm{GaN}$ direction $^{34}$. Various dimensions of the striped pattern are used, but examples include a $3 \mu \mathrm{m}$ window width and $7 \mu \mathrm{m}$ mask width ${ }^{30}$. In One Step ELOG, the sample is returned to the MOVPE chamber after patterning for re-growth under conditions promoting fast lateral growth. Such growth conditions include high temperature, low pressure, or high V/III ratio ${ }^{1}$. Growth begins in the window region on the exposed $\mathrm{GaN}$ and then extends laterally over the mask region as shown in Figure 2-11b. Eventually the two wing regions coalesce over the mask and result in a smooth film. The oxide mask blocks any dislocations propagating towards the surface resulting in virtually dislocation free material above the mask. However, at the coalescence boundary defects are introduced. The material grown above the window region reflects the quality of the epilayer below and the dislocations continue to propagate to the surface. Before coalescence the growth exhibits a rectangular cross section bounded by the (0001) plane and vertical $\{11 \overline{2} 0\}$ sidewalls as illustrated in Figure 2-11b. The reduction in dislocation density averaged over the entire surface is highly dependent on the ratio of mask width to window width. The larger this ratio, the wider the area of low defect material and thus a lower overall 


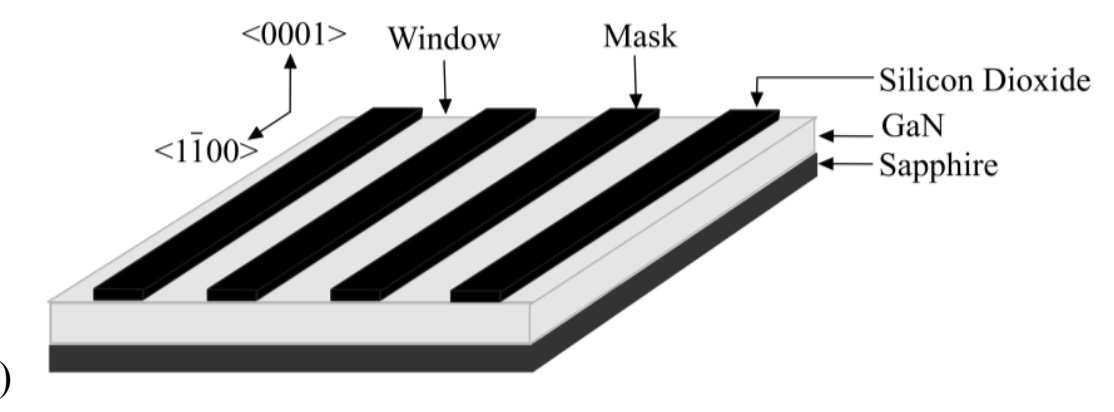

a)
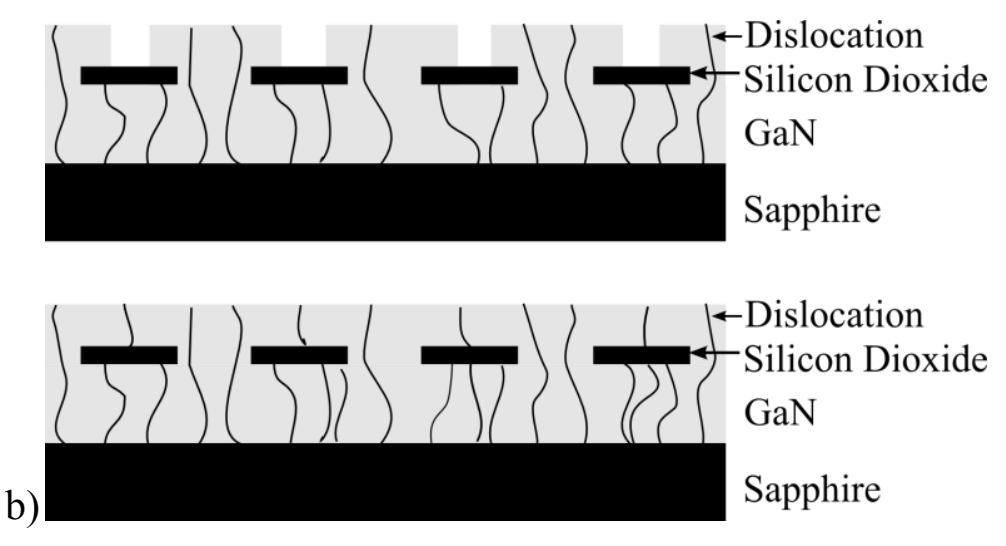

Figure 2-11 - (a) ELOG mask pattern and (b) schematic of One Step Elog Growth.

dislocation density is achieved ${ }^{30}$. With a reported GaN defect density of mid $10^{8} \mathrm{~cm}^{-2}$, it has been observed that using one step ELOG reduces the dislocation density to approximately mid $10^{6}-10^{7} \mathrm{~cm}^{-2}$ on average ${ }^{1}$ as determined by TEM and Atomic Force Microscopy (AFM). Often times, upon coalescence a void is formed along $<1 \overline{1} 00>$ direction but does not impact the quality of subsequently grown material ${ }^{1}$.

In Two Step ELOG ${ }^{1,30,33}$, often called FACELO (Facet Controlled Epitaixal Lateral Overgrowth), the process starts with the same silicon dioxide striped pattern on a GaN epilayer as shown in Figure 2-11a. In the first step, growth conditions are identified to promote vertical growth instead of lateral growth. This is commonly accomplished in MOVPE growth by lowering the growth temperature. The objective is to form well defined structures with triangular cross section bounded by the $\{11 \overline{2} 2\}$ planes as shown in Figure 2-12a. In the second step, the growth conditions are adjusted to promote lateral overgrowth as illustrated in Figure 2-12b. This can include increasing the growth temperature and/or V/III ratio, or by the 

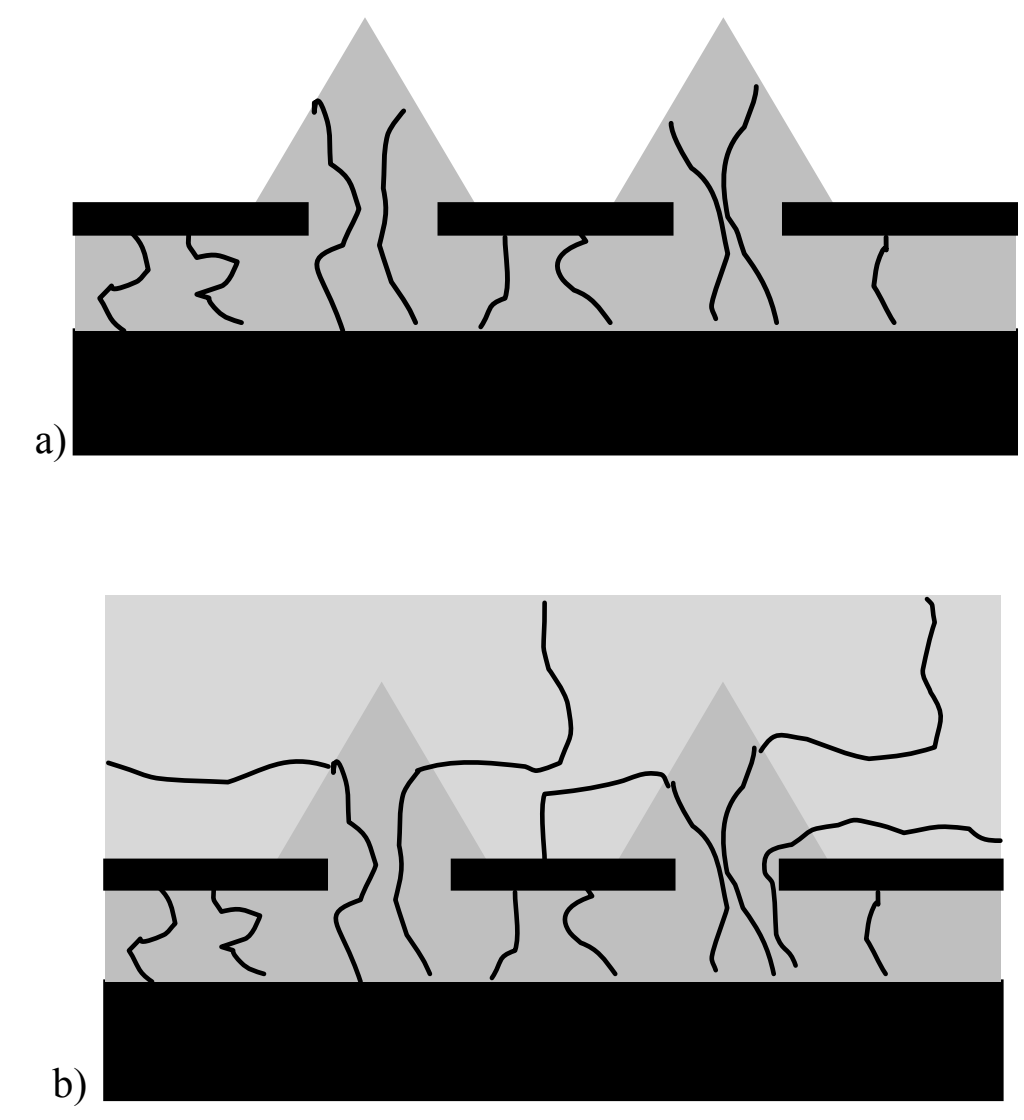

Figure 2-12 - Illustration of Two Step ELOG. a) Step 1 involves the formation of structures with triangular cross section. b) In Step 2, lateral growth is induced to achieve coalescence.

introduction magnesium in the vapor phase ${ }^{30}$. It has been shown that magnesium facilitates the fast lateral growth by suppressing growth along (0001) plane $^{32}$. When the dislocations encounter the $\{11 \overline{2} 2\}$ sidewalls of the structure in the first step, they have been observed to bend $90^{\circ}$ to run parallel with the basal plane and are thus prevented from reaching the surface of the ELOG layer $^{1}$ as shown in Figure 2-12b. All dislocations, edge, screw, or mixed, have been demonstrated to bend ${ }^{33}$. Similarly as described in the One Step process, the dislocations underneath the silicon dioxide mask are also prevented from reaching the surface. At the coalescence boundary, the dislocations that have bent $90^{\circ}$ either are annihilated, bend down toward the substrate or bend up toward the surface ${ }^{33}$. It has been observed that the dislocations mainly appear in the center of the mask area and very few appear in the center of the window ${ }^{33}$. The total dislocation density has been reduced with this technique from the mid $10^{8} \mathrm{~cm}^{-2}$ to $10^{6}$ $\mathrm{cm}^{-2}$. The bending of the dislocations is often attributed to the stress the dislocation encounters 
as it threads toward a surface that is not normal to the direction of propagation. When the dislocation is sufficiently close to the surface, the dislocation bends to adopt a new direction which minimizes the stress on the free surface ${ }^{30}$.

Due to the fact that in the Two Step ELOG process, the formation of the structures with a triangular cross section is critical, the effects of the various growth parameters on the morphology of the Selective Area Growth (SAG) of $\mathrm{GaN}$ on a striped dielectric pattern in rotational environments is well reported in the literature. The majority of these studies investigated the effect of growth conditions for ELOG. Before coalescence, ELOG growths typically result in three dimensional structures bounded by some combination of the (0001), $\{11 \overline{2} 2\}$, and $\{11 \overline{2} 0\}$ facets and exhibit square, triangular, or trapezoidal cross sections. Growth parameters investigated include temperature, pressure, V/III ratio, and the pattern fill factor. Low growth temperatures result in $\{1 \overline{1} 01\}$ sidewalls and poor surface morphology due to the decreased migration of gallium species. Increased growth temperature results in $\{11 \overline{2} 2\}$ sidewalls and improved morphology on the (0001) surface. Even higher growth temperatures lead to the formation of $\{11 \overline{2} 0\}$ sidewalls $^{34}$. Sidewall formation can be attributed to the number of dangling bonds on each surface. The $\{11 \overline{2} 0\}$ plane has fewer dangling bonds when compared to the $\{11 \overline{2} 2\}$ plane and therefore is energetically favored under high temperatures ${ }^{34,35}$. The local V/III ratio increases as the ELOG growth laterally extends over the mask and the concentration of gallium species diffusing from the mask to the growth location decreases which also aids in the transformation from $\{11 \overline{2} 2\}$ facets to $\{11 \overline{2} 0\}$ facets $^{36}$. Furthermore, it has been demonstrated that flow modulation of ammonia $\left(\mathrm{NH}_{3}\right)$ can be used to control the morphology ${ }^{37}$. As the $\mathrm{NH}_{3}$ interruption time increased, the lateral growth rate increased and is credited to the enhanced diffusion of the gallium species during the $\mathrm{NH}_{3}$ interruption.

The stress relaxation in ELOG templates has been previously reported. ELOG techniques have been used in HVPE GaN to eliminate cracking indicating a significant change in the strain profiles of ELOG growth ${ }^{38}$. Furthermore, modeling and experimental analysis has been reported on the evolution of strain in ELOG templates ${ }^{39,40}$ and indicate there is a relaxation of misfit strain which arises in GaN grown on sapphire ${ }^{40}$. 


\subsubsection{Aluminum Nitride and Aluminum Gallium Nitride Growth}

Compared to $\mathrm{GaN}$, the growth of $\mathrm{Al}$ containing III-Nitride alloys via MOVPE is challenging due to the high reactivity of Al containing precursors, low mobility of Al species, and finally the large lattice and thermal mismatch between epilayers ${ }^{16,41,42,43}$. The high reactivity of $\mathrm{Al}$ containing precursors is minimized by separating the precursors in the growth chamber until before the growth site. In the MOVPE system used in this work, this is accomplished by the quartz plating separating the $\mathrm{MO}$ and the ammonia line in the growth chamber as shown in Figure 3-4. Furthermore, $\mathrm{Al}$ containing alloys are grown at a relatively low growth pressures when compared to GaN to minimize parasitic reactions ${ }^{41,42}$. The low mobility of $\mathrm{Al}$ species is generally accommodated by using high growth temperatures.

As shown in Table 2-2, AlN and GaN are characterized by approximately $2.4 \%$ lattice mismatch. AlN grown coherently on $\mathrm{GaN}$ is under tensile strain and often leads to the formation of misfit dislocations and even cracking ${ }^{3}$. A hexagonal crack network is very commonly observed in $\mathrm{Al}_{x} \mathrm{Ga}_{1-x} \mathrm{~N}$ alloys with $\mathrm{Al}$ concentrations greater than $20 \%$ when grown via MOVPE or MBE on GaN layers ${ }^{16}$. When grown on silicon substrates, the thermal mismatch becomes the predominant problem leading to cracking of the material during cool down ${ }^{44}$. As material cracking is detrimental to device fabrication, several techniques have been reported to

Table 2-2 - Lattice parameters and thermal expansion coefficients of III-Nitride Alloys ${ }^{2,43,45}$

\begin{tabular}{|c|c|c|c|c|c|c|}
\hline & \multicolumn{2}{|c|}{$\begin{array}{c}\text { Lattice } \\
\text { Parameters }\end{array}$} & \multicolumn{2}{|c|}{$\begin{array}{c}\text { Thermal Expansion } \\
\text { Coefficient }\end{array}$} & $\begin{array}{c}\text { Lattice Parameter at } \\
\text { Typical Growth } \\
\text { Temperatures }\left(\mathbf{1 0 0 0}{ }^{\circ} \mathbf{C}\right)\end{array}$ \\
\hline Material & $\mathbf{a}(\mathbf{A})$ & $\mathbf{c}(\mathbf{A})$ & $\mathbf{a}\left(\mathbf{K}^{-1}\right)$ & $\mathbf{c}\left(\mathbf{K}^{-1}\right)$ & $\mathbf{a}(\AA)$ & $\mathbf{c}(\mathbf{A})$ \\
\hline AlN & 3.11 & 4.98 & $4.2 \times 10^{-6}$ & $5.3 \times 10^{-6}$ & 3.123 & 5.006 \\
\hline GaN & 3.189 & 5.185 & $5.59 \times 10^{-6}$ & $3.17 \times 10^{-6}$ & 3.206 & 5.201 \\
\hline InN & 3.54 & 5.76 & $5.6 \times 10^{-6}$ & $3.8 \times 10^{-6}$ & N/A & N/A \\
\hline Sapphire & 4.758 & 13.0003 & $7.5 \times 10^{-6}$ & $8.5 \times 10^{-6}$ & 4.793 & 13.108 \\
\hline Rotated Sapphire & 2.379 & 13.0003 & $7.5 \times 10^{-6}$ & $8.5 \times 10^{-6}$ & 2.396 & 13.108 \\
\hline
\end{tabular}


accommodate the strain and minimize or eliminate the cracking. Two of the most commonly reported techniques include the use of AIN interlayers ${ }^{46,47,48,49,50}$ and $\mathrm{Al}_{x} \mathrm{Ga}_{1-x} \mathrm{~N}$ graded buffers ${ }^{44}$. The use of surfactants and digital structures as pursued in this work, are less widely studied in III-Nitride alloys grown via MOVPE.

\subsubsection{Aluminum Nitride Interlayers}

AlN interlayers refer to the use of thin AlN layers, typically grown at low temperatures, inserted before the strained structure as illustrated in Figure 2-13. Typical interlayer thicknesses range from approximately10 $\mathrm{nm}$ to $30 \mathrm{~nm}^{50}$. For thick $\mathrm{Al}_{x} \mathrm{Ga}_{1-x} \mathrm{~N}$ alloys, the AlN interlayer has been proven an effective means of eliminating the crack formation. The commonly accepted explanation is that the low growth temperature results in incoherent AlN growth ${ }^{46}$. This effectively de-couples all subsequent layers from that underneath by creating a new seed, or template, for growth ${ }^{46,48,50}$. On the contrary, other observations have indicated that micro-cracks are formed in the AlN interlayer during growth and cause compression in subsequent $\mathrm{Al}_{x} \mathrm{Ga}_{1-x} \mathrm{~N}$ layers to prevent cracking ${ }^{49}$. The $\mathrm{Al}_{x} \mathrm{Ga}_{1-x} \mathrm{~N}$ overgrows the cracks which are buried

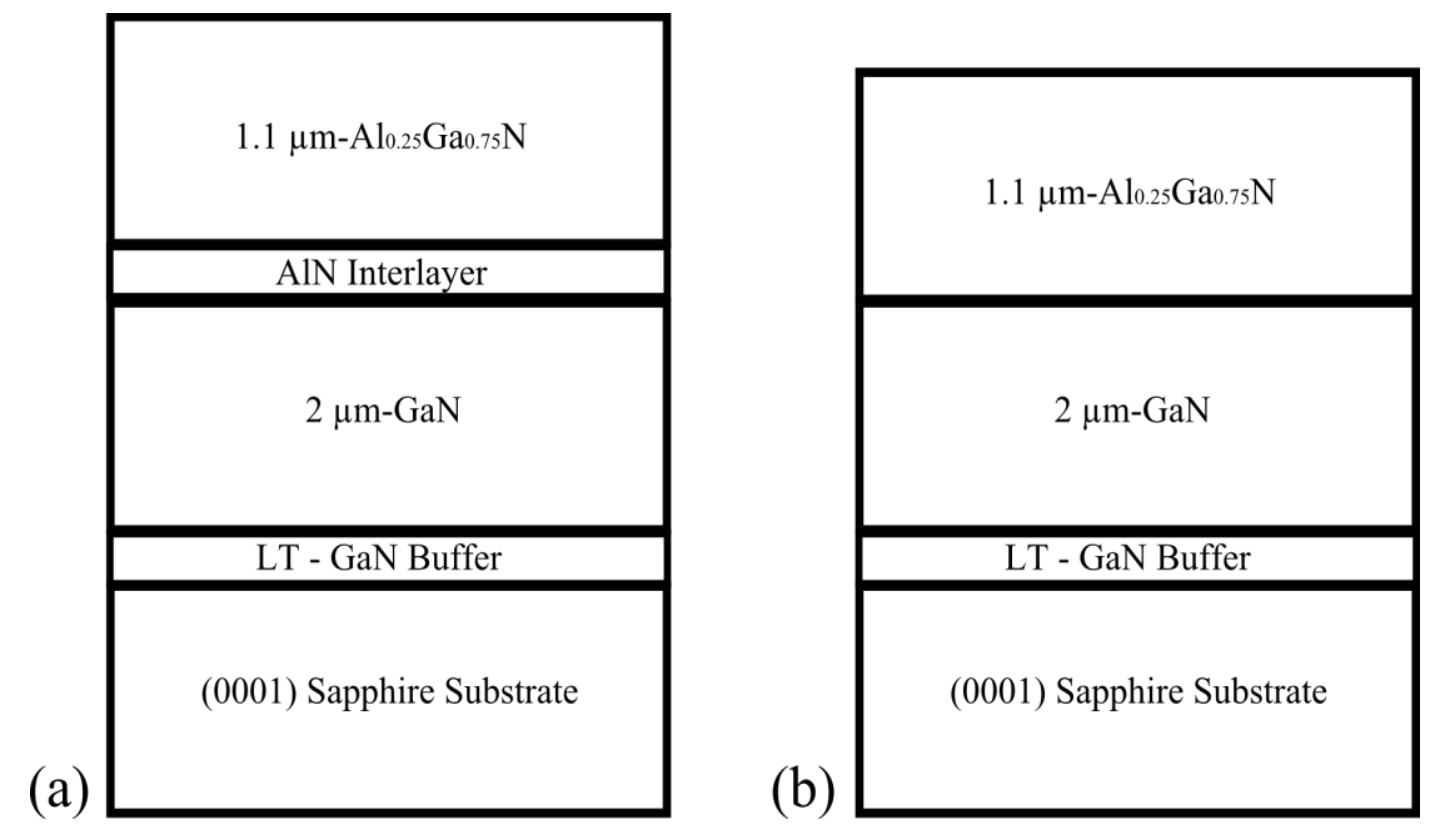

Figure 2-13 - Schematic of $A l_{x} G a_{1-x} N$ layers grown on sapphire (a) with and (b) without AlN interlayers. Modified from Ref. 48. 
in the interlayer ${ }^{49}$. As the growth conditions, such as temperature and V/III ratio, greatly influence the cracking in the interlayer, it can be used to adjust the strain in the subsequent $\mathrm{Al}_{x} \mathrm{Ga}_{1-x} \mathrm{~N}$ layer ${ }^{49}$.

\subsubsection{Aluminum Gallium Nitride Graded Buffer Layers}

$\mathrm{Al}_{x} \mathrm{Ga}_{1-\mathrm{x}} \mathrm{N}$ graded buffer layers are commonly used to accommodate the thermal mismatch between III-Nitride layers and silicon substrates ${ }^{44}$. In graded buffer layers, the $\mathrm{Al}$ concentration in a single $\mathrm{Al}_{x} \mathrm{Ga}_{1-x} \mathrm{~N}$ layer is gradually increased during the growth of the layer. This results in the gradual change in the lattice parameter. The elimination in cracking upon cool down is attributed to the introduction of compressive strain through the graded buffer layer ${ }^{44}$.

\subsubsection{Surfactants}

Surfactants are impurities that collect and "float" on the surface during growth. They typically are not incorporated in the growth due to low solubility ${ }^{26}$. By saturating the surface they allow the species of interest to diffuse to a more energetically favorable location before being incorporated into the material ${ }^{26}$ as illustrated in Figure 2-14. Common surfactants used

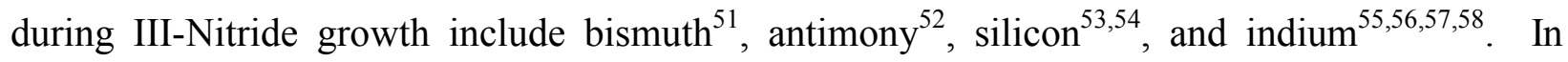
MBE growth of dilute nitrides ( $\mathrm{GaAsN})$, both bismuth and antimony lead to decreased surface roughness. Additionally, bismuth enabled increased nitrogen incorporation. As compared to MBE, surfactant use in MOVPE is somewhat less common due to potential chemical reactions with the precursors ${ }^{26}$. Common surfactants include those which are introduced into the reactor for conventional growth purposes, such as silicon and indium which are used for $n$-type doping and $\operatorname{In}_{x} \mathrm{Ga}_{1-x} \mathrm{~N}$ growth respectively. Silicon is used as a surfactant in both MOVPE ${ }^{54}$ and $\mathrm{MBE}^{53}$ growth but has been linked to a transition from 2D growth to 3D growth. This has potential applications for quantum dot fabrication. Likewise, indium has been employed in both $\mathrm{MOVPE}^{57,58}$ and MBE ${ }^{55,56}$. In contrast to silicon, it has promoted 2D growth in III-Nitrides and resulted in improved surface and crystal morphology. In MBE growth, improved photoluminescence and surface morphology was observed by using a small indium flux during growth of $\mathrm{GaN}^{56}$. More recently, indium has been used to promote the vertical growth of nano

wires via $\mathrm{MBE}^{59}$. The indium promotes the diffusion of the gallium species but has negligible incorporation into the lattice. In GaN growth via MOVPE, indium has decreased surface roughness and led to a relaxation in the film ${ }^{57,59}$ as shown in Figure 2-15. In this figure, a 

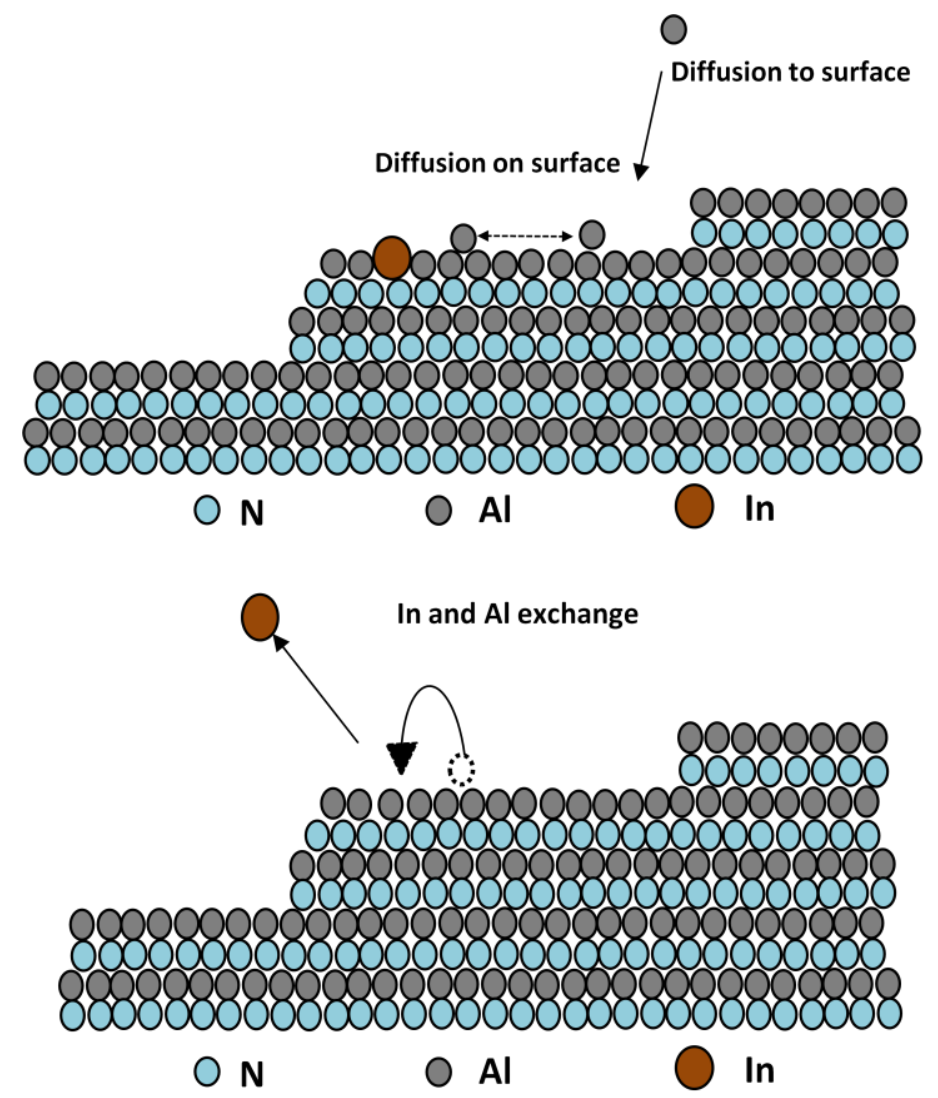

Figure 2-14 - Illustration of surfactant effects of indium during AlN growth. Indium floats on the surface to allow the Al species to diffuse to the energetically favorable location where it is incorporated into the lattice.

dramatic decrease in surface roughness can be observed after the initial introduction of indium into the growth. In Figure 2-15b, the arrow indicates the direction of increasing indium flow. $\mathrm{Up}$ to certain point, the $\mathrm{GaN} a$ - and $c$-lattice parameter approach that of bulk material indicating relaxation in the film. For large indium flows, the deviation from the line is explained by the formation of $\operatorname{In}_{x} \mathrm{Ga}_{1-x} \mathrm{~N}$ alloys. Up to that point, it is assumed that the indium is not incorporated into the lattice positions, but instead incorporated at defect sites. This serves to reduce the energy of the system, relieve strain, and also to pin dislocations to improve the crystal quality $^{57,59}$. In AlN growth, indium has also been used a surfactant to improve surface morphology ${ }^{60}$ as shown in Figure 2-16a. In thin AlN layers for use in multi-quantum wells, the use of indium changed the surface growth kinetics such that a wavy surface resulted. This wavy surface allows for increased storage of excess energy due to the lattice mismatch and prevented crack formation. Furthermore, the decreased FWHM of the rocking curve shown in Figure 2-16b implies improved crystal quality. 

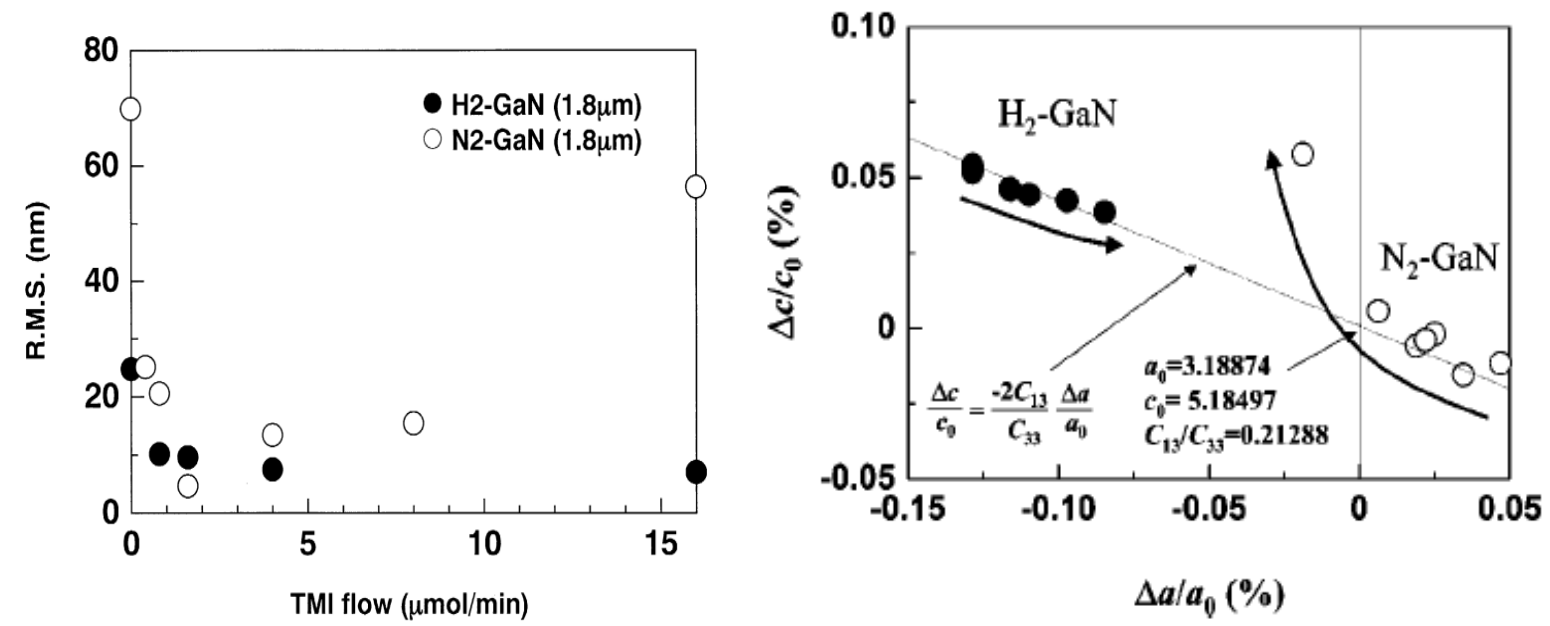

Figure 2-15 - (a) Surface roughness of GaN grown with varying amounts of indium. (b) $\Delta c / c_{o}$ as a function of $\Delta a / a_{0}$. The dashed line denotes the relationship between $\Delta c / c 0$ and $\Delta a / a 0$ estimated by calculation. The direction of the arrows indicates increasing indium flow rate. From Ref 57,58.

Other studies have simply reported a decrease in the surface roughness ${ }^{61}$, but not necessarily a change in surface kinetics. Although the reasons for the strain relaxing effects of indium are not well agreed upon in the literature, the effects have been reported by several research groups.

\subsubsection{Digital Alloys}

Digital alloy growth, often called short period superlattices (SPSL), consists of layers of binary or ternary alloys with layer thicknesses of a few monolayers ${ }^{62}$ as illustrated in Figure 2-17. It has been a very effective means of growing ternary and quaternary alloys in a number of material systems including the arsenides ${ }^{63}$, phosphides $^{64}$, antimonides ${ }^{65}$, and nitrides ${ }^{41,42,66,67,68}$. For example, within the III-Nitride system, the digital growth Aluminum Gallium Nitride $\left(\mathrm{Al}_{\mathrm{x}} \mathrm{Ga}_{1-\mathrm{x}} \mathrm{N}\right)$ consists of the repetition of $\mathrm{m}$ monolayers of AlN followed by $\mathrm{n}$ monolayers of GaN (represented as $\left.(\mathrm{AlN})_{\mathrm{m}}(\mathrm{GaN})_{\mathrm{n}}\right)$. From the growth perspective, digital techniques have presented many advantages over conventional random alloy growth. Digital growth provides an effective means of accurately controlling alloy composition with the number of monolayers in each layer ${ }^{63}$ and allowing for very abrupt changes in the concentration profile. Furthermore, it is also often employed to alleviate material insolubility challenges. Effective digital 

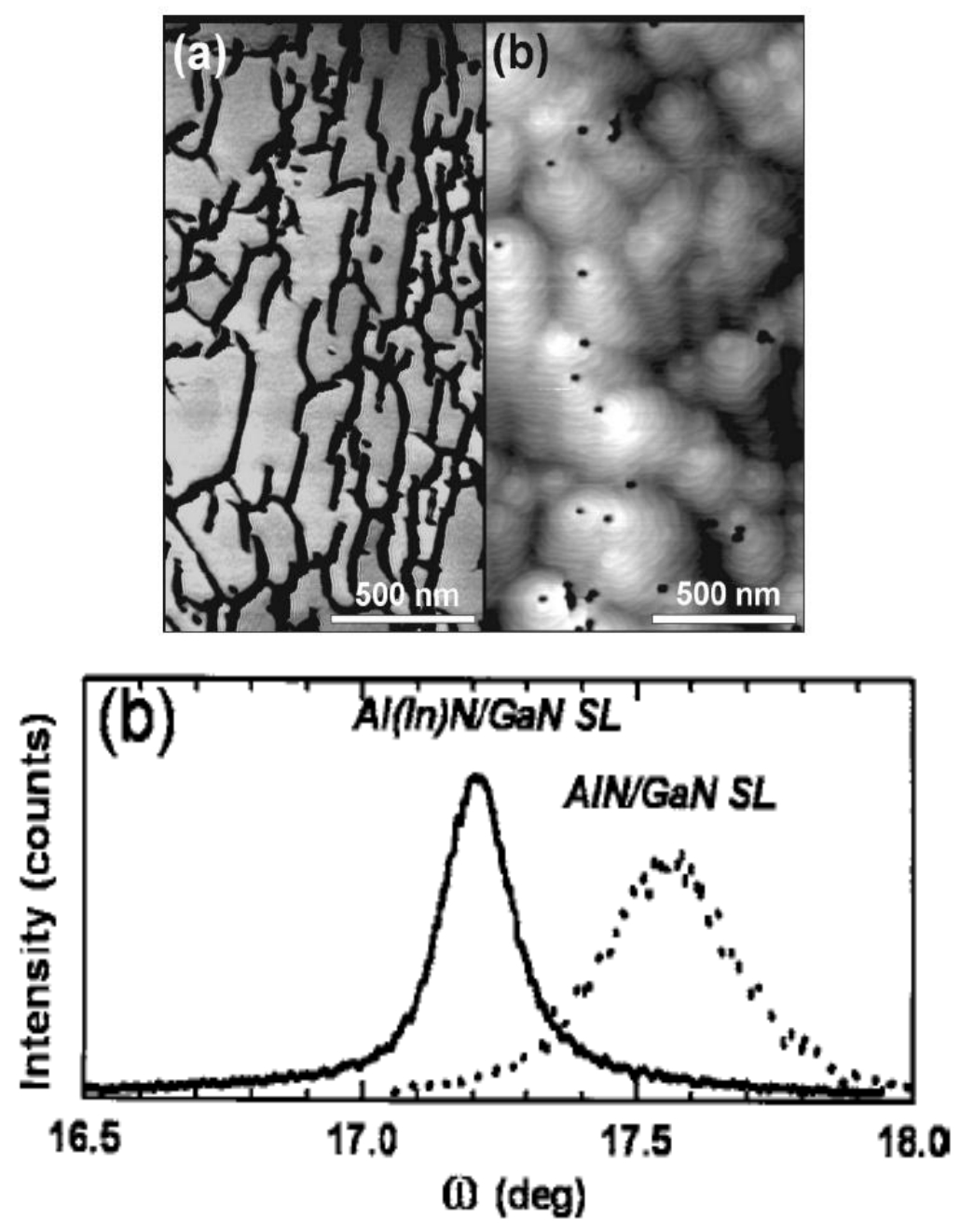

Figure 2-16 - (a) AFM Image of AlN/GaN MQW(right) and Al(In)N/GaN MQW(left). (b) $X R D$ rocking curve around $O^{\text {th }}$ order satellite peak for AlN/GaN MQW structureswith and without indium. From Ref. 60.

$(\mathrm{GaInAs})_{\mathrm{m}}(\mathrm{GaNAs})_{\mathrm{n}}$ alloys have been used to overcome the nitrogen incorporation challenge in the growth of dilute nitrides ${ }^{69}$ while digital techniques have also been used in AlInGaN alloys to increase indium incorporation ${ }^{68}$. Short period superlattices have also been used for strain engineering in the III-Nitride system to reduce tensile $\operatorname{strain}^{70,71}$. As expected, along with the composition, the electronic properties of the material can also be finely tuned using this technique $^{64}$. For the most part, digital techniques are more commonly studied in MBE than MOVPE. In MBE, the technique is very attractive due to the fact that the composition is dependent on shutter timing not incident flux. However, it has been a proven to be a viable 


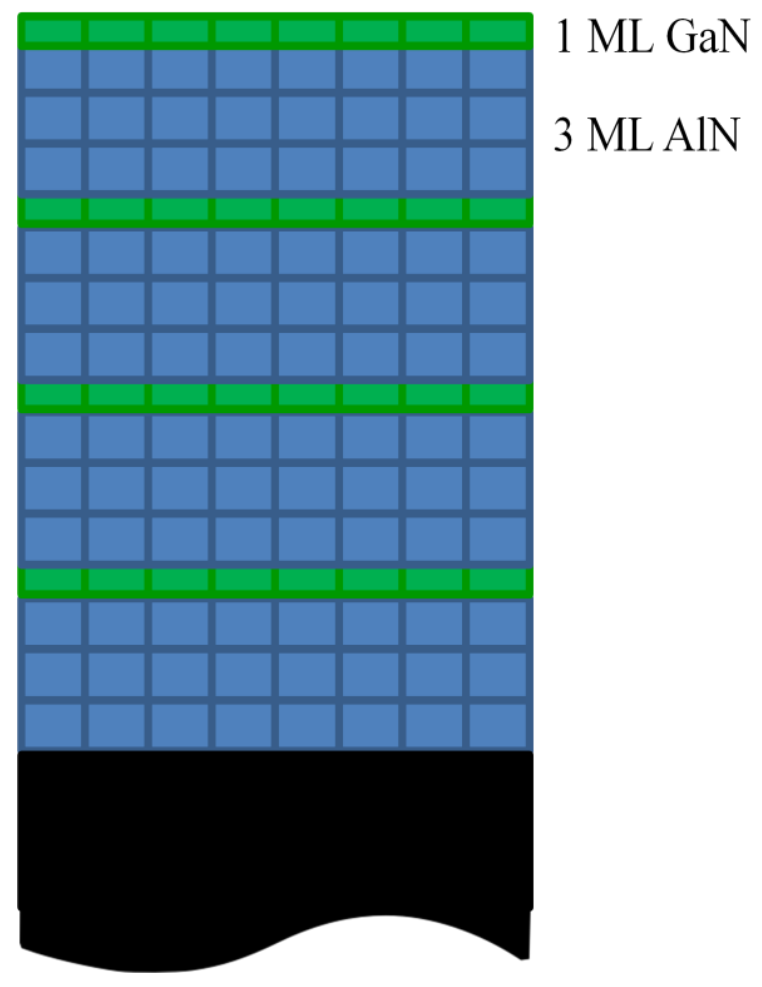

Figure 2-17 - Illustration of $A l_{0.75} G a_{0.25} \mathrm{~N}$ digital alloy comprised of $1 \mathrm{ML}$ of GaN and $3 \mathrm{ML}$ of AlN.

means of alloy growth via MOVPE ${ }^{41,42,66}$, but still requires significant exploration.

Initial reports of $(\mathrm{AlN})_{\mathrm{m}}(\mathrm{GaN})_{\mathrm{n}}$ digital alloys via MOVPE proved it was an effective means of engineering the $\mathrm{Al}$ composition and electronic properties of the films ${ }^{66}$. The most recent work using MOVPE growth is employing a Modulated Precursor Epitaxy Growth $(\mathrm{MPEG})^{41,42}$. In this technique the group III and $\mathrm{V}$ precursors are supplied to the growth chamber in separate pulses. High quality films have been achieved, however, for alloys with large Ga content, surface roughening and inhomogeneous composition were observed. This inhomogeneity was eliminated by growing an AIN MPEG monolayer followed by a conventional $\mathrm{Al}_{\mathrm{x}} \mathrm{Ga}_{1-\mathrm{x}} \mathrm{N}$ random alloy monolayer ${ }^{41,42}$.

It should be recognized that the strain formation and distribution in digital alloys is anticipated to be considerably different than in its random $\mathrm{Al}_{x} \mathrm{Ga}_{1-x} \mathrm{~N}$ counterpart. The biaxial strain in random alloys often leads to a composition pulling effect and thereby a non-uniform composition distribution within a single layer ${ }^{72}$. Sufficient in-plane strain, as observed in high Al content random alloys on GaN epilayers, results in cracking $^{3}$. However, in short period 
superlattices, the strain is distributed between the AlN and GaN layers yielding a different strain profile ${ }^{73}$. As strain management is critical in device design and operation, it is important to understand the strain formation in these digital structures over the entire compositional range in order to fully utilize them in device fabrication.

\subsection{Distributed Bragg Reflectors}

As previously mentioned, this work targets nitride based DBR fabrication. Quarter wavelength DBRs are long period superlattice structures comprised of alternating layers of materials with differing refractive indices ${ }^{6}$ as shown in Figure 2-18. DBRs are designed to be wavelength specific mirrors by making each layer in the superlattice a quarter wavelength thick. In other words, the thickness of each layer, $s$, is given by

$$
S=\frac{\lambda_{o}}{4 n}
$$

In which $\lambda_{\mathrm{o}}$ is the target wavelength for reflection and $n$ is the refractive index of the material at the target wavelength. At each interface light is reflected according to Fresnel's equation. The equations for light perpendicular to the plane of incidence and parallel to the plane of incidence are shown in the following equations ${ }^{6}$ :

$$
\begin{aligned}
& r_{\perp}=\frac{n_{1} \cos (\theta)-n_{2} \cos (\theta)}{n_{1} \cos (\theta)+n_{2} \cos (\theta)} \\
& r_{\|}=\frac{n_{2} \cos (\theta)-n_{1} \cos (\theta)}{n_{2} \cos (\theta)+n_{1} \cos (\theta)}
\end{aligned}
$$

For light normal to the interfaces in the DBR, these equations can be simplified to the following:

$$
r=r_{\perp}=r_{\|}=\frac{n_{1}-n_{2}}{n_{1}+n_{2}}
$$

Light reflecting from a material of lower refractive index $\left(n_{2}>n_{1}\right)$ will undergo a $180^{\circ}$ phase change as indicated by the negative value of the equation. The quarter wavelength thickness of each layer allows the reflections of that wavelength to constructively interfere resulting in high reflectivity. 
In the III-Nitride system, the DBRs are critical to the fabrication of LEDs. In particular, DBRs can be used to enhance light extraction by reflecting the light that is transmitted from the backside of the device or in Resonant Cavity LEDs (RCLEDs). RCLEDs have enhanced efficiency, spectral purity, directionality and bandwidth ${ }^{74}$ compared to regular planar LEDs. In the III-Nitride system, $\mathrm{Al}_{x} \mathrm{Ga}_{1-x} \mathrm{~N}$ and $\mathrm{GaN}$ are pursued as materials due to the large difference in refractive index ${ }^{75}$. Compared with dielectric based DBRs, III-Nitride structures will often consist of 20 to more periods ${ }^{75}$ in order to obtain high reflectivity as shown in Figure 2-19.

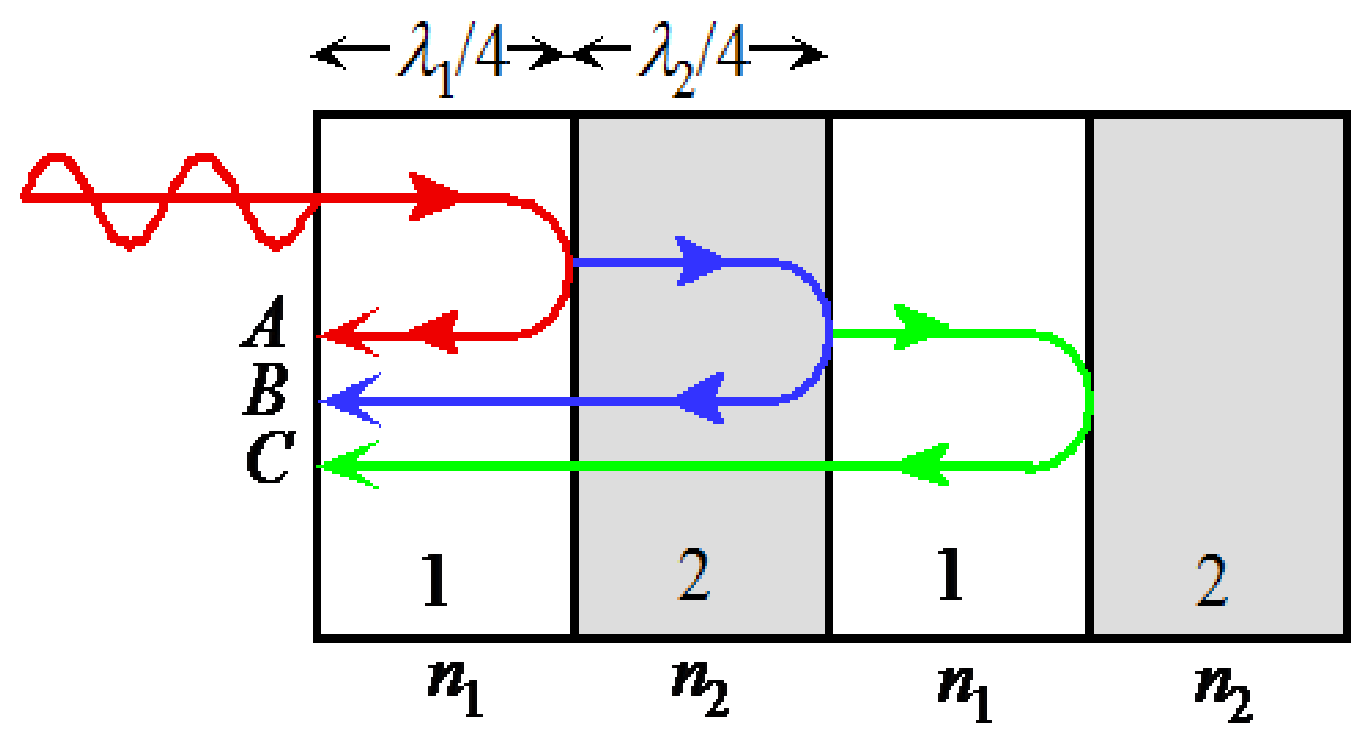

(C) 1999 S.O. Kasap, Optoelectronics(Prentice Hall)

Figure 2-18 - Illustration of DBR. Layer 1 has refractive index $n_{1}$ and layer 2 has refractive index $n_{2}$. Image from Ref. 6. 


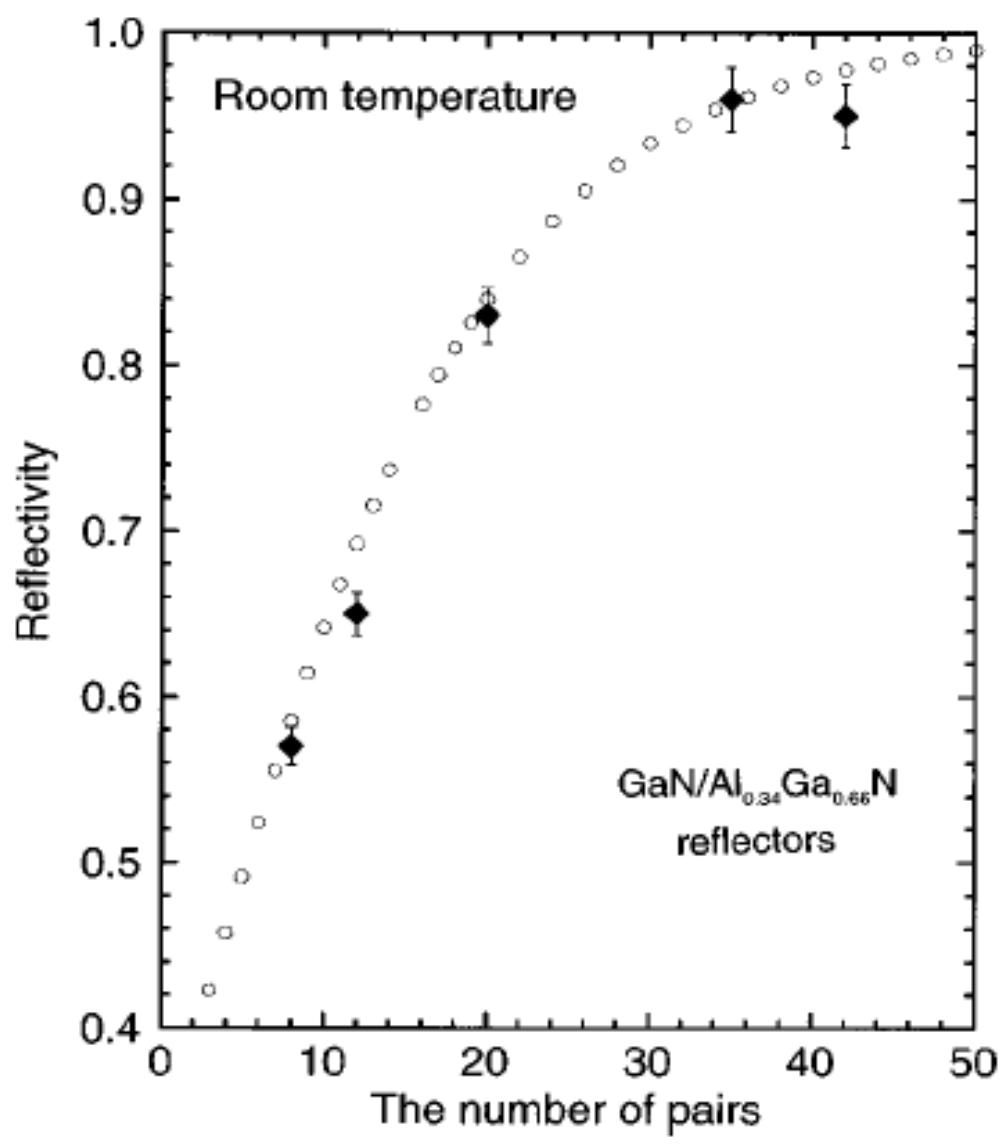

Figure 2-19 - Reflectivity of $A l_{x} G a_{1-x} N / G a N D B R$ with increasing number of periods. From Ref. 75.

Due to the large lattice and thermal mismatch between AlN and GaN, a well defined network of cracks has been reported in both AIN/GaN DBRs grown via Metal Organic Vapor Phase Epitaxy (MOVPE) ${ }^{76}$ and Molecular Beam Epitaxy (MBE) ${ }^{77}$ on sapphire substrates. Several different techniques have been investigated in order to reduce or eliminate cracking, such as using $\mathrm{Al}_{\mathrm{x}} \mathrm{Ga}_{1-\mathrm{x}} \mathrm{N}^{75}$ or $\mathrm{Al}_{\mathrm{x}} \mathrm{In}_{1-\mathrm{x}} \mathrm{N}^{80,81,82}$ in place of AlN to reduce lattice mismatch, inserting superlattice layers either before ${ }^{78}$ or within the DBR structure ${ }^{76}$, and also the use of asymmetric layers ${ }^{77}$. These techniques have been successful in eliminating or reducing the cracking, but not without impacting the fabrication and reflectivity of the DBR. The use of $\mathrm{Al}_{x} \mathrm{Ga}_{1-x} \mathrm{~N}$ or $\mathrm{Al}_{x} \mathrm{In}_{1-x} \mathrm{~N}$ instead of AlN decreases the contrast in refractive index which then requires a larger number of periods to achieve equal reflectivity and also reduces the bandwidth. Figure 2-20 shows the change in band width and also increased required number of periods required when using $\mathrm{Al}_{0.35} \mathrm{Ga}_{0.65} \mathrm{~N}$ instead of AlN. 35 pairs of $\mathrm{Al}_{0.35} \mathrm{Ga}_{0.65} \mathrm{~N} / \mathrm{GaN}$ layers ${ }^{75}$ are required in order to 

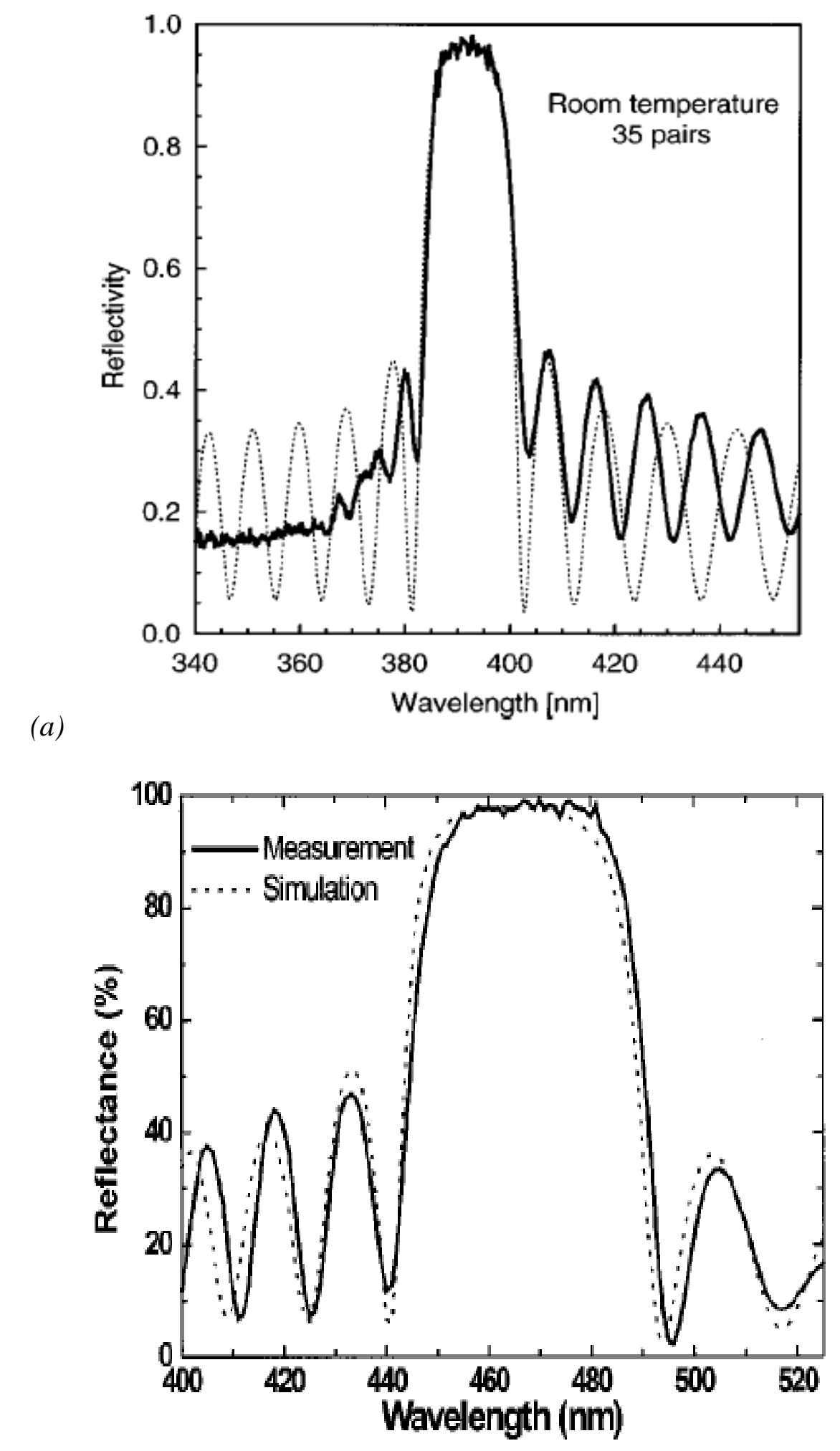

(b)

Figure 2-20 - (a) Reflectivity of 35 pair $A l_{0.34} G a_{0.66} N / G a N$ DBR (From Ref. 75) and (b) reflectivity of 20.5 Period AlN/GaN DBR (From Ref. 77). 
achieved similar reflectivity as that of a 20.5 pair AlN/GaN DBR ${ }^{77}$. Furthermore, as shown in Figure 2-20, the bandwidth decreased from approximately $50 \mathrm{~nm}$ for the AIN/GaN DBR to approximately $20 \mathrm{~nm}$ for the $\mathrm{Al}_{x} \mathrm{Ga}_{1-x} \mathrm{~N} / \mathrm{GaN} \mathrm{DBR}$. $\mathrm{Al}_{x} \mathrm{In}_{1-x} \mathrm{~N} / \mathrm{GaN}$ structures have also been investigated as a viable alternative to $\mathrm{AlN} / \mathrm{GaN}$ structures for DBR fabrication. The most widely noted characteristic of $\mathrm{Al}_{x} \operatorname{In}_{1-x} \mathrm{~N}$ is the very small lattice mismatch to $\mathrm{GaN}$ at $17-18 \%$ In concentrations $^{79}$ as shown in Figure 2-21. The actual lattice matching concentration remains under investigation as there have been reports of deviations from Vegard's law ${ }^{80}$, but the mismatch is approximately $+/-0.5 \%$ for In concentrations ranging from 14 to $22 \%{ }^{81}$. These conditions have been used to alleviate strain issues, and when combined with the $\sim 7 \%$ refractive index contrast to $\mathrm{GaN}$ as shown in Figure 2-22, $\mathrm{Al}_{x} \mathrm{In}_{1-x} \mathrm{~N}$ is an attractive material for use in Distributed Bragg Reflectors (DBRs). Crack-free, 20 period DBRs with approximately 90\% reflectivity $^{79}$ and 40 period DBRs with greater than $99 \%$ reflectivity ${ }^{82}$ have been fabricated with $\mathrm{Al}_{x} \mathrm{In}_{1-x} \mathrm{~N} / \mathrm{GaN}$ grown via Metal Organic Vapor Phase Epitaxy as shown in Figure 2-23. It can be seen that the bandwidth of the reflectivity is between $30-35 \mathrm{~nm}$ in both structures. This is still considerable smaller than that of $\mathrm{AlN} / \mathrm{GaN}$ due to the decrease in refractive index contrast as shown in Figure 2-22. It is also known that these alloys, in particular $\operatorname{In}_{x} \mathrm{Ga}_{1-x} \mathrm{~N}$ and $\mathrm{Al}_{x} \operatorname{In}_{1-x} \mathrm{~N}$, are characterized by phase separation due to a large immiscibility gap giving rise to growth challenges ${ }^{80,83}$. The large difference in the growth temperature between $\operatorname{InN}\left(\sim 600{ }^{\circ} \mathrm{C}\right)$ and AlN $\left(\sim 1100{ }^{\circ} \mathrm{C}\right)$ makes it difficult to obtain high quality material with large In concentrations. Atomic ordering has been observed in both $\mathrm{Al}_{x} \mathrm{Ga}_{1-x} \mathrm{~N}^{84}$ and $\operatorname{In}_{x} \mathrm{Ga}_{1-x} \mathrm{~N}$ alloys ${ }^{85}$, but has not been widely studied in $\mathrm{Al}_{x} \mathrm{In}_{1-x} \mathrm{~N}$ and $\mathrm{Al}_{x} \mathrm{In}_{y} \mathrm{Ga}_{1-x-y} \mathrm{~N}$ alloys. The presence of these micro-structures significantly affects the macroscopic properties of the material and therefore requires further investigation.

As mentioned previously, short period superlattices modify the strain profile within epi layers. As a result, superlattice insertion layers have also been used as an effective means of the relieving the strain in both $\mathrm{Al}_{0.35} \mathrm{Ga}_{0.65} \mathrm{~N} / \mathrm{GaN}$ and $\mathrm{AlN} / \mathrm{GaN} \mathrm{DBR}$ structures. In both cases, the superlattices have been shown to relieve strain and decrease the cracking. Figure 2-24 contains the SEM images, optical microscope images, and reflectivity measurements for $\mathrm{Al}_{0.35} \mathrm{Ga}_{0.65} \mathrm{~N} / \mathrm{GaN}$ DBRs grown by inserting a superlattice before the DBR and also embedded within the $\mathrm{DBR}^{78}$. In this experiment a 40 period $\mathrm{Al}_{x} \mathrm{Ga}_{1-x} \mathrm{~N} / \mathrm{GaN}$ superlattice was used as the strain reducing layer. The maximum $\mathrm{Al}$ concentration in the $\mathrm{Al}_{x} \mathrm{Ga}_{1-x} \mathrm{~N}$ film used in the 


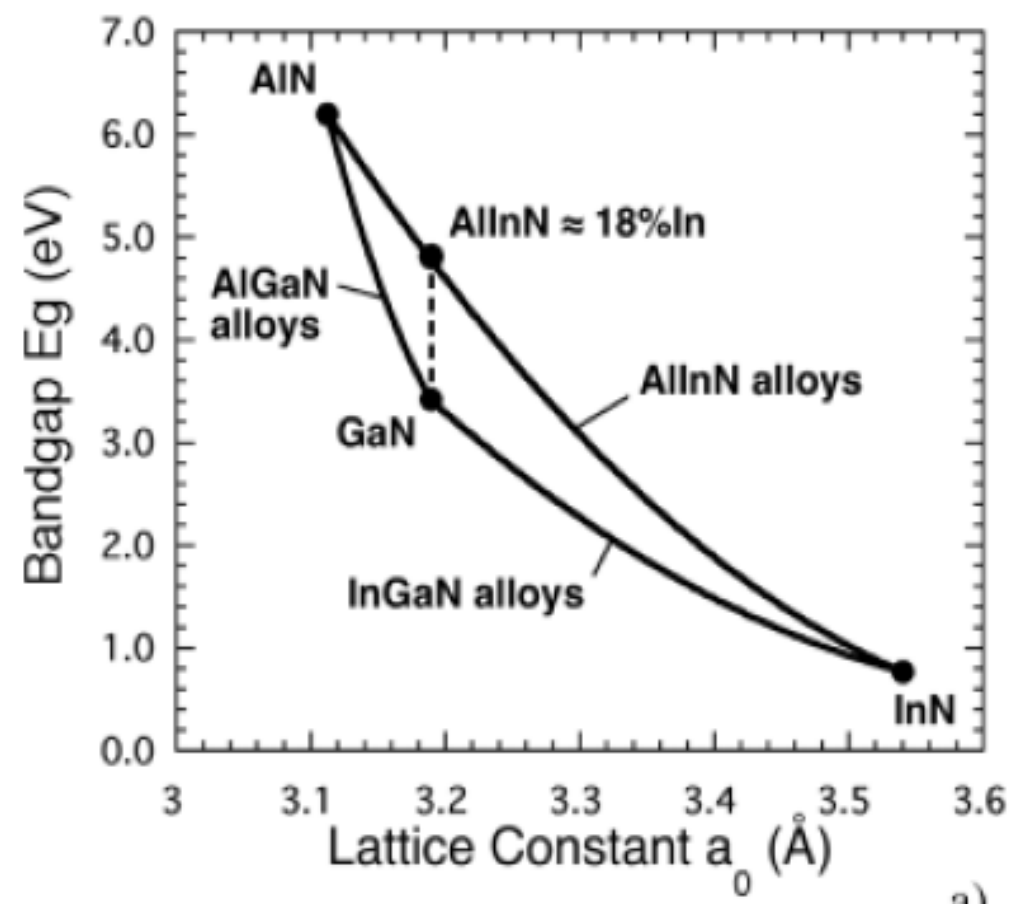

Figure 2-21 - Lattice constant versus bandgap for (Al,Ga,In)N materials. From Ref. 79.

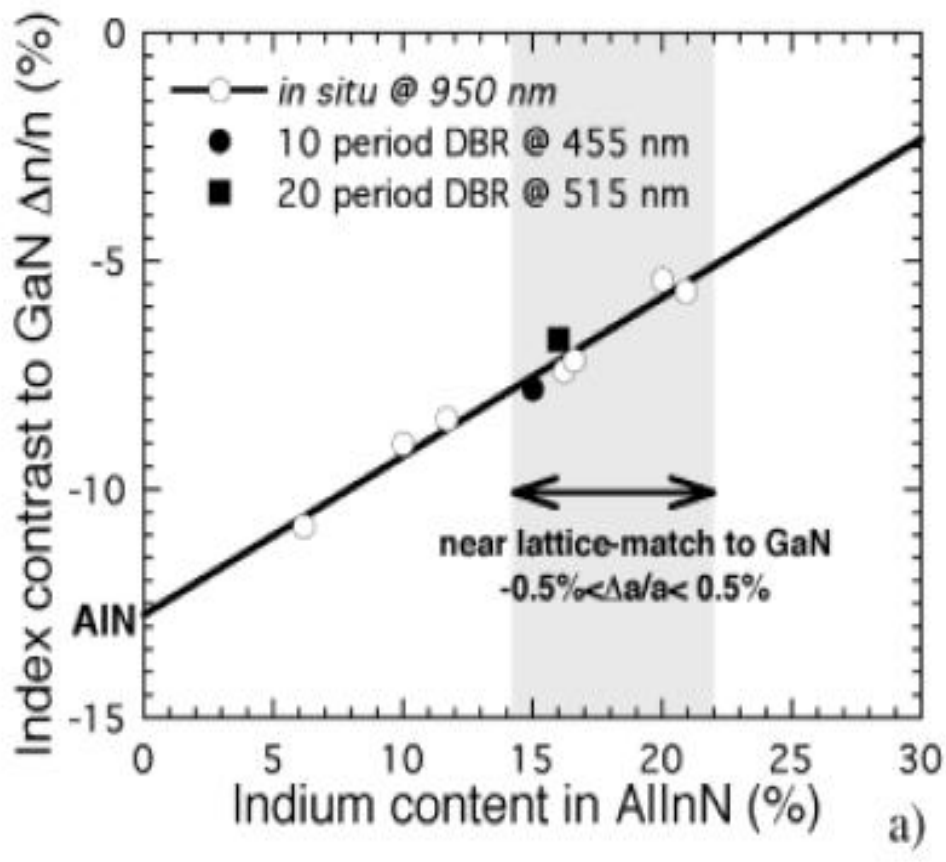

Figure 2-22 - Refractive index contrast of AlInN alloys as a function of the indium concentration. From Ref. 79. 


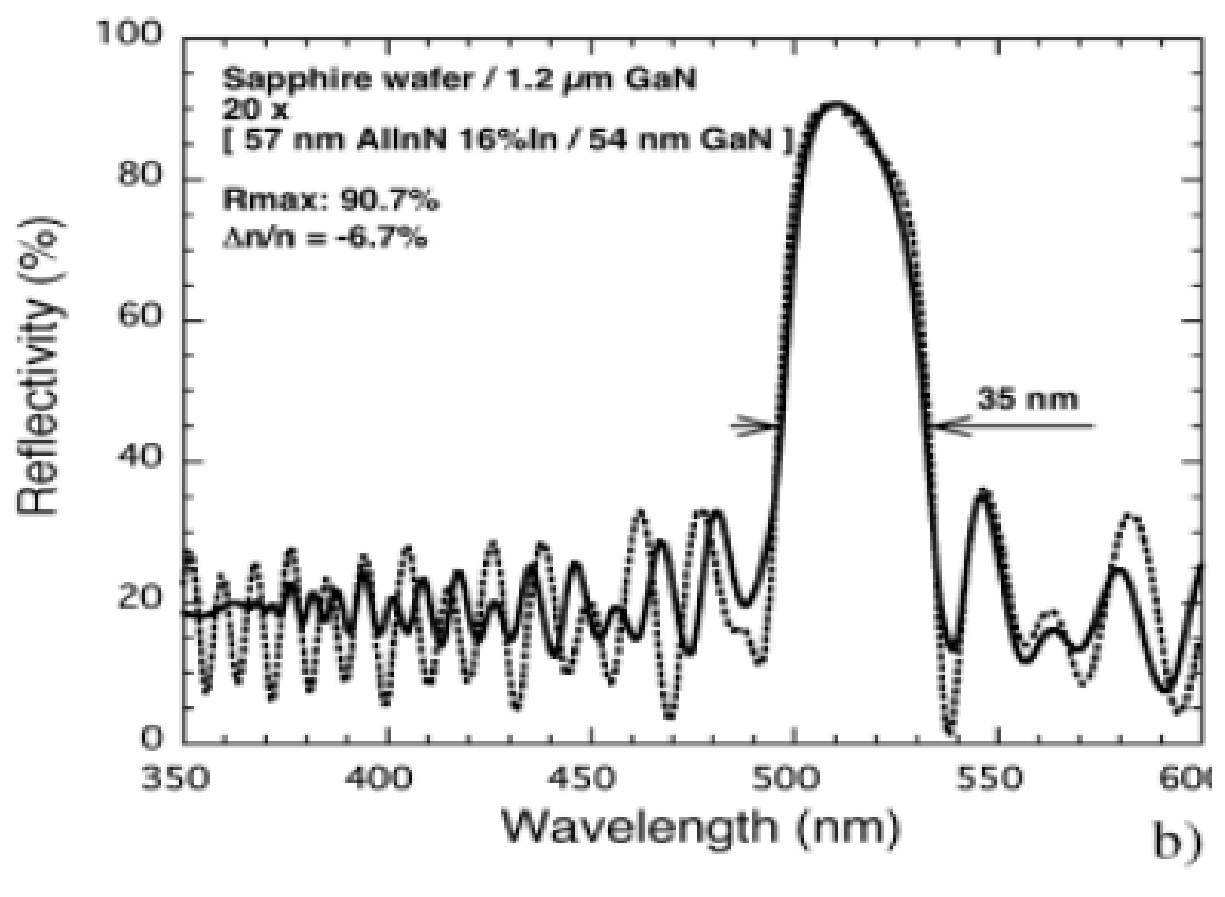

(a)

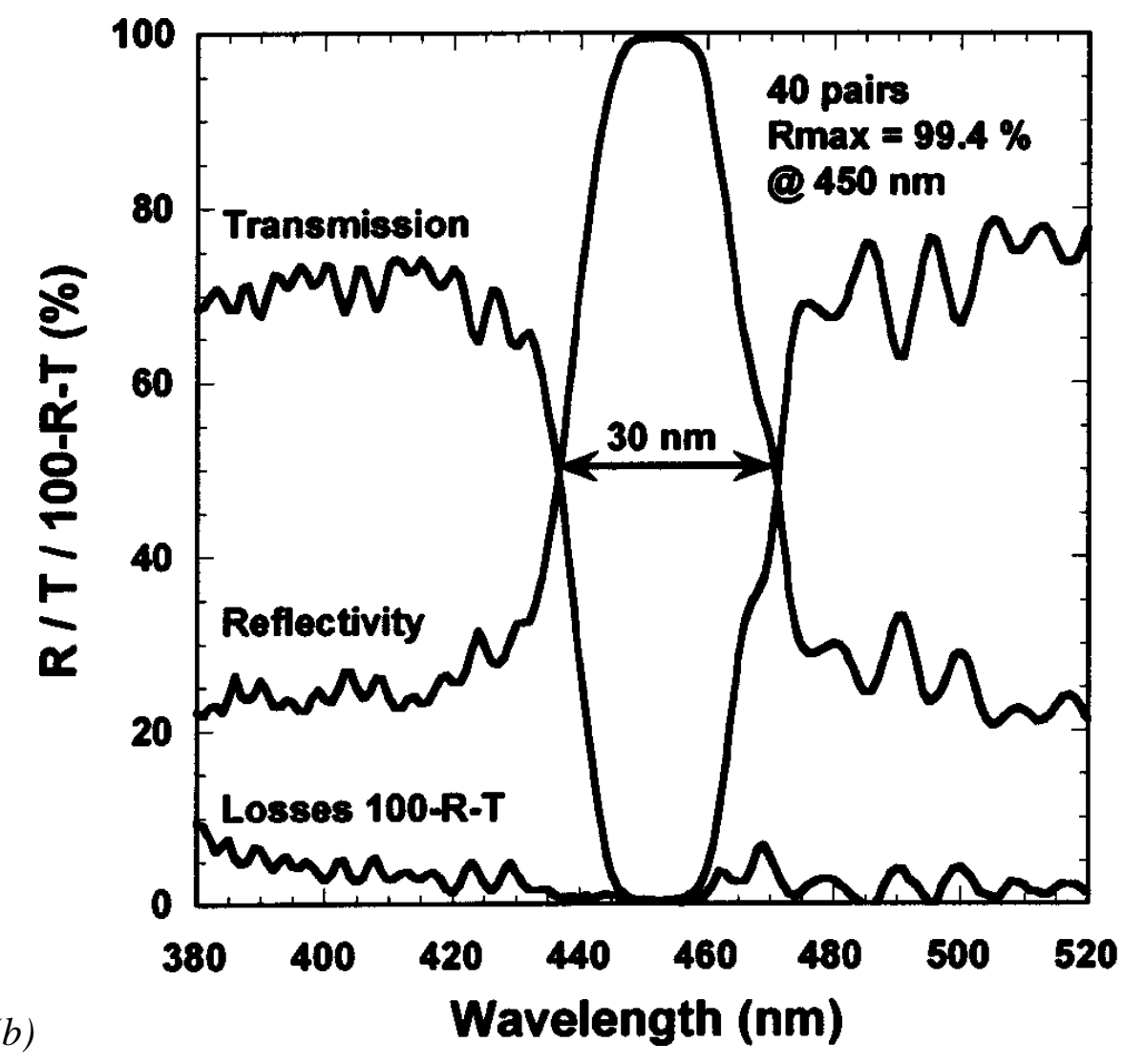

Figure 2-23 - Reflectivity spectra of (a) 20 and (b) 40 period $A l_{x} I_{1-x} N / G a N$ DBR. From Ref. 79,82 . 
superlattice was less than $10 \%$. There was not a complete elimination of the cracking observed, but a considerable reduction. However, this group reported interference in the reflectivity measurements as a result of the embedded superlattice layers. Other reports using superlattice insertion layers have completely suppressed cracking in 20 pair AlN/GaN DBR structures ${ }^{76}$. In particular, 5.5 periods of AlN/GaN superlattices have a total thickness equal to a half wavelength were inserted after every five periods of the DBR as shown in Figure 2-25. This technique was used to obtain DBRs having $>90 \%$ reflectivity centered around $400-440 \mathrm{~nm}$. No interference in the reflectivity spectra was reported in this work.
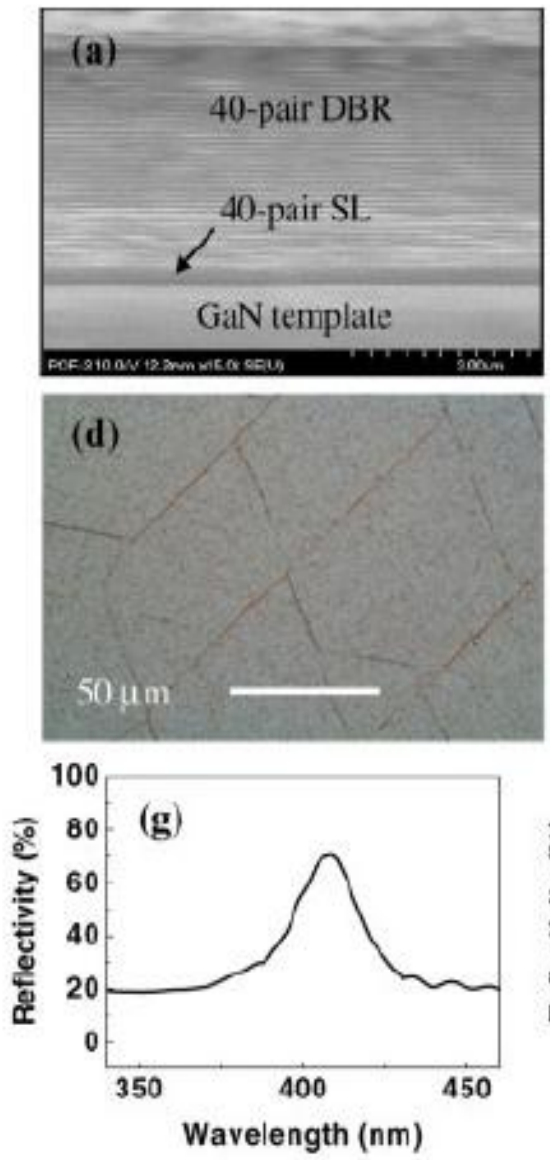

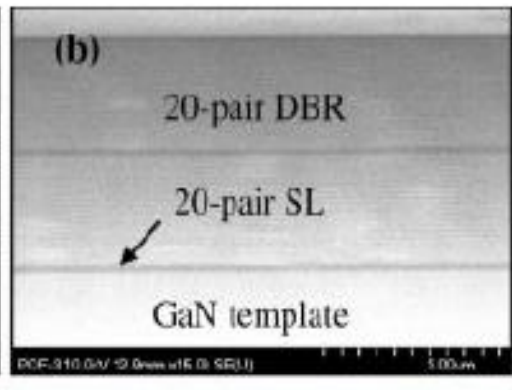

(e)

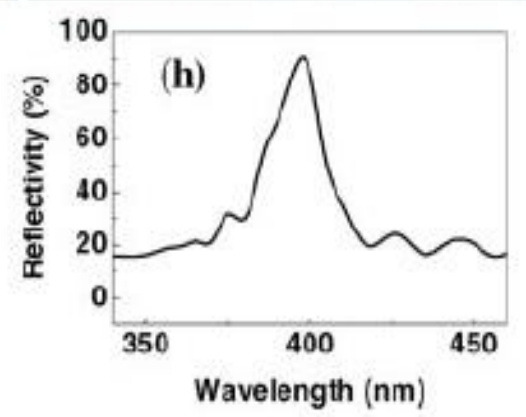

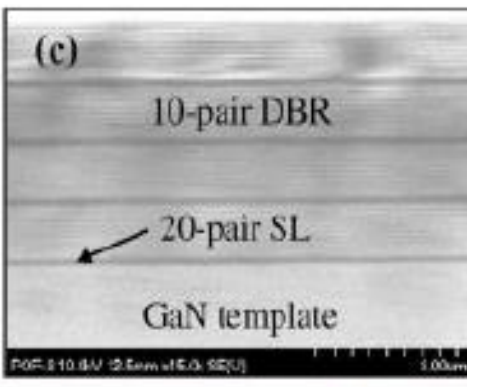
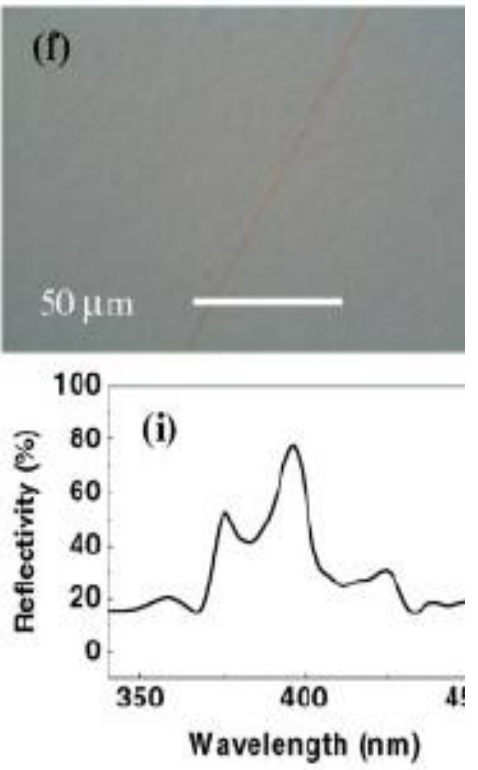

Figure 2-24 - Cross sectional SEM images, optical microscope images, and reflectivity measurements for 40 period $A l_{0.35} G a_{0.65} N / G a N$ DBRs. From Ref. 78. 


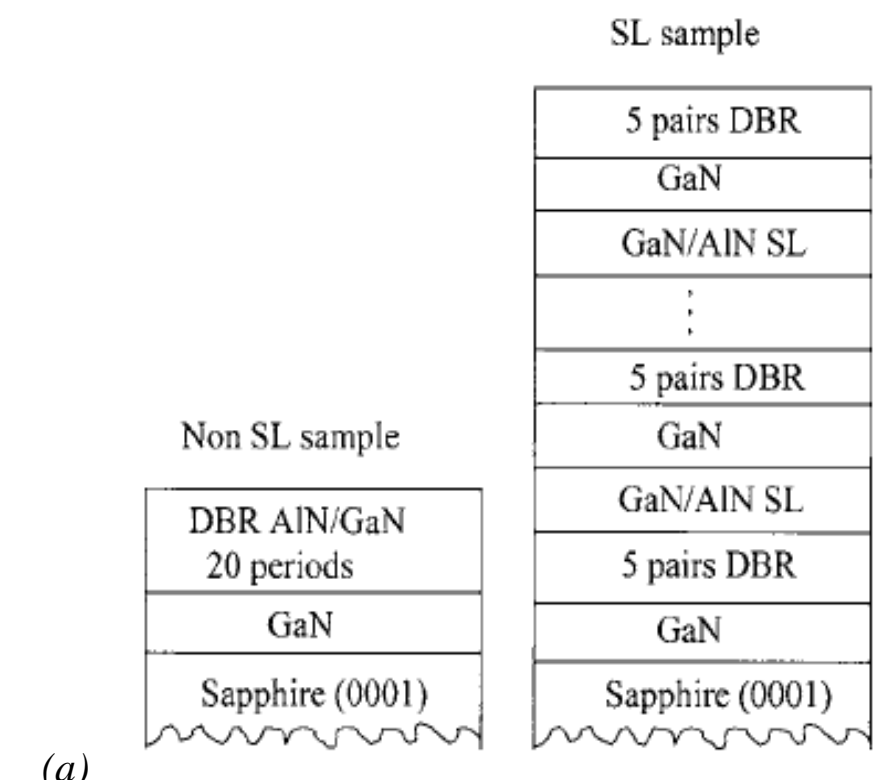

(a)

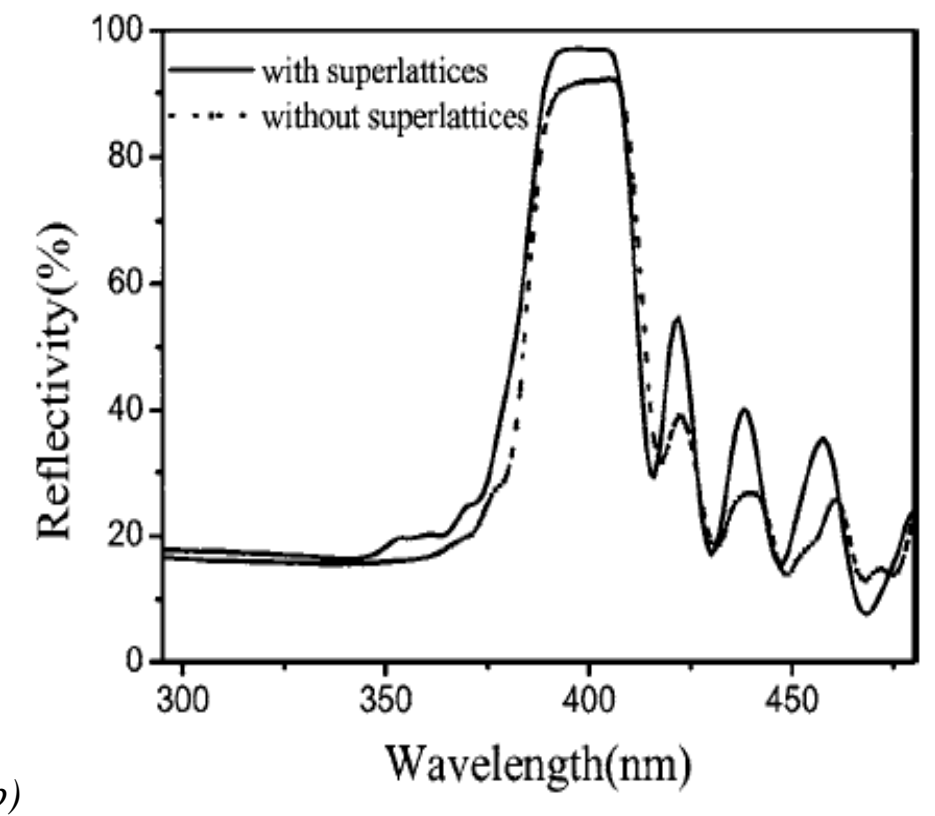

Figure 2-25 - (a) Schematic and (b) reflectivity response of 20 pair AlN /GaN DBR with and without 5.5 periods of AlN/GaN superlattice insertion layers. From ref. 76. 


\section{CHA P T E $3-$ EX PERIMENTAL M T H O D}

\subsection{Introduction}

This work has investigated the development and characterization of III-Nitride films and related devices via MOVPE. In this chapter, the equipment used for material growth and characterization is described. $\mathrm{XRD}$, transmission, reflectivity, ellipsometry and Hall measurements were employed to extract critical material properties such as lattice parameters, band gap, refractive index, and carrier concentrations. Each technique is described in this chapter.

\subsection{MOVPE System}

All growths in this work have been conducted using an Aixtron 200/4 RF-S MOVPE system shown in Figure 3-1. This particular system contains a horizontal reactor with 2" wafer growth capabilities. The horizontal design of the reactor is used to achieve laminar flow allowing for abrupt changes in growth interfaces. The flow chart shown in Figure 3-2 illustrates the various components of the MOVPE system. The main growth takes place in the reactor; however, as illustrated in Figure 3-2, there are a number of different elements which support the process.

The system is controlled remotely with a software program called CACE. All instructions and recipes for the MOVPE are initiated from the software which interfaces with the different components of the system through the electronics rack. The Gas Mixing System (GMS) is a network designed to mix and deliver gasses to the reactor and is shown in Figure 3-3. As timing and composition of gas determines epilayer quality, the gas mixing system is a critical component of the MOVPE system. The primary elements of the GMS include pneumatically controlled valves, pressure controllers, mass flow controllers, group III and V sources, and dopant sources. The vacuum level for this system ranges from 0 to 1000 mbar and is sustained with a rotary vane pump. The glove box is attached to the reactor so that it opens into nitrogen atmosphere to ensure a clean environment for loading and unloading samples as well and preventing oxygen contamination of the growth chamber. The reactor is the stainless steel chamber in which the growth process occurs. Being a horizontal reactor, gas enters at one end of the reactor, flows horizontally through the chamber, and is exhausted through the opposite end as 


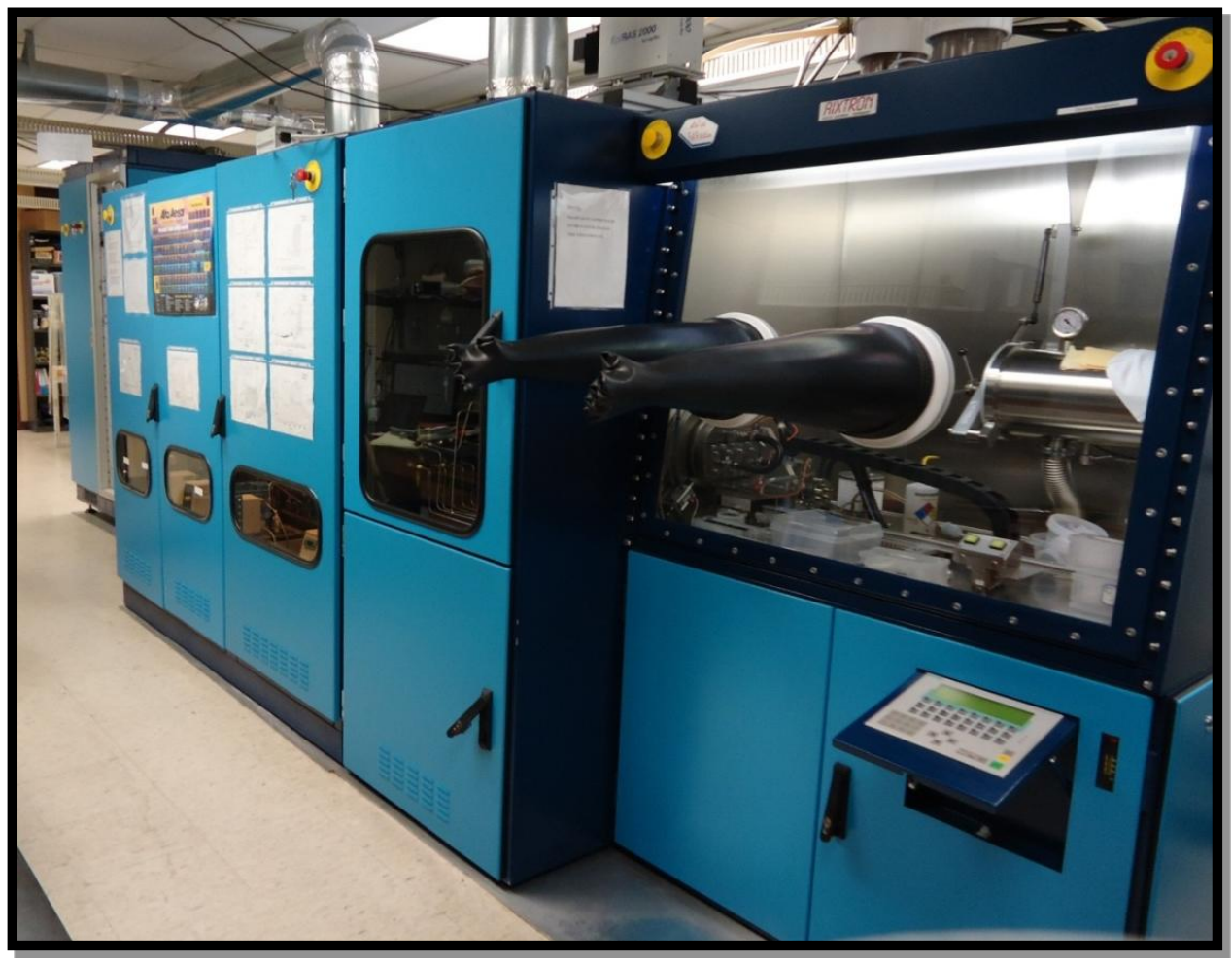

Figure 3-1 - Aixtron 200/4 RF-S MOVPE System.

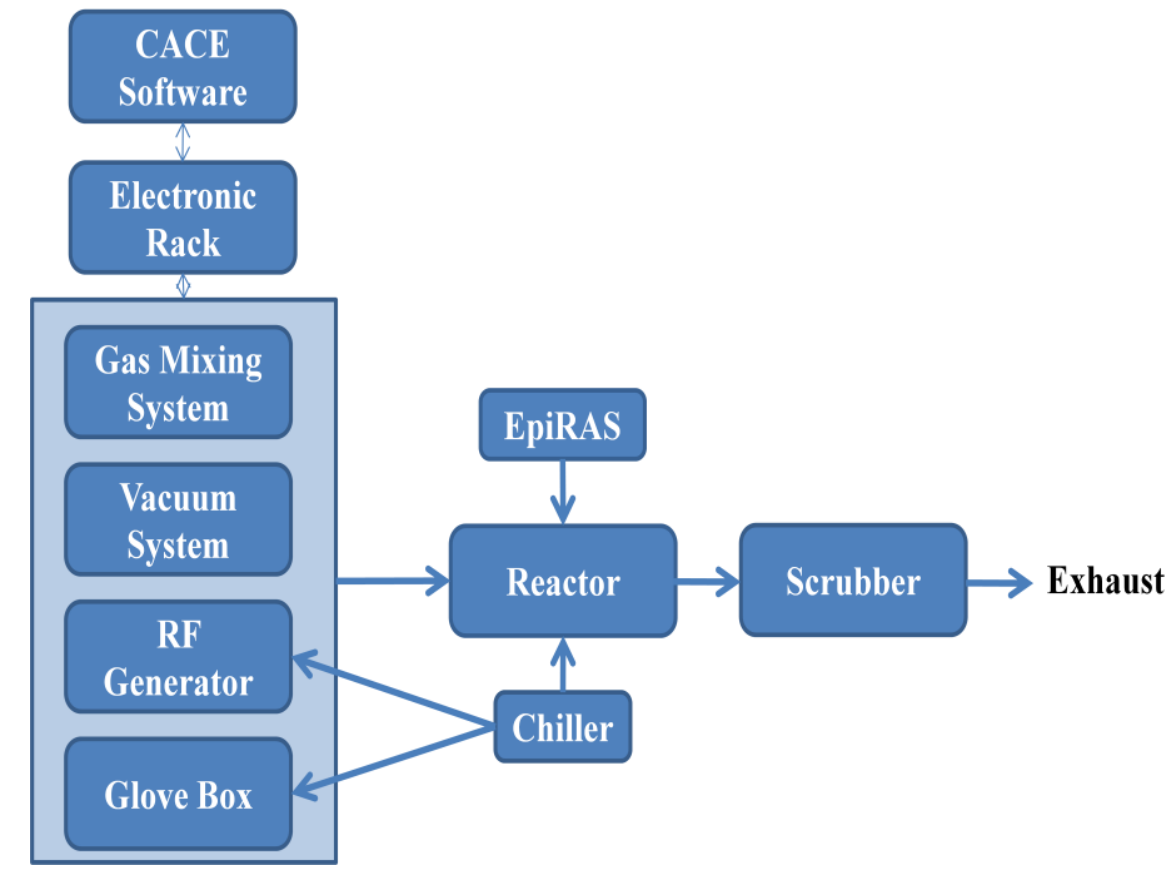

Figure 3-2-MOVPE System Components 


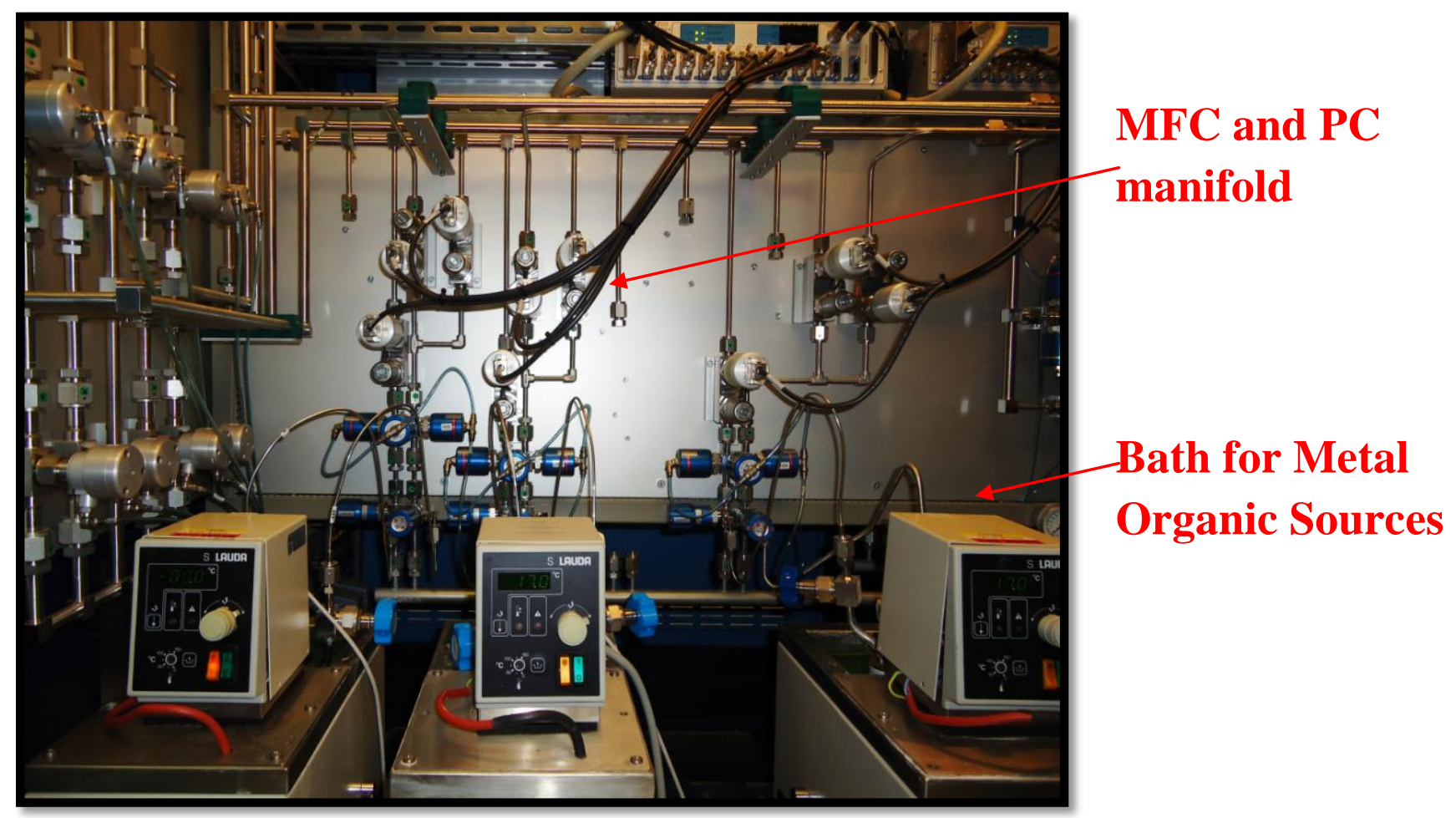

Figure 3-3-Gas mixing cabinet.

illustrated in Figure 3-4. The wafer is located on a silicon carbide coated graphite disc, known as the susceptor, which is rotated during growth to ensure uniform heating and gas distribution over the wafer. The metalorganic and ammonia gas supply are separated by quartz plate in order to avoid parasitic reaction until just before the growth site. The susceptor is heated to the growth temperature $\left(600{ }^{\circ} \mathrm{C}-1100{ }^{\circ} \mathrm{C}\right)$ via induction heating. The coil below the susceptor is excited with a radio frequency $(\mathrm{RF})$ current. The alternating current in the coil creates an alternating magnetic field which induces eddy currents in the sucesptor. The resistance of the susceptor leads to $I^{2} R$ heating. The system is cooled with a closed loop water chiller which circulates distilled water at $16-19{ }^{\circ} \mathrm{C}$ to the Reactor, Glove Box, and RF Generator. After passing through the reactor, the process gas is directed to the AIX-TOX scrubber unit. A $30 \%$ solution of sulfuric acid and DI water is showered over exhausted gas to decompose the ammonia and excess metal organics. 


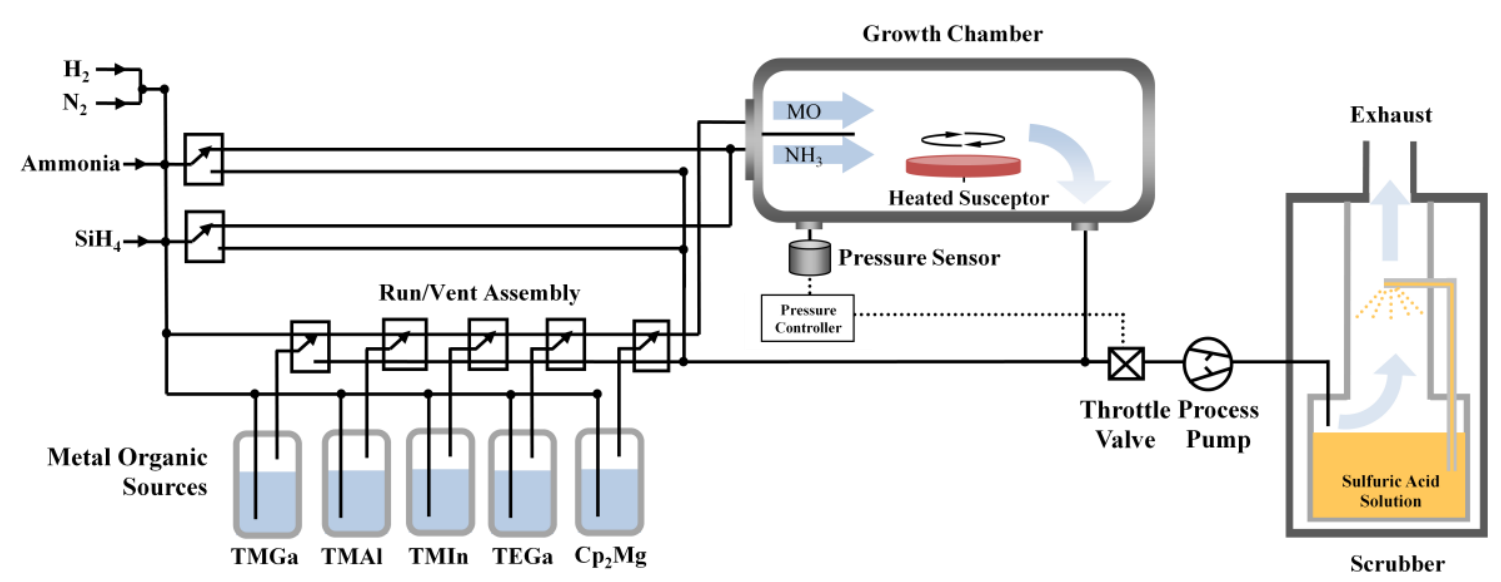

Figure 3-4 - Illustration of AIXTRON MOVPE system.

\subsection{Temperature Calibration}

Thin film growth is heavily dependent on the substrate temperature during growth ${ }^{26}$. As a result, special attention must be paid to the calibration of the temperature to adjust for any drift in the equipment. The MOVPE is equipped with a light pipe for temperature readings. The light pipe measures the radiation from the heated susceptor and converts it to a temperature based on sensitivity parameters set in the Luxtron Temperature Controller. In order to properly set the sensitivity factors for the light pipe, a secondary means of measuring the susceptor temperature must be used. In this work, an Omega OS-3000 AS infared (IR) pyrometer has been used. This particular pyrometer has a detector sensitive in the $2.1 \mu \mathrm{m}-2.3 \mu \mathrm{m}$ spectral range. The quartz ceiling in the reactor was removed to open a clear path from the top viewport of the reactor to the susceptor. A clean p-type silicon (100) was loaded on the susceptor as the reference surface since the emissivity of silicon is well known and is almost constant over the temperature range of interest $^{86}, 600^{\circ} \mathrm{C}$ to $1000^{\circ} \mathrm{C}$. The gas flows into the reactor were set to similar conditions used for GaN growth. The surface temperature was of the silicon wafer was measured with the pyrometer and compared to the setpoint. Figure 3-5 shows the discrepancy between the setpoint and the measured temperature before calibration. A second measurement was performed with a fiber optic pyrometer to repeat the measurement with the quartz ceiling in place. The fiber optic pyrometer collects the radiation over a smaller area and therefore can be used to measure the temperature with the ceiling in the reactor as long as the pyrometer is properly aligned over the hole in the ceiling. As shown in the graph, the temperature measured with the ceiling was 
approximately the same as that measured without the ceiling. Therefore, all subsequent pyrometer tests were performed without the ceiling using the IR pyrometer gun. Following the instructions outlined in the Appendix A, the sensitivity factors were adjusted until the setpoint was the same as the value measured with the pyrometer as shown in Figure 3-5. At the time of this writing, there were two susceptors that could be used with the MOVPE system and both were tested after the temperature calibration was performed. As shown in this graph, both have similar temperature characteristics. A pyrometer test, as outlined in Appendix B, is done periodically to verify the integrity of the system and also after any maintenance on the RF coil.

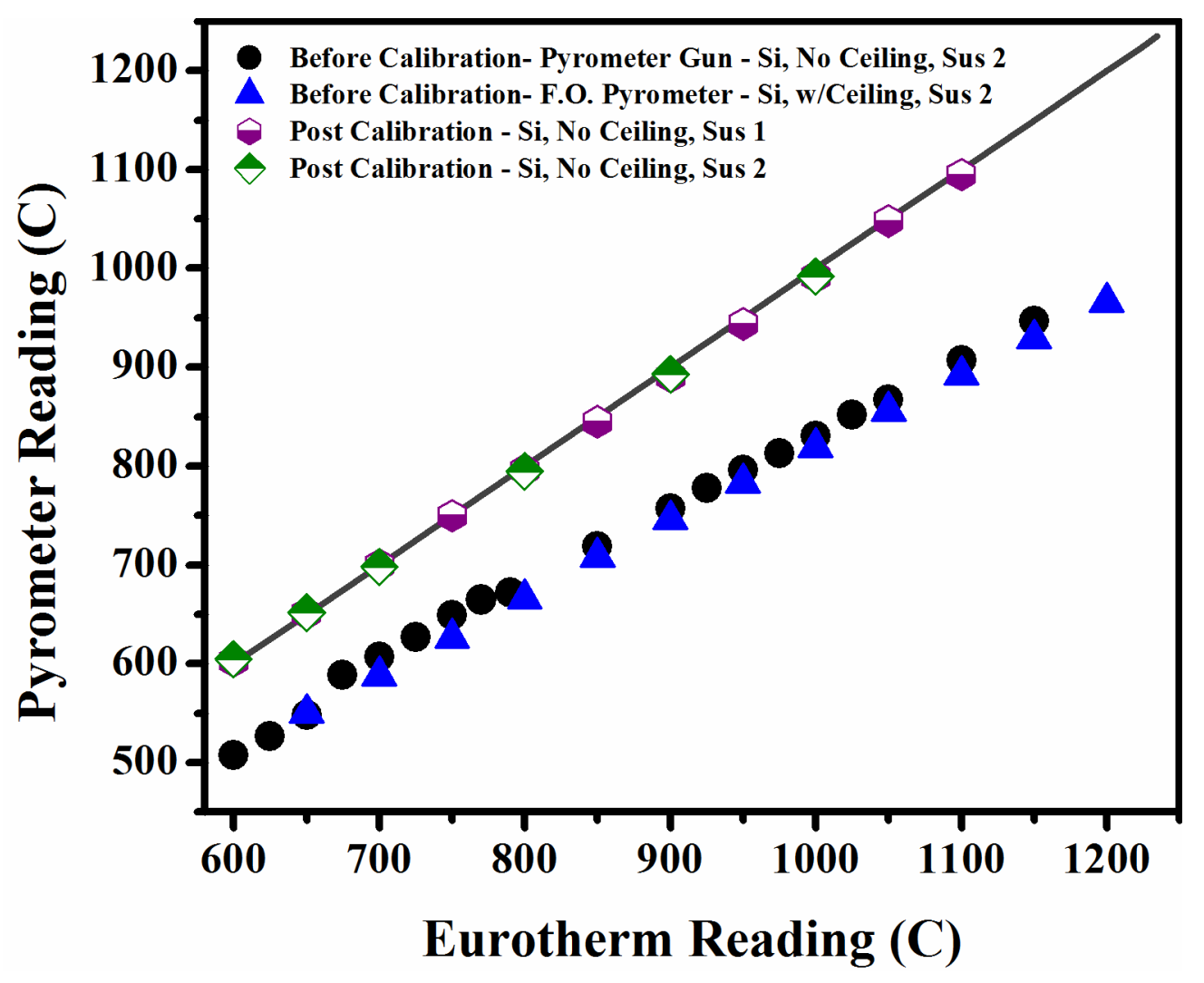

Figure 3-5 - Measured substrate temperature and setpoint before and after calibration. Eurotherm reading corresponds to the setpoint entered in the CACE software. 


\subsection{Growth Rate}

Knowing the exact growth rate of the materials is critical to both device fabrication and also system maintenance. Essentially, all growth parameters influence the growth rate. In this section, the influence of the temperature, source supply, and reactor pressure will be discussed. In the MOVPE system, the EpiRAS is an in situ monitoring system allows for real time analysis of growth rate and surface quality. This system utilizes normal incidence reflectometry at a single wavelength. Light is directed approximately normal to the surface of the sample through the reactor window and the reflection is measured with a photodetector. The wavelength is chosen such that it is not absorbed by the material of interest. In the case of a $\mathrm{GaN}$ epilayer grown on a sapphire substrate, as illustrated in Figure 3-6, the reflection from the surface undergoes a $180^{\circ}$ phase shift since the refractive index of GaN, $n=2.35$, is greater than that of the ambient gas conditions of the reactor. In contrast, the reflections from the interface between the GaN layer and sapphire substrate do not have an associated phase shift since the refractive index of sapphire, $n=1.78$, is less than that of GaN. Reflections within the sapphire are negligible in this case since the backside of the sapphire wafer is rough and scatters the light. The reflections from the surface of the GaN layer and also from the interface between the $\mathrm{GaN}$ and sapphire undergo constructive or destructive interference depending on thickness of the GaN layer. In order for the reflections 1 and 2 shown in Figure 3-6, to undergo constructive interference, the rays traveling within the GaN layer must travel an odd number of

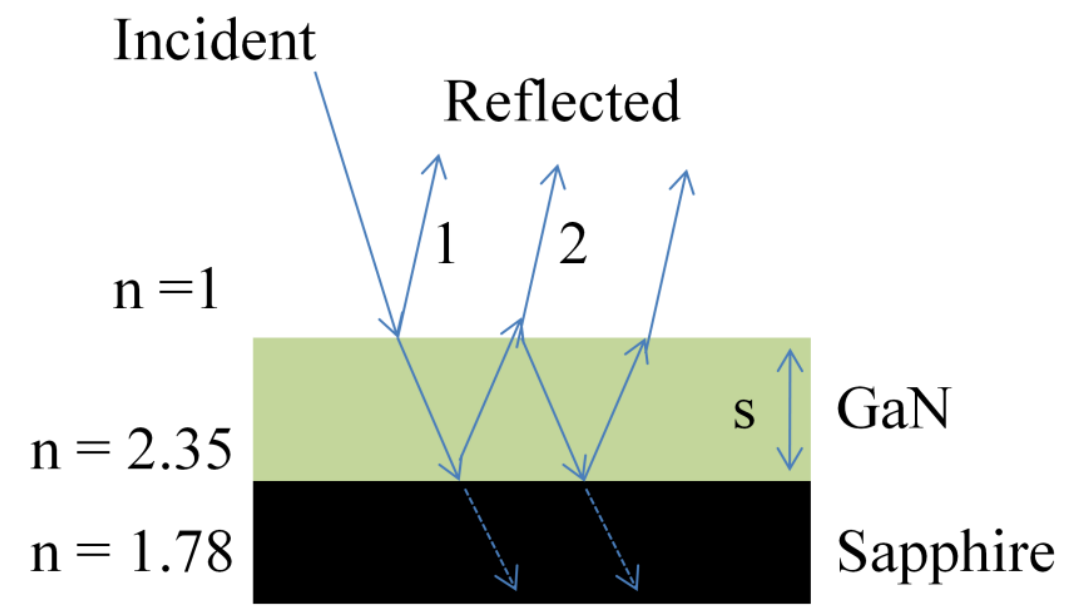

Figure 3-6-Illustration of ray paths in GaN film. 
half wavelengths (i.e. $2 s=\frac{2 m-1}{2} \lambda$ where $m$ is an integer, $2 s$ is the distance traveled by the ray, and $\lambda$ is the wavelength within the material). Figure 3-7 shows the oscillations during growth of $\mathrm{GaN}$ as measured with the EpiRAS. The growth rate can be derived from the oscillation period as follows:

$$
\frac{d s}{d t}=\frac{\lambda_{o}}{2 n T}
$$

where $s$ is the layer thickness, $T$ is the oscillation period, $n$ is the refractive index of the material, and $\lambda_{\mathrm{o}}$ is the incident wavelength. Furthermore, the amplitude of the oscillations seen in Figure 3-7 give insight into the surface quality of the film. A significant decrease in the amplitude indicates roughening of the surface. Whereas a slight decrease may only indicate non-uniformity over the spot sight of the light. The reflectivity in this figure was measured with $650 \mathrm{~nm}$ incident light and includes the desorption and AlN nucleation steps as well as the GaN main layer. The periodicity is approximately 282 seconds indicating a growth rate of approximately $29 \mathrm{~nm} / \mathrm{min}$ or $1750 \mathrm{~nm} / \mathrm{hr}$.

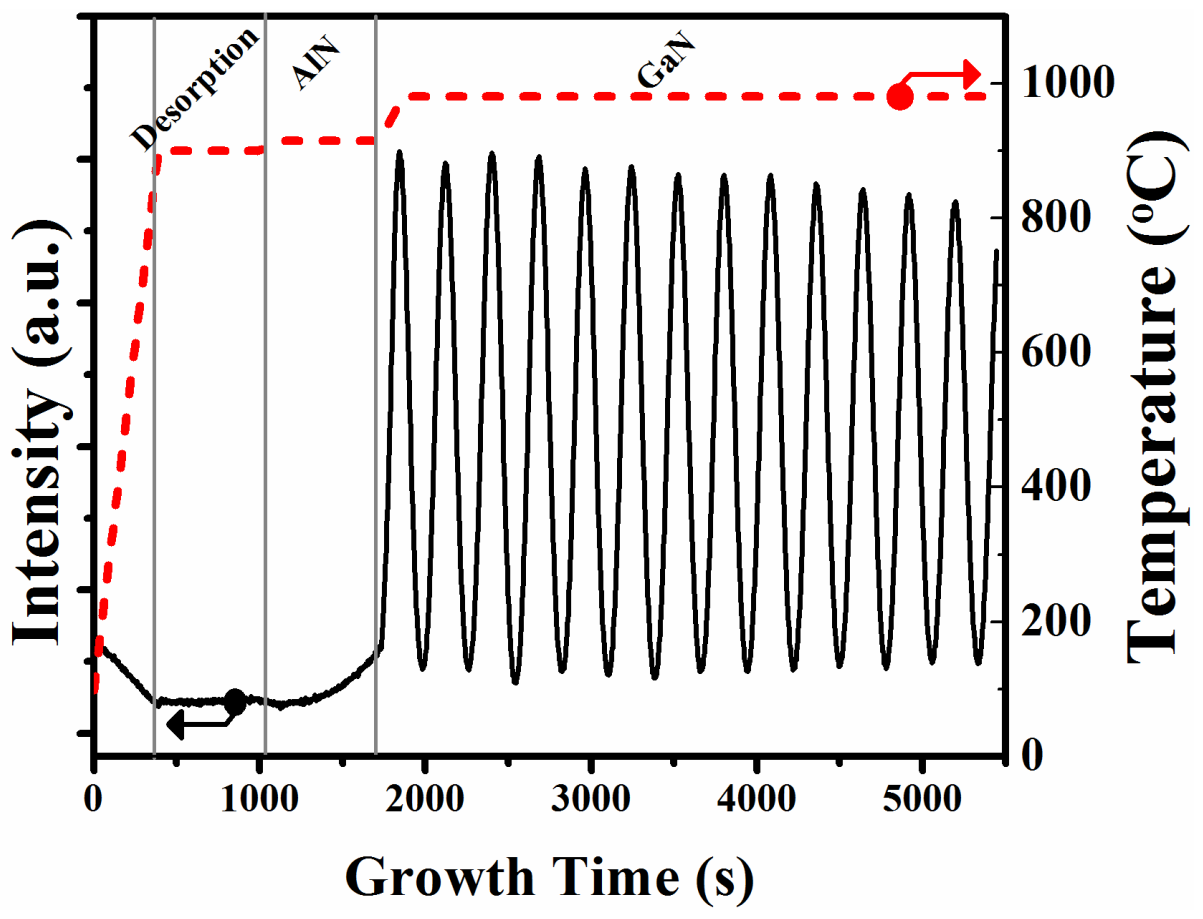

Figure 3-7-EpiRas oscillations during GaN growth on sapphire. 
As already mentioned, growth can occur in two regions: kinetically limited or transport limited. To achieve the maximum growth efficiency, it is ideal to operate in the mass transport limited regime in which there is little dependence of the growth rate on the temperature. Therefore, the growth rate has been investigated as a function of the temperature to ensure we are growing in the proper window. Using the periodicity of the EpiRAS oscillations to determine the growth rate, the growth temperature was varied by approximately $100{ }^{\circ} \mathrm{C}$ during GaN growth. The results are shown in Figure 3-8. At low temperatures, the growth is kinetically limited and the growth rate increases with increasing temperature. Over the midtemperature region, the growth rate is almost independent of growth temperature. Finally, at high growth temperatures there is a subtle decrease in the growth rate due to desorption of species from the surface. For this work, temperatures in the mass transport limited regime were targeted for GaN growth. It is necessary to periodically verify the growth rate, especially after servicing the reactor.

Within the mass transport limited regime, the growth rate is expected to vary linearly

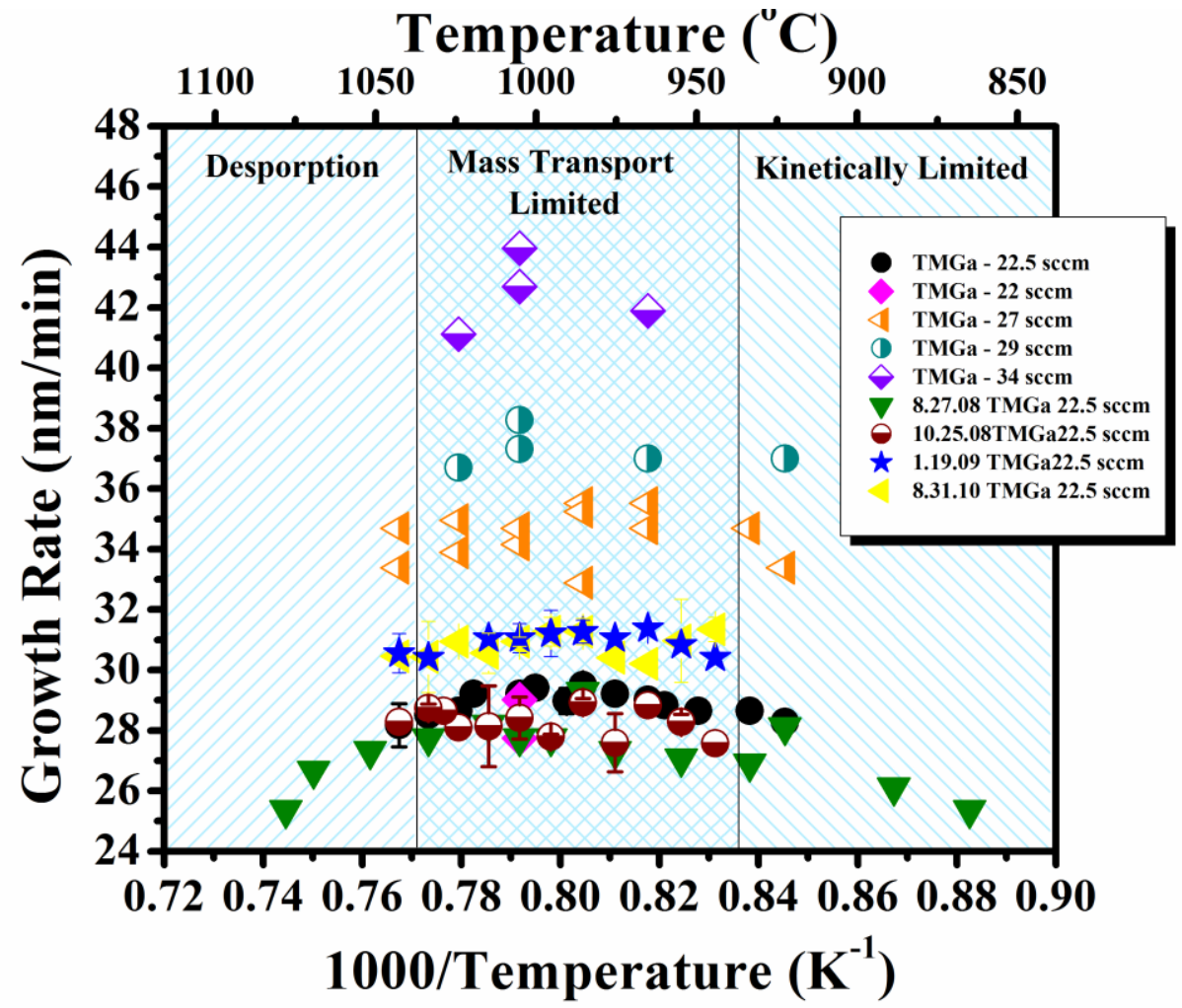

Figure 3-8 - Growth rate of GaN at different temperatures for various TMGa flow rates. 
with the group-III source since the growth rate defined in Eq. 2-1 depends on the concentration gradient of the group-III species from the inlet to the growth surface. However, this is only true when all the group-III elements at the surface are being incorporated into the growth. Figure 3-9 summarizes the GaN growth rate as a function of the Ga supply. As expected, we observe a linear dependence of the growth rate on the TMGa flow. Therefore, a TMGa flow rate was chosen that results in good surface morphology as well as electrical properties. In this figure, there is an observable shift in the growth rate as measured across two years. This is due to changes in the reactor over time and also the use of new quartz pieces in the chamber.

The relationship between the group- $\mathrm{V}$ precursor and the growth rate is the opposite as that for the group-III precursor. Figure 3-10 shows the dependence of the GaN growth rate on the ammonia source and push flow rates. As mentioned previously, the pyrolysis of ammonia is inefficient; therefore the ammonia supply is always supersaturated. Under this condition, increasing the supply does not increase the growth rate as with the group-III supply. However, it

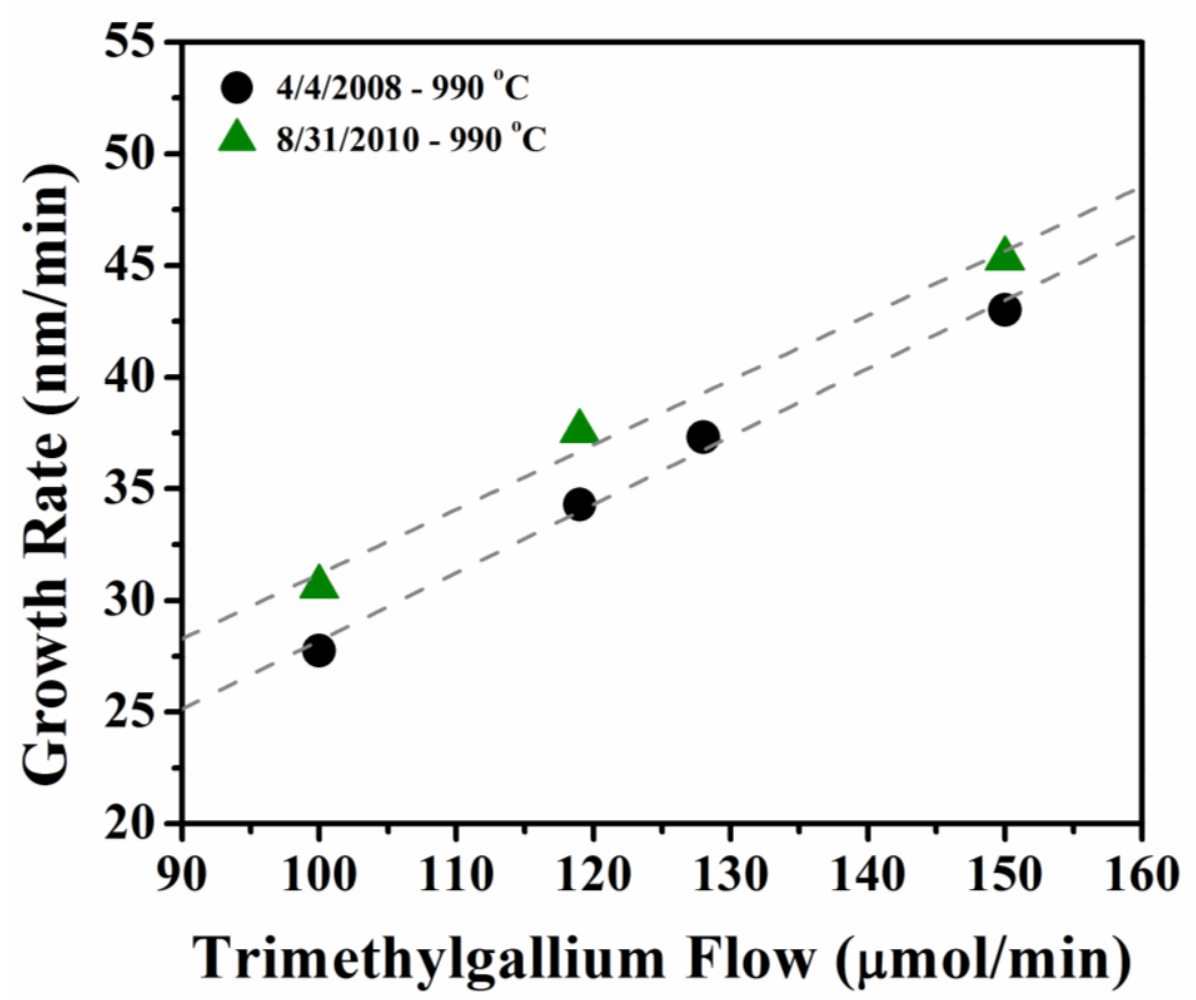

Figure 3-9 - Growth rate of GaN under various TMGa flow rates. Ammonia source was fixed at 1500 sccm and temperature was $990^{\circ} \mathrm{C}$.

does 

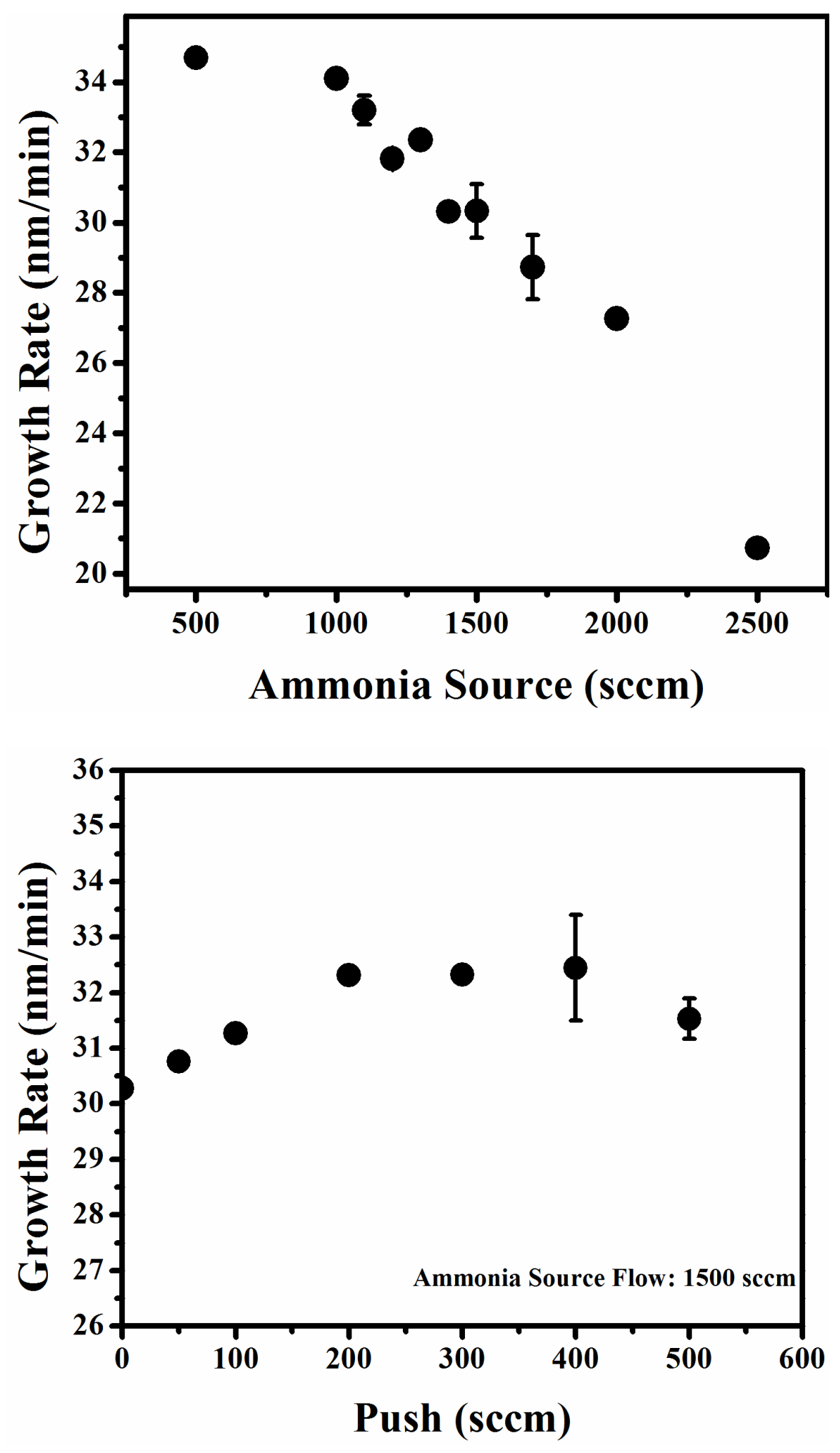

Figure 3-10 - Growth rate of GaN at different (a) ammonia source flows and (b) ammonia push flows. TMGa flow rate was $22.5 \mathrm{sccm}$ and the temperature was at $990^{\circ} \mathrm{C}$. 
increase the parasitic reactions because the metal organic source and this results in a decrease of the growth rate.

In addition to substrate temperature and source supply, the reactor pressure is also an important process parameter. The MOVPE system used in this work is a low pressure system and typically growths are performed at 200 mbar or lower. The low pressure is not necessarily required for MOVPE growth, however it does reduce the parasitic reactions between the precursors. In general, the lower the reactor pressure, the fewer collisions between the atoms in the gas phase. Figure 3-11 shows how the growth rate increases with decreasing pressure.

\subsection{Mass Flow Controller Verification}

The mass flow controllers (MFCs) are a critical component of the MOVPE system as they control the delivery of the source materials. In order to design and grow accurate structures

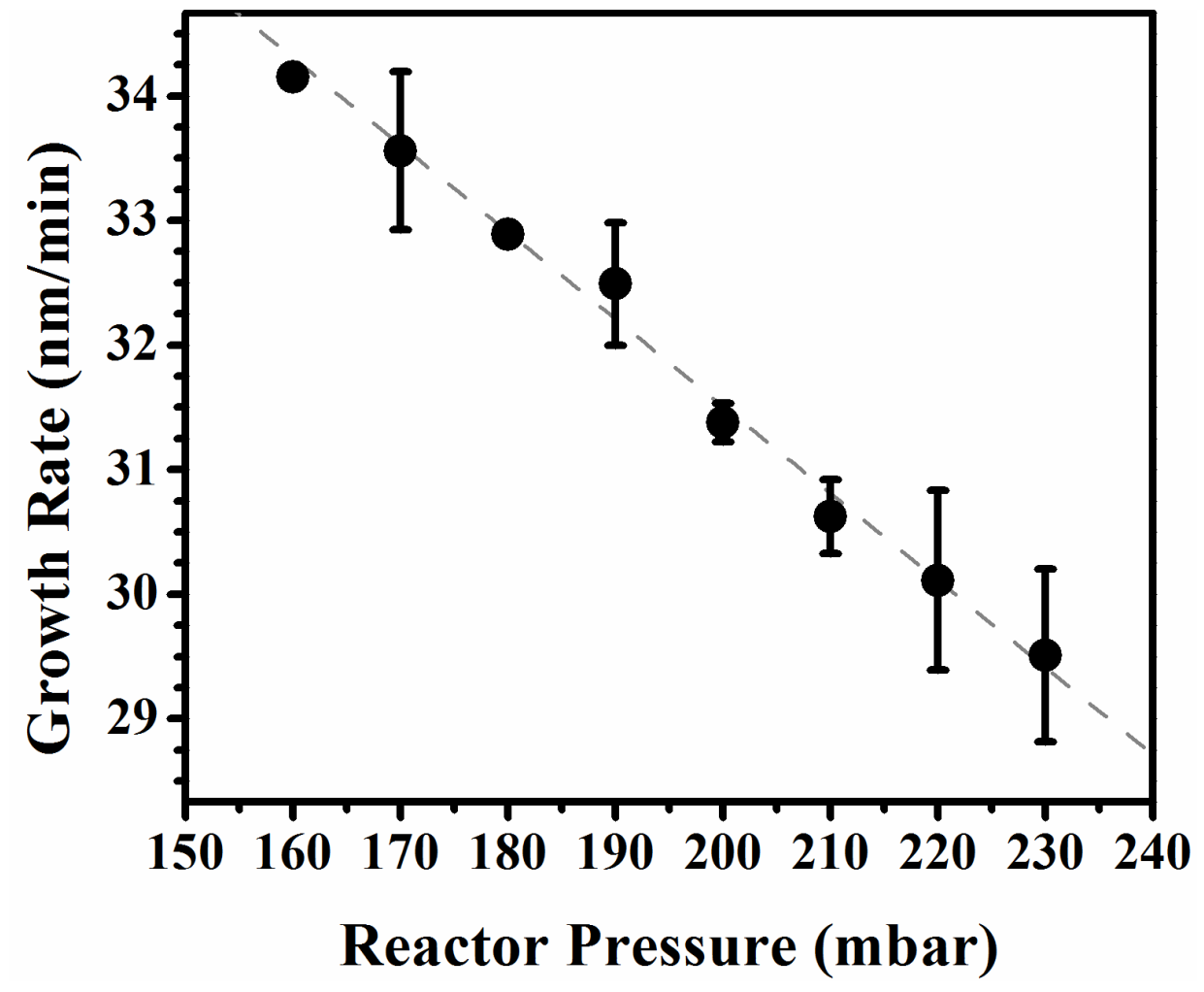

Figure 3-11 - Relationship between the reactor pressure and GaN growth rate. Ammonia source was $1500 \mathrm{sccm}, \mathrm{TMG}$ a source was $22.5 \mathrm{sccm}$, and reactor temperature was $990^{\circ} \mathrm{C}$. 
with abrupt interfaces, it is imperative that the MFCs be operating properly. To verify proper operation, rate of fill tests should be periodically performed. The procedure is outlined in Appendix $\mathrm{C}$, however, in general the reactor is pumped down to the base pressure and then refilled with nitrogen or hydrogen through various MFCs. The rate at which the reactor refills is timed. The fill time between multiple MFCs are compared in order to verify the operation. Figure 3-12 illustrates that the fill time for different gas flows is the same between the ammonia MFC and the Run-Hydride MFC. This indicates that the two MFC are supplying the specified amount of gas.

Performing routine maintenance and verifying the results of the tests described in this section are critical to maintaining the system. In order to achieve growths and devices that are repeatable, it is important to verify that the equipment is working properly and also consistently. Small adjustments must be made over time to correct drift in these measurements. In addition to verifying the equipment operation, the material must also be characterized. Common techniques used in this work to measure material properties include XRD, transmission, reflectivity, and also Hall measurements.

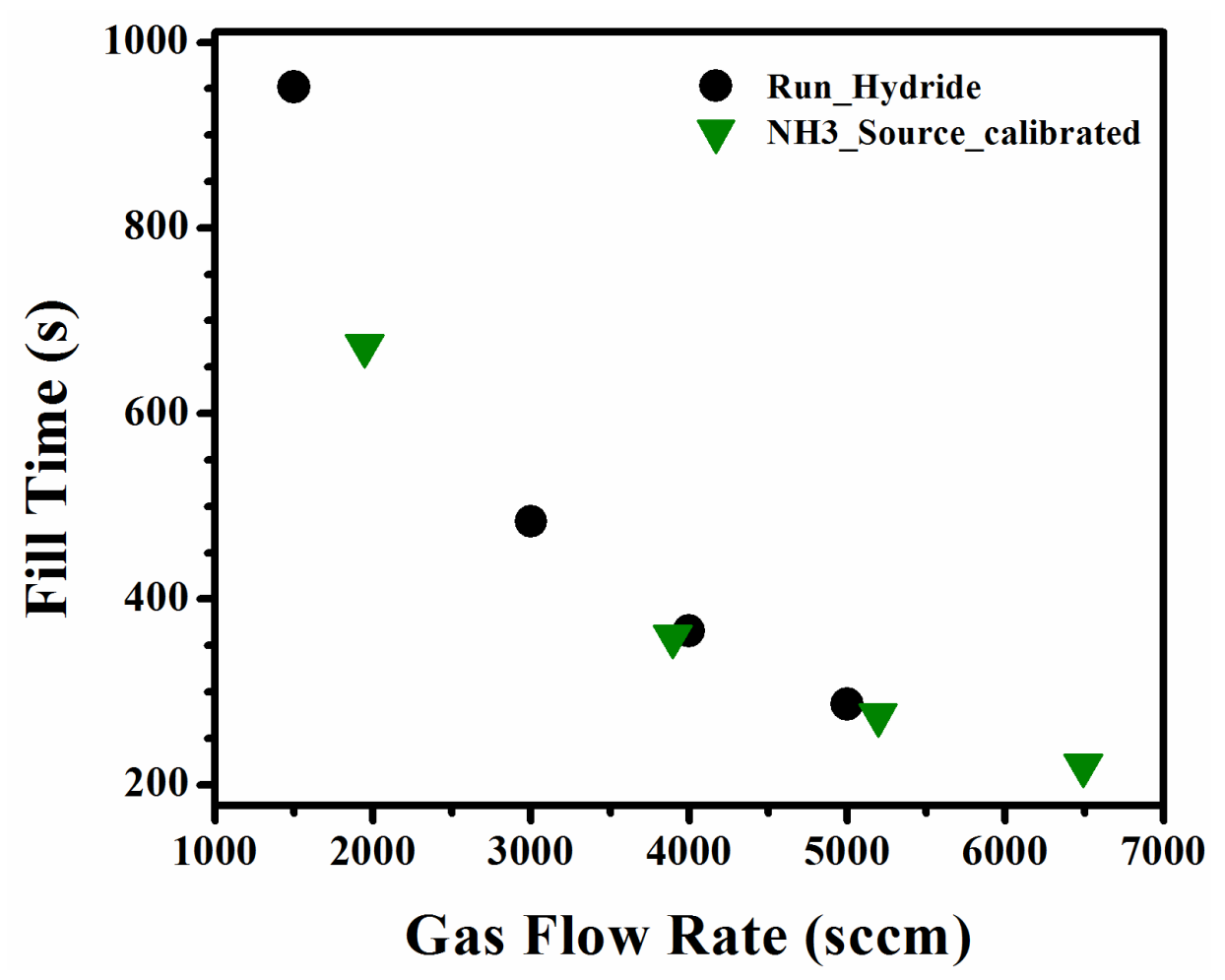

Figure 3-12 - Reactor fill time for different MFCs. 


\subsection{X-Ray Diffraction}

$\mathrm{X}$-Ray Diffraction (XRD) is a non-destructive characterization technique that provides important information regarding the structural properties of the materials. This includes both the $a$ - and $c$-lattice parameters. These properties allow for the verification of the materials grown, calculation of alloy concentration, and measurement of the strain. In this work, all high resolution XRD measurements were taken with a Bruker D-8 Discover four circle diffractometer equipped with a 4 bounce symmetric Ge (022) monochromator. A Goebel mirror was used to collimate the beam prior to the monochromator. Figure 3-13 shows the principle of X-Ray Diffraction $^{87}$. X-rays incident on a crystal are diffracted from the individual atoms in the lattice. Constructive interference occurs when distance $\mathrm{AB}$ in this figure is equal to $2 d$, in which $d$ is the lattice plane spacing. Under this condition, Bragg's law is satisfied and the relationship between the place spacing $(d)$ and the angle of incident $\mathrm{x}$-rays $(\theta)$ is given by ${ }^{87}$ :

$$
n \lambda=2 d \sin (\theta)
$$

In which $n$ is the order of diffraction, $\lambda$ is the wavelength of the $\mathrm{x}$-ray radiation, $d$ is the lattice spacing, and $\theta$ is the angle between the incident $x$-ray beam and the sample surface. Usually, the

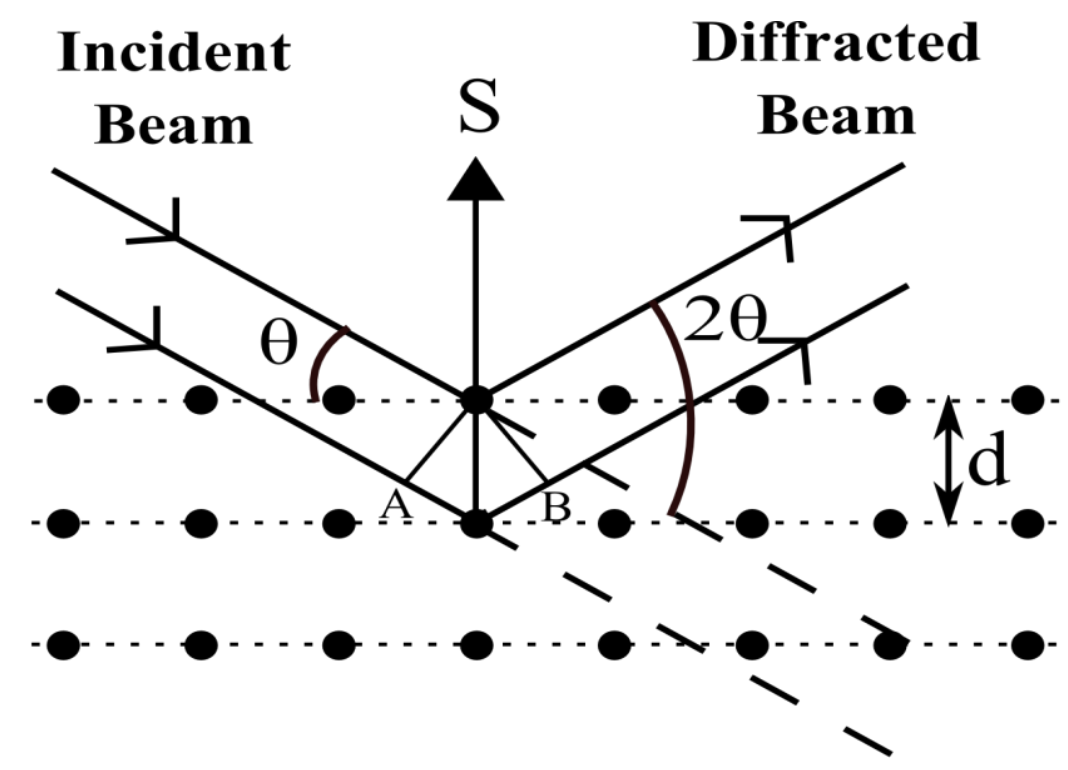

Figure 3-13 - Illustration of X-Ray Diffraction. Modified from Ref. 87. 
experimental setup measures $2 \theta$ and is therefore represented as the $\mathrm{x}$-axis in $2 \theta-\omega$ scans. These scans are typically used to determine the out-of-plane lattice parameter and also superlattice periodicity. The presence of satellite peaks in $2 \theta-\omega$ scans verifies the structural integrity of superlattices as these are only present if there are discrete layers in the structure.

Reciprocal space mapping is another XRD technique which is used to extract information related to the strain and also the in-plane lattice parameter ${ }^{87}$. Reciprocal space mappings are the 20- $\omega$ scans collected around a reciprocal space point. Figure 3-14 illustrates the relationship between the lattice spacing and the reciprocal lattice space. Above the sample surface is a mapping of the reciprocal space lattice. The scattering vector $S_{h k i l}$ is equal to $k_{h}-k_{o}$. Diffraction occurs when the scattering vector ends at a reciprocal space point. The magnitude of $\mathrm{k}_{\mathrm{h}}$ and $\mathrm{k}_{\mathrm{o}}$ is $1 / \lambda$ in which $\lambda$ is the wavelength of the $x$-ray source. The orientation of vector $S$ is changed by modifying the angle with which the incident rays meet the sample and also the position of $2 \theta$.

In this work, GaN and related hetero-structures have been investigated. Standard $2 \theta-\omega$ scans will be used to extract the lattice parameter and verify the growth conditions for the films. Periodically, samples are measured to verify the operation of the MOCVD but also to evaluate the growth conditions developed. This work has focused on superlattices development and will be discussed in subsequent sections. By evaluating the lattice parameter of both the superlattice and also the buffer layer, the strain state of the material can be determined. In relatively symmetric superlattices, the strain is distributed in both the AlN and GaN layers ${ }^{73}$. This strain profile may be considerably different than what accumulates in a random alloy. It is anticipated that the use of the superlattice will allow for higher content alloys to be achieved before the onset of cracking. Reciprocal space mapping also allows for the coherent or incoherent nature of the film to be determined. In coherent films, the lattice parameter of the top layer is dictated by the lattice underneath. Figure 3-15 illustrates this concept ${ }^{87}$. The peak labeled L refers to the $\mathrm{Al}_{x} \mathrm{Ga}_{1-x} \mathrm{~N}$ layer grown on a GaN substrate labeled $\mathrm{S}$. The vertical alignment of the substrate and the layer in Figure 3-15(a) indicates coherent growth. In this regard, the $\mathrm{Al}_{x} \mathrm{Ga}_{1-x} \mathrm{~N}$ layer has the same in-plane lattice parameter as the underlying GaN substrate. Figure 3-15(b) show a relaxed $\mathrm{Al}_{x} \mathrm{Ga}_{1-x} \mathrm{~N}$ layer which does not have the same lattice parameter as the substrate. The relaxation of films occurs in various ways but commonly include large scale cracking or defect formation. 


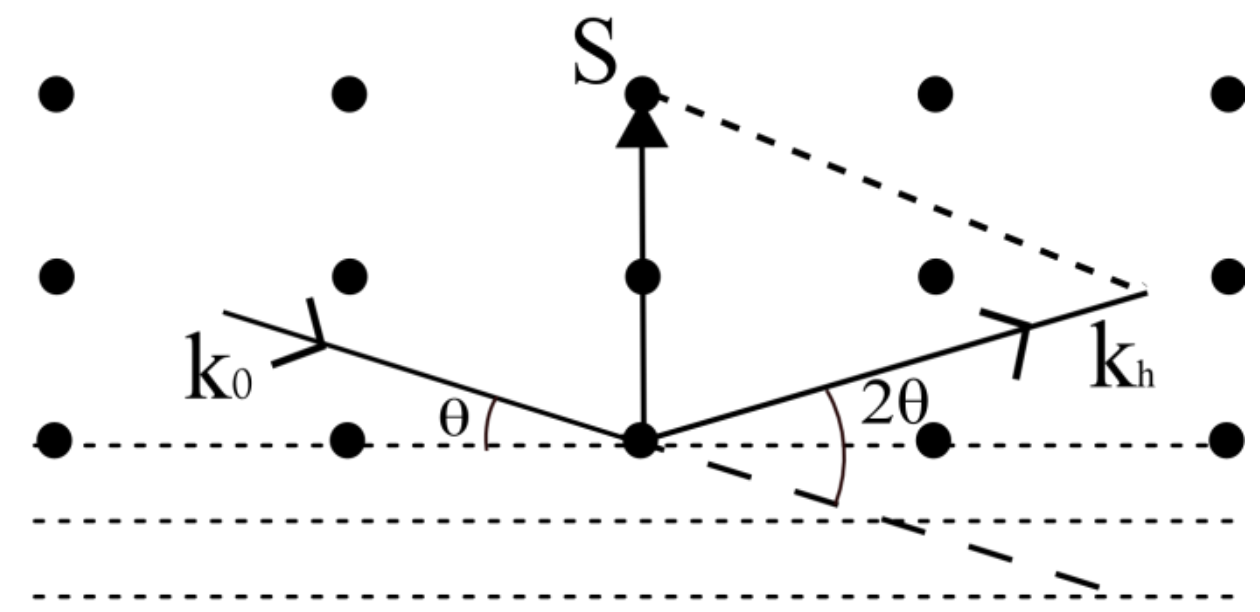

Figure 3-14 - Schematic showing relationship between real lattice parameters and reciprocal lattice space. Modified from Ref. 87.
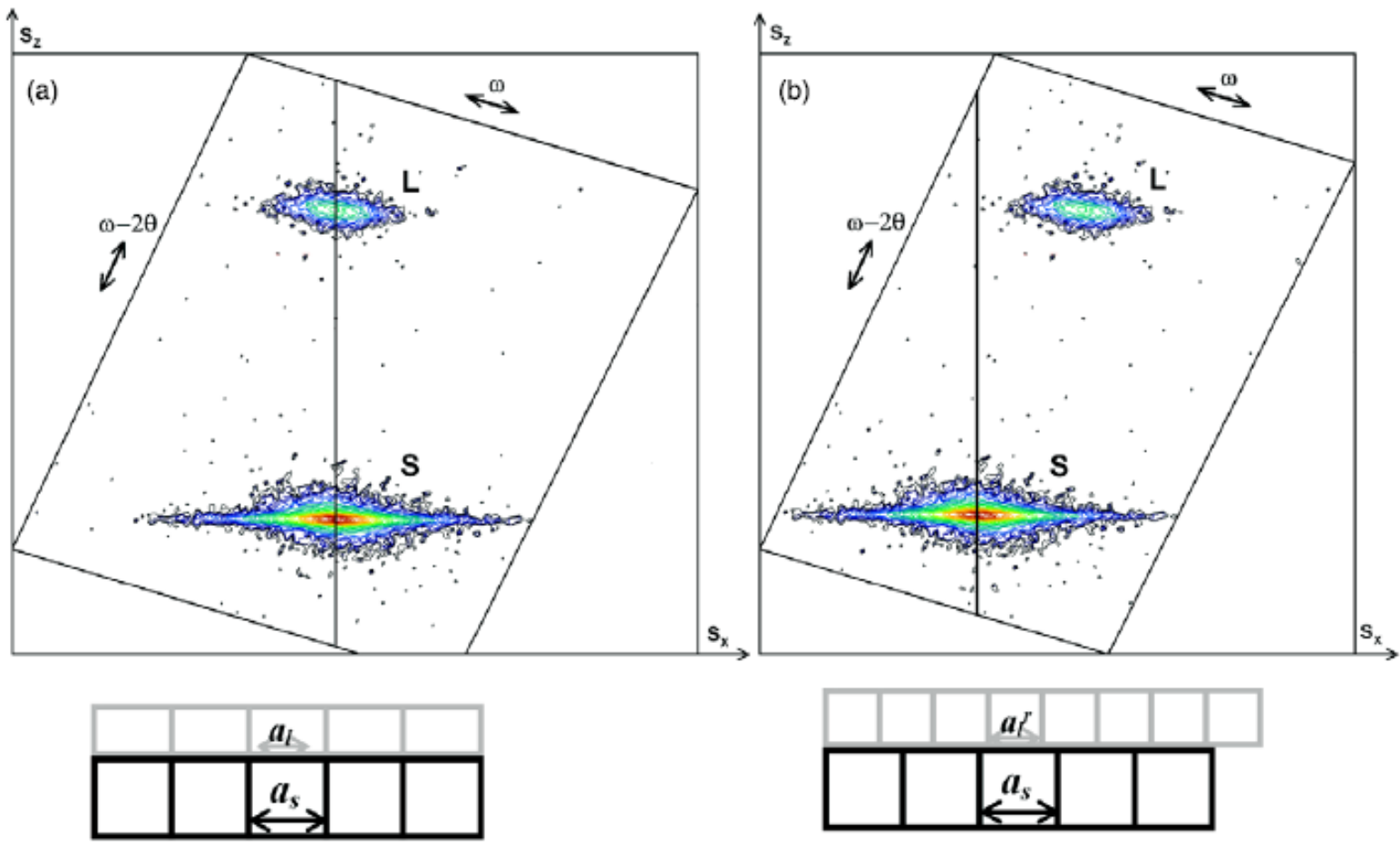

Figure 3-15 - Example reciprocal space mapping of a (a) strained and (b) relaxed $A l_{x} G a_{1-x} N$ layers on GaN. From Ref. 87. 


\subsection{Transmission Measurements}

For the characterization of thin films and Distributed Bragg Reflectors in particular, transmission and reflectivity measurements are used. Transmission measurements provide an accurate means of determining the film thickness, or if the film thickness is well know, it can be used to find the refractive index. Transmission measurements require a white light source and a spectrometer. In this work, measurements have been taken with a halogen/deuterium white light source and a BW-Tek CCD spectrometer as shown in Figure 3-16. The setup can also be constructed with a white light source, a monochromator, and a photodetector. Figure 3-17 shows the transmission measurement of a $\mathrm{GaN}$ film on a sapphire substrate. This measurement was obtained by measuring the transmission of light through the GaN sample and dividing by the transmission through a bare sapphire wafer. For wavelengths below the bandgap $(<364 \mathrm{~nm})$ there is absorption as indicated by the transmission being close to 0 . For wavelengths above the bandgap the transmission goes to around 1. The oscillations in the transmission spectra arise from the interference due to the thickness of the sample and correspond to the interference expression $^{88}$ :

$$
m \lambda=2 n s
$$

In this equation $m$ is an integer value for the fringe maxima and half integer for fringe minima, $\lambda$ is the wavelength, $n$ is the refractive index, and $s$ is the thickness of the thin film. The thickness of the film can be extracted by plotting $1 / \lambda$ for each minima and maxima as a function of $m$ as shown in Figure 3-18. The slope of this line is equal to $1 / 2 \mathrm{~ns}$. Therefore if the refractive index

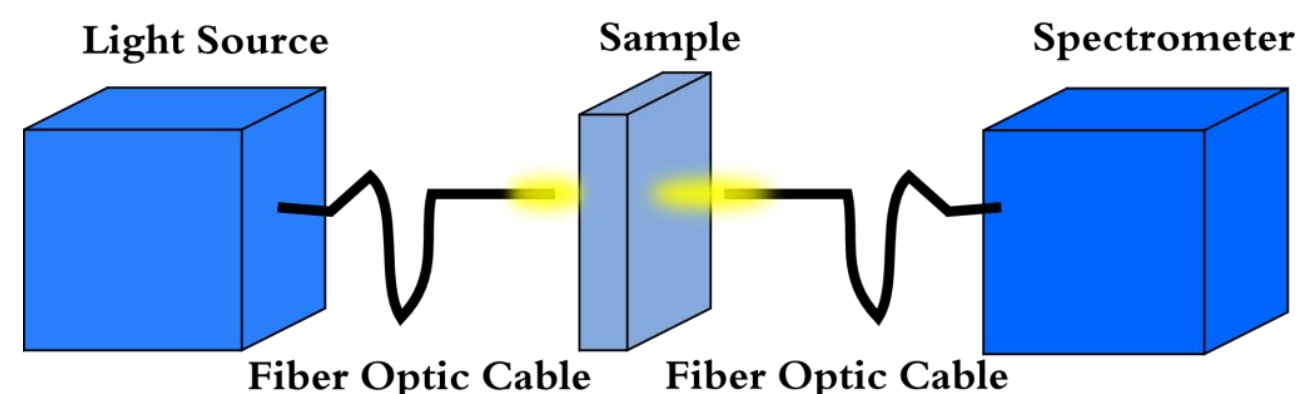

Figure 3-16 - Transmission setup using white light source, CCD spectrometer, and fiber optic cables. 


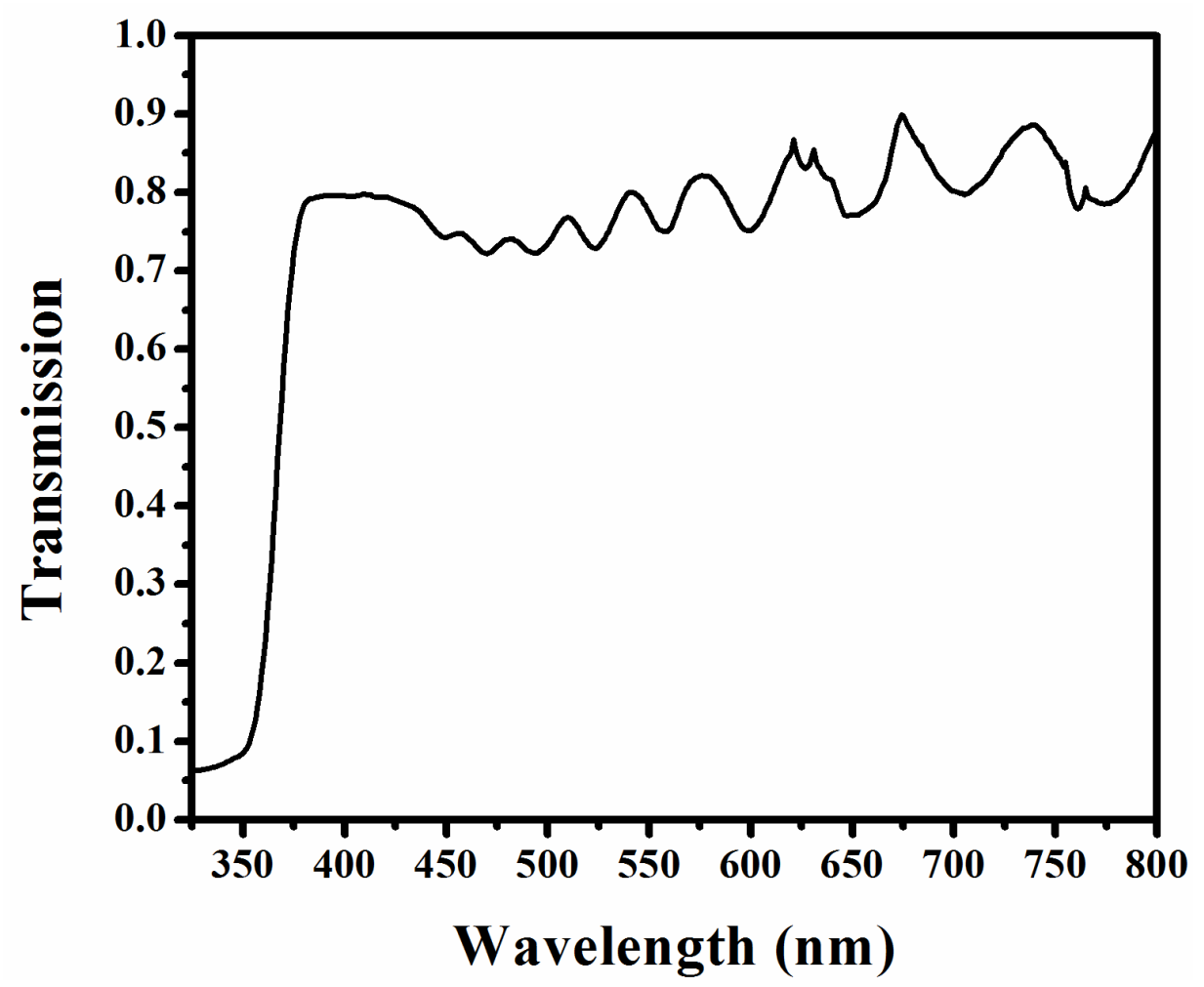

Figure 3-17 - Transmission of GaN film on sapphire.

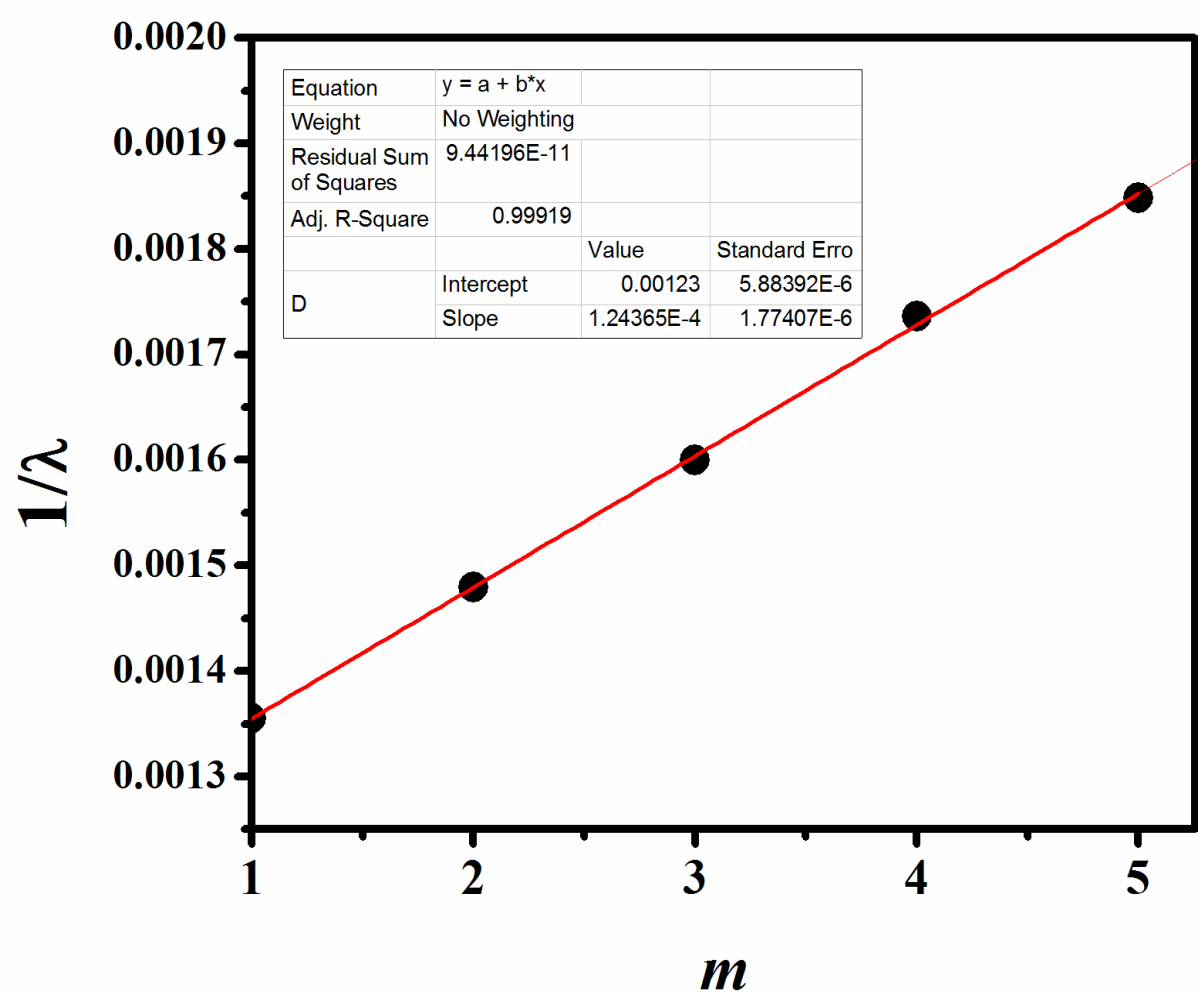

Figure 3-18 - Plot of interference fringes in transmission of GaN on sapphire sample. 
is know, the thickness can be extracted, or if the thickness is well known, the refractive index can be extracted. Assuming a refractive index of 2.44 for GaN, Figure 3-18 indicates the thickness of the film was around $1648 \mathrm{~nm}$.

Transmission can also provide useful information about the bandgap of the thin film. The intensity of light transmitted through the sample depends on the absorption coefficient and is given by ${ }^{89}$ :

$$
I_{t}=I_{o} e^{-\alpha s}
$$

In this expression, $I_{t}$ is the intensity of the transmitted light, $I_{o}$ is the incident light intensity, $\alpha$ is the absorption coefficient, and $s$ is the sample thickness. The absorption coefficient is related to the bandgap in the following equation ${ }^{89}$ :

$$
\alpha(E) \propto K \sqrt{E-E g}
$$

In this expression, $E$ is energy, $E_{g}$ is the bandgap, and $K$ is a proportionality constant. From this equation, $\alpha$ has a square root dependence on $E$ and therefore $\alpha^{2}$ has a linear dependence on $E$. The bandgap can be found by plotting $\alpha^{2}$ as extracted from Eq. 3-4 as a function of $E$. In other words, plot $\left(\frac{\left(\frac{I t}{I_{o}}\right)}{d}\right)^{2}$ as a function of energy. In this plot there is a linear region over which a linear fit can be performed to find the bandgap as shown in Figure 3-19. The value at which this line intersects the $\mathrm{x}$-axis is the bandgap. For a more consistent method, the line can be fit tangent to the point where the slope is a maximum.

\subsection{Reflectivity Measurements}

Reflectivity measurements were used in this work for the characterization of the DBR response. A y-shaped, FRP Series Fiber Reflectance Probe was used for this measurement as shown in Figure 3-20. One end of the fiber optic was connected to a BDS-100 tungsten/deuterium white light lamp, one end to the CCD spectrometer, and the third end was oriented normal to the sample surface. The fiber optic cable has a bundle of 7 optical fibers in a stainless steel ferrule at the sample end. Six fibers connect to the light source to deliver the light, while one fiber feeds back to the spectrometer. The reflectivity of the sample was taken and 


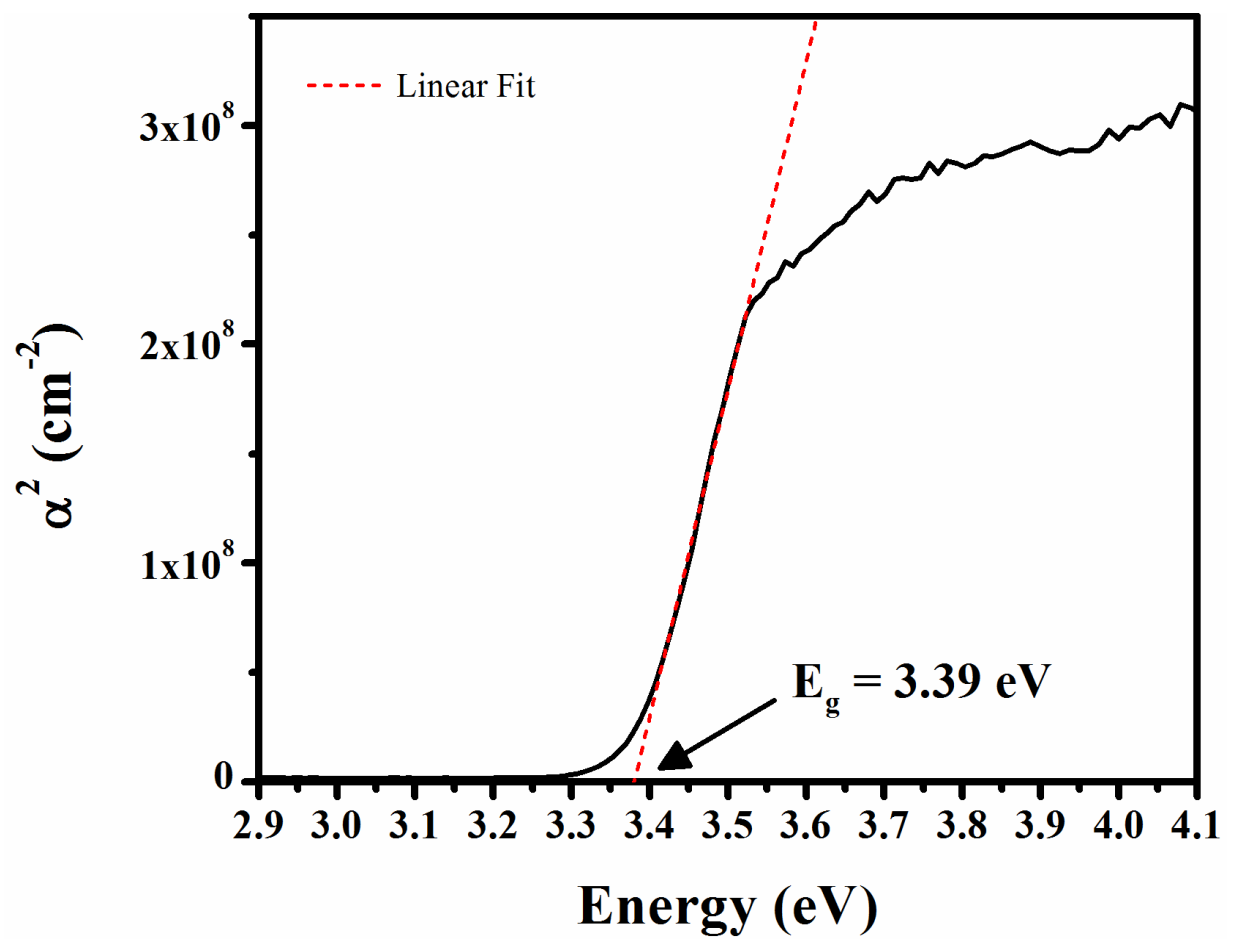

Figure 3-19-Extraction of bandgap from transmission measuremetns.

Light Source

Spectrometer
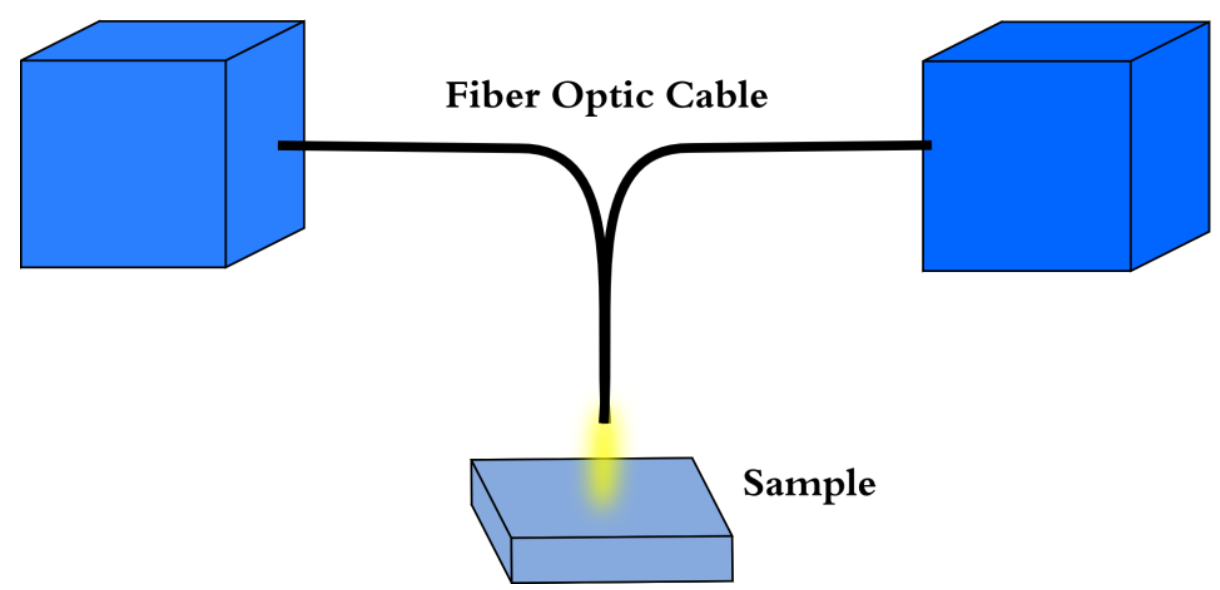

Figure 3-20 - Setup for measurement the reflectivity of a sample at normal incidence. 
divided by the reflectivity measurement from the aluminum coated pyrex mirror. The aluminum coated pyrex mirror is not a perfect reflector and therefore the measurements must be multiplied by the reflectivity of the mirror which is shown in Figure 3-21. An example of a DBR reflectivity spectrum is shown in Figure 3-22.

\subsection{Ellipsometry}

Ellipsometry is a characterization technique that measures the change in polarization of light that is reflected from a thin film in order to characterize the optical properties of thin films ${ }^{90}$. Light can be separated into a p-component in which the fields are parallel to the plane of incidence and an s-component in which the fields are perpendicular to the plane of incidence. Ellipsometry measures how these two components of light change upon reflection from a thin film surface. Two polarization states by the following ${ }^{90}$ :

$$
\rho=\frac{R_{p}}{R_{s}}=\tan (\psi) e^{i \Delta}
$$

This equation relates the ratio of reflections in the $p$ and $s$ directions to determine the amplitude of reflected wave, $\tan (\Psi)$, and the phase of the reflected wave, $\Delta$. From this information, various models can be develops and used to fit the data. Variable Angle Spectroscopic Ellipsometry (VASE) is used to collect reflection data from samples at multiple angles and multiple wavelengths in order to characterize the optical properties. A flow chart of an ellipsometry technique $^{91}$ is shown in Figure 3-23. The sample is first measured and then fit to an optical model. The model is adjusted until a fit between the experimental data and model data is achieved with a low mean square error. In this work, ellispsometry is used to extract the refractive index from III-Nitride based films. Measurements were taken with an M-2000U Variable Angle Spectroscopic Ellipsometer and modeled using WVASE32 software, both from J.A. Woollam Co. Inc. The Cauchy dispersion formula was used for modeling the layers. 


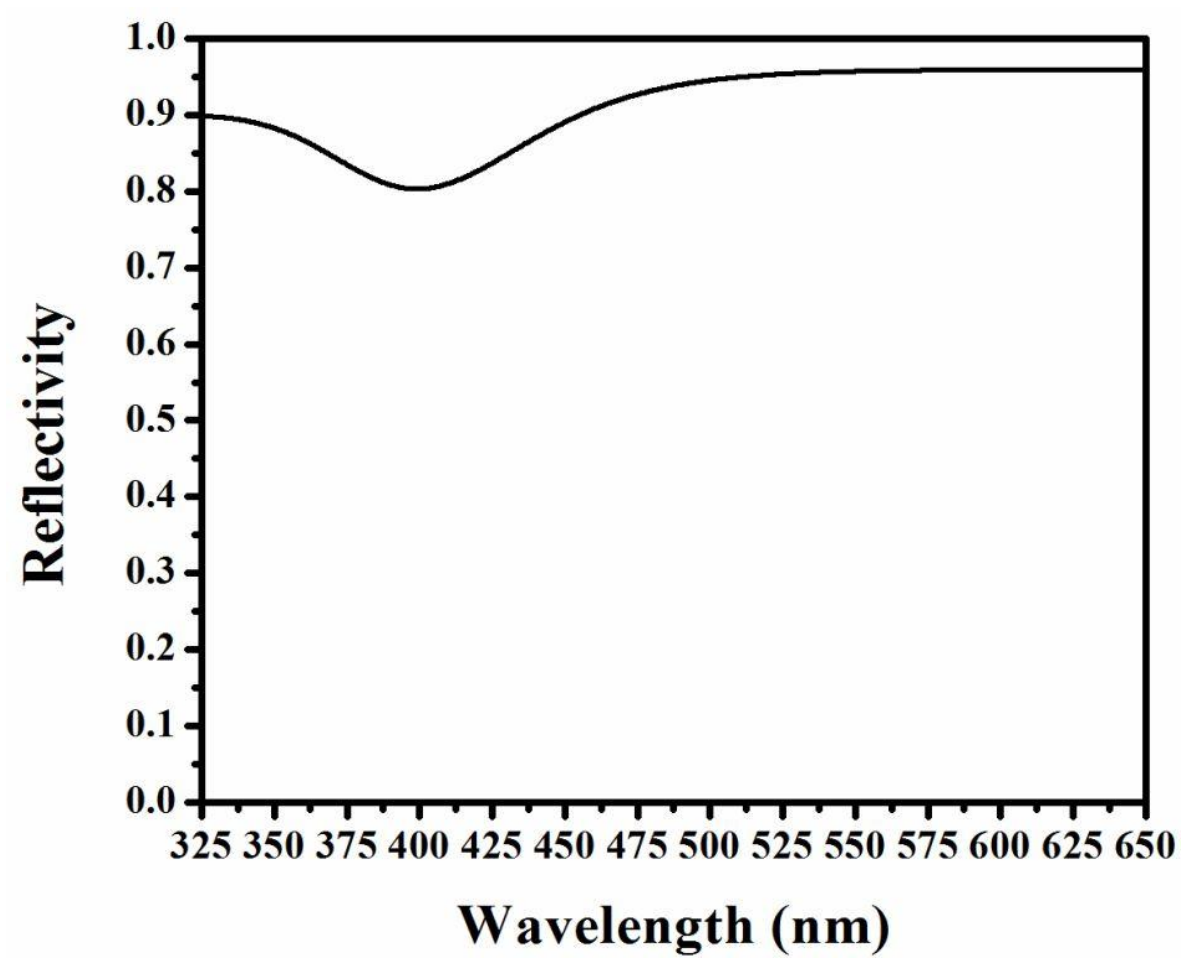

Figure 3-21 - Reflectivity of the aluminum coated pyrex mirror used in for reflectivity measurements in this work.

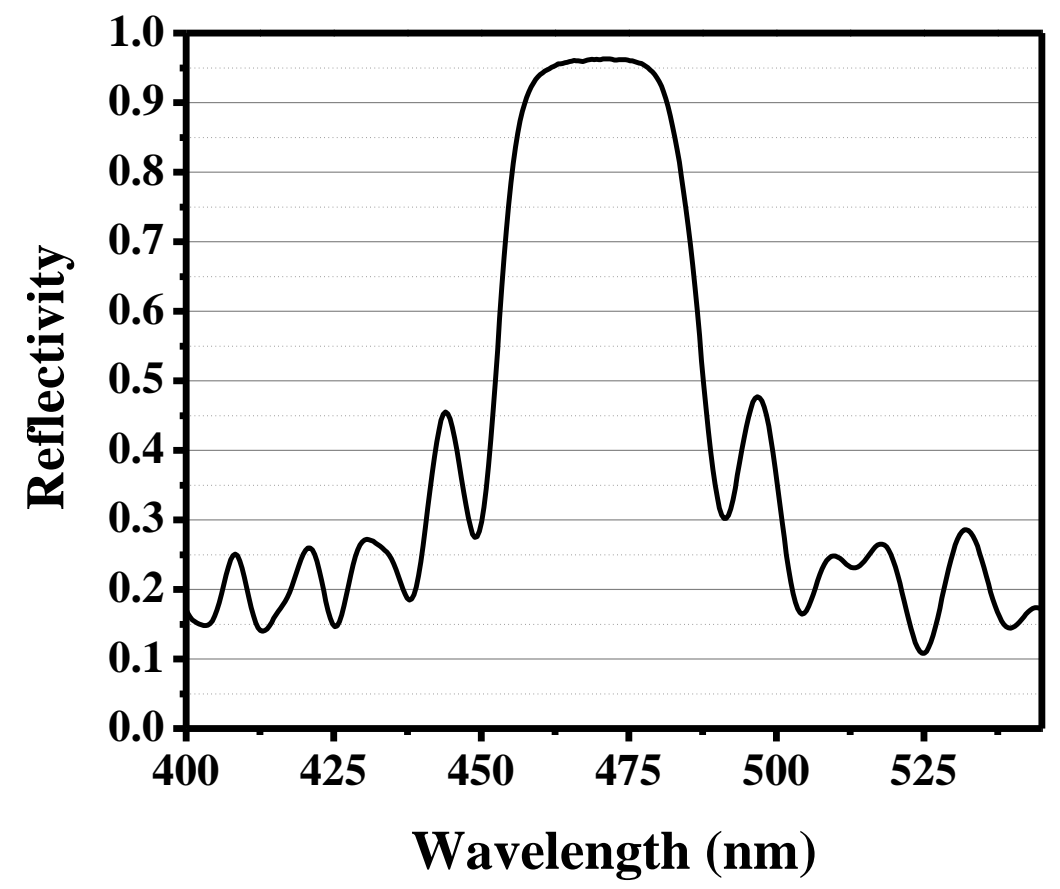

Figure 3-22 - Reflectivity of an III-Nitride based DBR. 


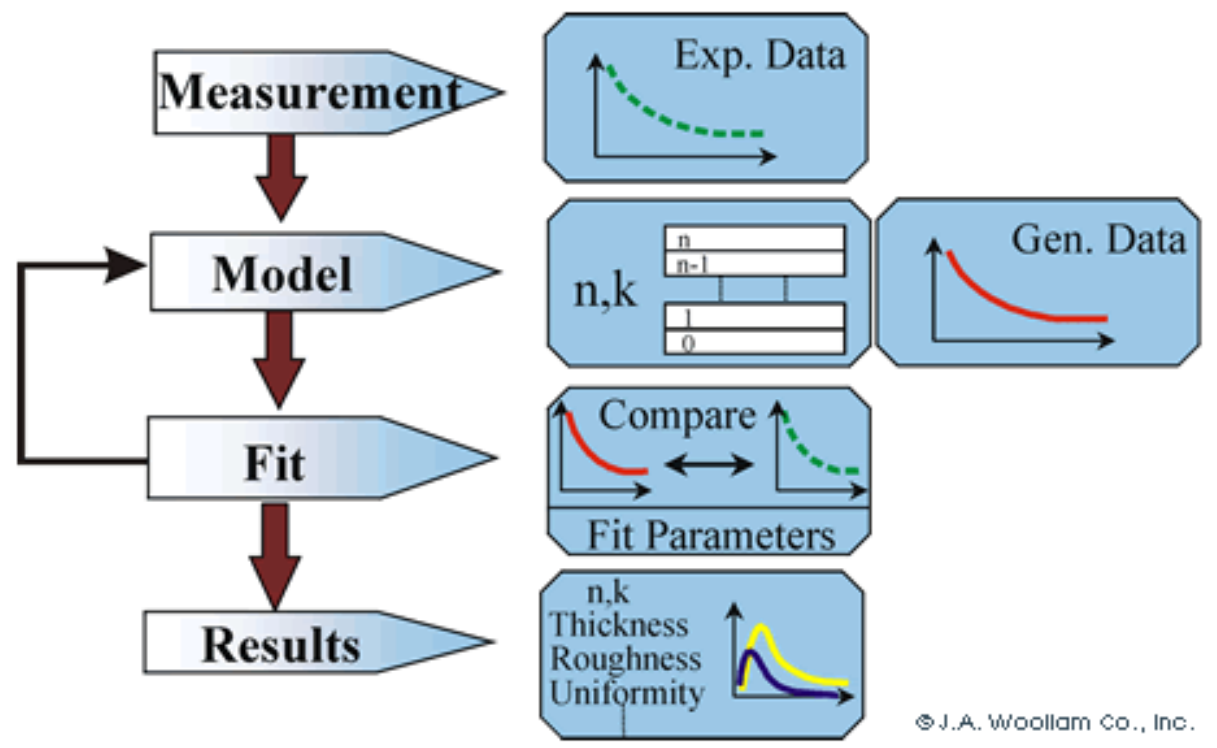

Figure 3-23 - Ellipsometry measurement techinque process flow. From Ref. 91

\subsection{Hall Measurements}

Hall Effect Measurements are used to verify the carrier concentration and corresponding mobility in $n$-type and $p$-type materials. Hall measurements are performed by applying a magnetic field perpendicular to the direction of current flow in a bar shaped semiconductor. The Lorentz forces on the carriers due to the applied magnetic field deflect the carriers to one side of the sample. The total force due to the applied electric field and also the magnetic field is given by $^{92}$ :

$$
F=q(E+v \times B)
$$

In which $q$ is the charge of the carrier, $E$ is the electric field, $v$ is the velocity of the carriers, and $B$ is the magnetic field. This force causes carriers to accumulate along one side of the semiconductor creating an electric field, $E_{y}$, which is perpendicular to both the current flow and also the magnetic field. This new electric field will balance the Lorentz force created by the magnetic field and the remainder of the carriers will experience no net lateral force as they drift through the material. This induced electric field, $E_{y}$, is known as the Hall Effect and is directly related to the measurable Hall Voltage and Hall coefficient. From these quantities, the carrier 
concentration and mobility can be extracted ${ }^{92}$. The measured Hall voltage, $V_{H}$, is given by the following ${ }^{92}$.

$$
V_{H}=E_{y} w
$$

Where $w$ is the width of the semiconductor sample. The Hall coefficient can then be calculated from by:

$$
R_{H}=\frac{V_{H} t}{I B}
$$

In which $t$ is the thickness of the sample, $I$ is the current, and $B$ is the magnetic field. The carrier concentration of holes $(p)$ or electrons $(n)$ can then be calculated by the following:

$$
p(n)=+(-) \frac{1}{q R_{H}}
$$

Using a resistance measurement $R$, the resistivity of the sample can be found by knowing the sample cross section $(A)$ and also the length $(l)$ :

$$
\rho=\frac{R A}{l}
$$

From the resistivity, the mobility can then be calculated as:

$$
\mu=\frac{\left|R_{H}\right|}{\rho}
$$

The calculations above correspond to a bar type configuration which is very sensitive to sample shape and contact placement. The Van der Pauw technique is more commonly used in order to accommodate irregular shaped samples. In this technique ${ }^{93}$, four contacts are placed on the periphery of the sample as shown in Figure 3-24. The resistance can be calculated as $\mathrm{R}_{12,34}=$ $\mathrm{V}_{34} / \mathrm{I}_{12}$. The notation indicates that the voltage is measured between terminals 3 and 4 while current is driven from terminal 1 to 2 . Measuring the different configurations, two resistances can be calculated by averaging four measurements ${ }^{93}$ :

$$
\begin{aligned}
& R_{\mathrm{A}}=\left(R_{21,34}+R_{12,43}+R_{43,12}+R_{34,21}\right) / 4 \\
& R_{\mathrm{B}}=\left(R_{32,41}+R_{23,14}+R_{14,23}+R_{41,32}\right) / 4
\end{aligned}
$$




$$
V_{\mathrm{H}}=V_{24 \mathrm{P}}
$$

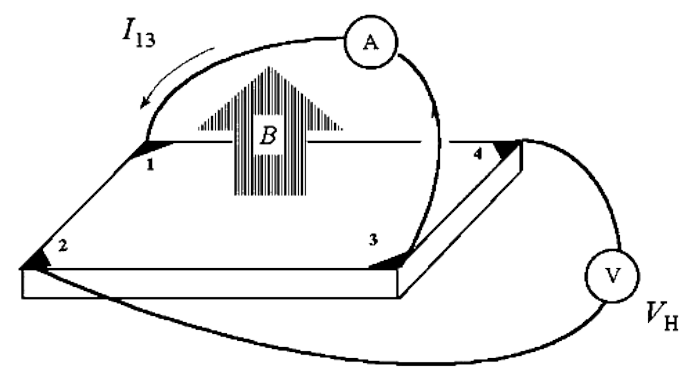

Figure 3-24 - Schematic of Van Der Pauw contact geometry. Image from Ref. 93.

The sheet resistance can be calculated by solving the following expression for $\mathrm{Rs}^{93}$.

$$
\exp \left(-\pi R_{\mathrm{A}} / R_{\mathrm{S}}\right)+\exp \left(-\pi R_{\mathrm{B}} / R_{\mathrm{S}}\right)=1
$$

As before, the Hall voltage must be measured in the presence of a magnetic field. The procedure is to apply a magnetic field and then measure the voltage from two terminals while driving current through the other two. Specifically voltages $V_{24 \mathrm{P}}, V_{42 \mathrm{P}}, V_{13 \mathrm{P}}$, and $V_{31 \mathrm{P}}$, with $I_{13}, I_{31}, I_{42}$, and $I_{24}$, respectively must be measured. The subscript $\mathrm{P}$ indicates a positive magnetic field. In this measurement, the voltage between terminals 2 and 4 is measured while current is driven between terminals 1 and 3 . Likewise the voltages $V_{24 \mathrm{~N}}, V_{42 \mathrm{~N}}, V_{13 \mathrm{~N}}$, and $V_{31 \mathrm{~N}}$ with $I_{13}, I_{31}, I_{42}$, and $I_{24}$, respectively need to be measured. The subscript $\mathrm{N}$ indicates the opposite magnetic field. The differences between the voltages can be calculated as follows ${ }^{93}$ :

$$
\begin{aligned}
& V_{\mathrm{C}}=V_{24 \mathrm{P}}-V_{24 \mathrm{~N}} \\
& V_{\mathrm{D}}=V_{42 \mathrm{P}}-V_{42 \mathrm{~N}} \\
& V_{\mathrm{E}}=V_{13 \mathrm{P}}-V_{13 \mathrm{~N}} \\
& V_{\mathrm{F}}=V_{31 \mathrm{P}}-V_{31 \mathrm{~N}}
\end{aligned}
$$

If the sum of these four voltages is positive, then the semiconductor is $p$-type. Accordingly, if the sum of these voltages is negative, the semiconductor is $n$-type. The overall hall voltages is then $V_{H}=8 /\left(V_{\mathrm{C}}+V_{\mathrm{D}}+V_{\mathrm{E}}+V_{\mathrm{F}}\right)$. The sheet carrier density for holes or electrons is calculated by the following ${ }^{93}$ :

$$
p_{\mathrm{s}}=I B /\left[q V_{H}\right]
$$




$$
n_{\mathrm{s}}=\left|I B /\left[q V_{H}\right]\right|
$$

The bulk carrier concentration is calculated by dividing by the thickness of the sample.

$$
\begin{aligned}
& p=p_{\mathrm{s}} / d \\
& n=n_{\mathrm{s}} / d
\end{aligned}
$$

The mobility can then be calculated from the following equation using the sheet resistance and sheet carrier concentration:

$$
\mu=1 / q n_{\mathrm{S}} R_{\mathrm{S}}
$$

To verify the properties of the films grown at WVU, the Ecopia Hall Effect System was used. Samples were cut into squares and indium contacts were soldered to each corner. Gold wire was used to connect the sample to the mount for testing. Periodically, samples were measured in order to verify the operation of the MOCVD and also to optimize the layer properties for devices. Figure 3-26 shows the relationship between the bulk carrier concentration and the mobility for $n$-type GaN films. These films were doped with silicon which is achieved by introducing dilute silane $\left(\mathrm{SiH}_{4}\right)$ into the growth. Samples grown using both an AlN and GaN buffer layer are shown in this plot and follow the bell shaped trend explained in literature ${ }^{94}$. At carrier concentrations less than $\sim 5 \times 10^{17} \mathrm{~cm}^{-3}$, the mobility is limited by scattering with the dislocations whose density is represented by the dotted lines. Above this concentration, the mobility is influenced by ionized impurity scattering and decreases with increasing carrier concentration. It has been observed at WVU that when the $n$-type GaN layer is grown on a insulating $\mathrm{GaN}$ epilayer layer that is at least $3 \mu \mathrm{m}$ thick, the mobility increases from approximately $70 \mathrm{~cm}^{2} / \mathrm{Vs}$ to $200 \mathrm{~cm}^{2} / \mathrm{Vs}$. This corresponds to an approximate decrease in the dislocation density from $2 \times 10^{10} \mathrm{~cm}^{-2}$ to $8 \times 10^{9} \mathrm{~cm}^{-2}$. Therefore, for active devices a $\sim 3 \mu \mathrm{m} \mathrm{GaN}$ buffer layer was employed. The ratio of the silane supplied to the group III source supplied can be used to control the carrier concentration in the film. Figure 3-26 shows the linear relationship between the flows and the carrier concentration. Excess silicon doping will cause GaN to crack due to an increase in the $a$-lattice parameter with increasing silicon incorporation ${ }^{95}$. Bulk carrier concentrations around $1.5 \times 10^{19} \mathrm{~cm}^{-3}$ have been shown to cause cracking in films grown at WVU. 


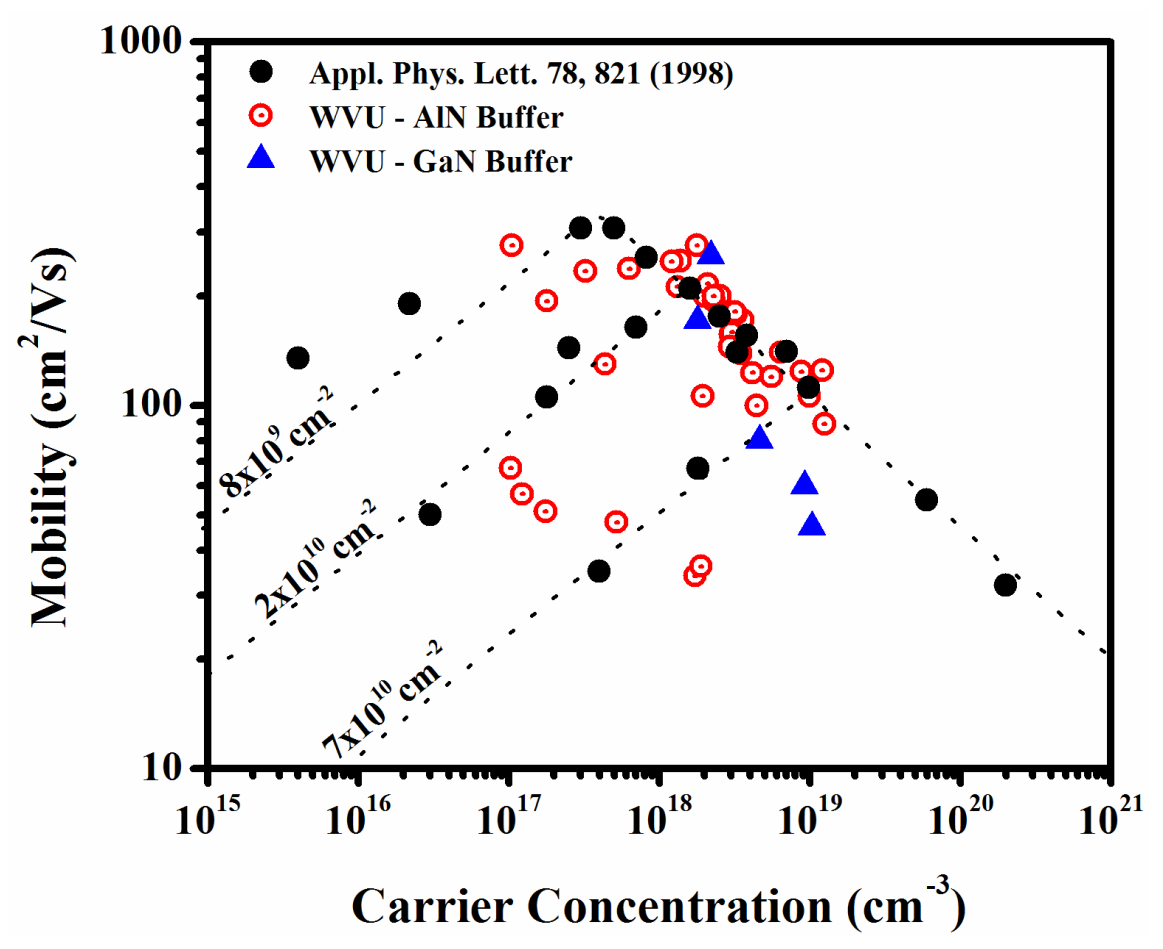

Figure 3-25 - Relationship between carrier mobility and bulk carrier concetration for $n$-type GaN.

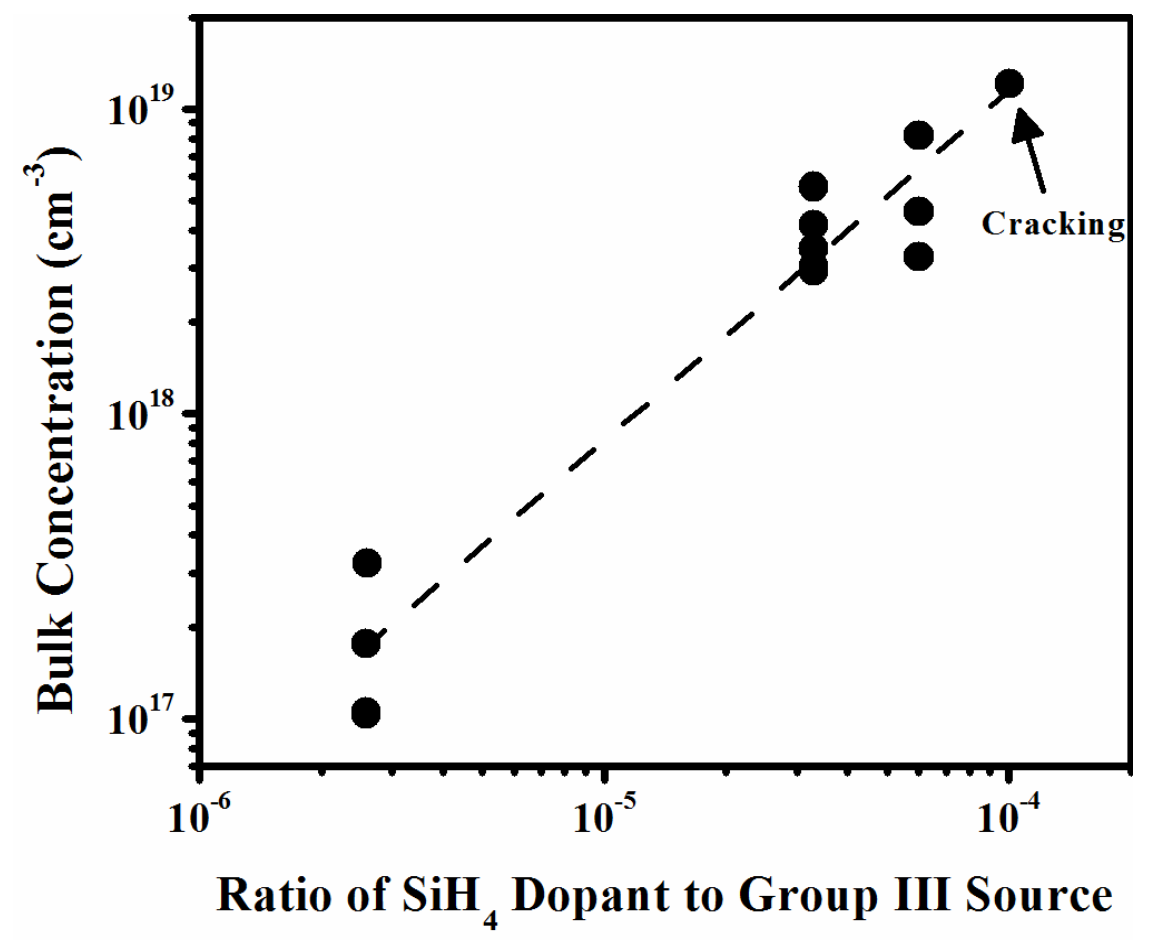

Figure 3-26-Bulk carrier concetration of n-type GaN as a function of the ratio of silane dopant to group III source supplied. 


\section{H A P T E R 4 - D I G I T A L A L L O Y G R O W T H}

\subsection{Introduction}

Digital alloys consist of short period superlattices in which the periodicity is a few monolayers. In this work, $\mathrm{Al}_{x} \mathrm{Ga}_{1-x} \mathrm{~N}$ alloys were grown using a digital technique. High quality $\mathrm{Al}_{x} \mathrm{Ga}_{1-x} \mathrm{~N}$ alloys, as already mentioned, are plagued by several growth challenges. Anytime multiple species are introduced into growth, the kinetics become considerably more complicated. In this work, a digital growth technique is targeted in order to alleviate many of the growth related challenges. Furthermore, the use of modulation doping of the superlattices ${ }^{96,97}$ can eventually lead to a conductive superlattice layer for integration in the DBR structure, although it is beyond the scope of this work. In this work, digital alloys have been fabricated by depositing thin layers of GaN followed by thin layers of AlN as shown in Figure 4-1a. By accurately controlling the number of monolayers in the superlattice, the AlN mole fraction in the $\mathrm{Al}_{x} \mathrm{Ga}_{1-x} \mathrm{~N}$ alloy can be precisely tuned.

The optimized growth conditions for $\mathrm{AlN}$ and GaN layers differ significantly. In particular, AlN is typically grown at considerably higher temperatures and lower reactor pressures when compared to GaN. As previously mentioned, this is to increase the mobility of the Al species which has a larger sticking coefficient than Ga and also to limit the parasitic reactions that occur between TMAl and $\mathrm{NH}_{3}{ }^{42}$. Therefore, it was the intent of this work to determine a single set of growth conditions that will yield high quality AlN and GaN very thin films. This will allow for fast switching between the layers in the superlattice. The growth temperature targeted in this work was $1000{ }^{\circ} \mathrm{C}$ because this results in reasonable quality AlN and also GaN based on previous results of individual thin film growth. However, other temperatures were also investigated. One of the most important parameters under investigation was the reactor pressure as it largely influences the AlN growth. Therefore, growth conditions have been identified that yield both high quality $\mathrm{AlN}$ and $\mathrm{GaN}$ film growth so that $\mathrm{Al}_{\mathrm{x}} \mathrm{Ga}_{1-\mathrm{x}} \mathrm{N}$ alloys with AlN mole fractions ranging from 0 to 1 have been grown by fixing the GaN thickness and changing the AlN thickness. 


\subsection{Digital Alloy Growth}

Digital alloys were grown in the Aixtron 200/4 RF-S MOVPE horizontal reactor. The $\mathrm{Ga}, \mathrm{Al}$, and $\mathrm{N}$ precursors used for growth were trimethylgallium (TMGa), trimethylaluminum (TMAl), and $\mathrm{NH}_{3}$, respectively. Single sided polished sapphire substrates with a 0.3 degree miscut toward the $m$-plane were used. Prior to growth the sapphire was cleaned in situ at $900{ }^{\circ} \mathrm{C}$ under hydrogen. This cleaning was followed by a $40 \mathrm{~nm}$ thick AlN nucleation layer deposited directly on the sapphire. Next, either a $50 \mathrm{~nm}$ thick GaN or $200 \mathrm{~nm}$ thick AlN buffer layer was grown. The AlN buffer layer was chosen to be thicker than the GaN buffer layer in order to obtain reasonable intensity in subsequent XRD characterization which will be discussed later. After the buffer layer growth, the AlN/GaN superlattice was grown at $1000{ }^{\circ} \mathrm{C} . \quad$. $40 \mu \mathrm{mol} / \mathrm{min}$ of TMAl was supplied for the AlN growth and $80 \mu \mathrm{mol} / \mathrm{min}$ of TMGa was supplied for the GaN growth. $1.2 \mathrm{slpm}$ of $\mathrm{NH}_{3}$ was supplied to the reactor during growth. In this work, the GaN growth time was fixed at 1 second while the AlN growth time was varied from 2 seconds to 188 seconds ${ }^{98,99}$. All of the samples, except those with an AlN mole fraction of 0.8 and above consist of 300 periods. For practical reasons, samples with an AlN mole fraction greater than 0.8 contain between 100-150 periods. In order to ensure minimal precursor mixing and alloy deposition, a three second $\mathrm{NH}_{3}$ purge was used in between the GaN and AlN growth. Figure 4-1 illustrates the structure under investigation and also the growth scheme used in this work. Several growth pressures were investigated as shown in Figure 4-2. At 200 mbar, which is the optimized GaN growth pressure, there was no AlN deposition using this pulse flow techinque as indicated by the absence of the $\mathrm{Al}_{x} \mathrm{Ga}_{1-x} \mathrm{~N}(0002)$ in the $2 \theta-\omega$ scan. At 50 mbar, which is the AlN growth pressure, the digital alloys were characterized by a high density of cracks and also the (0002) peak was split indicating non-uniform composition. Both pressures of 65 mbar and 100 mbar resulted in very minimal cracking and sharp $\mathrm{Al}_{x} \mathrm{Ga}_{1-x} \mathrm{~N}$ (0002) peaks. For this work, 65 mbar was chosen due to the slow growth rate of $\mathrm{AlN}$ at 100 mbar. This set of growth conditions is a compromise between the GaN and $\mathrm{AlN}$ growth conditions for single films. 

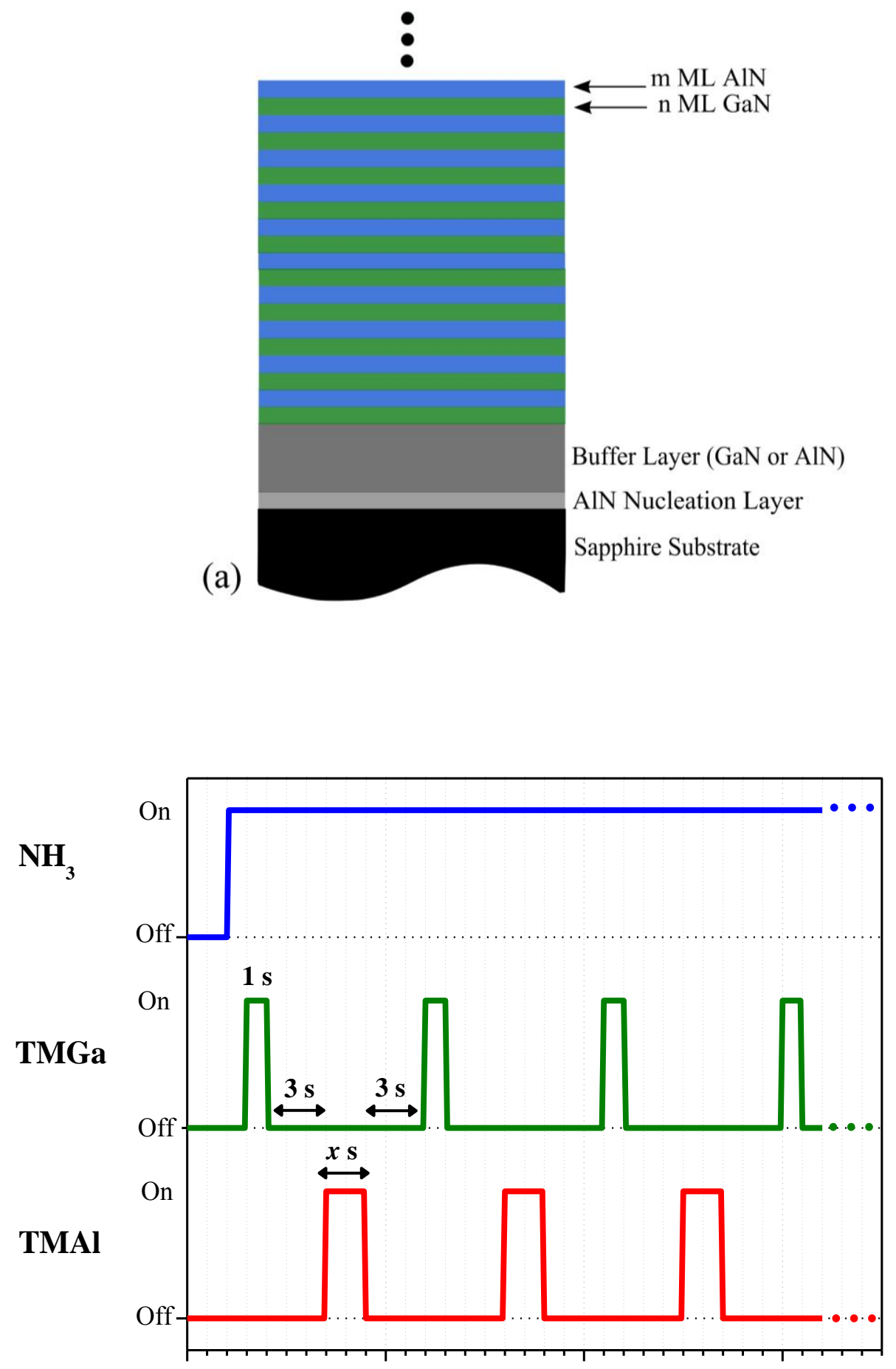

Time (s)

Figure 4-1 - (a) Schematic of $A l_{\mathrm{x}} G a_{1-\mathrm{x}} N$ digital alloy on sapphire substrate and (b) pulse flow scheme for growth. 

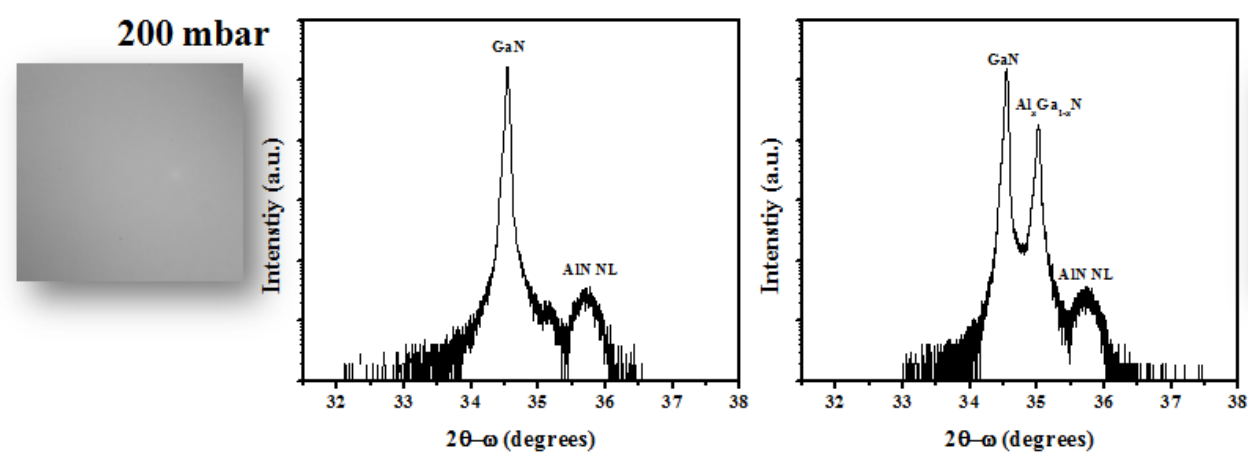

100 mbar
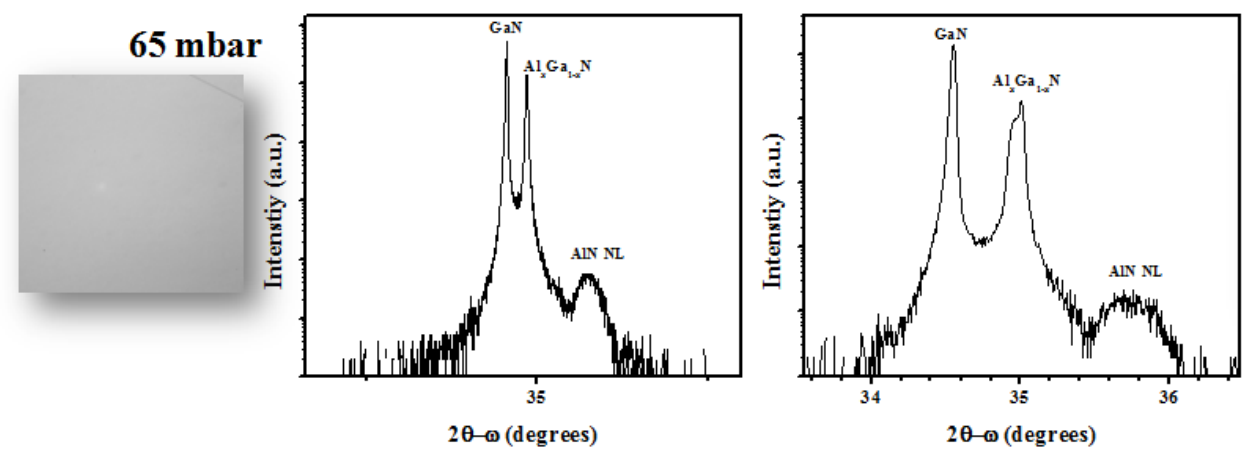

\section{0 mbar}

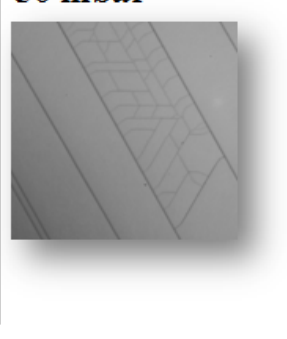

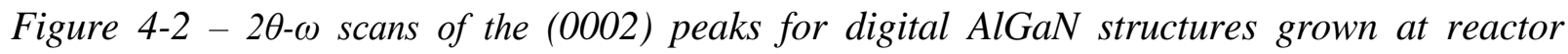
pressures of 200, 100, 65, and 50 mbar.

\subsection{Digital Alloy Characterization}

The III-Nitrides have a wurtzite structure and are therefore characterized by an $a$ - and $c$ lattice parameter. In this work the $c$-lattice parameter was accurately determined using the symmetric (0002), (0004), and (0006) reflections. The apparent lattice parameters derived from these individual reflections was plotted as a function of $\cos ^{2}(\theta) / \sin (\theta)$ as shown in Figure 4-3 $3^{100}$. Since these points are in a straight line, the largest source of error in the measurement is the eccentricity of the sample. The y-intercept of a linear fit of these points yielded the accurate $c$ lattice parameter. Off-axis peaks must be used to determine the $a$-lattice parameter and in this work, the (1014) reflection was used. In the hexagonal crystal structure, the relationship between lattice spacing $d$ for a given plane $(h k l)$, and the $c$ - and $a$-lattice parameters is as follows ${ }^{87}$ :

$$
\frac{1}{d^{2} \mathrm{hkl}}=\frac{4}{3} \frac{h^{2}+k^{2}+h k}{a^{2}}+\frac{l^{2}}{c^{2}}
$$


Using the $c$-lattice parameter as previously determined, it was possible to extract the $a$-lattice parameter from the (1014) lattice spacing.

$2 \theta-\omega$ scans of the (0002) reflections of the superlattices contained satellite peaks arising from the periodicity of the structure. The period, $T$, of the was thereby determined from the spacing between the primary and the $+/-1^{\text {st }}$ order satellite peaks using the following $\operatorname{expression}^{87}$ :

$$
T=\frac{\lambda}{2 \sin \left(\theta_{1}\right)}-\frac{\lambda}{2 \sin \left(\theta_{2}\right)}
$$

In this expression, $\lambda$ is the wavelength of the $\mathrm{x}$-rays and $\theta$ is the position of the satellite and primary peaks. Figure 4-4 shows a representative $2 \theta-\omega$ scan of the (0002) peak of an $\mathrm{Al}_{x} \mathrm{Ga}_{1-x} \mathrm{~N}$ digital alloy grown on a GaN buffer layer. In this figure the primary satellite peak, $+/ 1^{\text {st }}$ order

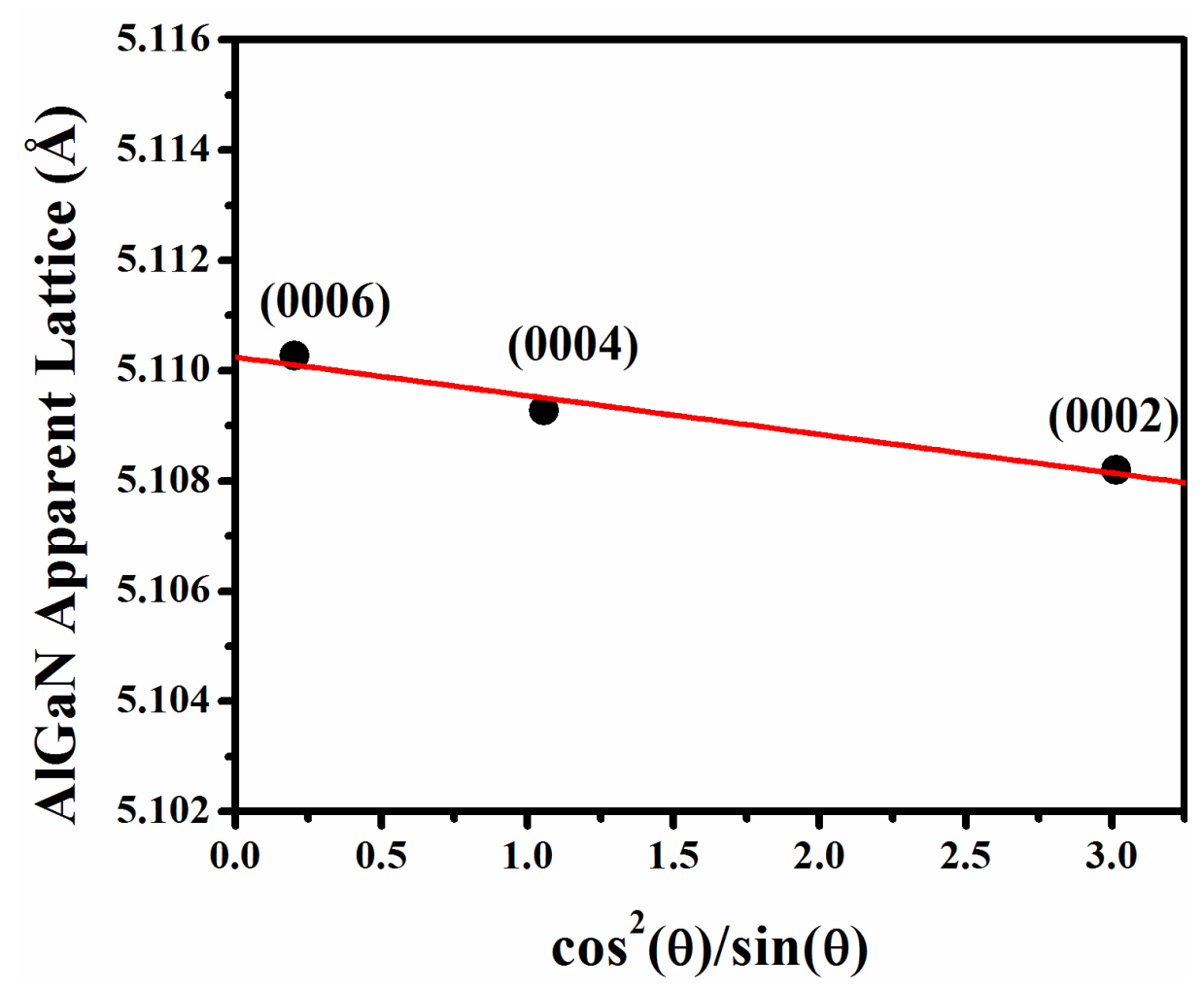

Figure 4-3 - Apparent lattice parameter of $\mathrm{Al}_{\mathrm{x}} \mathrm{Ga} a_{1-\mathrm{x}} \mathrm{N}$ digital alloys calculated from the (0002), (0004), and (0006) reflections. 


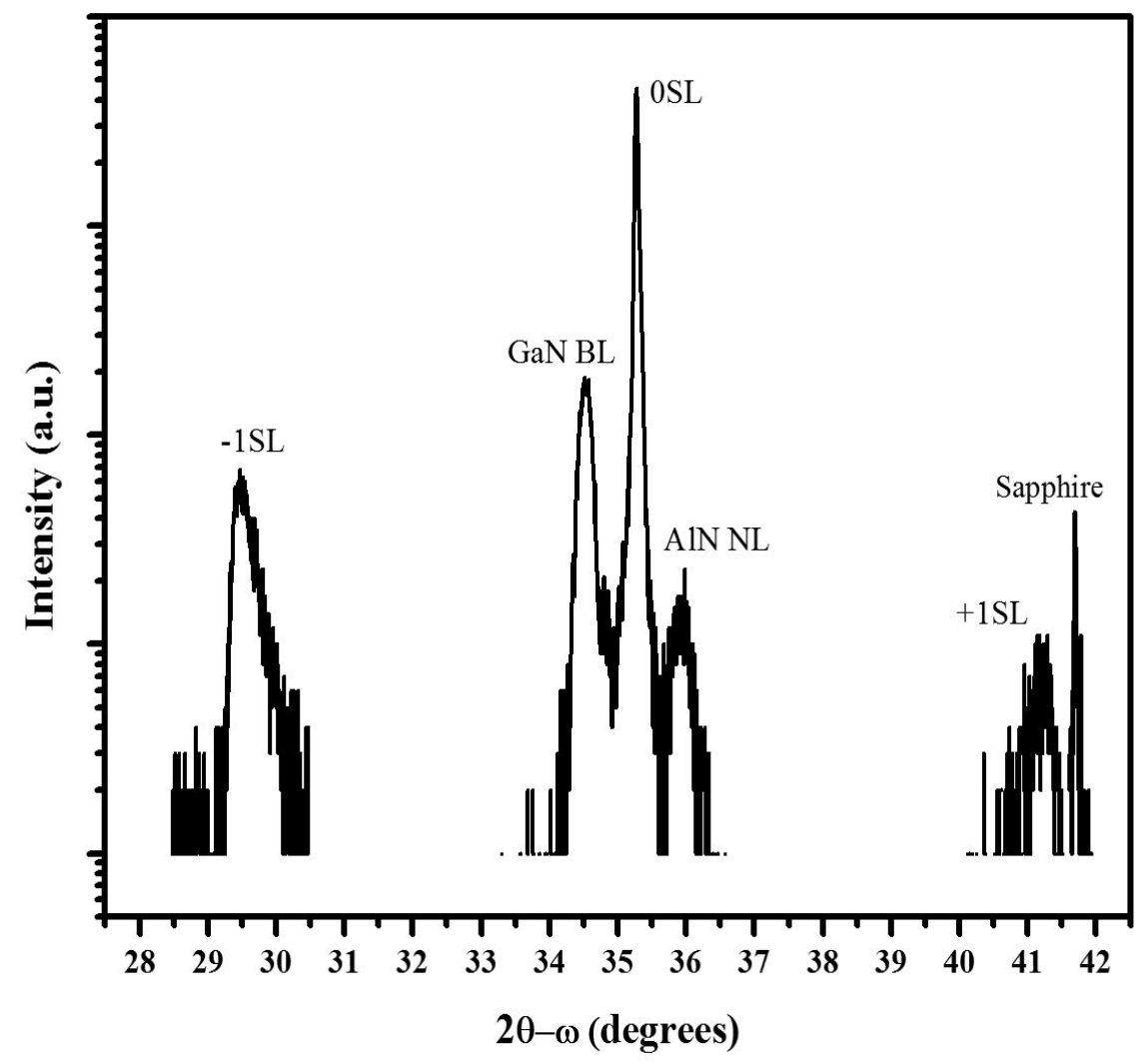

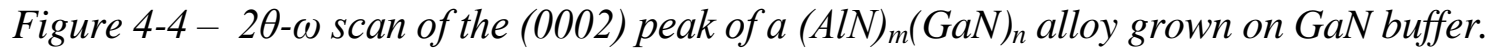

satellite peaks, GaN buffer peak, and AIN nucleation layer peaks are visible. The presence of the first order satellite peaks indicates the presence of the superlattice structure. Most likely due to the large periodicity, weak intensity, or interface quality, the higher order satellite peaks were not observed. This is in contrast to some other digital techniques in which apparent intermixing was observed $^{41}$.

Figure 4-5 contains the reciprocal space mappings for two representative samples. Figure 4-5a corresponds to an $\mathrm{AlN} / \mathrm{GaN}$ superlattice grown on the $\mathrm{GaN}$ buffer layer in which the superlattice is coherent with the buffer layer. This is indicated by the vertical alignment of the two peaks. Figure $4-5 b$ contains the mapping of a superlattice with thicker AlN layers also grown on the GaN buffer layer. As evident from the vertical misalignment of the two peaks, this superlattice is not coherent with the underlying buffer layer. The buffer layers used in this work were not fully relaxed because of the large lattice and thermal mismatch between the layers and the sapphire substrate ${ }^{101}$ and their thickness. The measured in-plane lattice parameter for the 

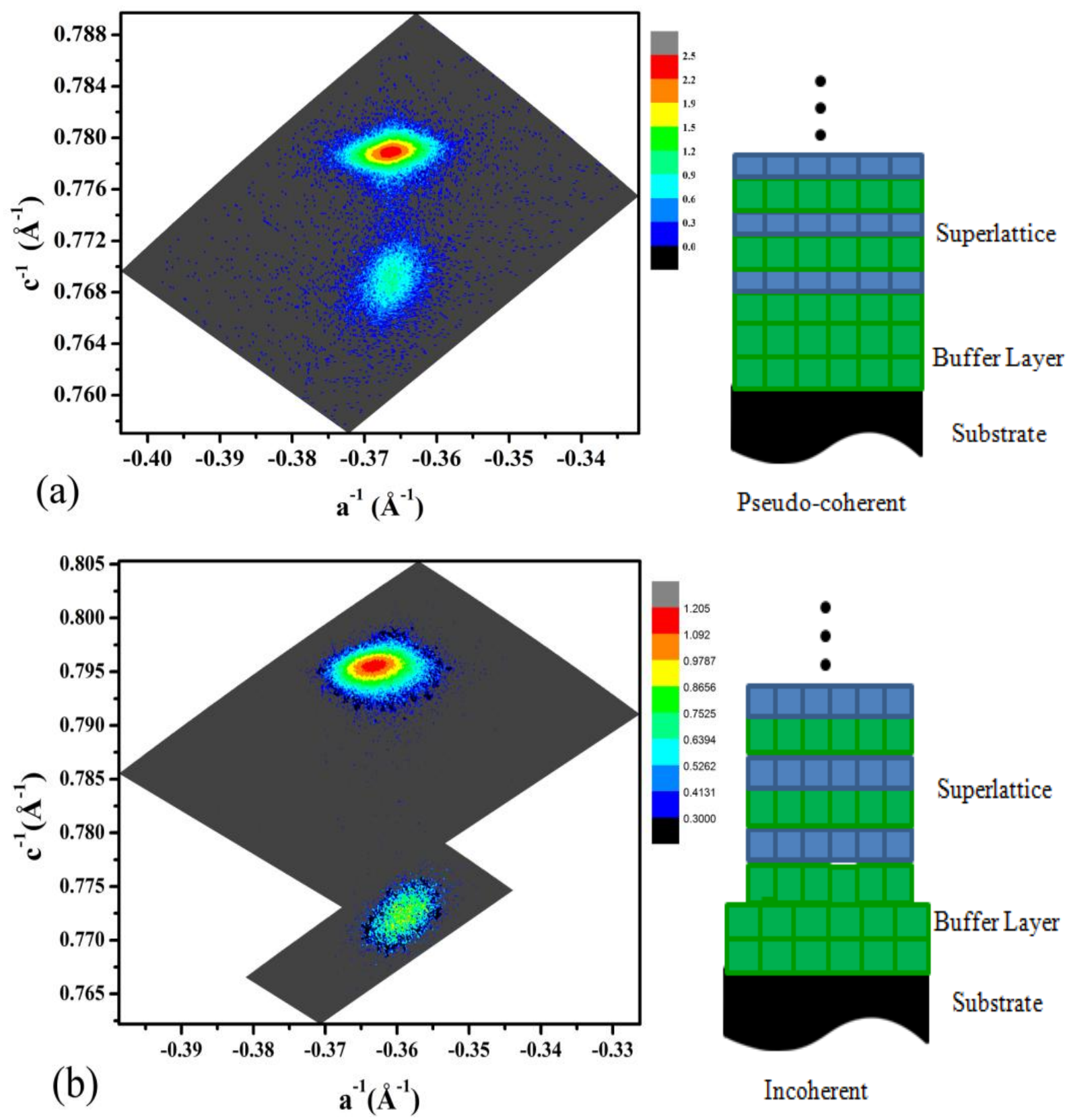

Pseudo-coherent
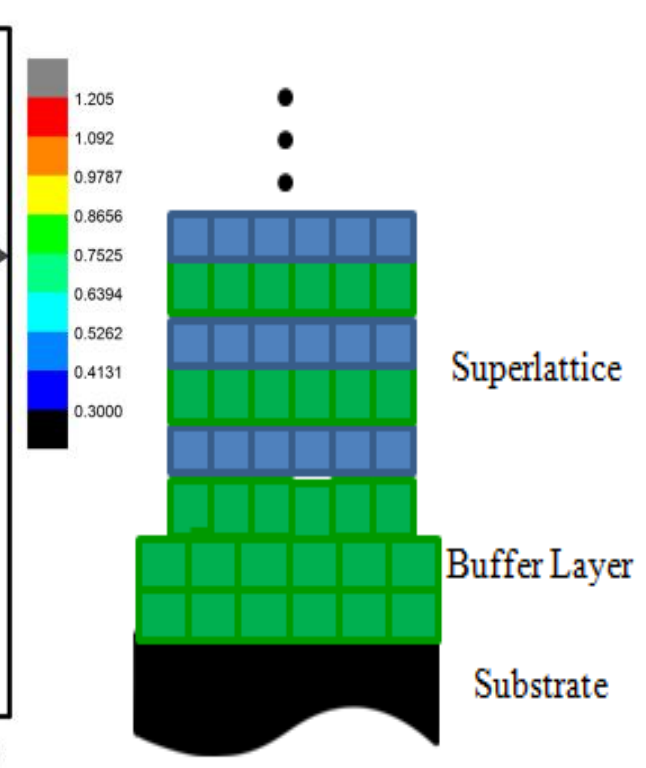

Figure 4-5 - Reciprocal space map of (1014) peak fortwo different $(A l N)_{m}(G a N)_{n}$ alloys grown on GaN buffer. (a) Shows a psuedocoherent film and (b) shows a relax film. 
GaN buffer layer was slightly less than nominal indicating compressive strain. On the other hand, the in-lattice parameter of the AIN buffer layer was slightly larger than nominal. This may be due to the growth conditions employed since it is well known that the growth kinetics largely influence relaxation in III-Nitride films ${ }^{123}$.

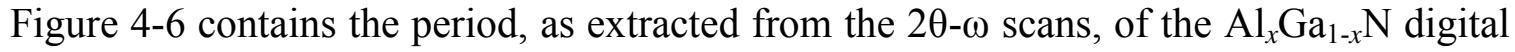
alloy as a function of the AlN growth time for samples grown on a GaN buffer layer and on an AlN buffer layer. As already mentioned, for each sample, the GaN growth time was 1 second. To obtain alloys of different concentrations, the AlN growth time was increased from 2 second up to 188 seconds. It can be seen from Figure 4-6 that this time range corresponds to a superlattice period varying linearly from approximately $10 \AA$ to $95 \AA$ and is independent of the buffer layer. The error in the measurement of the period ranges from approximately $2 \times 10^{-3} \AA$ to $2 \times 10^{-2} \AA$ and is dominated by the sometimes irregular shape of the satellite peaks. The error bars are small compared with the measurement and therefore are not visible in the figure. A linear fit of the data yields an AlN growth rate of approximately $0.45 \AA / \mathrm{s}$. The y-intercept from the linear fit yields a GaN thickness per period of approximately $9.4 \AA$. The thicknesses of the $\mathrm{GaN}$ layers do not correspond to the expected integer value of a monolayer. This type of discrepancy has been reported before in $\mathrm{Al}_{x} \mathrm{Ga}_{1-x} \mathrm{~N} / \mathrm{AlN}$ short period superlattices by Chandolu et $a^{102}$. In that work, potential explanations for the discrepancies include stacking faults, inversion domain boundaries, strain in the superlattice, composition fluctuations, intermixing, and/or interface roughness. In this work, the influence of stacking faults and inversion domain boundaries are neglected because stacking faults do not influence the $2 \theta-\omega$ scans and inversion domain boundaries are primary found in films doped with magnesium. The superlattices under investigation contain binary alloys $(\mathrm{AlN} / \mathrm{GaN})$ and therefore composition fluctuation is also not a concern. Chandolu et al. concluded for $\mathrm{Al}_{x} \mathrm{Ga}_{1-x} \mathrm{~N} / \mathrm{AlN}$ short period superlattices grown via MBE, interface roughness and screw dislocations are largely responsible for the observed discrepancy as they were able to rule out strain. However, in this work, we cannot rule out biaxial strain. Therefore, it is our best estimate that the discrepancy in measured thickness is due to a combination of strain, interface roughness, and dislocations. Figure 4-7 contains the effective AlN mole fraction for the $\mathrm{Al}_{x} \mathrm{Ga}_{1-x} \mathrm{~N}$ digital alloys. This is a calculated concentration 


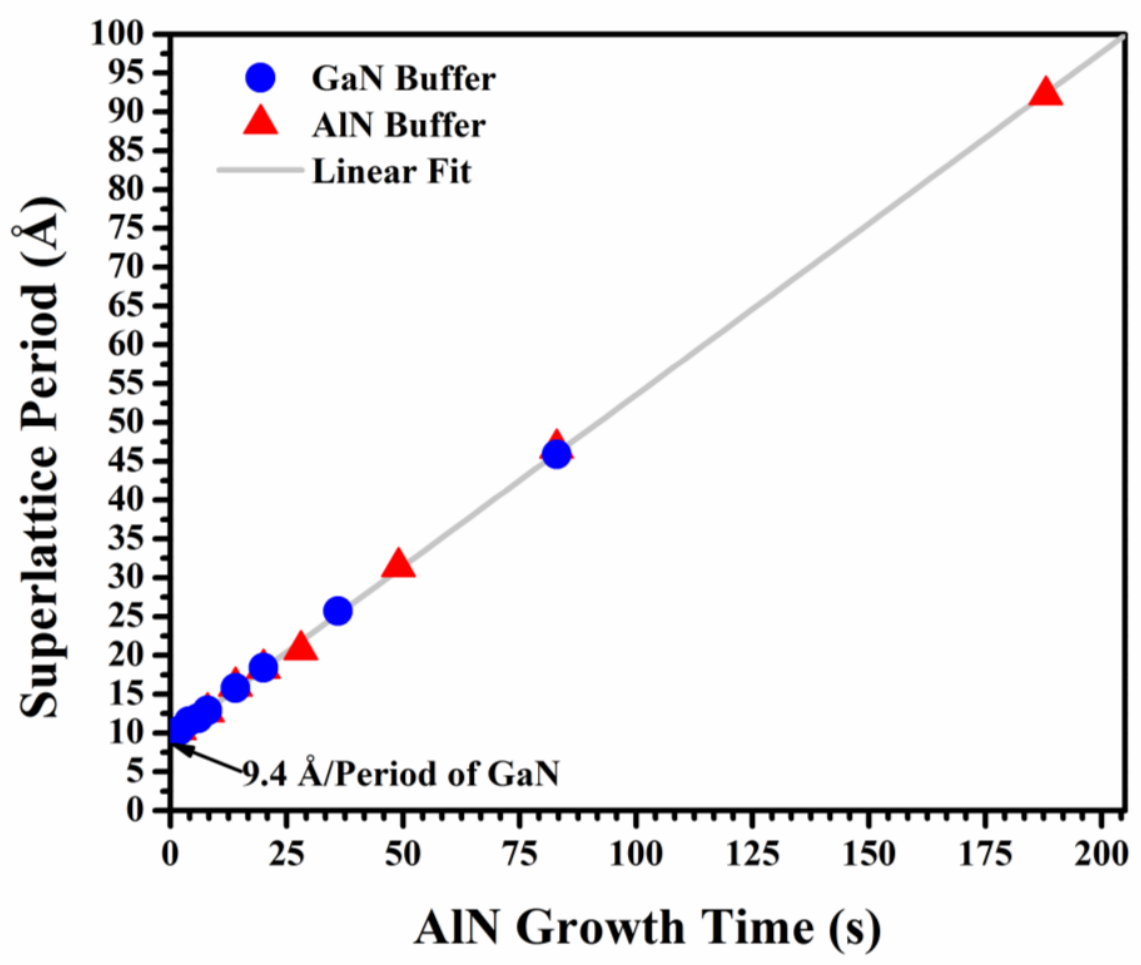

Figure 4-6 - Period of the digital alloys as a function of AlN growth time for alloys grown on GaN and AlN buffer layers.

based on the thickness of each period and the assumption that each period contains approximately $9.4 \AA$ of $\mathrm{GaN}$ as extracted from Figure 4-6 using the following equation:

$$
\text { AlN Mole Fraction }=\frac{\text { Period }-9.4 \AA}{\text { Period }}
$$

The dotted line is the theoretical AlN mole fraction based on the AlN growth rate of $0.45 \AA / \mathrm{s}$. As it can be seen, $\mathrm{Al}_{x} \mathrm{Ga}_{1-x} \mathrm{~N}$ alloys with concentrations raging $x=0.1$ to $x=0.9$ were successfully grown using this digital technique. In order to validate this method of calculating the Al composition in the alloys, Vegard's law was also used. The films in this work exhibited 


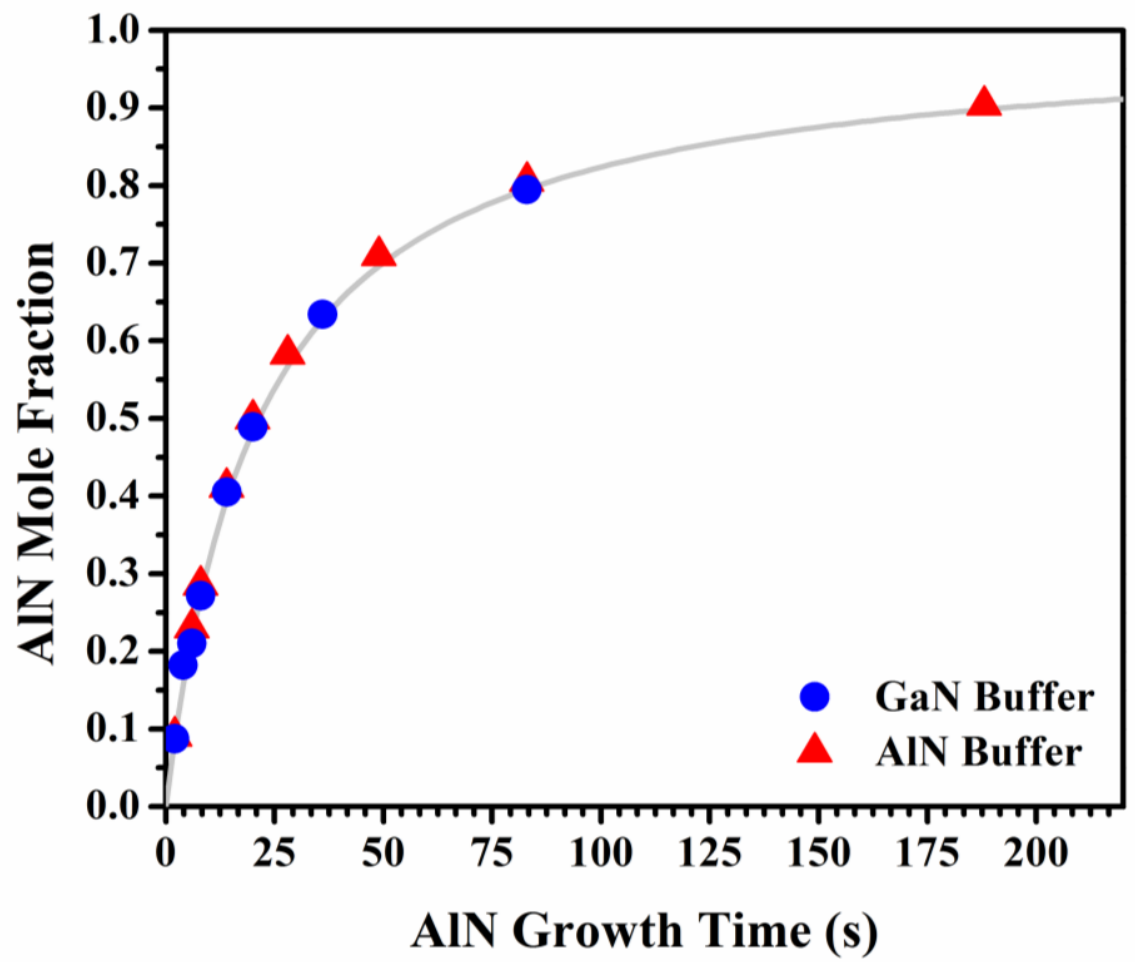

Figure 4-7 - AlN mole fraction of the digital $A l_{\mathrm{x}} G a_{1-\mathrm{x}} N$ film calculated from the periodicity.

considerable biaxial strain as shown in Figure 4-8 and Figure 4-9. In the absence of biaxial strain, the lattice parameter for $\mathrm{Al}_{x} \mathrm{Ga}_{1-x} \mathrm{~N}$ films will vary linearly with the AlN mole fraction according to Vegard's law. This nominally relaxed relationship for $\mathrm{Al}_{x} \mathrm{Ga}_{1-x} \mathrm{~N}$ alloys is shown in Figure 4-8 and Figure 4-9 as the straight line connecting the two star points. However, the digital alloys appear to be under in-plane tensile biaxial strain as they appear to fall on the right side of the nominal line. The dashed lines indicate the change in lattice parameters for an $\mathrm{Al}_{x} \mathrm{Ga}_{1-x} \mathrm{~N}$ film of a specific Al composition, $x$, under biaxial strain. The relationship between the $c$ - and $a$ - lattice parameters is given by the following ${ }^{103}$ :

$$
\frac{c_{S}-c_{o}}{c_{o}}=-2 \frac{S_{13}}{S_{33}} \frac{a_{S}-a_{o}}{a_{o}}
$$

In this equation, $c_{s}$ is the $c$-lattice parameter of the strained film, $c_{o}$ is the nominally relaxed lattice parameter, $a_{s}$ is the $a$-lattice parameter of the strained film, $a_{o}$ is the nominally relaxed $a$ - 
lattice parameter, and $S_{13}$ and $S_{33}$ are the elastic constants for the respective films. The lattice constants and elastic coefficients used in this work were taken from the literature and are shown in Table 4-1. A linear extrapolation of the elastic constants between AlN and GaN was used for the $\mathrm{Al}_{x} \mathrm{Ga}_{1-x} \mathrm{~N}$ films. A comparison of the lattice parameter extracted from the superlattice period and also that from the $a$ - and $c$-lattice parameters is shown in Figure 4-10. It is evident that the two methods of calculating the AlN mole fraction result in similar values. Finally, Energy Dispersive X-Ray Spectroscopy (EDS) was also used to verify the AlN mole fraction on a few representative samples. Figure 4-11 shows that this third technique is also in good agreement with the AlN mole fraction calculated from the periodicity of the superlattice.

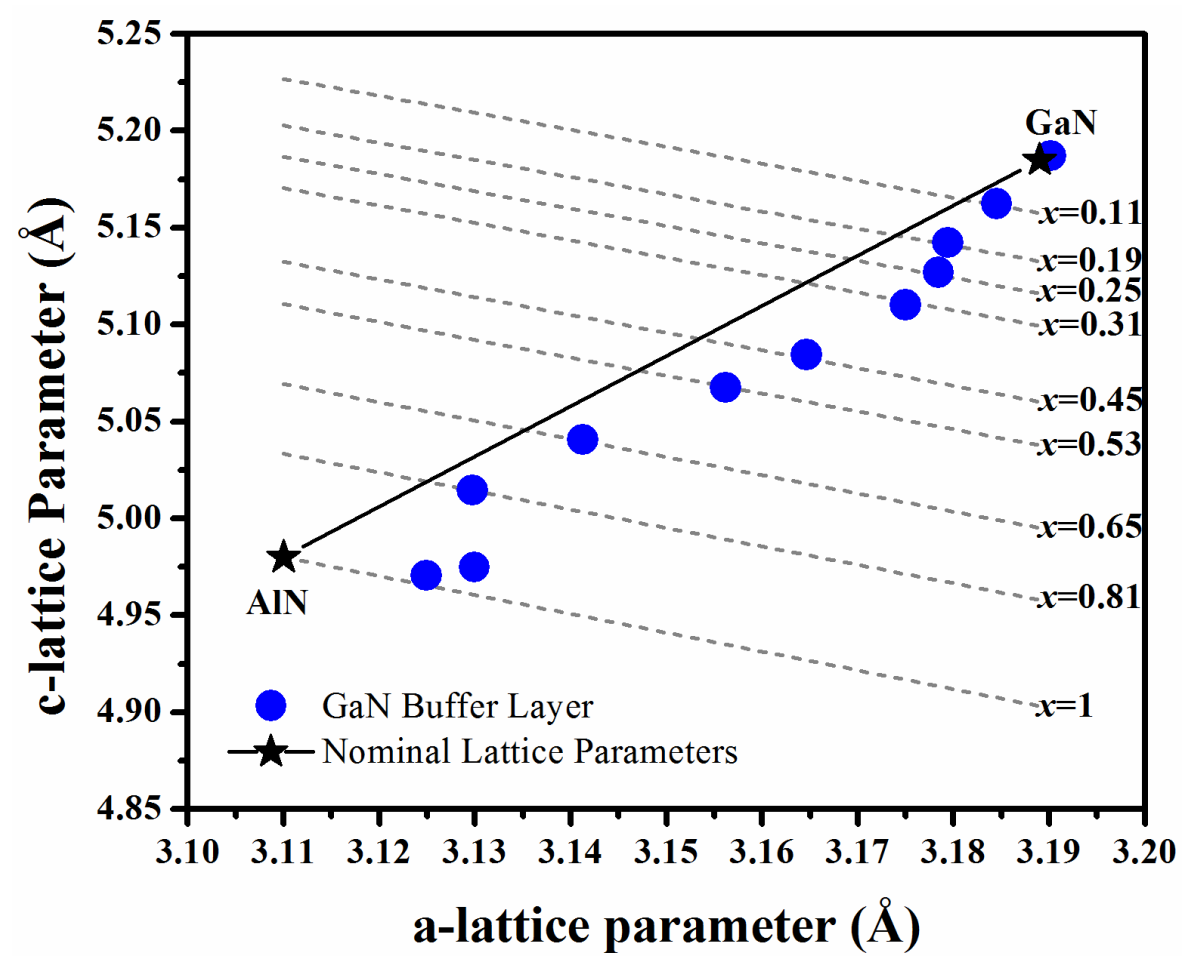

Figure 4-8 - a- and c- lattice parameters for digital alloys grown on GaN buffer layers. 


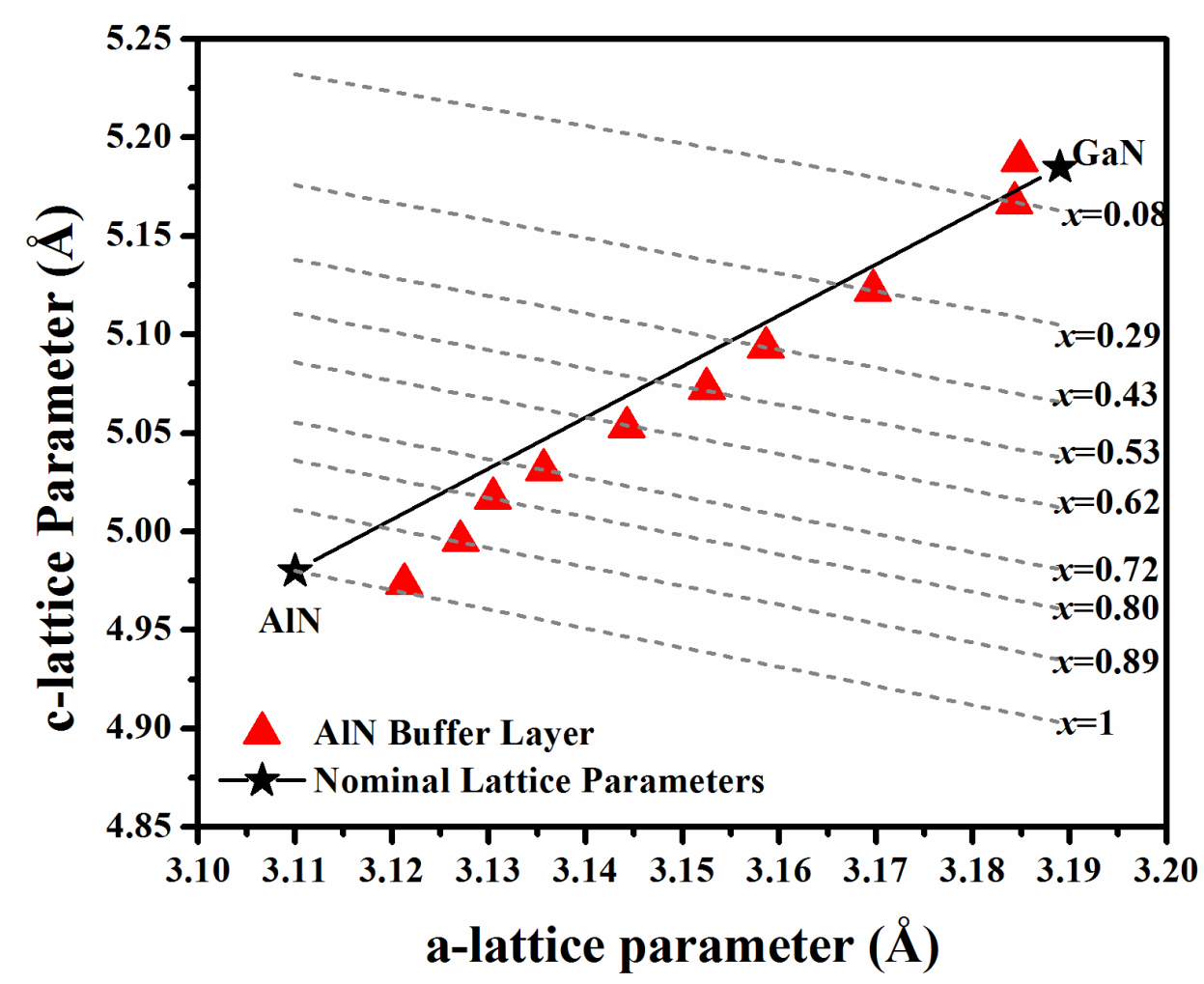

Figure 4-9-a- and c-lattice parameters for digital alloys grown on AlN buffer layers.

Table 4-1 - Lattice parameters and elastic coefficients for GaN ${ }^{43}$ and $\mathrm{AlN}^{2}$

\begin{tabular}{|c|c|c|c|c|}
\hline & $a_{0}(\AA)$ & $c_{0}(\AA)$ & $S_{13}(\mathrm{GPa})$ & $S_{33}(\mathrm{GPa})$ \\
\hline $\mathrm{GaN}$ & 3.189 & 5.185 & 106 & 398 \\
\hline A1N & 3.11 & 4.98 & 120 & 395 \\
\hline
\end{tabular}




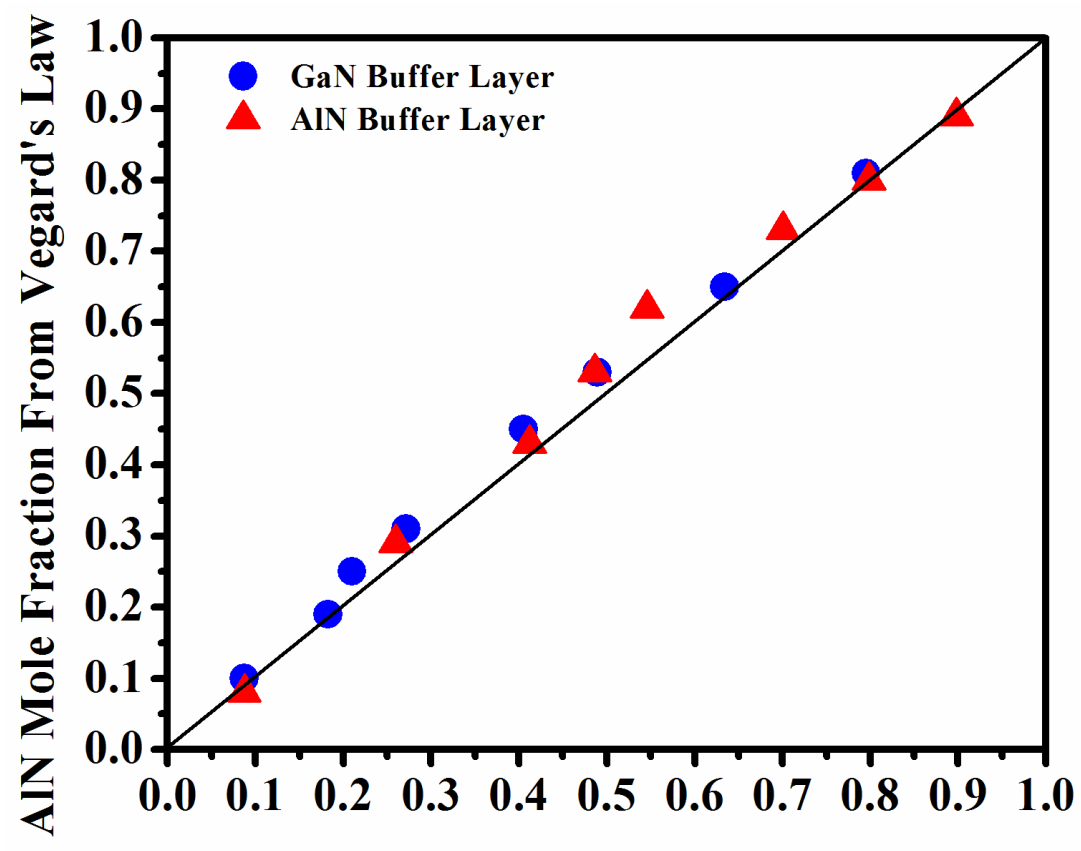

AIN Mole Fraction from period

Figure 4-10 - Comparison of AlN mole fraction calculated from the periodicity and from Vegard's law.

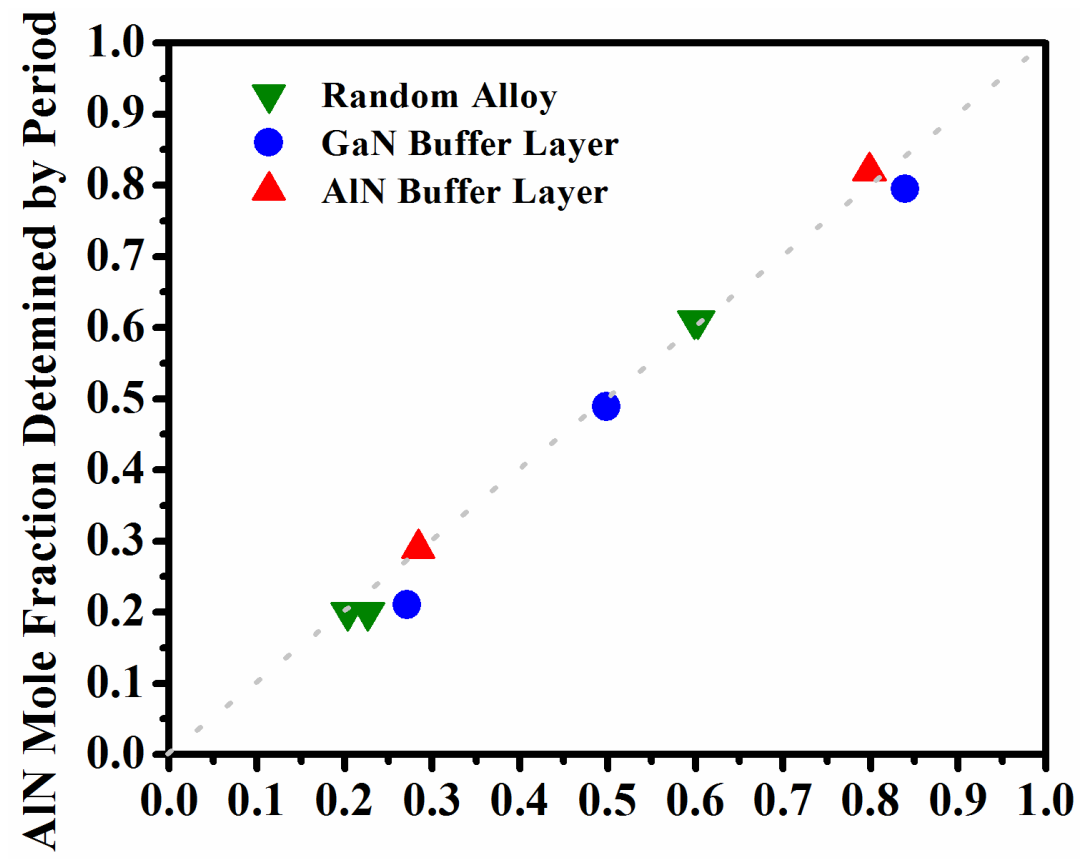

AIN Mole Fraction Determined by EDS

Figure 4-11 - Comparison of AlN mole fraction calculated from the periodicity and EDS measurements. Plot includes digital alloys grown on GaN and AlN buffer layers and also random alloys grown on GaN buffer layers. 
In addition to the series of digital alloys, both $\mathrm{AlN}$ on $\mathrm{AlN}$ and GaN buffer layers were grown, as well as GaN on GaN and AlN buffers at the same growth conditions as the digital alloys. The lattice parameters from these samples were extracted and used as the reference parameters for strain measurements instead of their respective nominal values in order to account for differences in growth methods.

In-plane strain in materials is often measured with respect to the nominal relaxed lattice parameter $\left(a_{0}\right)$ or the underlying buffer layer lattice parameter ( $\left.a_{\text {buffer }}\right)$. The strain with respect to the relaxed film provides insight into the evolution of the strain in the material. However, measuring the strain with respect to the buffer layer allows the conditions for coherent growth, if possible, to be determined. In this work, we will discuss both measurements.

Figure 4-12 contains the strain taken with reference to the nominally relaxed $\mathrm{Al}_{x} \mathrm{Ga}_{1-x} \mathrm{~N}$ film and is given by ${ }^{103}$

$$
\varepsilon_{\mathrm{a}}=\frac{a_{\text {measured }}-a_{0}}{a_{0}}
$$

in which $a_{\mathrm{o}}$ is the nominal lattice parameter and the $a_{\text {measured }}$ is the lattice parameter for the superlattice. The nominally relaxed parameter is calculated using Vegard's law and the measured AlN and GaN lattice parameters grown on each respective buffer layer. The strain profiles on each buffer layer are similar as shown in Figure 4-11. For the digital alloys grown on the GaN buffer layer, the effective digital alloy accumulates an increasing amount of strain up to an AlN mole fraction of 0.3. After which there is a decrease in the stored energy and the lattice constant moves close to the nominal value. Beyond an AlN mole fraction of 0.5, the sign of the strain changes. This is attributed to the fact that for high AlN mole fractions, the AlN in the superlattice dominates the lattice parameter and the measured superlattice parameter is therefore smaller than the nominal value. A similar trend is observed for the digital alloys grown on AIN buffer layers.

Figure 4-13 shows the in-plane strain measured with respect to the buffer layer given by

$$
\varepsilon_{\mathrm{a}}=\frac{a_{\text {measured }}-a_{\text {buffer }}}{a_{\text {buffer }}}
$$


in which $a_{\text {buffer }}$ is the in plane lattice parameter of the buffer layer and the $a_{\text {measured }}$ is the lattice parameter for the superlattice. There is more error in calculating the in-plane lattice parameter of the buffer layer due to the thickness of this layer when compared to the superlattice. As can be seen, $\mathrm{Al}_{x} \mathrm{Ga}_{1-x} \mathrm{~N}$ digital alloys grown on a GaN buffer with an AlN mole fraction from 0 to approximately 0.3 appear coherent $\left(a_{\text {measured }}-a_{\text {buffer }} \approx 0\right)$. Increasing the AlN mole fraction, by increasing the thickness of the AlN layer, beyond 0.3 results in the apparent relaxation of the digital alloy. It appears that at an AIN mole fraction of approximately 0.8 , the AlN in the superlattice dictates the lattice parameter as it has saturated at the strain value for AIN grown on a GaN buffer (no superlattice). For the samples grown on the AlN buffer layer, a similar trend can be observed however, the coherent state is for high Al content alloys. Apparent relaxation occurs for all alloys having an AIN mole fraction less than 0.8. Both set of samples appear to

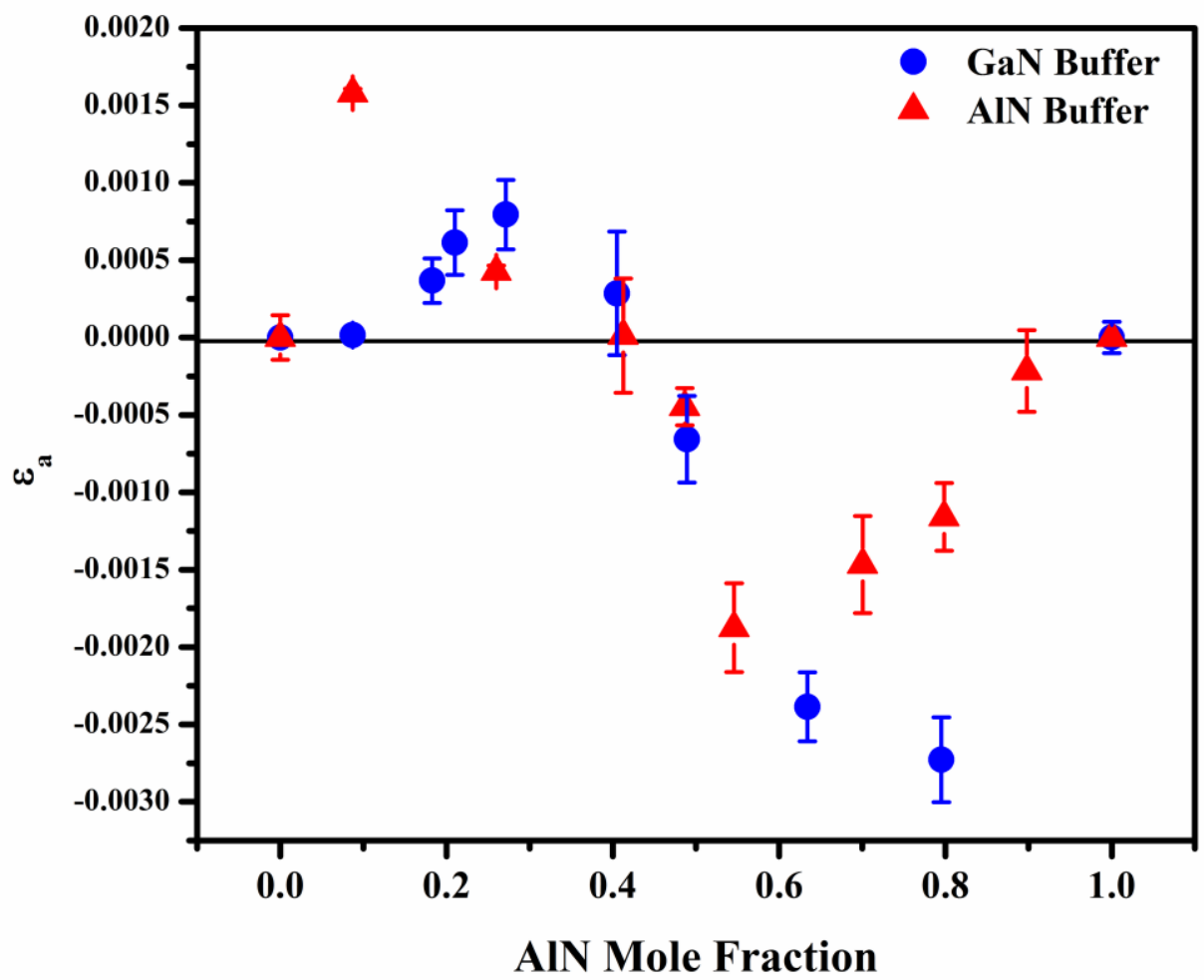

Figure 4-12 - In-plane strain in digital alloys with respect to the nominally relax lattice parameter. 


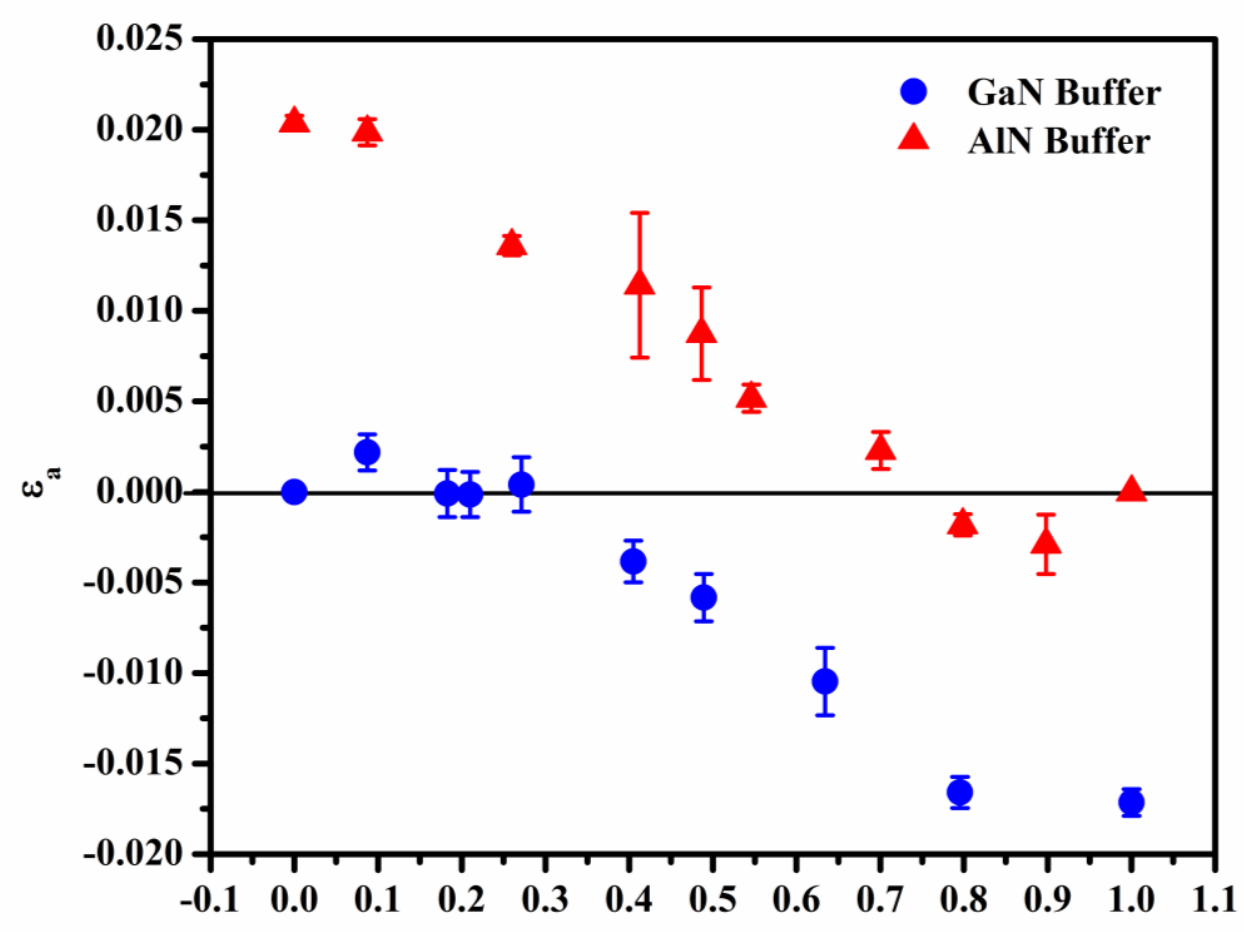

AIN Mole Fraction

Figure 4-13 - In-plane strain in digital alloys with respect to the underlying buffer layer.

saturate at a strain value of approximately 0.02 . This is slightly less than the strain between fully relaxed AlN and GaN ( 0.025) indicating that that even the binary AlN and GaN layers are not fully relaxed as grown under these conditions. There is no large scale cracking observed in these samples and therefore it can be speculated that the majority of strain relaxation occurs due to the surface roughening, surface undulation, or the generation of dislocations or other defects ${ }^{67}$, however the resources to accurately determine the defect density are not available. The RMS surface roughness measured by atomic force microscopy (AFM) over $1 \mu \mathrm{m}^{2}$ areas for several samples is shown in Figure 4-14. The surface roughness does increase as the AlN layer thickness is increased in the superlattice for growth on both buffer layers. While there is a similar trend observed for growth on both buffer layers, the growth on AlN buffer layers roughens more at high AlN mole fractions as compared to the growth on $\mathrm{GaN}$ buffer layers. This roughening may be one possible means of relaxation in the film. It has been reported that the theoretical critical thickness for relaxation of an AlN/GaN superlattice is between $25 \AA$ and $37 \AA^{73}$. In this work, the onset of relaxation begins at the point in which the superlattice begins 


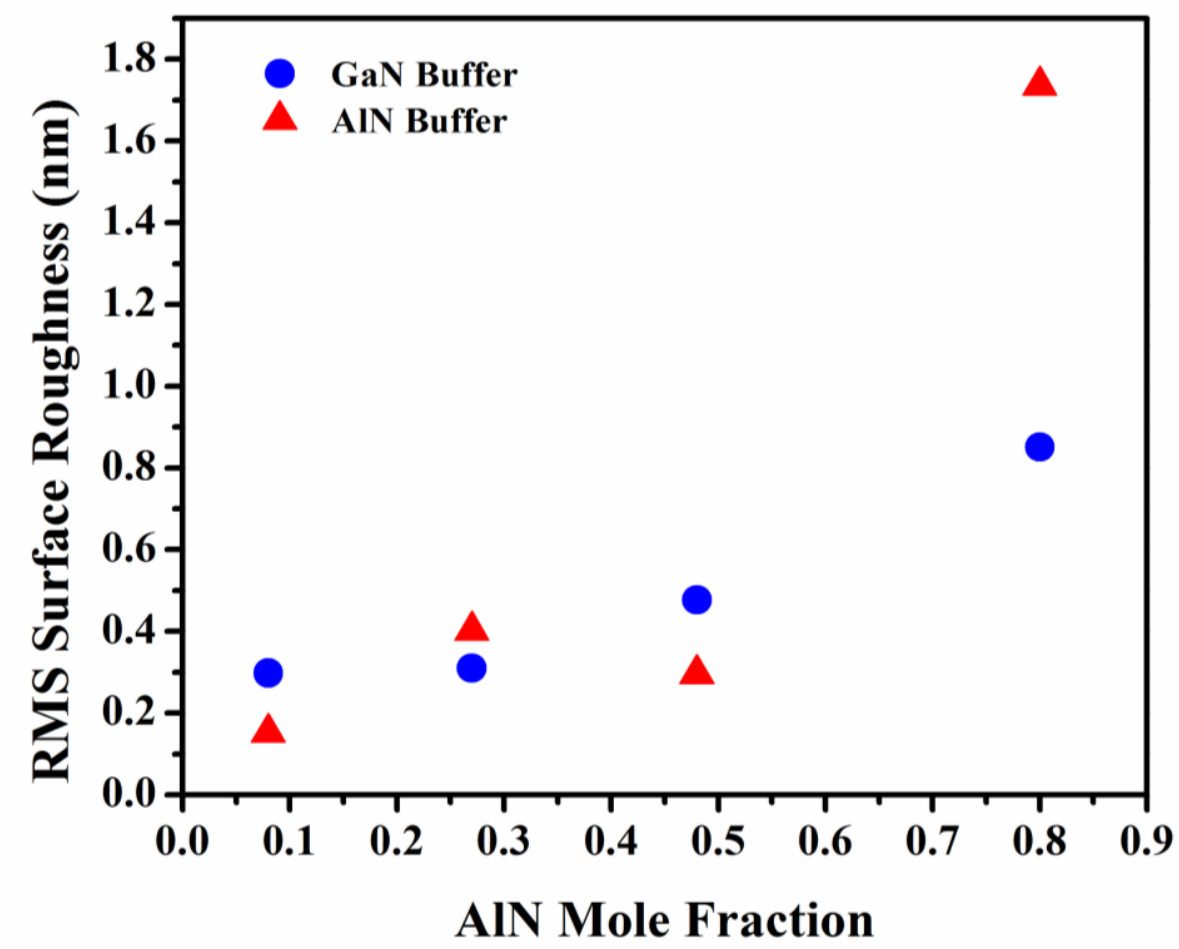

Figure 4-14-RMS surface roughness for digital $A l_{\mathrm{x}} G a_{1-\mathrm{x}} N$ films.

to become incoherent with the buffer layer. For digital alloys grown on $\mathrm{GaN}$ buffer layers, this occurs around an AlN thickness of $3.5 \AA$. This is approximately an order of magnitude thinner than that predicted in the literature, as shown in Figure 4-15 ${ }^{73}$. It should be emphasized that the calculations performed by Bykhovski et al. considered a free standing AlN/GaN superlattice of equal layer thickness with no influence from a buffer layer or substrate. Furthermore, growth kinetics play a large role in strain formation in III-Nitride materials and could also contribute to the discrepancy ${ }^{123}$. The relaxation of the digital alloys does follow a trend similar to that predicted by the theoretical work. Figure 4-16 compares the strain in the digital alloys grown in this work with the strain in other short period superlattices grown via MBE. In this figure it is apparent that the relaxation in both studies is taking place at similar thicknesses.

Using this method we have demonstrated a digital alloy technique for the growth of $\mathrm{Al}_{x} \mathrm{Ga}_{1-x} \mathrm{~N}$ alloys with an AlN mole fraction ranging from approximately 0.1 to 0.9 . The digital technique consists of the growth of a few monolayers of GaN followed by a few monolayers of AlN. The number of AlN monolayers was used to accurately control the AlN mole fraction in 


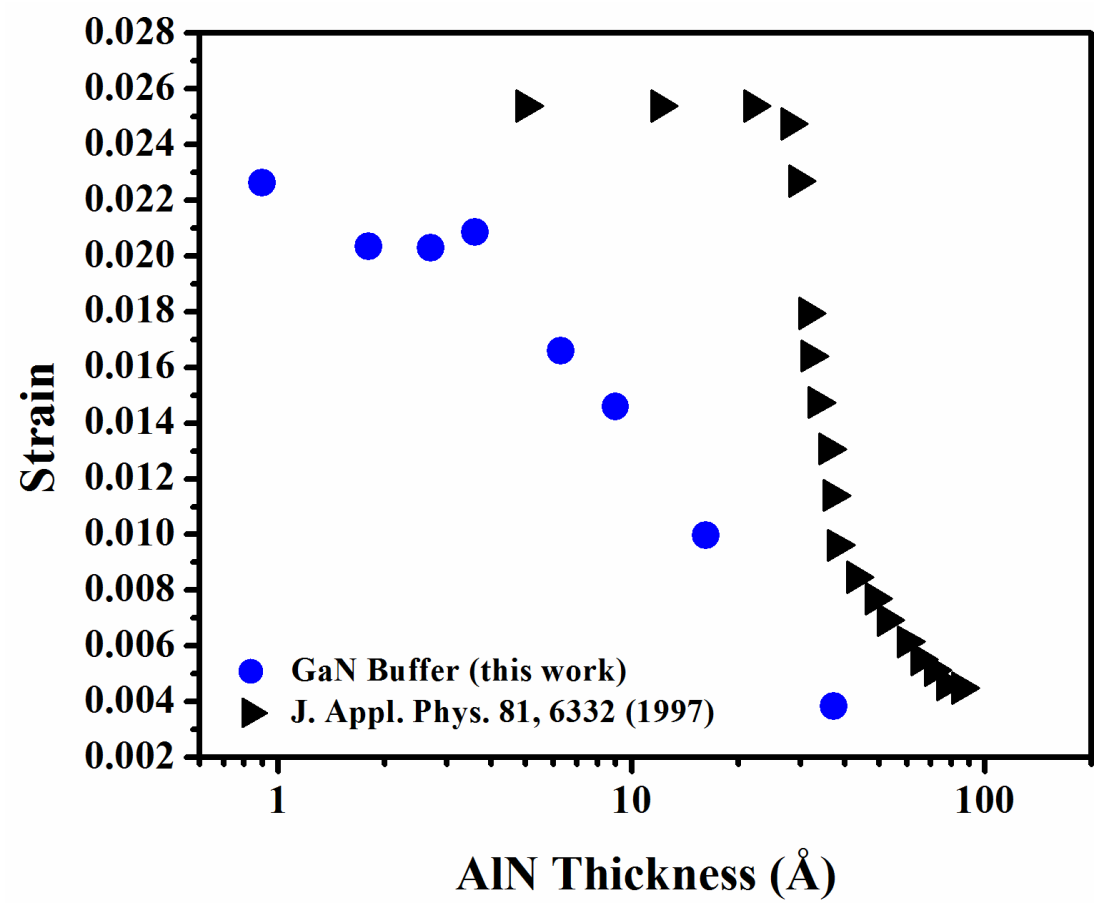

Figure 4-15 - Comparison of strain in digital alloys and theoretical work.

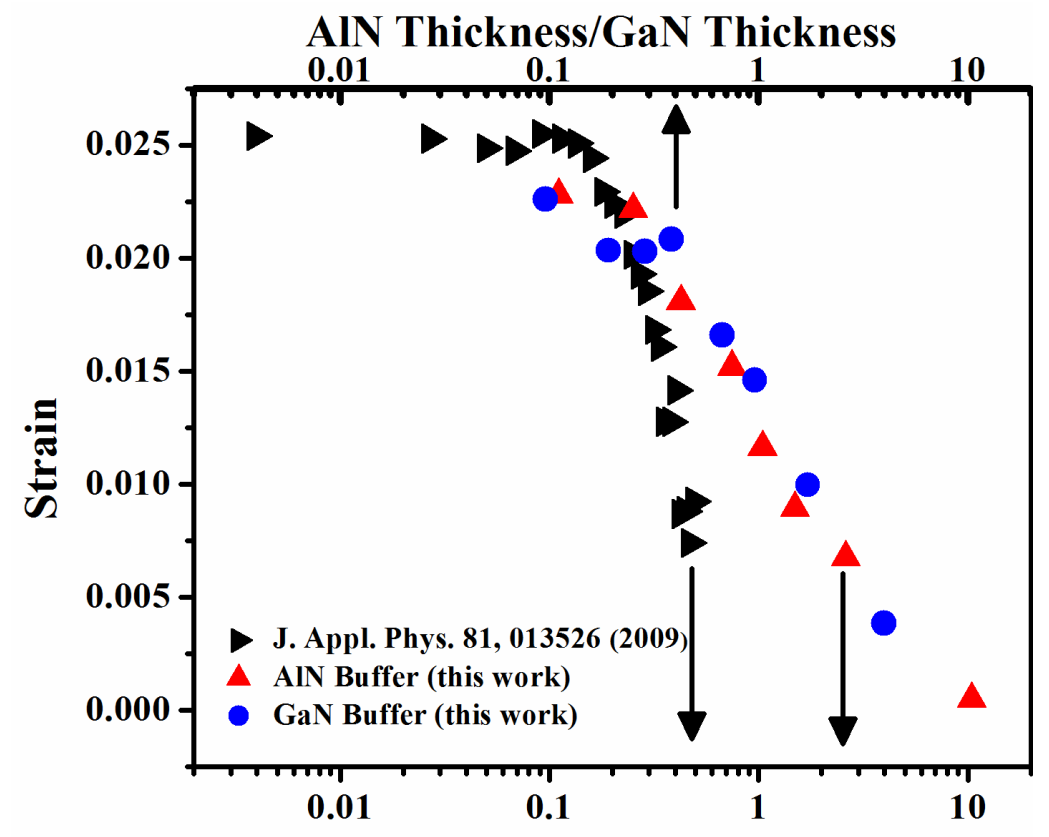

GaN Thickness/AIN Thickness

Figure 4-16 - Comparison of strain in digital alloys and other experimental work on short period superlattices. 
the digital alloy. The superlattice structure and relatively abrupt interfaces were verified using XRD measurements. The strain formation in the digital alloy and the influence of the buffer layer was also investigated. There is initially coherent growth for low Al concentration alloys on $\mathrm{GaN}$ and for high Al concentration alloys on AlN. Relaxation appears to occur for AlN mole fractions greater than approximately 0.3 for digital alloys grown on GaN and for AlN mole fractions less than approximately 0.8 for digital alloys grown on AlN. There is no large scale cracking observed in these structures and therefore the relaxation is attributed to the formation of misfit dislocations and other defects. The thickness of the AIN in the superlattice at which relaxation begins is approximately an order of magnitude less than theoretical predictions. However, it should be noted that lattice mismatch alone is not solely responsible for the relaxation. The thickness before relaxation largely depends on the growth kinetics and could explain many of the large discrepancies in critical thicknesses reported in the literature ${ }^{123}$.

The bandgap of the digital alloys were measured via transmission measurements as described previously. It was experimentally observed that the bandgap of the alloy was slightly higher than the expected bandgap as calculated from the following expression ${ }^{2}$ :

$$
E_{g_{-} A I G a N}=X^{*} E_{g_{-} A I N}+(1-X) * E_{g_{-} G a N}-X b(1-X)
$$

In which $E_{g}$ is the bandgap of the respective materials, $x$ is the AlN mole fraction, and $b$ is the bowing parameter. In this work, the bowing parameter was taken to be $1^{2}$. The bandgap of GaN was $3.4 \mathrm{eV}$ and the bandgap of AlN was $6.2 \mathrm{eV}$. Figure 4-17 shows the bandgap measured for various alloys as compared to the expected value calculated from the equation above. Due to limitations in the white light source, the high aluminum content alloys could not be accurately measured.

For use in optoelectronic devices, it is also important to determine the refractive index of these digital alloys, as well as how it compares to the random alloy equivalent. Unfortunately, there is considerable discrepancy in the refractive index of III-Nitride films reported in the literature. This may be due to different material quality and strain that arises from variations in the growth parameters. In this work, the refractive index was determined from Variable Angle White Light Ellipsometry. From the superlattice measurements, the thickness of the film is well known, and therefore the ellipsometry models as described previously are easily 


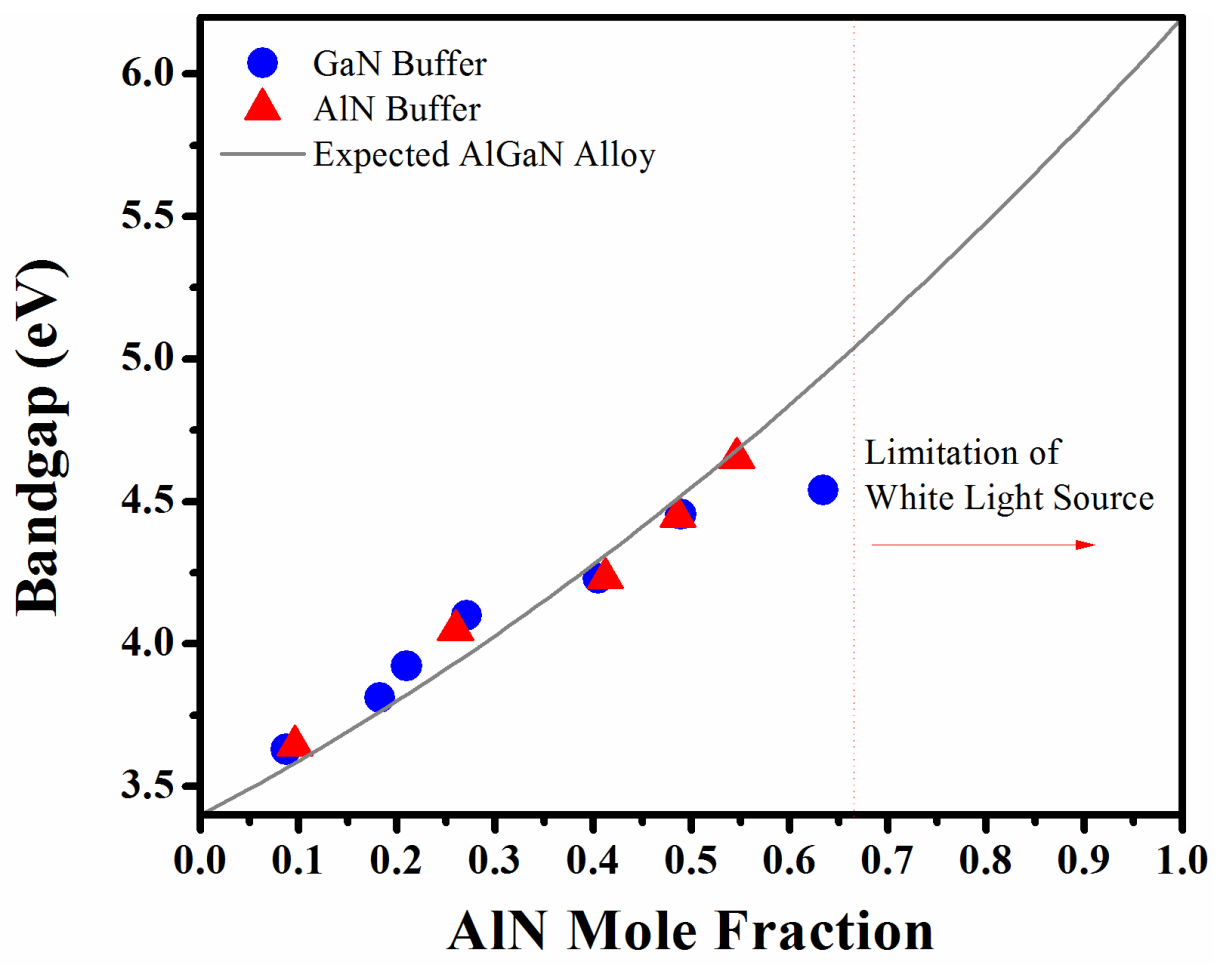

Figure 4-17 - Bandgap of digital alloy as extracted from transmission measurements as a function of the composition.

created. Figure 4-18 shows the measured refractive index of digital alloys grown with a 1s GaN growth time along with values from the literature.

The digital alloys discussed thus far corresponds to a set of samples that was grown with a $1 \mathrm{~s}$ GaN growth time and a variable AlN growth time. Other sets of digital alloys were fabricated by changing the GaN growth time from $1 \mathrm{~s}$ to $2 \mathrm{~s}$ and $3 \mathrm{~s}$. This increases the GaN layer thickness and changes the AlN mole fraction. Figure 4-19 shows how the periodicity varies for sets of digital alloys grown with $1 \mathrm{~s} \mathrm{GaN}, 2 \mathrm{~s} \mathrm{GaN}$, and $3 \mathrm{~s} \mathrm{GaN} \mathrm{growth} \mathrm{times.} \mathrm{All} \mathrm{three} \mathrm{sets}$ exhibit a linear dependence on the AIN growth time as expected. The linear fit of each yield an AlN growth rate of around $0.45 \AA / s$. From the y-intercept of the linear fit we can extract the $\mathrm{GaN}$ thickness grown in each period and therefore the GaN growth rate. Figure 4-20 shows the evolution of the growth rate as a function of the GaN thickness. At the interface it appears approximately $9.4 \AA$ is grown in the first 1 second under the conditions used in this work. However, the growth rate is clearly not $9.4 \AA / \mathrm{s}$ as can be seen by the periodicity shown in 


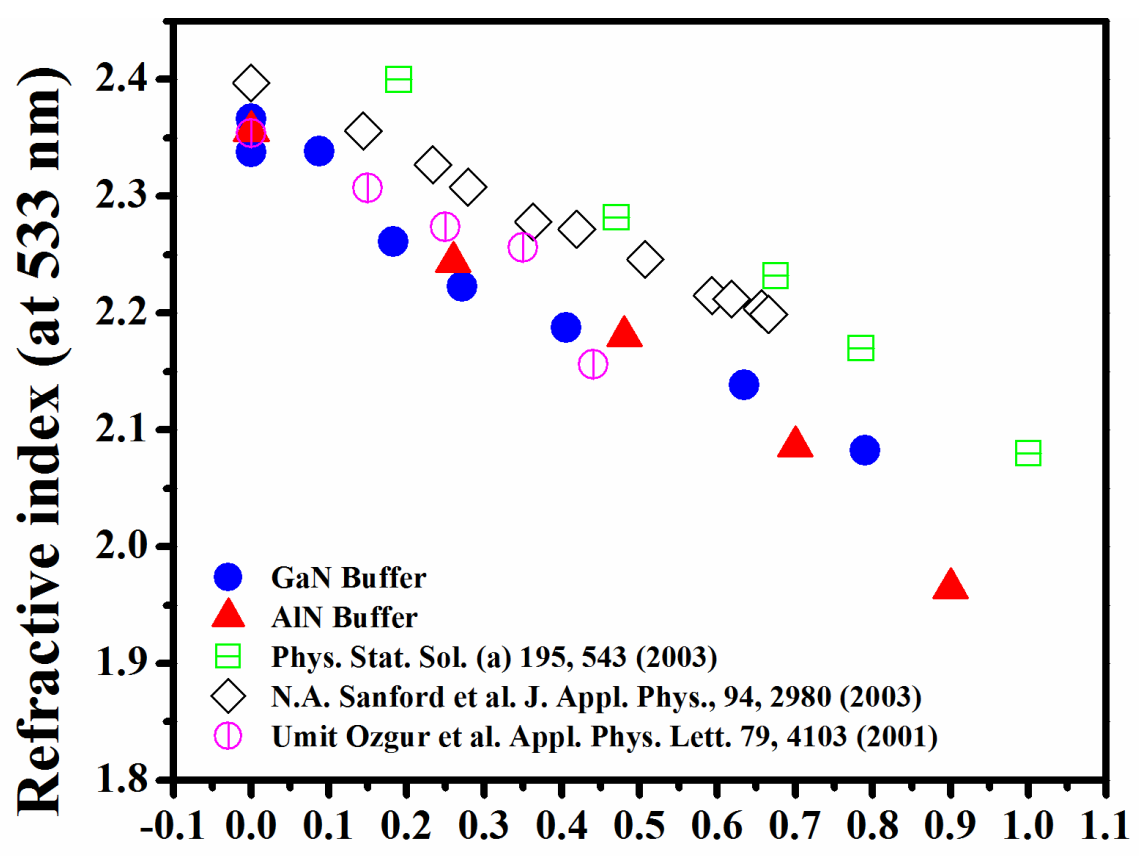

AIN Mole Fraction

Figure 4-18-Refractive index of digital alloys measured from ellipsometry.

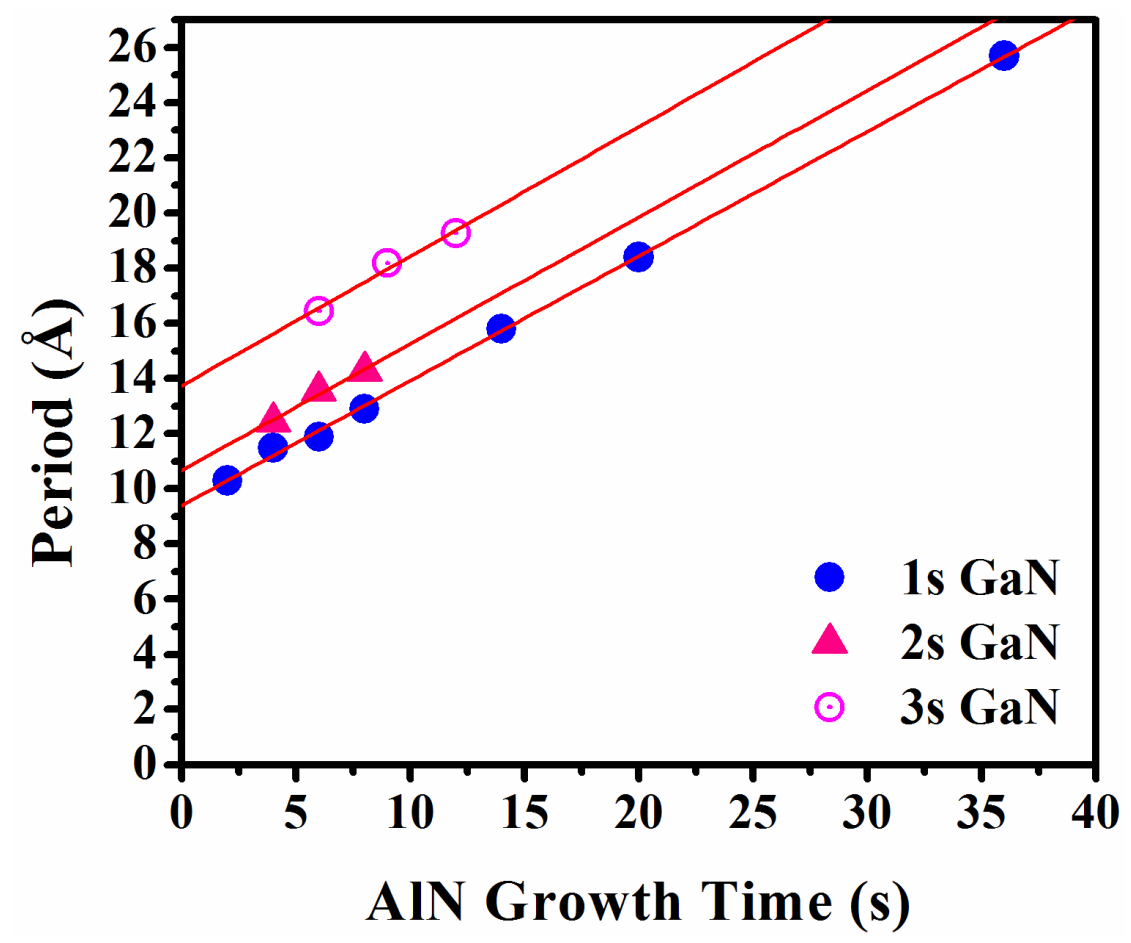

Figure 4-19 - Periodicity of digital alloys with varying GaN growth times. 
Figure 4-19. After approximately $14 \AA$ of GaN growth, the growth rate appears to approach the nominal value for thick films as shown in Figure 4-20. Thick films correspond to layers greater than $400 \mathrm{~nm}$. This indicates that the growth at the interface is almost twice as fast at the nominal growth rate.

Additionally, the digital alloys were grown at different temperatures to investigate the robustness of the growth technique. Figure 4-21 shows four sets of digital alloy samples all grown with a GaN growth time of $1 \mathrm{~s}$ and variable AlN growth time. Samples were grown at $960{ }^{\circ} \mathrm{C}, 1000{ }^{\circ} \mathrm{C}, 1032{ }^{\circ} \mathrm{C}$, and $1040{ }^{\circ} \mathrm{C}$ and the periodicities have been compared. Figure $4-21$ shows there is a considerable change in the periodicity indicating a strong dependence of the superlattice growth rate on growth temperature. This is contrary to the thick film growth rates of AlN and GaN, which have been experimentally verified to be relatively independent of growth temperature over this range.

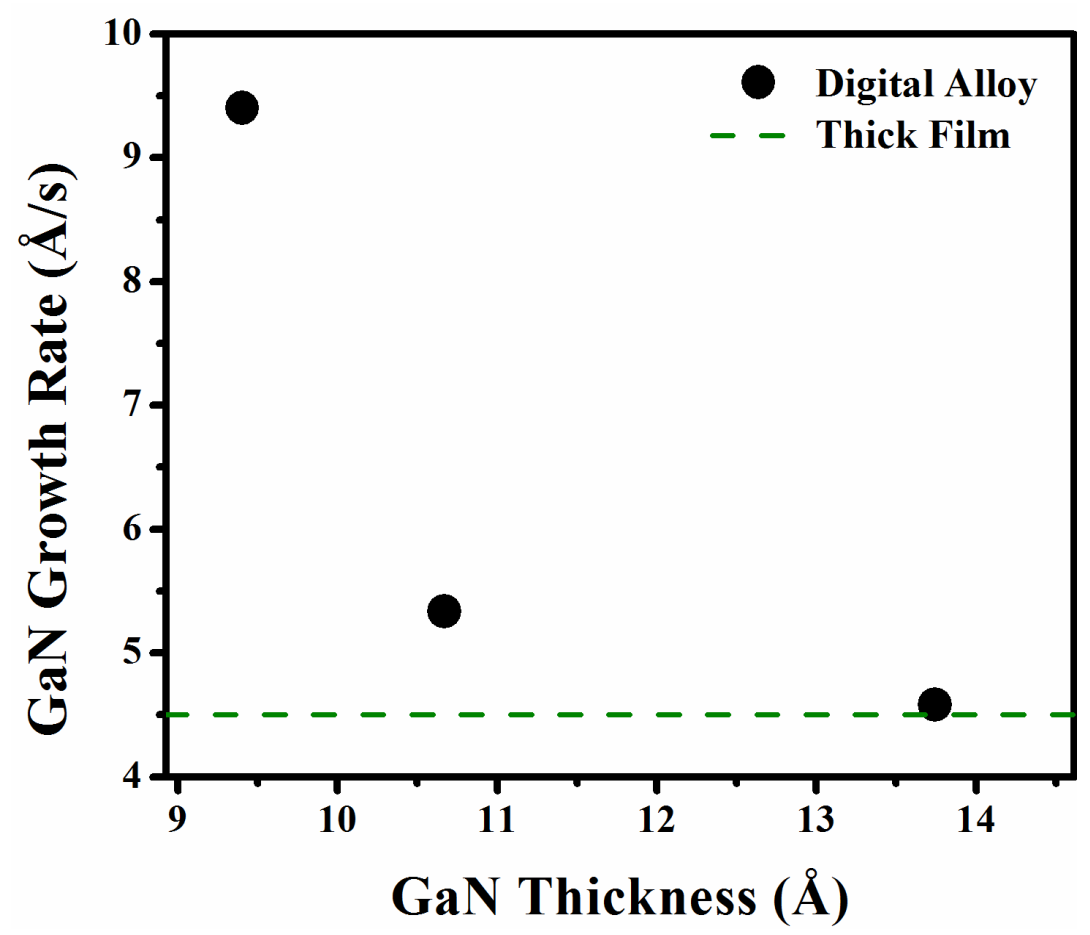

Figure 4-20 - Growth rate of GaN layer in digital alloy as a function of the thickness of the GaN layer. As the thickness increases, the growth rate approaches the nominal value for thick (>500 nm) films. 


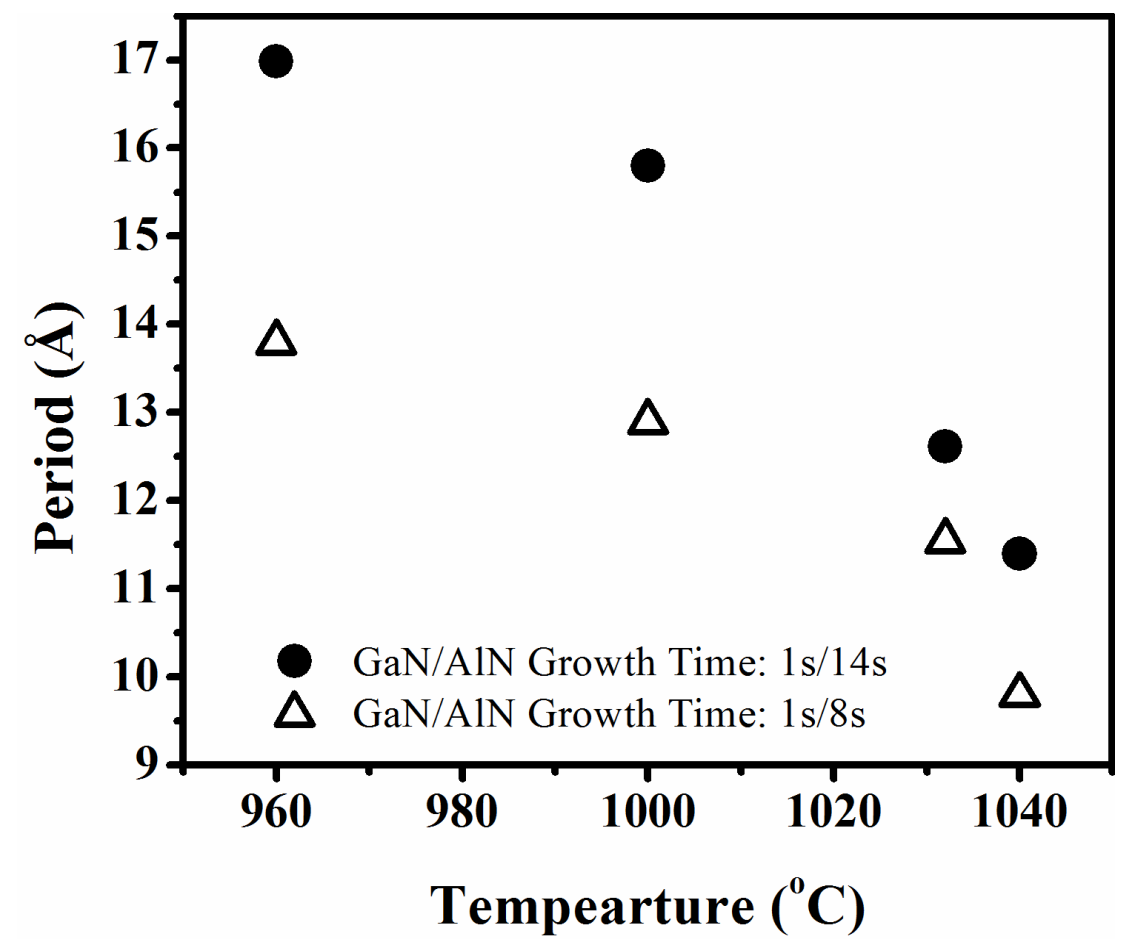

Figure 4-21 - Periodicity of digital alloys grown with a GaN/AlN growth time a 1s/14s and $1 s / 8 s$ at various temperatures. 


\section{H A P T E R $5-$ D IS T R B U T E D B RA G G R E F L E C T O R S}

\subsection{Introduction}

Distributed Bragg Reflectors have been investigated in this work for integration in IIINitride based Light Emitting Diodes (LEDs) based on the structure shown in Figure 1-1 and related devices. In typical III-Nitride based LEDs grown on sapphire substrates, light generated in the active region is emitted from the backside of the device, guided in the sapphire substrate, guided in the GaN slab, or emitted from the top ${ }^{104}$ as shown in Figure 5-1. Only that emission from the top is considered useful for the lighting applications while the rest is loss. In the targeted structure, the DBR is used to reflect light that would be emitted from the backside of the device or trapped in the sapphire substrate, thereby increasing the light extraction efficiency as shown in Figure 5-2. As discussed section 2.4 above, III-Nitride based DBRs are difficult to fabricate due to the large lattice and thermal mismatch between binary materials in this system. In this work, two different techniques have been targeted for the growth of III-Nitride based Distributed Bragg Reflectors (DBRs):

- The use of indium as a surfactant during growth

- The use of a digital alloy growth technique for high aluminum containing alloys

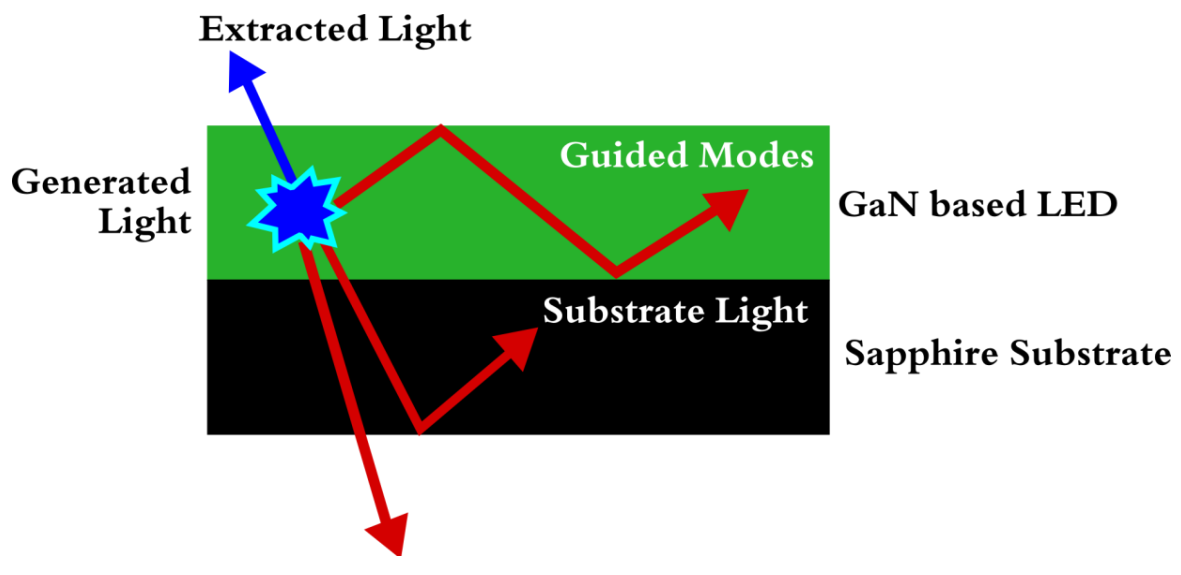

Figure 5-1 - Oversimplified GaN based LED device showing possible light ray paths. Image modified from Ref. 104. 


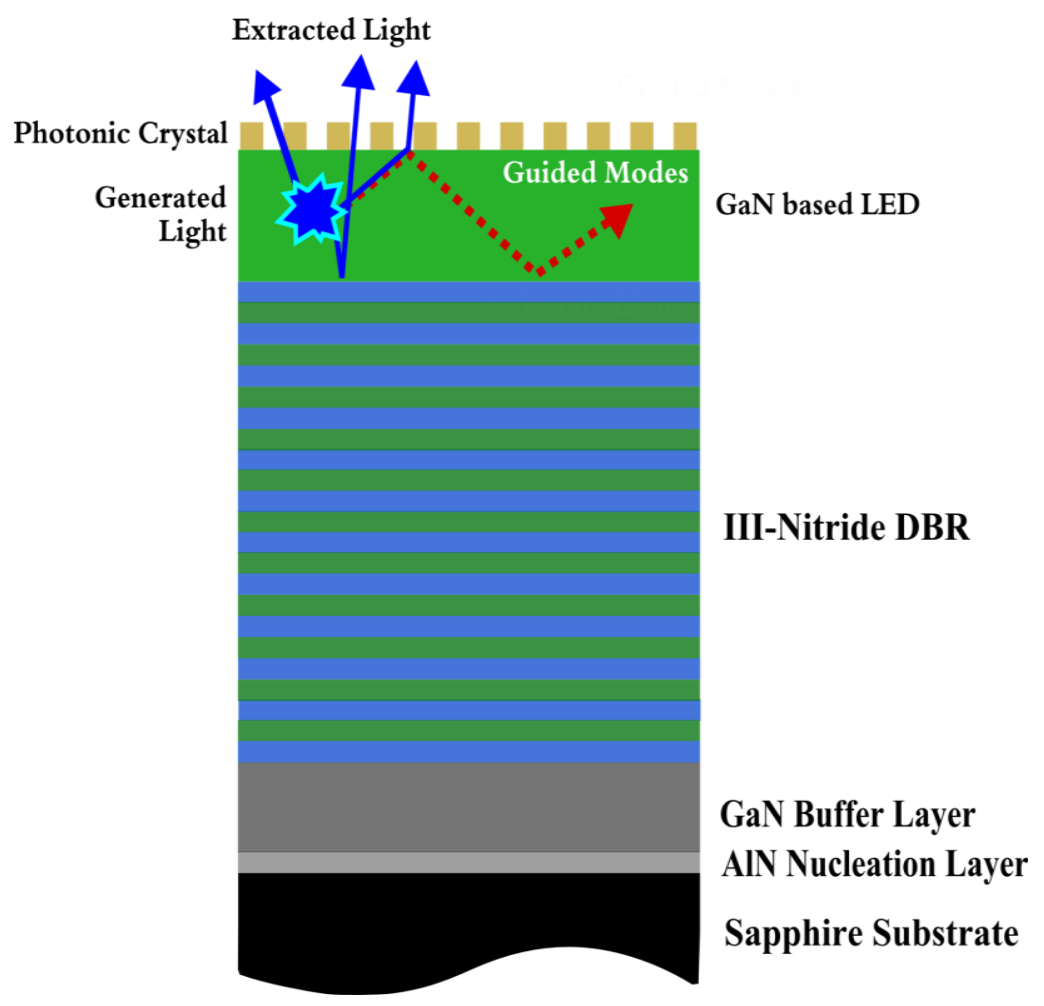

Figure 5-2 - Targeted structure in this work include the incorporation of III-Nitride based DBR under active region of $L E D$.

\subsection{DBR Growth using Indium as a Surfactant}

Indium has been used to improve surface morphology and also crystallinity in various IIINitride alloys ${ }^{57,58,60,61}$. While it is expected that indium will provide similar strain relaxation in larger period structures, such as the DBR, it must be verified. In these larger period superlattice structures, the strain formation and distribution is considerably different than that in the short period superlattices (MQWs) and random $\mathrm{Al}_{x} \mathrm{Ga}_{1-x} \mathrm{~N}$ alloys referenced previously. In short period $\mathrm{AlN} / \mathrm{GaN}$ superlattices, the accumulated strain is distributed more equally between the $\mathrm{AlN}$ and $\mathrm{GaN}$ layers ${ }^{73}$ when compared to larger period superlattices. In random $\mathrm{Al}_{x} \mathrm{Ga}_{1-x} \mathrm{~N}$ alloys, the misfit strain resulting from the lattice mismatch often leads to a composition pulling effect $^{72}$. The inhomogeneous composition distribution caused by this effect influences the strain formation and relaxation. Since AlN is being used instead of $\mathrm{Al}_{x} \mathrm{Ga}_{1-x} \mathrm{~N}$ in this work, there is no compositional distribution to add an additional strain relief mechanism. 
In order to determine the influence of indium as a surfactant on the DBR growth, a structure consisting of only six periods was used as shown in the schematic in Figure 5-3. The primary DBR structure under investigation targeted a center reflectivity wavelength of $465 \mathrm{~nm}$ due to the fact that DBRs reflecting at this wavelength have practical use in blue LEDs for enhanced light extraction or for Resonant Cavity LED fabrication. Additional structures reflecting at $485 \mathrm{~nm}$ and $540 \mathrm{~nm}$, were also investigated. The DBRs were all quarterwavelength, symmetric structures in which the thickness of each layer is $\lambda /\left(4^{*} n\right)$, where $\lambda$ is the center wavelength and $n$ is the refractive index of the material at the center wavelength. For AlN/GaN DBRs targeting reflectivity at $400 \mathrm{~nm}$, no cracking was observed when the structure consisted of five or less periods ${ }^{76}$. As this work primarily targets reflectivity at $465 \mathrm{~nm}$, it can be expected that crack generation might occur in fewer than five periods due to the increased thickness of the layers, but should also occur in a five period structure. In order to isolate the influence of indium on cracking, six period structures were used to exclude, as much as possible, other effects on crack generation. For example, existing defects, such as dislocations and grain boundaries, often serve as the nucleation site for cracks ${ }^{105}$. However, in this work, the resources to accurately define these defect densities are not available. Therefore, the same substrates and growth conditions for the buffer layer were used and it is assumed that the initial dislocation density in each sample is approximately the same.

During the growth of the AlN layer only, trimethylindium was introduced into the reactor as the indium source. Due to the high growth temperature of $\operatorname{AlN}\left(\sim 1000{ }^{\circ} \mathrm{C}\right)$, it was expected that there will be minimal incorporation of the indium into the growth as indium containing alloys are grown at a considerably lower temperature $\left(\sim 700{ }^{\circ} \mathrm{C}\right)$. Under these conditions, it is expected that the indium will largely act as a surfactant. The ratio of timethylindium to trimethylaluminum supplied to the reactor during growth has been varied to investigate the influence of indium. Surface cracking of these structures is visible with the microscope and therefore any changes in the crack density can be clearly observed. Cross sectional SEM images have been used to investigate the layer thickness and also uniformity of the layers. Finally, standard reflectivity measurements were used to verify the reflectivity of the DBRs and any influence of indium on the structures. The reflectivity measurements were performed at normal incidence using a deuterium white light source as described in Chapter 3. 


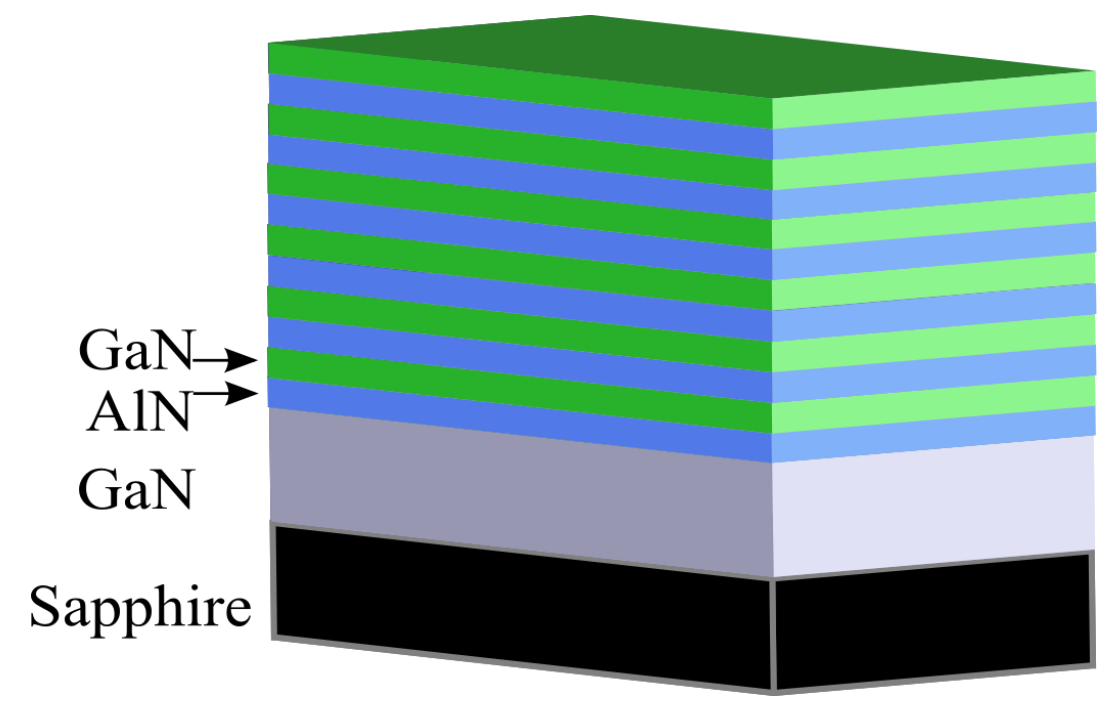

Figure 5-3 - Schematic of AlN/GaN DBR structure.

All materials used in this work were grown via MOVPE in the Aixtron 200/4 RF-S horizontal reactor ${ }^{106,107,108}$. An approximately $2 \mu \mathrm{m}$ thick GaN buffer layer grown on $c$-plane sapphire with a 0.3 degree miscut toward the $a$-axis was used as the substrate for the DBR growth. Trimethylgallium (TMGa), Trimethylaluminum (TMAl), and ammonia $\left(\mathrm{NH}_{3}\right)$ were used as the Ga, Al, and N precursors, respectively. Trimethylindium (TMIn) was introduced into the reactor as the indium source during the growth of the AlN layer only to investigate its influence on cracking. The ratio of the TMIn to TMAl flow rate into the reactor was varied from 0 to 0.6. The growth temperature for the $\mathrm{AlN}$ and $\mathrm{GaN}$ layers were $1000{ }^{\circ} \mathrm{C}$ and $980{ }^{\circ} \mathrm{C}$ respectively and the V/III ratio for the $\mathrm{AlN}$ and GaN layers were 1100 and 700 respectively. Under these conditions the growth rates for the GaN and AIN layers were approximately 28 $\mathrm{nm} / \mathrm{min}$ and $10 \mathrm{~nm} / \mathrm{min}$ respectively.

Quantifying the degree of cracking in thin films is difficult and not commonly reported in the literature. The average distance between cracks has been one metric ${ }^{109}$, but in general, optical microscope images are simply used to visualize the change in crack formation or density. In this work, an image processing technique was employed to quantify the degree of cracking in 
the DBR structures and to investigate the influence of indium. Specifically, Nomarski microscope images, as shown in Figure 5-4a, were taken over at least 50\% of the sample surface. The MATLAB software program was used to convert the original color image to a binary image with the cracks being white pixels and the sample surface represented by black. The contrast between the crack and the sample surface in the original picture is often not enough to simply convert it to a binary image and have an accurate representation of the surface. Any surface roughness or variation in lighting can result in "noise" in the binary image. Therefore, in this work, the cracks were identified by setting a threshold for the number of white pixels in a row that represented a crack. After converting to a binary image, conventional edge detection using built in MATLAB functions was employed to locate the edges of the cracks as shown in Figure 5-4b. From this image, the average crack length per square millimeter was extracted. The total number of white pixels in this figure was divided by two and converted to millimeters to yield the approximate length of the all the cracks in the image area. This number was normalized by the area of each image. All cracking in the DBR structures exhibited a network similar to that shown in Figure 5-4a. The network consisted of a well defined pattern with intersections

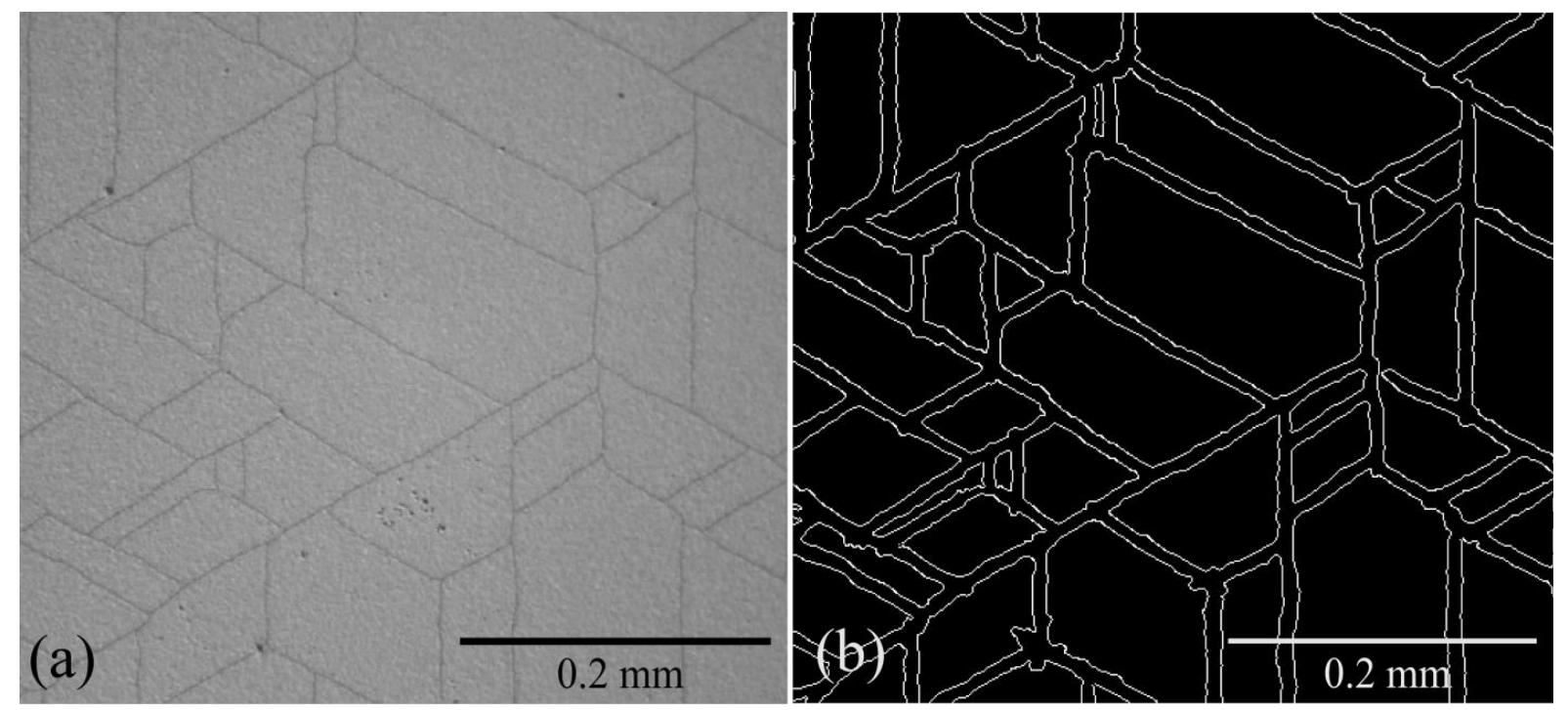

Figure 5-4 - (a) Original microscope image of AlN/GaN DBR and (b) corresponding binary image. 
occurring at $60^{\circ}$ or $120^{\circ}$. For the most part, cracks began or terminated at the intersection with another crack. The MATLAB code for the image processing can be found in Appendix D.

In addition to optical microscope images, cross sectional Scanning Electron Microscopy (SEM) images using a Hitachi S4700 system were used to verify the structure uniformity and layer thickness of the samples. Reflectivity measurements were also and were used as a metric of the DBR's large scale integrity.

Figure 5-5 contains an SEM image of a representative DBR showing all six periods. The lighter and darker layers correspond to $\mathrm{GaN}$ and $\mathrm{AlN}$ respectively. This sample was grown with zero $\mu \mathrm{mol} / \mathrm{min}$ of TMIn. The only observable difference in the cross sectional SEM images of samples grown with and without indium are the density of cracks, therefore the image shown in Figure 5-5 is representative of all samples used in this work. The layer thicknesses were measured from the SEM images and also from white light ellipsometry measurements using a J.A. Woollam M-2000U Variable Angle Spectroscopic Ellipsometer. The difference between the measured thickness and the targeted thickness was less than 5\% and can be attributed to error in the measurement method. Figure 5-6 contains the reflectivity of representative samples exhibiting approximately $60 \%$ reflectivity at $465 \mathrm{~nm}, 485 \mathrm{~nm}$, and $540 \mathrm{~nm}$. Due to limited number of periods, the reflectivity is not characterized by a flat top band width. The simulated value shown was obtained using transmission matrix method ${ }^{110}$ for ideal quarter wavelength DBRs as follows:

$$
\begin{gathered}
t=\frac{\lambda}{4} \\
{\left[\frac{B}{C}\right]=\prod_{r=1}^{q}\left[\begin{array}{cc}
\cos (t) & \frac{i \sin (t)}{n} \\
i n \sin (t) & \cos (t)
\end{array}\right]\left[\begin{array}{c}
1 \\
n_{\text {subs }}
\end{array}\right]} \\
Y=\frac{C}{B} \\
R=\left(\frac{1-Y}{1+Y}\right)\left(\frac{1-Y}{1+Y}\right)
\end{gathered}
$$

In these equations, $t$ is the film thickness, $n$ is the film refractive index, and $R$ is the reflectivity. The refractive indices used in the simulations were experimentally measured using white light ellipsometry measurements. The amount of indium introduced during the AlN growth did not 


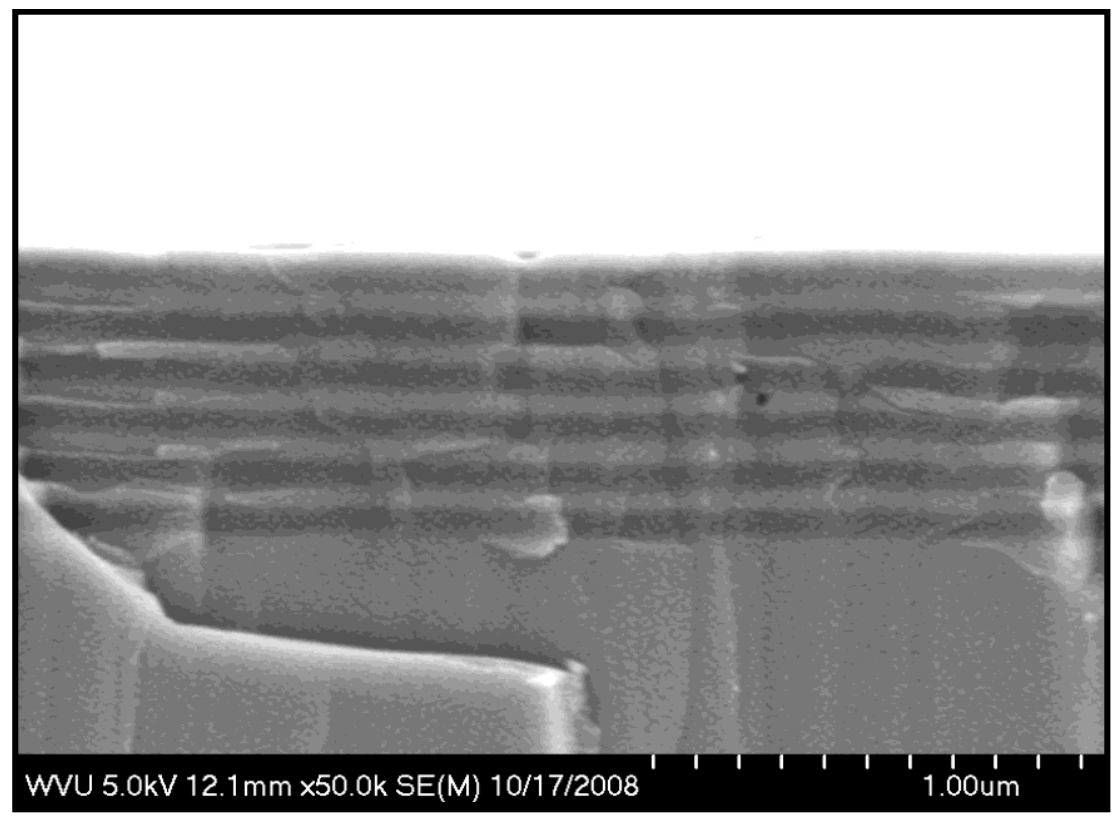

Figure 5-5 - Cross Ssection SEM image of six period AlN/GaN DBR.

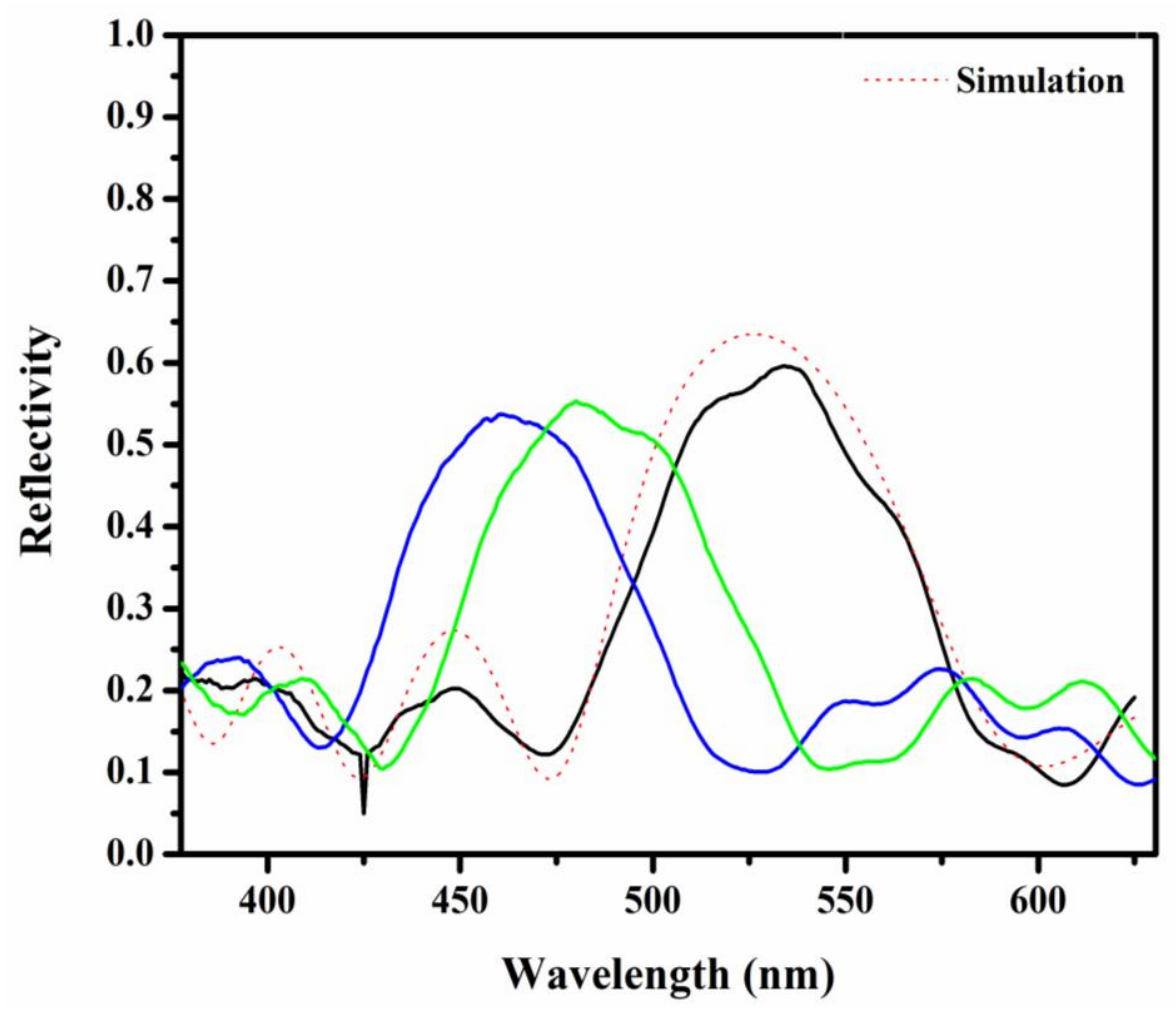

Figure 5-6 - Reflectivity spectra of three different six period AlN/GaN DBR structures. 
have any observable effect on the reflectivity measurements and all measured spectra were statistically identical. Similarly, the amount of cracking in the structures did not produce any observable changes in the reflectivity spectra. The simulation results have also been compared with direct numerical calculations using MEEP, a free finite-difference time-domain (FDTD) simulation software package developed at Massachusetts Institute of Technology. The two methods showed similar, if not identical, results and therefore simulations based on the transmission matrix method were preferred due to its efficiency.

Figure 5-7 shows the crack length per square millimeter as a function of the ratio of the TMIn to TMAl flow rate used during the DBR growth. Each point is the average of images taken randomly over approximately $50 \%$ of the sample surface. It can been seen in this figure that each samples exhibits a similar standard deviation in cracking across the sample as represented by the error bars. During MOVPE several gradients, including temperature and gas flow, influence growth and often result in a thickness difference across the wafer between 3 $25 \%^{111,112}$. As a result, the variation in cracking around $10 \%$ across the surface of the wafer as shown in Figure 5-7 is considered reasonable and can be attributed these gradients. As can also be observed from this graph, the indium significantly influences the crack formation with an apparent minimum around a ratio of 0.3 . Introducing more indium into the system only increases the total crack length. Despite the differences in bi-layer thickness between DBRs reflecting at $460 \mathrm{~nm}, 485 \mathrm{~nm}$, and $540 \mathrm{~nm}$, the influences of indium during growth is consistent as seen in Figure 5-7. These results indicate that under certain growth conditions, the strain in the DBRs is minimized or at least partially accommodated such that the crack formation is reduced by approximately half. At the high growth temperatures used for AlN and GaN, it was anticipated that the indium incorporation would be minimal due to the high desorption rate of indium. Several samples were measured via Secondary Ion Mass Spectroscopy (SIMS) and show an indium incorporation ranging from $5 \times 10^{17}$ to $10^{18}$ atoms $/ \mathrm{cm}^{3}$, even for the highest TMIn flow rate under investigation, as shown in Figure 5-8. This small, but measureable, amount of indium appears to accumulate at the interfaces between the AlN and GaN layers. This work focuses on six period DBRs because it is a complete study in which one growth condition (i.e. indium flow rates) has been investigated. It should be noted however, that using the growth conditions outlined previously, a few samples with a larger number of periods (18) were grown and similar trends in the cracking were observed as illustrated in Figure 5-7. The investigation 


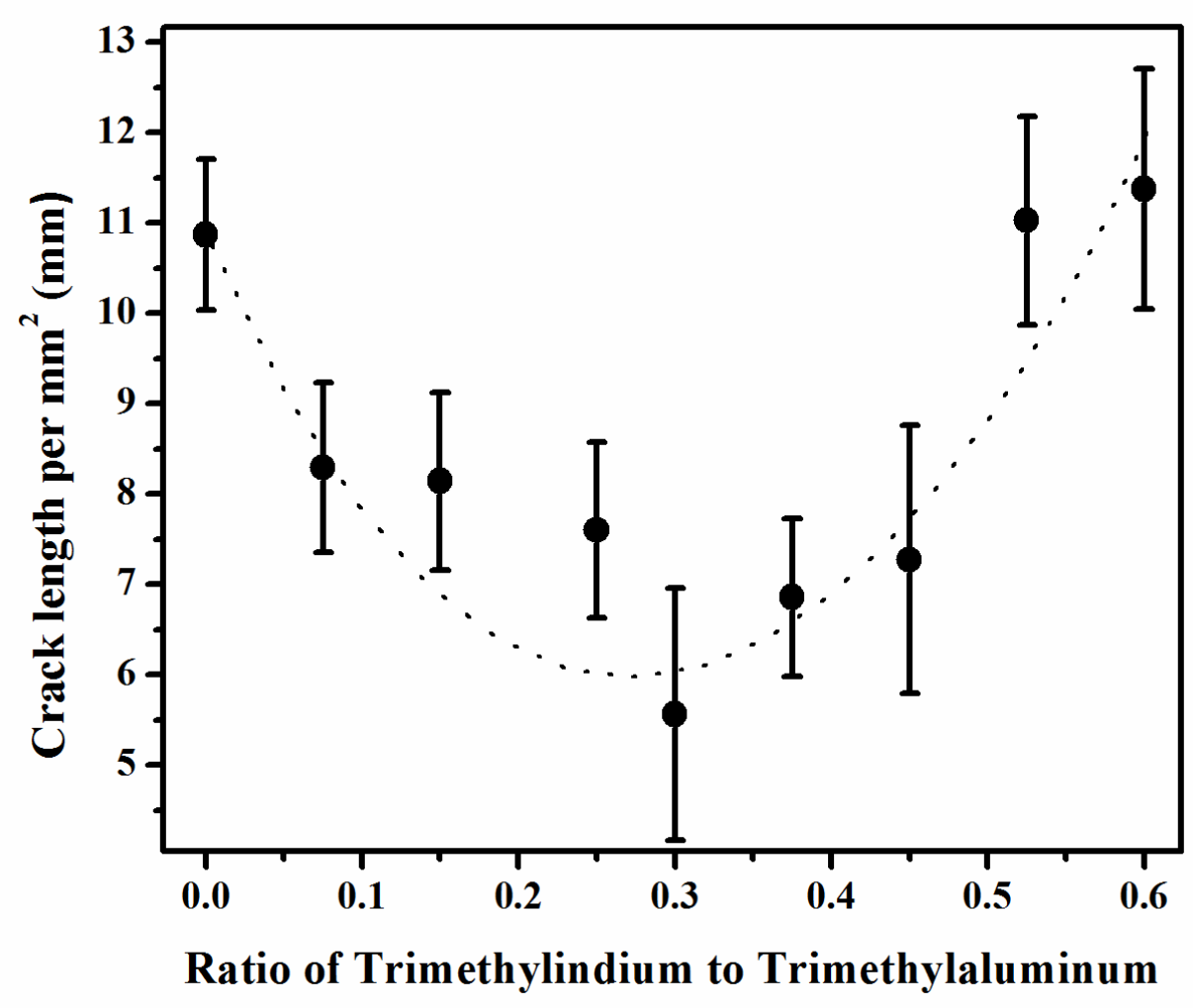

Figure 5-7 - Crack length per square millimeter as a function of the Trimethylindium supplied during growth.

of the 18 period structures is not as comprehensive and was used only to verify that the results presented in this manuscript were transferable to structures with a larger number of periods

Several explanations to the influence of indium in III-Nitride growth has been reported and is generally aimed toward $\mathrm{GaN}$ growth ${ }^{58,60,61,113,114}$. First principle density function calculations have shown that under certain conditions, indium acts as a surfactant ${ }^{113,114}$. In GaN growth, an indium-adlayer on the film surface allows for enhanced diffusion of nitrogen species and is not expected to be incorporated into the growth. While experimental investigations have reported reduced surface roughness due to surfactant effects, the improvement in the crystal quality has been attributed to the incorporation of indium in lattice defect sites and to solution hardening effects ${ }^{57,58}$. Reports regarding the growth of AlN using indium have mainly attributed the influence of indium to surfactant effects which promote a change in the growth kinetics by increasing the diffusion length of the aluminum species ${ }^{60,61,115}$. The increased diffusivity has resulted in improved surface roughness ${ }^{60,61}$ and spiral step flow growth around screw 


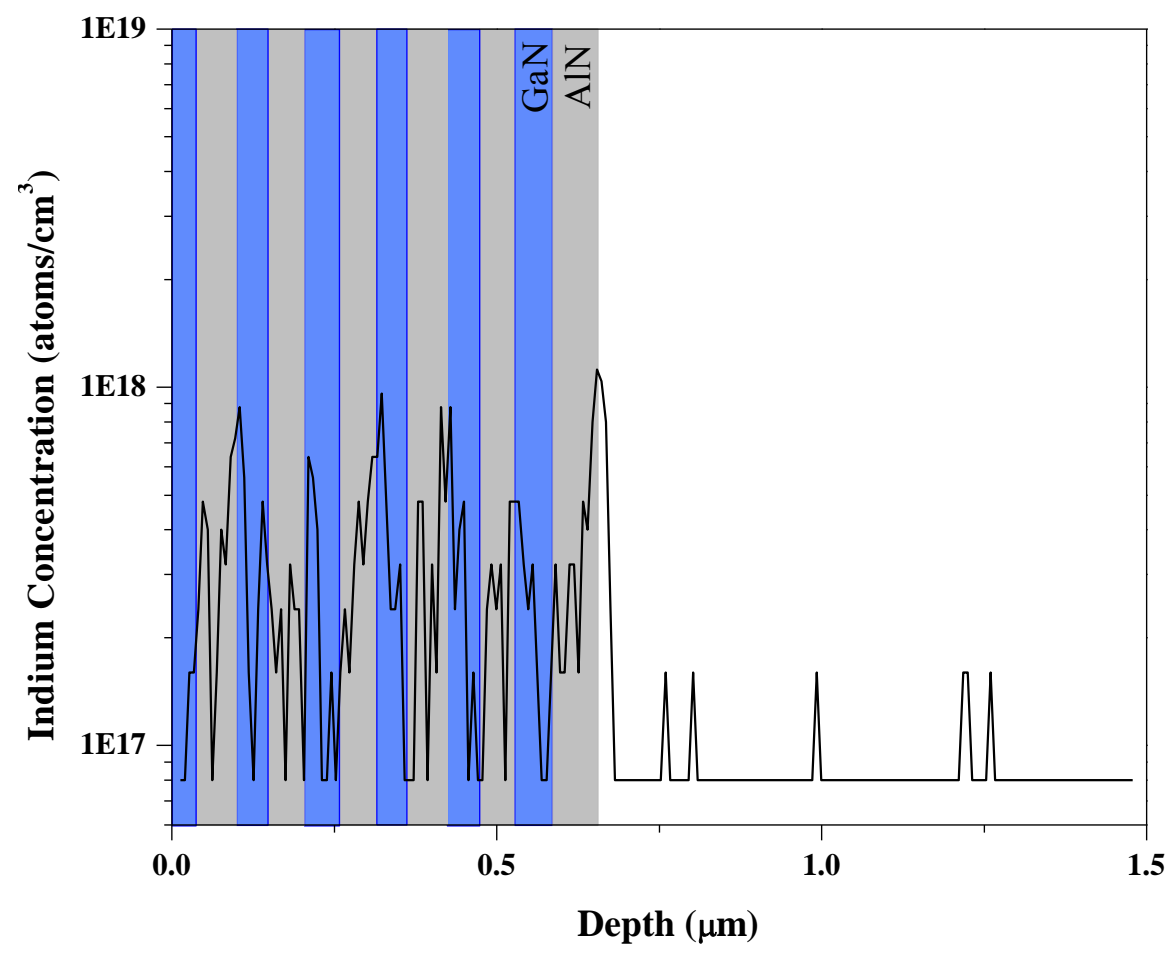

Figure 5-8 - SIMs mesurement of indium incorporation in AlN/GaN DBR.

dislocations promoting a wavy surface morphology to reduce elastic strain ${ }^{60}$. In random $\mathrm{Al}_{\mathrm{x}} \mathrm{Ga}_{1}$ ${ }_{\mathrm{x}} \mathrm{N}$ alloys, crack free layers have been achieved using indium during growth ${ }^{116}$, however the growth kinetics in ternary alloys are different than those in the growth of binary alloys. The sticking coefficients of $\mathrm{Ga}$ and $\mathrm{Al}$ species are considerably different and therefore require different optimal growth conditions ${ }^{41}$. As a result, the role of indium in the growth of random $\mathrm{Al}_{\mathrm{x}} \mathrm{Ga}_{1-\mathrm{x}} \mathrm{N}$ alloys is not necessarily be the same as that in $\mathrm{GaN}$ or $\mathrm{AlN}$ growth.

It is therefore difficult to speculate whether the reasons governing the effects reported in this paper are due to a change in growth kinetics or due to effects produced by the small incorporation of indium atoms. The use of indium during the growth of AlN/GaN DBRs has enabled a means to partially accommodate the strain in the structure and allow for reduced crack formation. Further investigation into the material properties are required to provide a comprehensive explanation. The increase in cracking with large indium flow rates appears to be in agreement with other reports ${ }^{57}$ which have suggested that beyond a certain flow rate, indium 
no longer acts as a surfactant and is instead incorporated into the lattice during ternary alloy formation.

X-Ray Diffraction measurements were taken in order to further characterize the six period DBR structures. Figure 5-9 shows the (0002) reflection of the six period AlN/GaN DBR structure at room temperature. The lattice parameter for the AIN and the GaN can be derived from this type of plot, and although it is not an accurate measurement of the lattice parameter as discussed previously, it can be used to measure the difference between the AlN and GaN $c$-plane lattice parameter which is shown in Figure 5-10. In this figure, the lattice mismatch between the two layers is plotted as a function of the average cracking in each sample. For all samples under investigation in this work, it is clear that the mismatch between the discrete layers is greater than the nominal value denoted by the solid green line. This indicates biaxial strain in one or both of the DBR layers and is most likely due to in-plane tensile strain in the AlN layer resulting in a reduction in the $c$-lattice parameter for that layer. This effectively increases the difference in the measured $c$-lattice parameters between AIN and GaN. For DBR structures designed to reflect at $460 \mathrm{~nm}$ and $485 \mathrm{~nm}$, a similar trend is observed. As the cracking in the samples increases, the

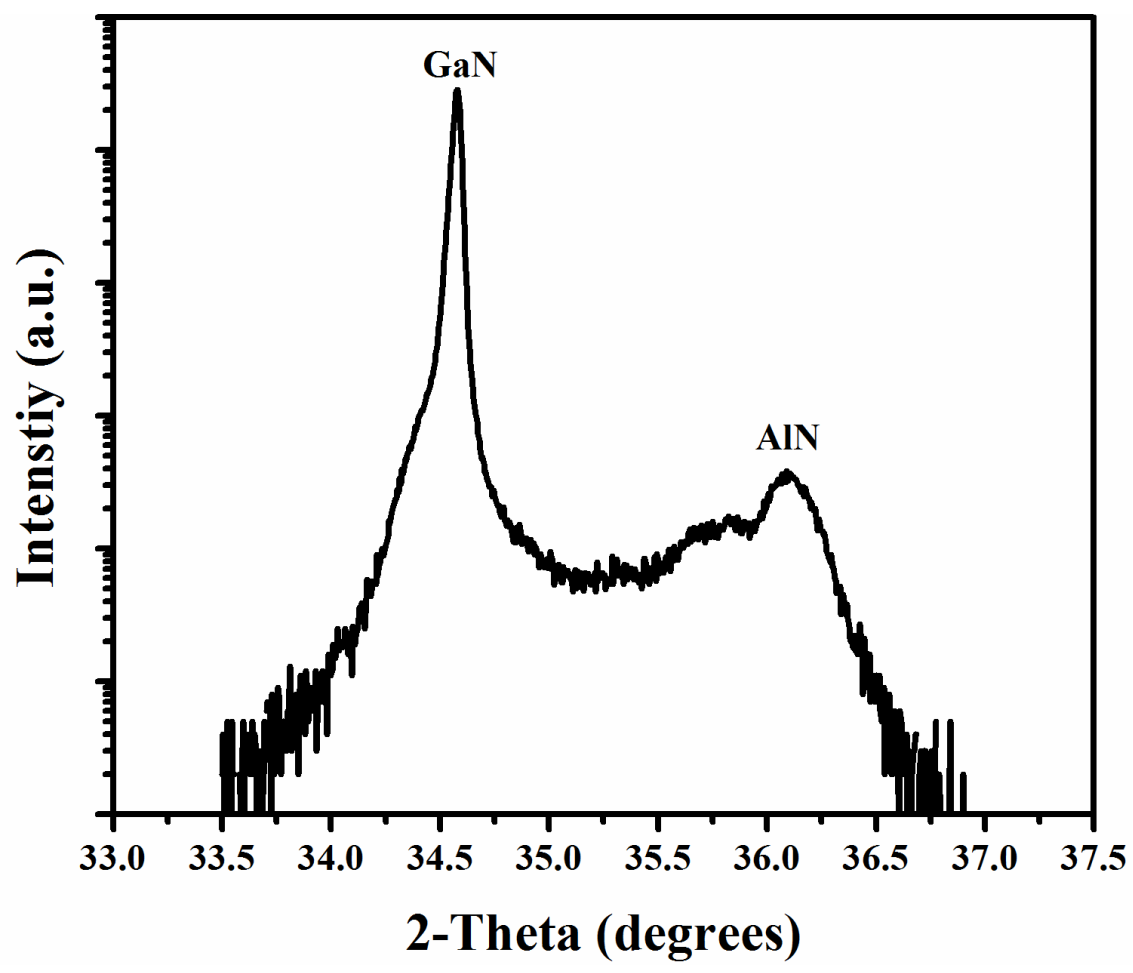

Figure 5-9-(0002) reflection of the six period AlN/GaN DBR. 


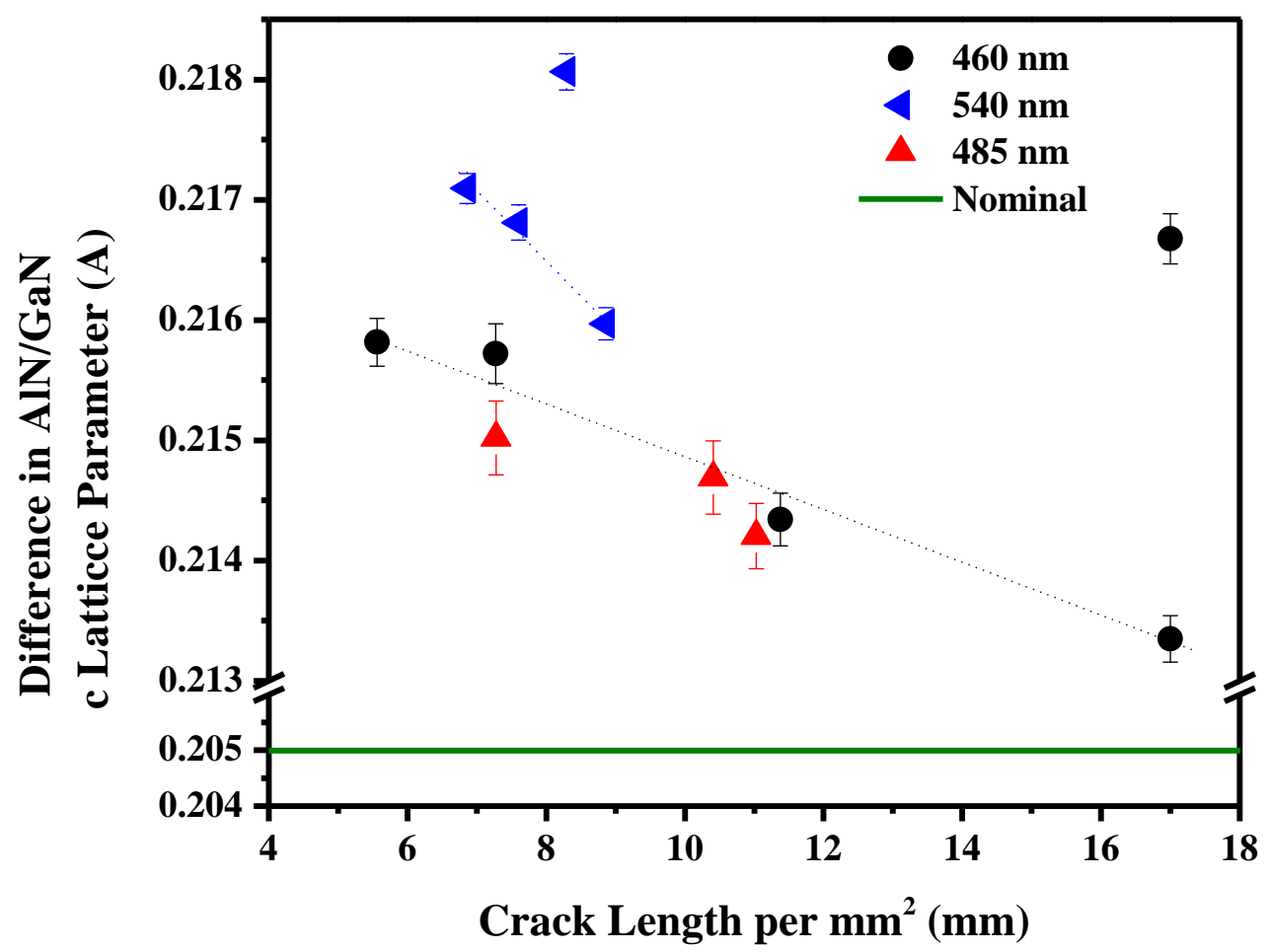

Figure 5-10 - Difference between the AlN and GaN c-lattice parameter derived from the (0002) reflection as a function of the cracking in the films.

difference in the lattice parameters approaches the nominal value. This trend is also evident in the samples reflecting at $540 \mathrm{~nm}$, however, the relaxation appears to have a different slope. Although the mechanism of strain accommodation is not completely understood at this time, it is apparent that the under certain conditions, the indium does allow for the strain between the AlN and $\mathrm{GaN}$ layer to be accommodated with a minimized formation of cracks.

In order to further investigate this effect, the dependence of the lattice parameters and strain was conducted at different temperatures. In particular, a temperature dependent XRD study was conducted. In this technique, the hot stage was affixed to the Bruker D8-Discover system discussed previously. The temperature of the stage was varied from room temperature to $1023 \mathrm{~K}$ and the (0002) scan was evaluated at temperatures in between during both the heating cycle and also during the cooling cycle. Figure 5-11 shows the GaN $c$-lattice parameter in the AlN/GaN DBR grown using indium as a surfactant as a function of temperature. From this figure it is clear that there was no change induced in the lattice parameter by the thermal cycle as the lattice parameter upon cooling down was the same as the initial value measured. The 


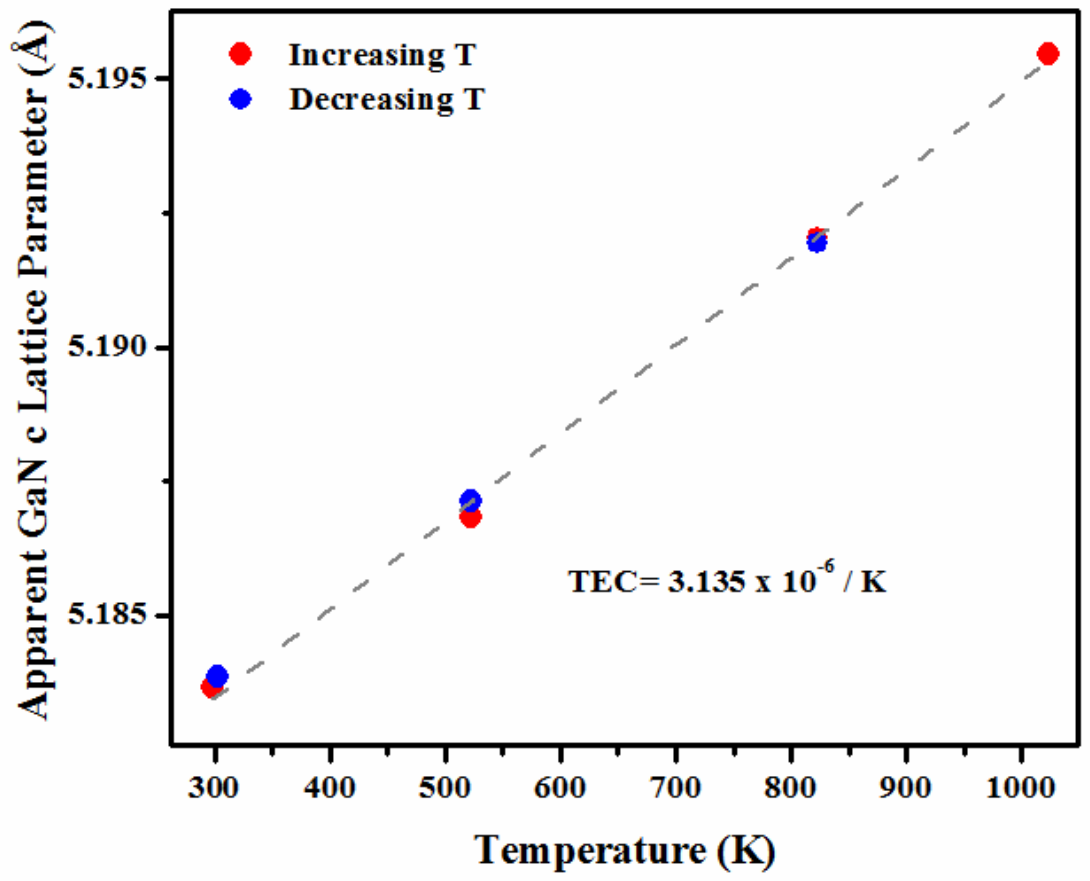

Figure 5-11 - Apparent GaN c-lattice parameter in the AlN/GaN DBR as a funciton of temperature .

extracted GaN thermal expansion coefficient (TEC) is consistent with other values reported in the literature as listed in Table 2-2, although there is some discrepancy between different sources. Figure 5-12 shows the apparent AlN $c$-lattice parameter of the same AlN/GaN DBR as a function of temperature. Unlike that $\mathrm{GaN}$ parameter, the thermal cycle did induce a change in the lattice parameter of the AlN layer. Upon cooling down, the AlN returned to a larger lattice parameter than initially measured. A second (0002) scan was performed on this same sample one day later. The lattice parameter measured then was consistent with that measured after cooling the sample down to room temperature, indicating the change in lattice parameter is not a measurement artifact. As a comparison, the same measurement was performed on AlN/GaN DBR samples grown with no indium. Figure 5-13 and Figure 5-14 show the percent mismatch between the AlN and GaN lattice parameters for DBR samples grown with no indium and also that grown with and TMIn/TMAl ratio of 0.3 . The sample grown with no indium exhibits no change in the lattice parameter mismatch due to the thermal cycle. In this sample, both the AlN and $\mathrm{GaN}$ layers returned to their original lattice parameters. However, the sample grown with a TMIn/TMAl ratio of 0.3 shows a clear change due to the thermal cycle. Upon cooling down, a 


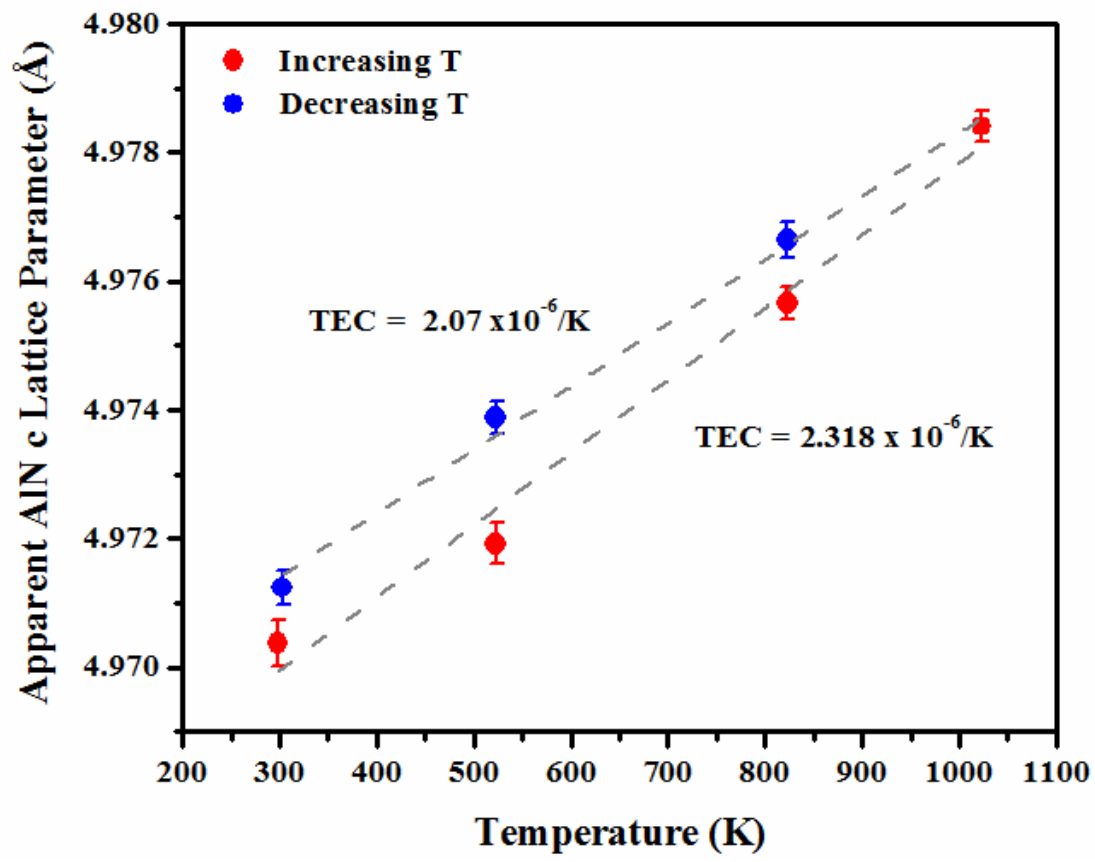

Figure 5-12 - Apparent AlN c-lattice parameter in the AlN/GaN DBR as a funciton of temperature.

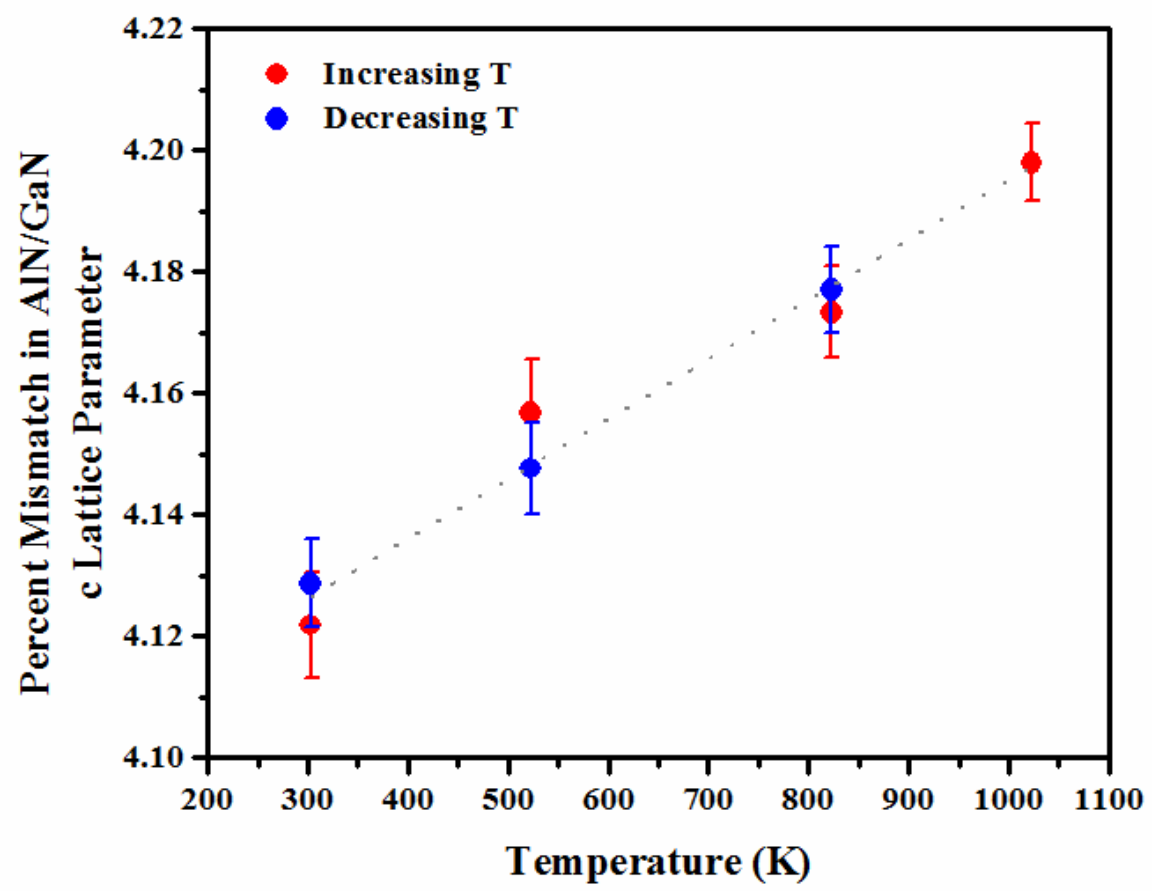

Figure 5-13 - Percent mismatch between the AlN and GaN lattice parameters in DBRs grown with no indium. 


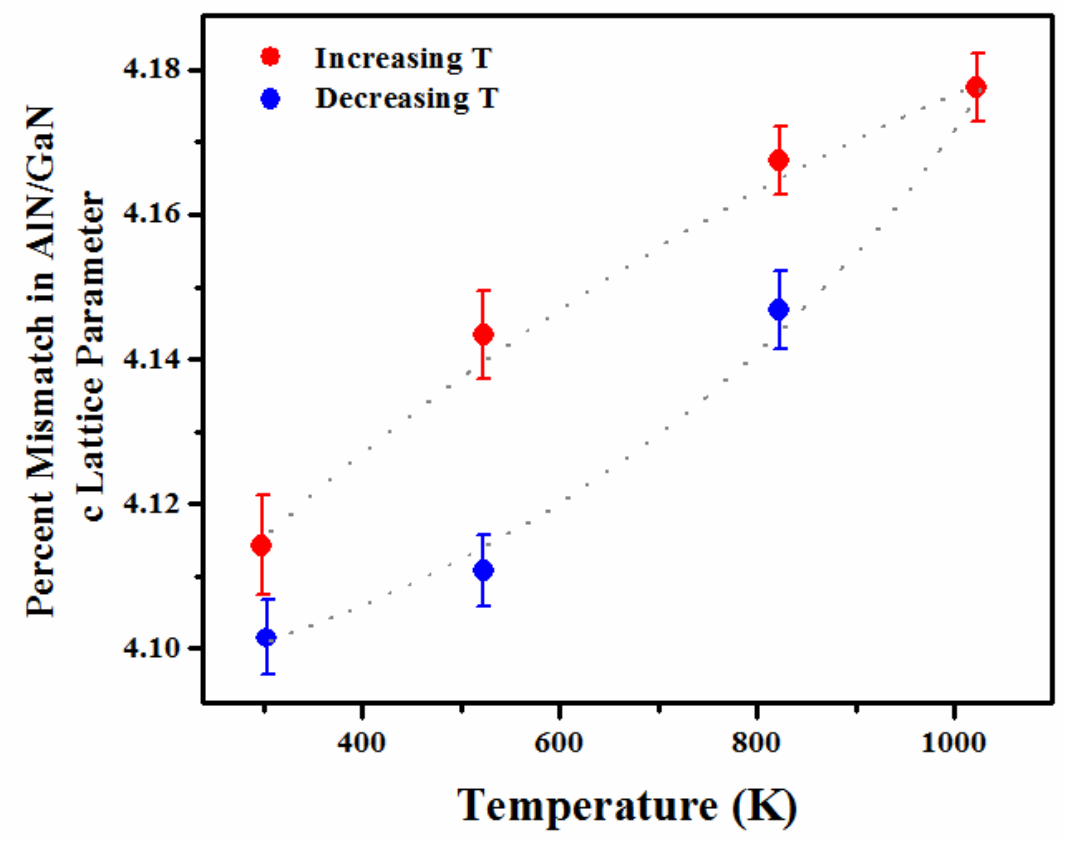

Figure 5-14 - Percent mismatch between the AlN and GaN lattice parameters in DBRs grown with a TMIn/TMAl ratio of 0.3 .

reduction in the mismatch was observed consistent with the increase in the AlN lattice parameter. No additional cracking was observed after the thermal cycle in either sample. From this, it can be concluded that the minimal incorporation of indium may be changing the material properties.

\subsection{DBRs using Strain Relieving Interlayer}

From the discussion above, the use of indium enabled a reduction in the cracking of AlN/GaN DBRs by a factor of two. In order to further accommodate the strain in the AlN/GaN DBRs, a GaN interlayer was inserted in the center of the DBR stack. This was done to effectively decouple the top and bottom stack to minimize the strain accumulation. In order to achieve high reflectivity (>90\%) the targeted structures were 12 and 18 period DBRs. The interlayer was formed by varying the GaN thickness in the sixth or ninth period of the DBR. In addition to changing the strain profile in the superlattice, the interlayer also changes the reflectivity spectra. Through simulations, it was determined that the interlayer must be an odd multiple of a quarter wavelength thickness in order to have the peak reflectivity at the designed wavelength. For even multiples, there is a sharp dip at the target wavelength as shown in Figure

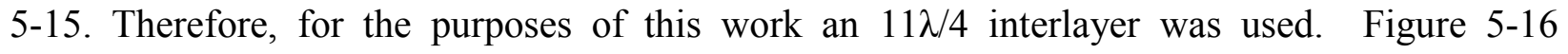


contains the cross sectional SEM image showing the fabricated structure. This technique did yield crack-free DBR structures over a 2" wafer on miscut sapphire substrates, however cracking was observed when grown on non-miscut substrates. Figure 5-17 and Figure 5-18 show the measured and simulated reflectivity spectra for the 12 and 18 period structures. It is clear that the 12 period exhibited a similar shape to the simulated response, however the maximum reflectivity was considerably less. This may be due to the variation in the refractive index of the layers or the thicknesses. The 18 period structure however was closer to the target reflectivity of $90 \%$. In order to be integrated into active devices, the surface morphology of the DBRs needs to be epi-ready. Figure 5-19 shows the surface morphology of $600 \mathrm{~nm}$ of GaN layer grown on the surface of the 12 period DBR showing step morphology and a smooth surface. While this is suitable for device growth, the reflectivity spectrum has unique shape that may not be ideal. Since the amount of cracking observed in this technique was found to be dependent on the sapphire substrate used a more robust technique was pursued.

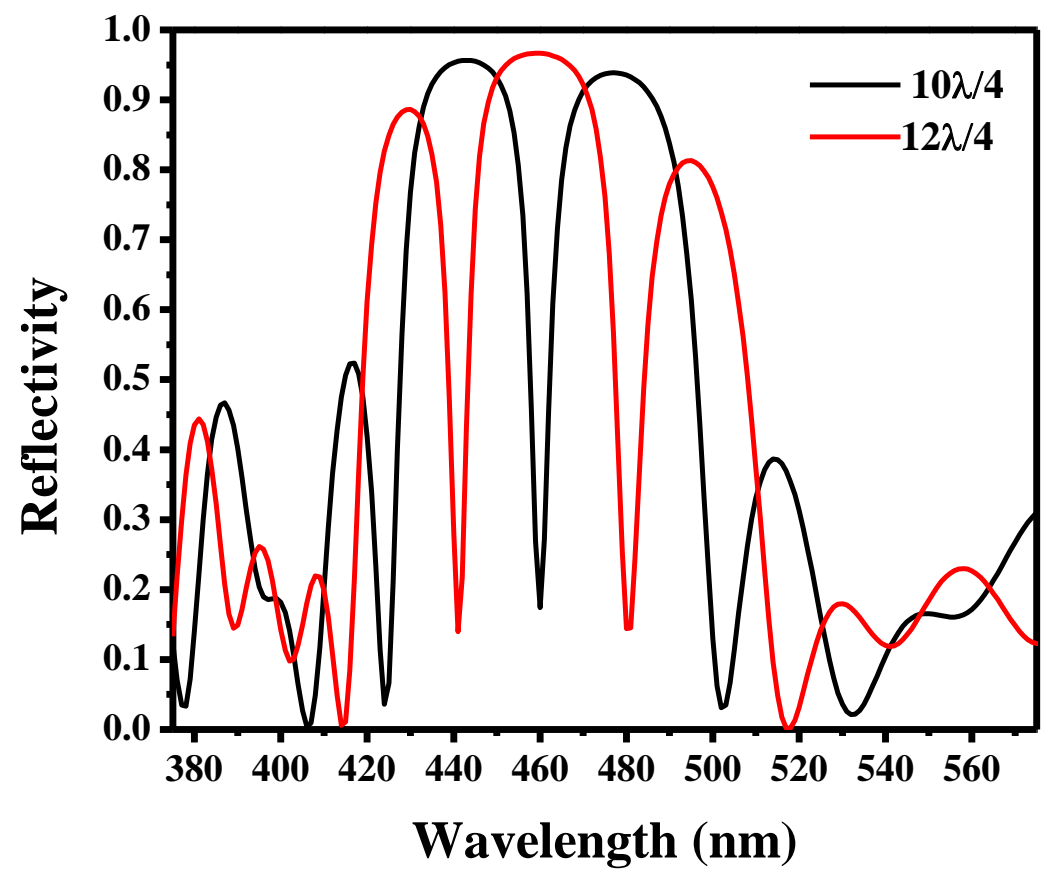

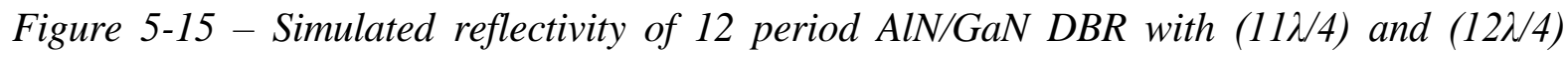
interlayer thicknesses. 


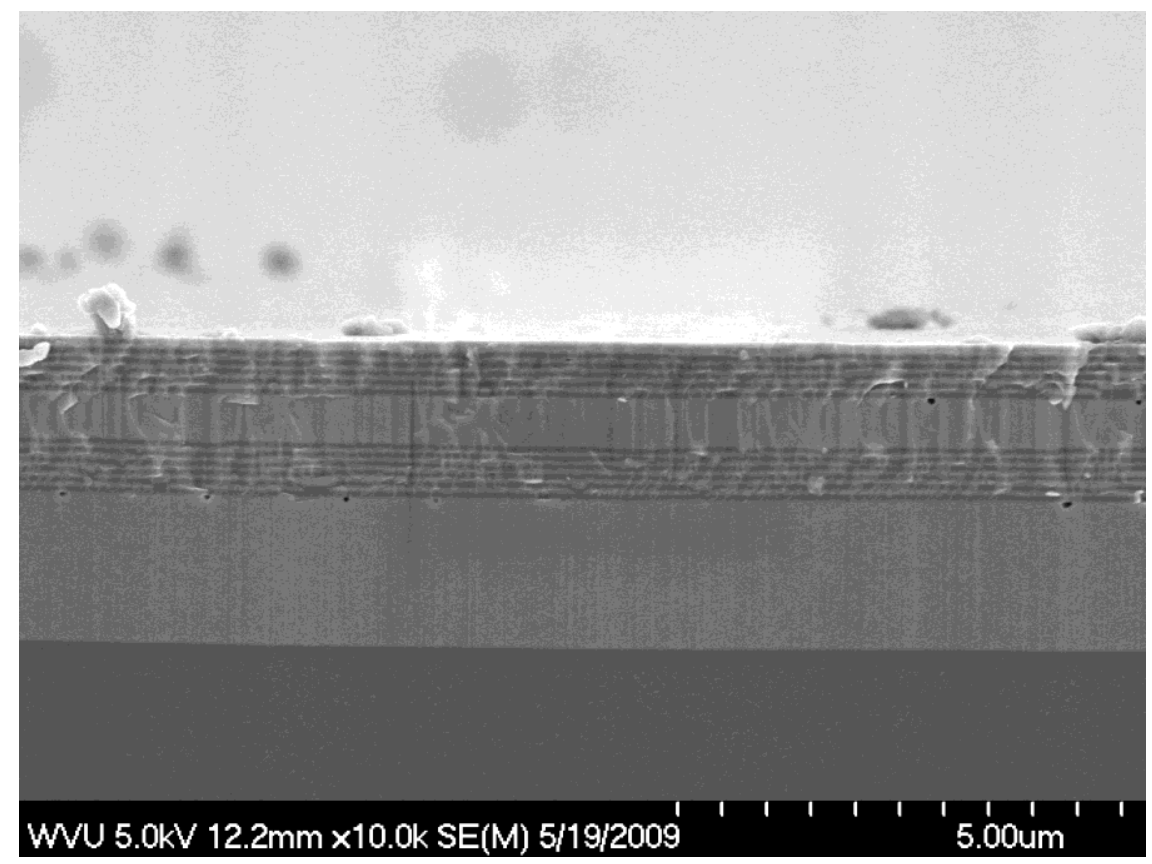

Figure 5-16 - Cross sectional SEM image of 12 period AlN/GaN DBR with 11ג/4 GaN interlayer.

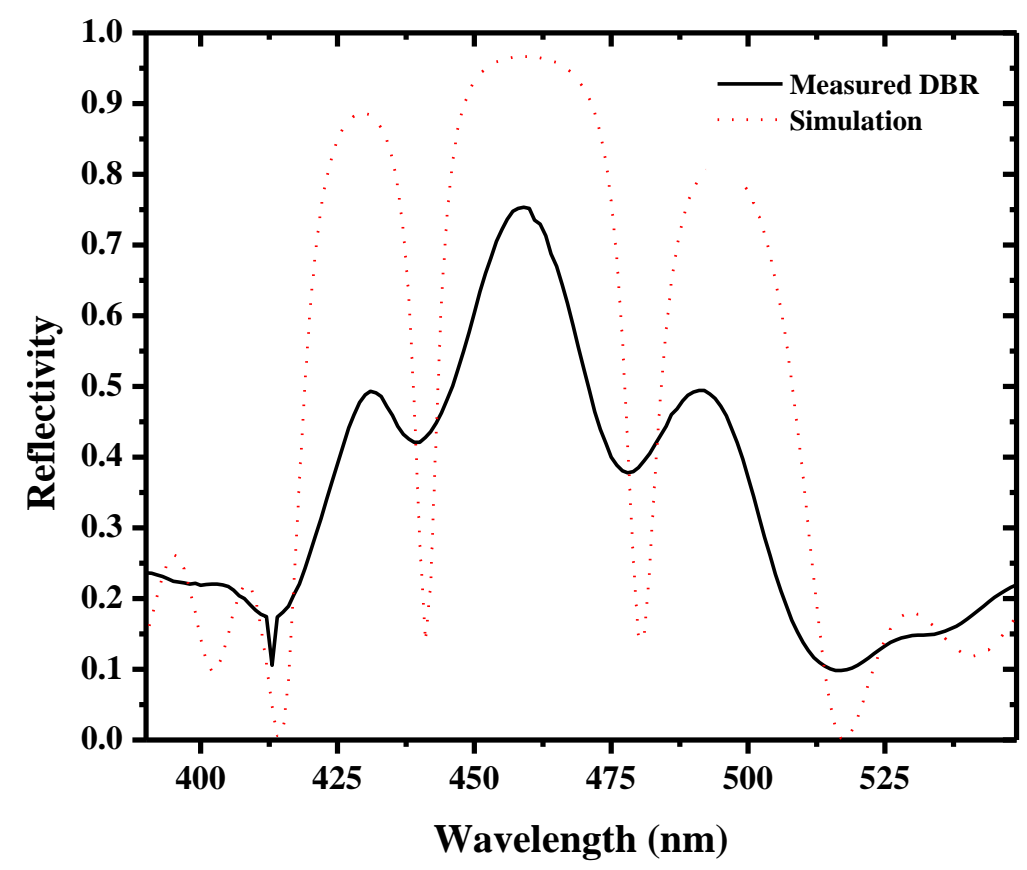

Figure 5-17-Measured reflectivity for 12 period AlN/GaN DBR with 11ג/4 GaN interlayer. 


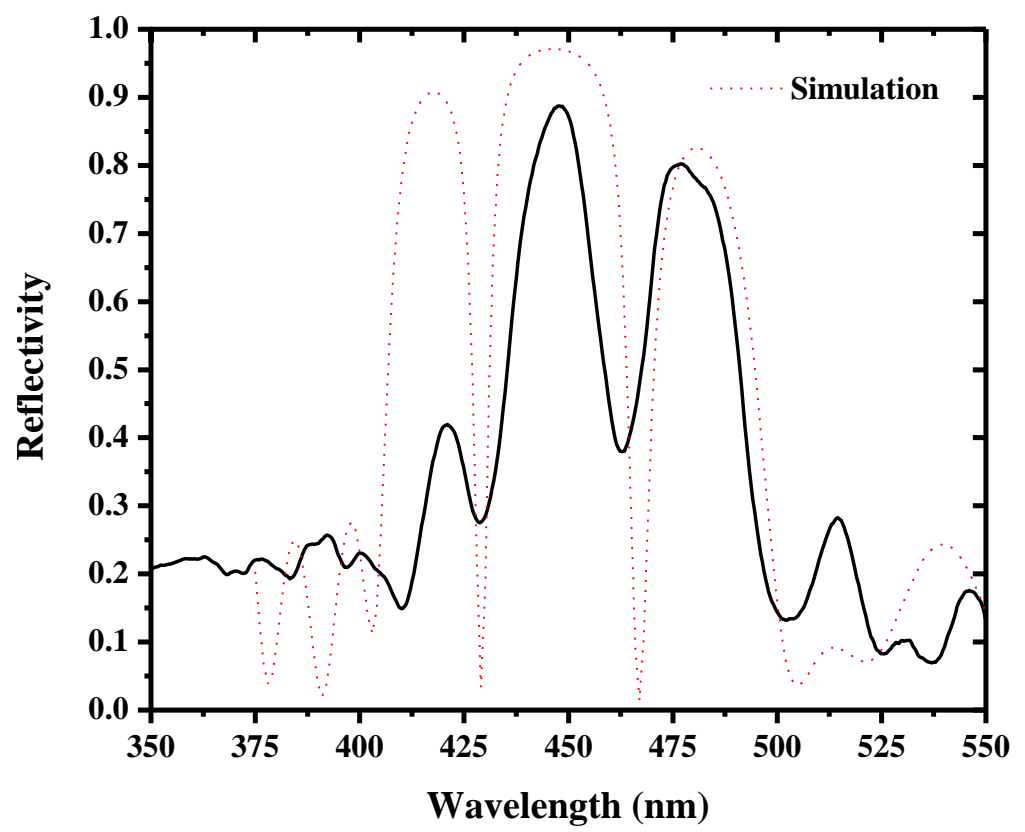

Figure 5-18 - Measuremed reflectivity for an 18 period AlN/GaN DBR with 11N/4 GaN

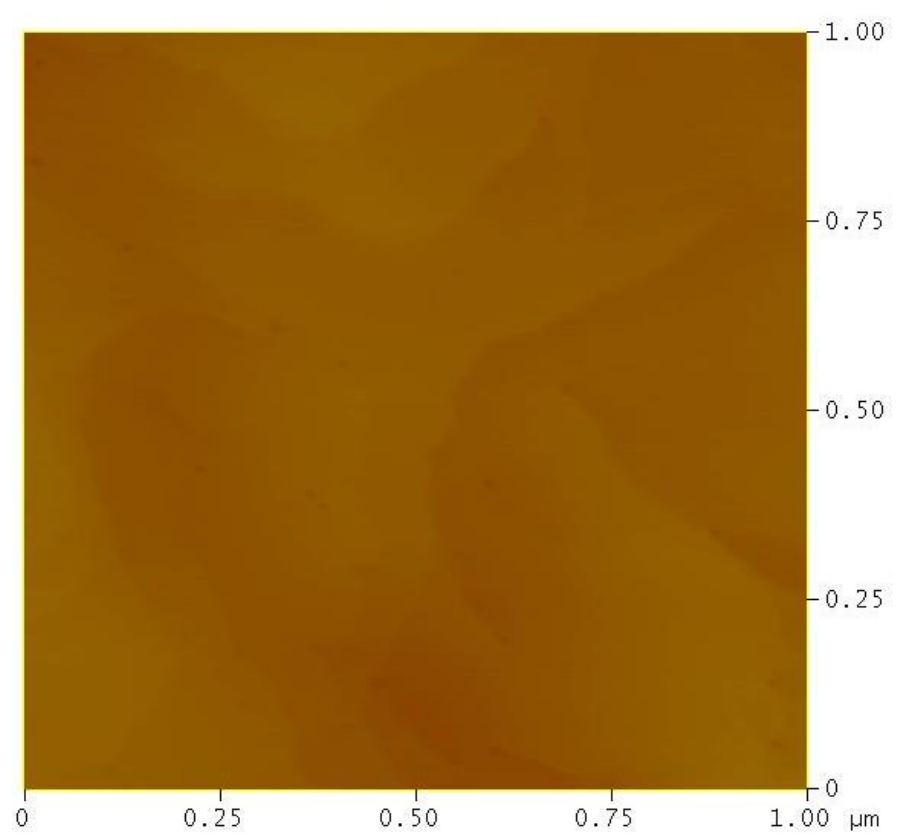

Figure 5-19 - AFM image of $600 \mathrm{~nm} n G a N$ overgrowth on 12 period AlN/GaN DBR with interlayer. Z-scale is $30 \mathrm{~nm}$. 


\subsection{DBR Growth using Digital Alloys}

In this task, the AlN layers in the DBR as shown in Figure 5-3 were replaced with an optimized $\mathrm{Al}_{x} \mathrm{Ga}_{1-x} \mathrm{~N}$ digital alloy having a high $\mathrm{Al}$ content. The targeted $\mathrm{Al}$ concentration was around $90 \%$ in order to maximize the refractive index contrast between the layers to minimize the number of periods required. As described previously, superlattice structures have been inserted in III-Nitride based DBRs ${ }^{76,78}$ and used to reduce cracking. In this technique a superlattice in integrated into each period of the DBR, as shown in Figure 5-20, to relieve strain. The growth conditions for the digital alloy are the same as those discussed in Chapter 4. Structures with periods ranging from 6 to 25 have been fabricated in order to achieve high reflectivity DBRs which can be used in LED device fabrication. These structures have been evaluated via SEM, XRD, and AFM to verify the structural integrity.

All the DBR structures were grown in the Aixtron MOVPE system at WVU. The structures were grown on $c$-plane sapphire substrates with a $\sim 2 \mu \mathrm{m} \mathrm{GaN} \mathrm{buffer} \mathrm{layer.} \mathrm{The}$ growth conditions discussed previously for the $90 \% \mathrm{Al}$ content digital alloy was used for the $\mathrm{Al}_{x} \mathrm{Ga}_{1-x} \mathrm{~N}$ layer. This corresponds to a $1 \mathrm{~s} \mathrm{GaN}$ growth time and a $188 \mathrm{~s}$ AlN growth time. Two different growth conditions were investigated for the GaN layer. In the first method, the GaN layer was grown at the conditions optimized for $\mathrm{GaN}$ on sapphire growth.

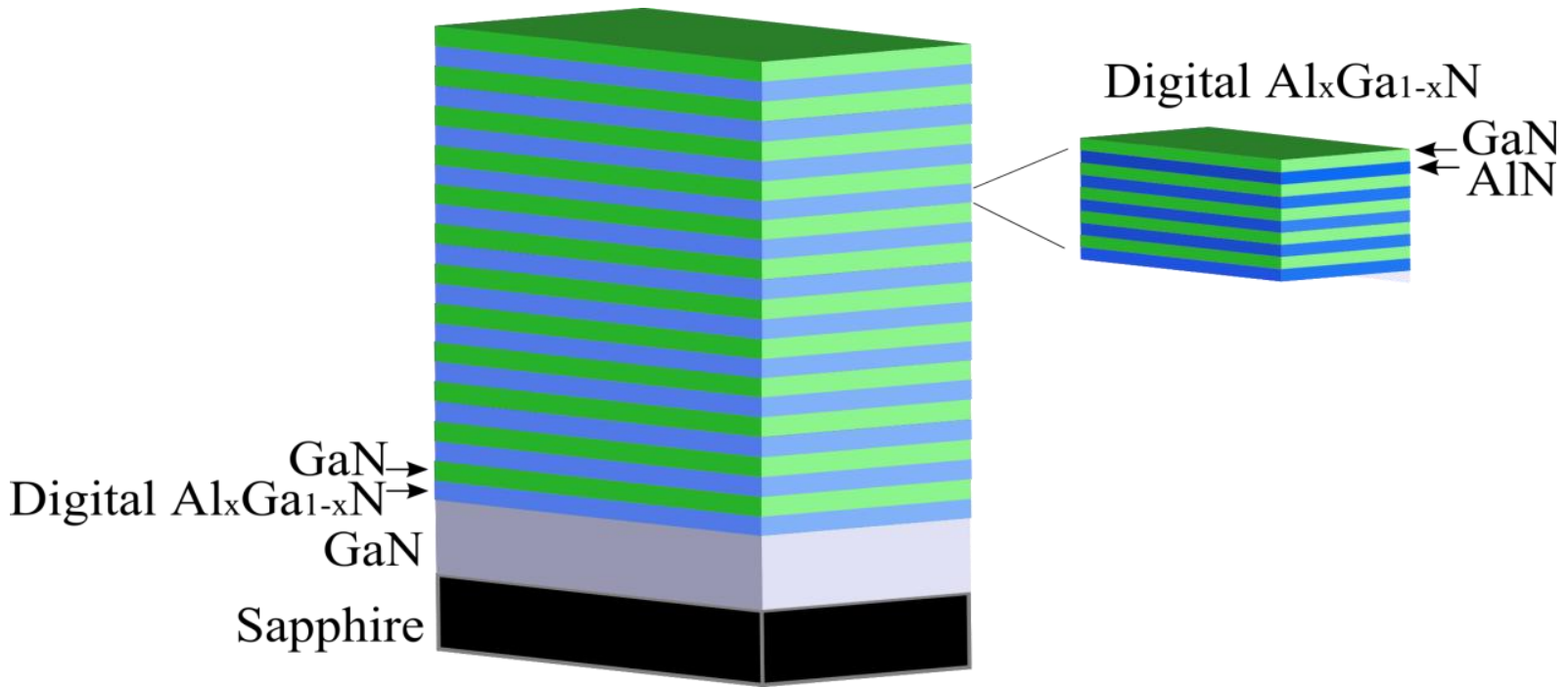

Figure 5-20 - Schematic of DBR containing GaN and digital $A l_{x} G a_{1-x} N$ layers. 
These are the same conditions used for the underlying buffer layer. Figure 5-21 shows the cross sectional SEM image of a 12 period DBR grown under these conditions. The interfaces appear sharp, however, the GaN layer thickness does not appear uniform throughout the periods. Therefore, the growth conditions for the GaN layer were modified in order to minimize switching times between the layers. The growth conditions used for the $\mathrm{GaN}$ in the digital alloy were the employed for the GaN layer in the DBR. Figure 5-22 contains a cross sectional SEM image of a 25 period DBR grown under the modified conditions. In these structures, the GaN layer was considerably more uniform throughout each layer. Therefore, these growth conditions were used in subsequent characterization and device fabrication.

DBR structures with varying number of periods have been fabrication and the peak reflectivity followed the calculated reflectivity as is shown in Figure 5-23. The following equation $^{81}$ was used to calculate the relationship between the reflectivity and the number of periods assuming $n_{\mathrm{GaN}}=2.4, n_{\mathrm{AlGaN}}=2.17$, and $n_{\mathrm{Sap}}=1.78$ :

$$
R_{t h}=1-4\left(\frac{n_{L}}{n_{H}}\right)^{2 N_{\text {pair }}} \frac{n_{\text {sub }}}{n_{H^{2}}}
$$

In this expression, $n_{L}$ is the refractive index of the lower index material, $n_{H}$ is the refractive index of the higher index material, and $n_{\text {sub }}$ is the refractive index of the substrate. The experimentally measured reflectivity for several representative DBR samples with differing number of periods is shown in Figure 5-24. For these samples, the targeted reflectivity was around $460 \mathrm{~nm}$. It can be seen from this figure that that DBRs with up to 25 periods have been fabricated, which a maximum reflectivity around $95 \%$. In order to fully evaluate the 25 period digital $\mathrm{Al}_{x} \mathrm{Ga}_{1}$ ${ }_{x} \mathrm{~N} / \mathrm{GaN}$ DBR, a corresponding 25 period AlN/GaN DBR was also fabricated. Figure 5-25 shows the surfaces of the two DBR samples. The digital $\mathrm{Al}_{x} \mathrm{Ga}_{1-x} \mathrm{~N} / \mathrm{GaN}$ DBR was characterized by a crack-free surface, while the corresponding AIN/GaN DBR contained the network of cracks typical in such growths. It should also be noted that samples containing digital alloys with fewer than 25 periods were also characterized by a crack-free surface. This verifies that the digital alloy can be used for strain relief and crack suppression in high reflectivity structures. Figure 5-26 shows the reflectivity for the two structures. While the peak reflectivity is the similar, the stop band width of the digital $\mathrm{Al}_{x} \mathrm{Ga}_{1-x} \mathrm{~N} / \mathrm{GaN}$ DBR is slightly less than the corresponding $\mathrm{AlN} / \mathrm{GaN} \mathrm{DBR}$ due to the reduced refractive index contrast. 

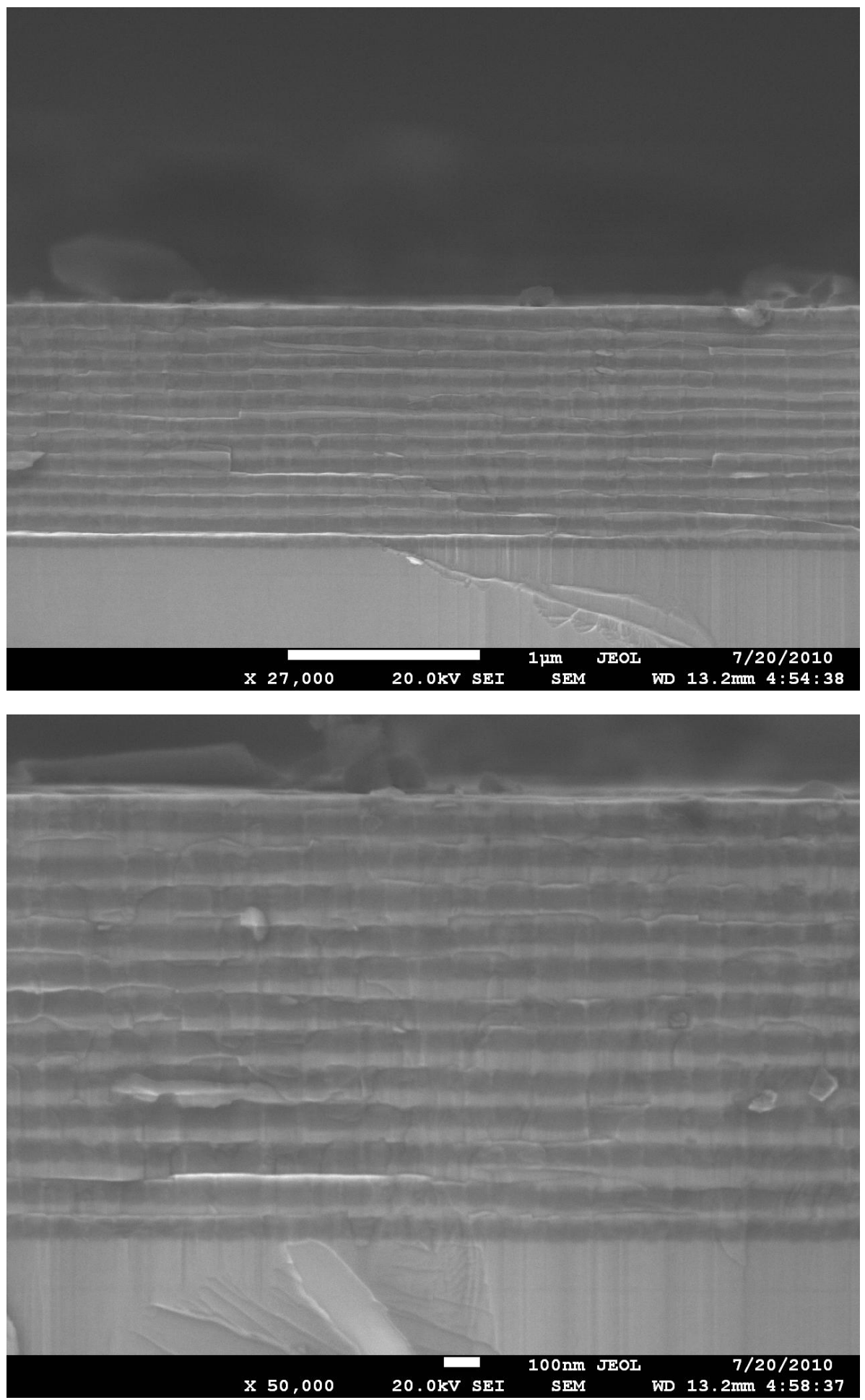

Figure 5-21 - Cross sectional SEM image of a 12 period digital $A l_{\mathrm{x}} G a_{1-\mathrm{x}} N / G a N D B R$. 

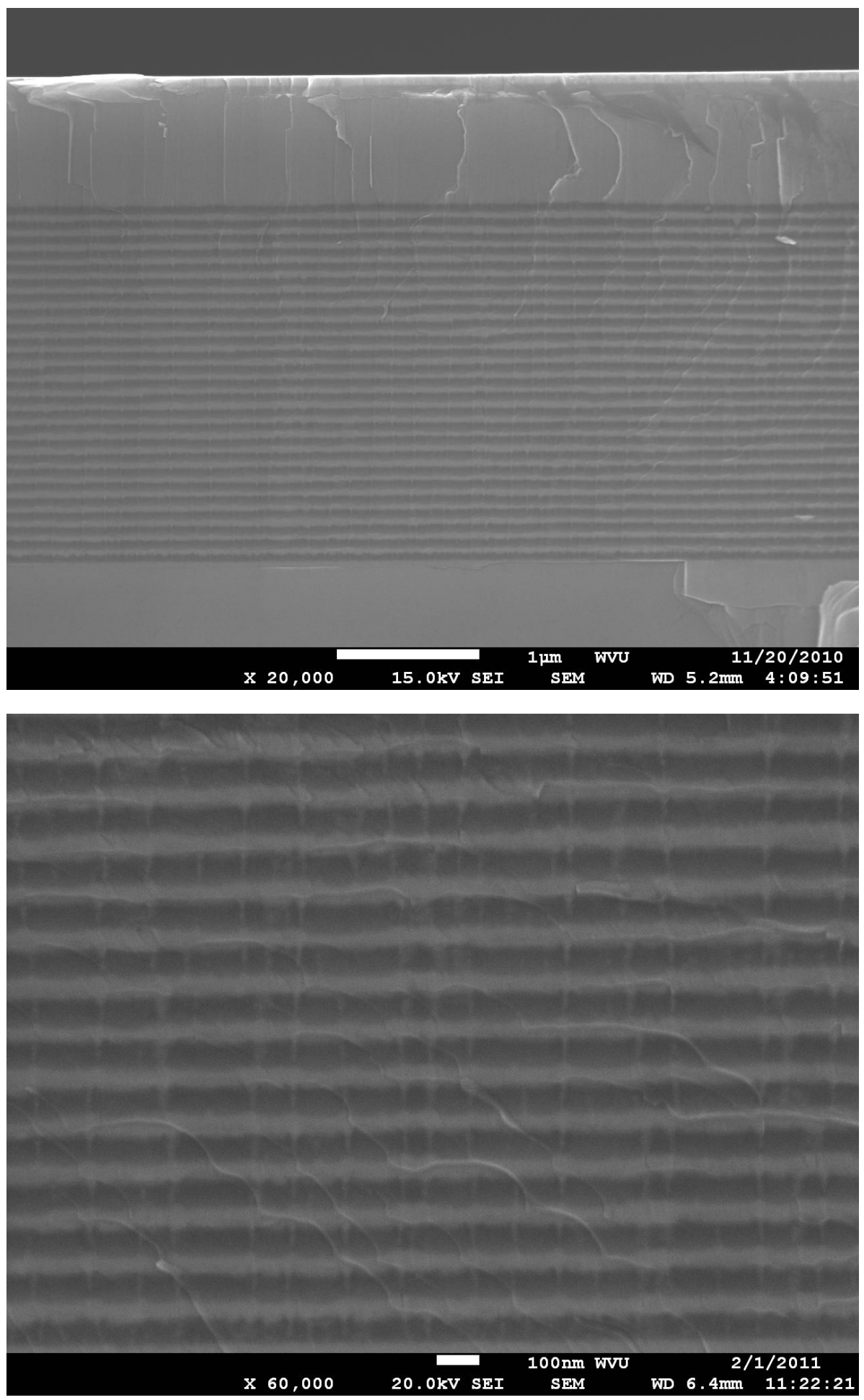

Figure 5-22 - Cross sectional SEM image of 25 period digital $A l_{\mathrm{x}} G a_{1-\mathrm{x}} N / G a N D B R$ in which the growth conditions have been modified for faster switching times. 


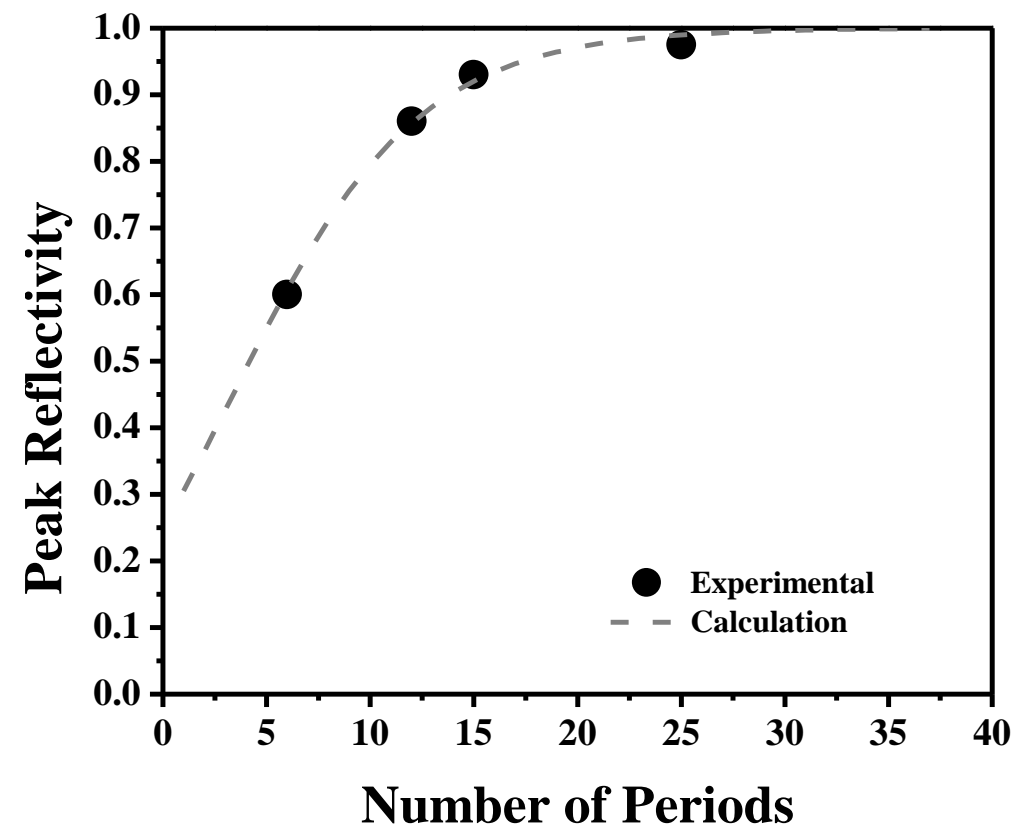

Figure 5-23 - Reflecitivty of Digital $A l_{\mathrm{x}} G a_{1-\mathrm{x}} N / G a N D B R$ as a function of the number of periods.

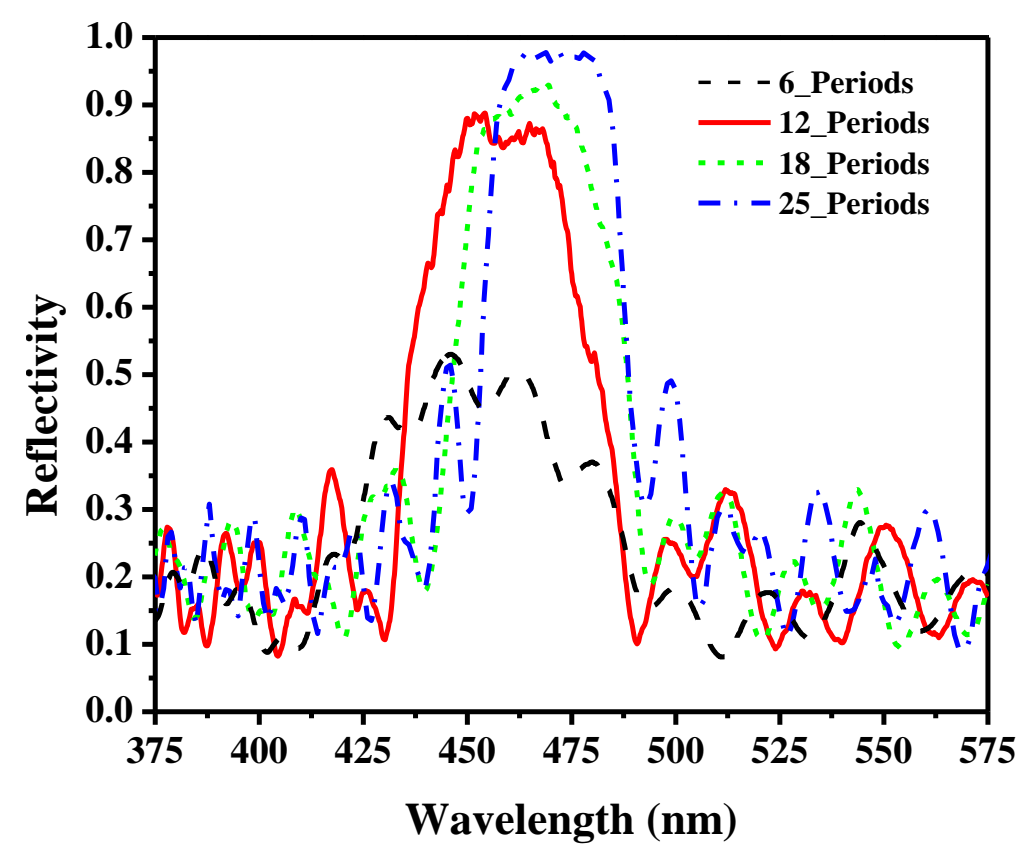

Figure 5-24 - Reflectivity of digital $A l_{\mathrm{x}} G a_{1-\mathrm{x}} N / G a N D B R s$ with total periods ranging from 6 to 25 . 


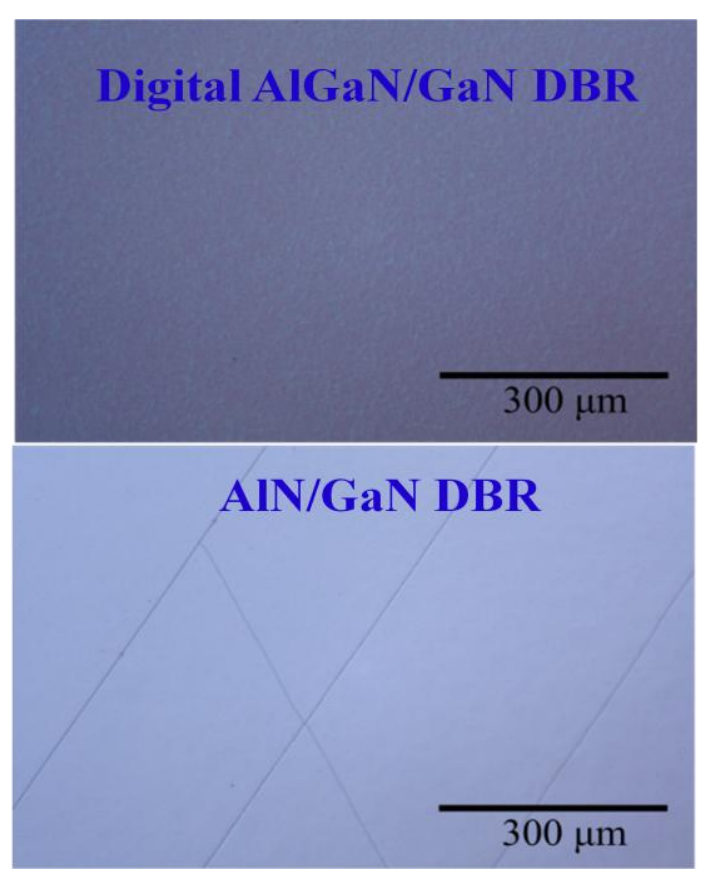

Figure 5-25 - Microscope images of the surface of a 25 period digital $A l_{\mathrm{x}} G a_{1-\mathrm{x}} N / G a N D B R$ and a 25 period AlN/GaN DBR.

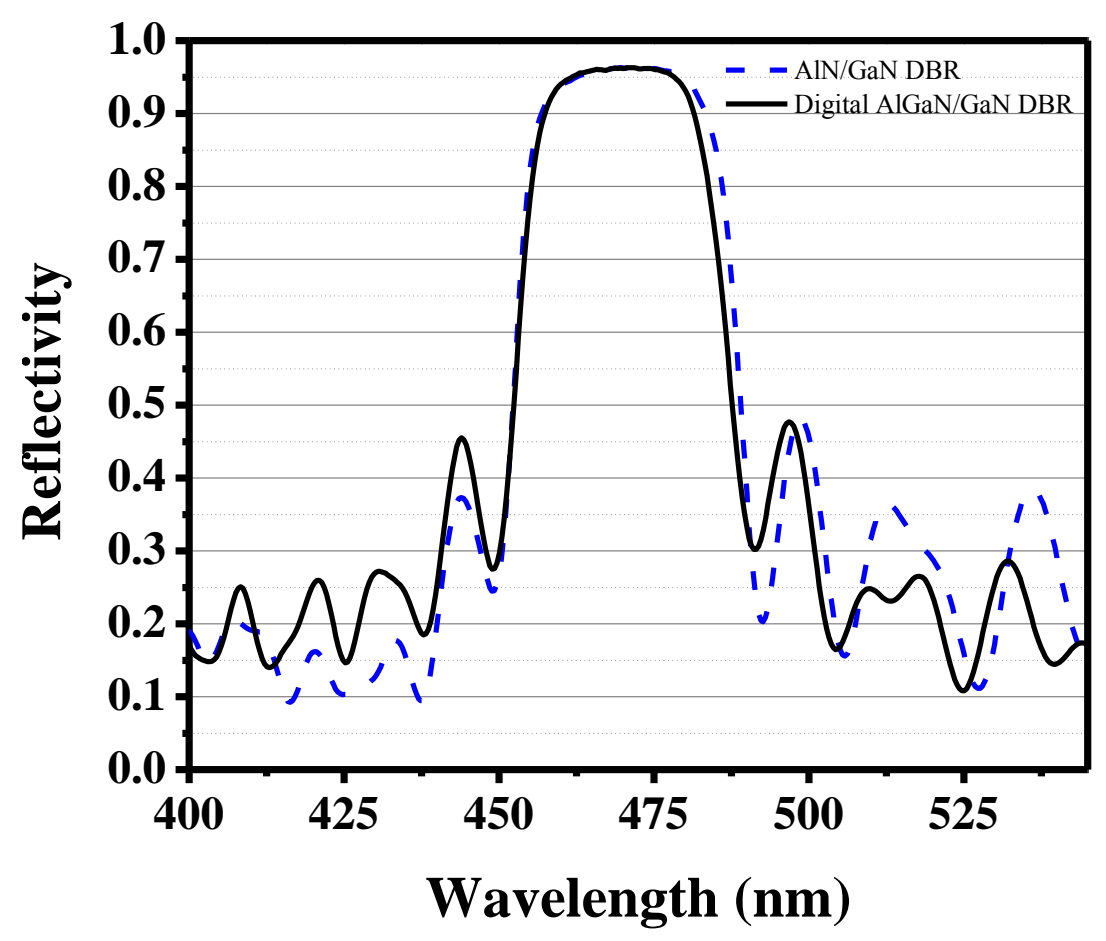

Figure 5-26 - Reflectivity of 25 period digital $A l_{x} G a_{1-x} N / G a N$ DBR and 25 period AlN/GaN DBR. 
The digital DBRs were further characterized by XRD in order to evaluate the structure. Figure 5-27and Figure 5-28 contain an on-axis scan and an off-axis scan respectively. From the on-axis scan of the (0002) peak, it is shown that the $\mathrm{Al}_{x} \mathrm{Ga}_{1-x} \mathrm{~N}$ in the DBR corresponds to the targeted $90 \% \mathrm{Al}$ concentration, which is determined by the lattice parameter extracted from the peak position. This is in contrast to the AlN peaks in the AlN/GaN DBR which is also shown in Figure 5-27. The off-axis scan maps the (1014) reflection of the digital DBR. In this scan, the $\mathrm{GaN}$ and $\mathrm{Al}_{x} \mathrm{Ga}_{1-x} \mathrm{~N}$ peak are not coherent which is indicated by the vertical mis-alignment of the two peaks. Although the digital DBRs are crack free, it is not a coherent growth. The relaxation may be occurring through some mechanism other than cracking, perhaps defect generation or surface roughening.

In this work, the target was 2" crack free DBRs with consistent reflectivity over the 2" wafer. While samples sizes around $1 / 4$ of a $2 "$ wafer exhibited crack-free surfaces, due to nonuniformities in the growth rate across the 2" wafer, cracking was observed at

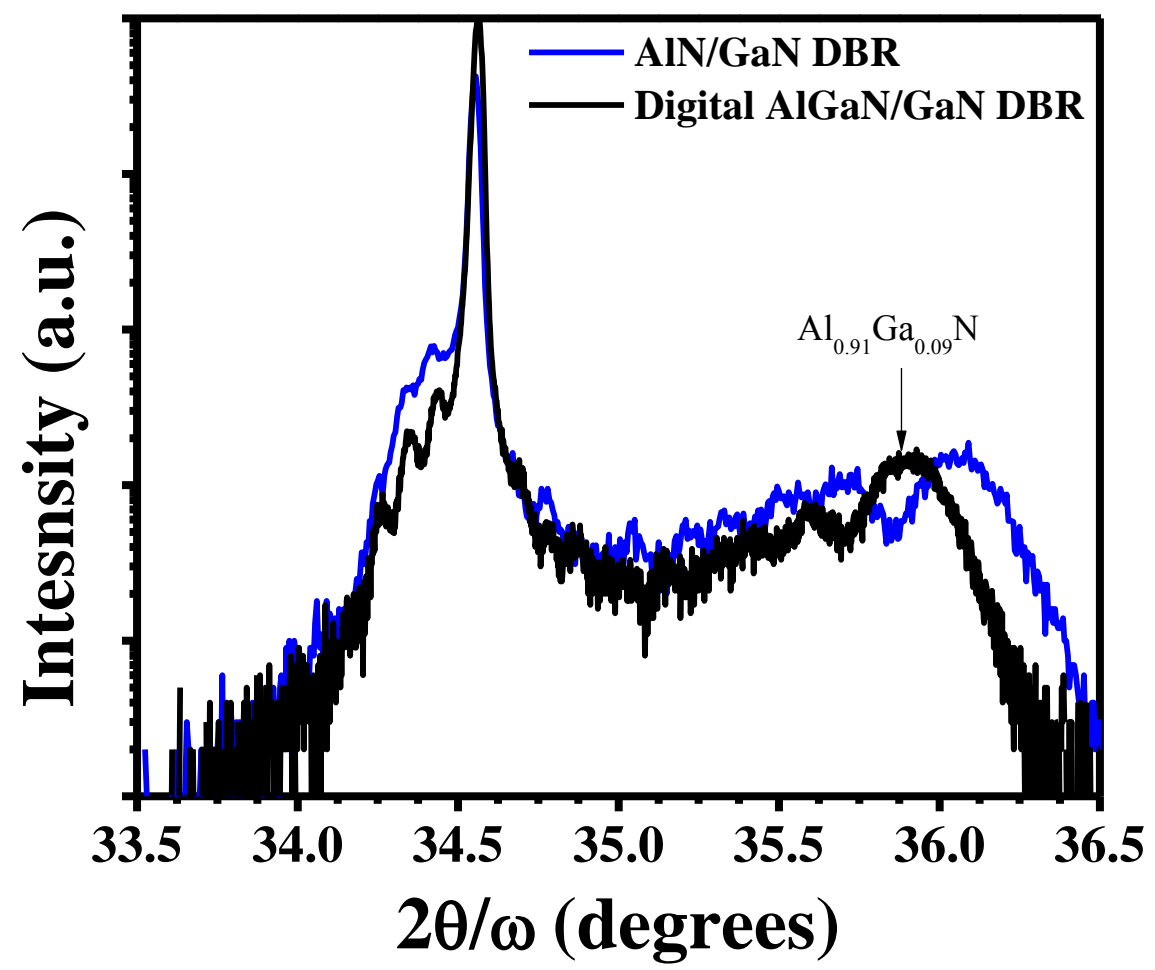

Figure 5-27 - (0002) reflection of 25 period digital $A l_{x} G a_{1-x} N / G a N D B R$ and 25 period AlN/GaN DBR. 
the edge of 2" DBR growths. In this region the peak reflectivity was also red shifted indicating thicker layers at the edge of the wafer. Figure 5-29 shows the cracking as a function of the radial distance from the center of the wafer. This measurement was performed using images taken over the surface of the wafer. In general, cracking was present 3-6 $\mathrm{mm}$ from the edge of the wafer. Figure 5-30 shows the value of the reflectivity over a 2" wafer for a DBR designed to reflect at $452 \mathrm{~nm}$. Close to the edge of the wafer, there is an observable decrease in the reflectivity value as the peak reflectivity shifts away from the targeted value of $452 \mathrm{~nm}$. As mentioned previously, it was experimentally observed that the peak reflectivity shifts to longer wavelengths toward the outer edge of the wafer as shown in Figure 5-31.

One of the last metrics for this growth technique that was evaluated was the transferability. In particular, it was the goal to develop a growth technique that could be transferred easily between different substrates with few modifications required. To verify the versatility of this digital DBR growth technique, a 20 period digital $\mathrm{Al}_{x} \mathrm{Ga}_{1-x} \mathrm{~N} / \mathrm{GaN}$ DBR was grown on several different substrates. The cracking, surface roughness, and reflectivity were evaluated in order to determine any differences induced by the substrates. In particular, two different types of non-miscut sapphire and a $\mathrm{GaN}$ epilayer on a patterned sapphire substrate (PSS) provided by a collaborator were investigated. The sapphire was from different vendors and had different specifications. 20 period digital $\mathrm{Al}_{x} \mathrm{Ga}_{1-x} \mathrm{~N} / \mathrm{GaN}$ DBRs were grown on these substrates with no modifications made to the recipes. All three substrates exhibited minimal cracking around the edge of the wafer only. Figure 5-32 contains the AFM images of the surface morphology both before and after the DBR growth. For the two samples grown using the nonmiscut sapphire, the RMS roughness after growth was less than $1 \mathrm{~nm}$. There was a considerable increase in surface roughness for the DBR on the PSS indicating that minor modification may need to be made in the growth to correct for this. However, since the reflectivity and cracking were similar as shown in Figure 5-33, this technique appears to be fairly transferable. 


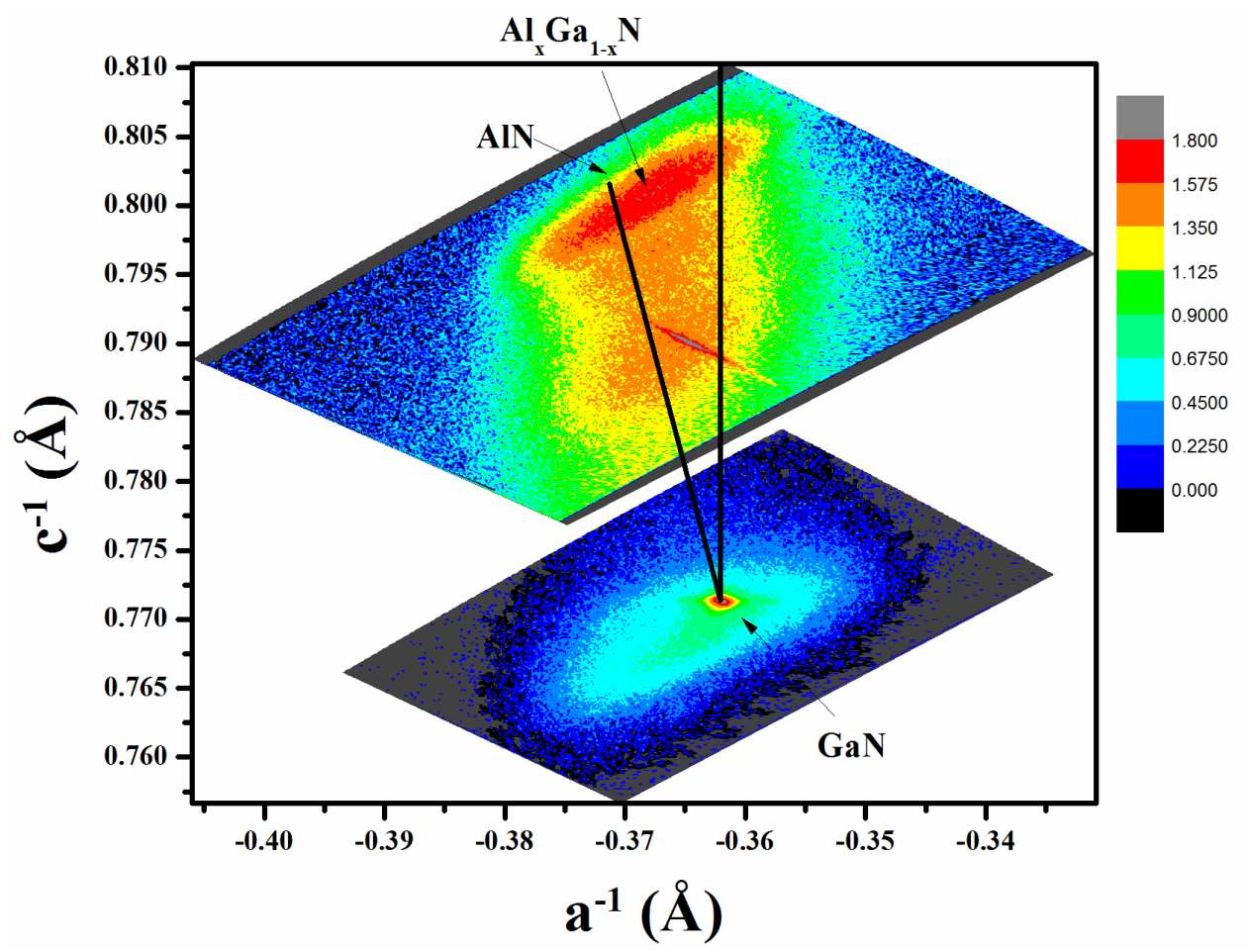

Figure 5-28 - Reciprocal space map of (-104)reflection for 25 period digital $A l_{x} G a_{1-x} N / G a N$ DBR.

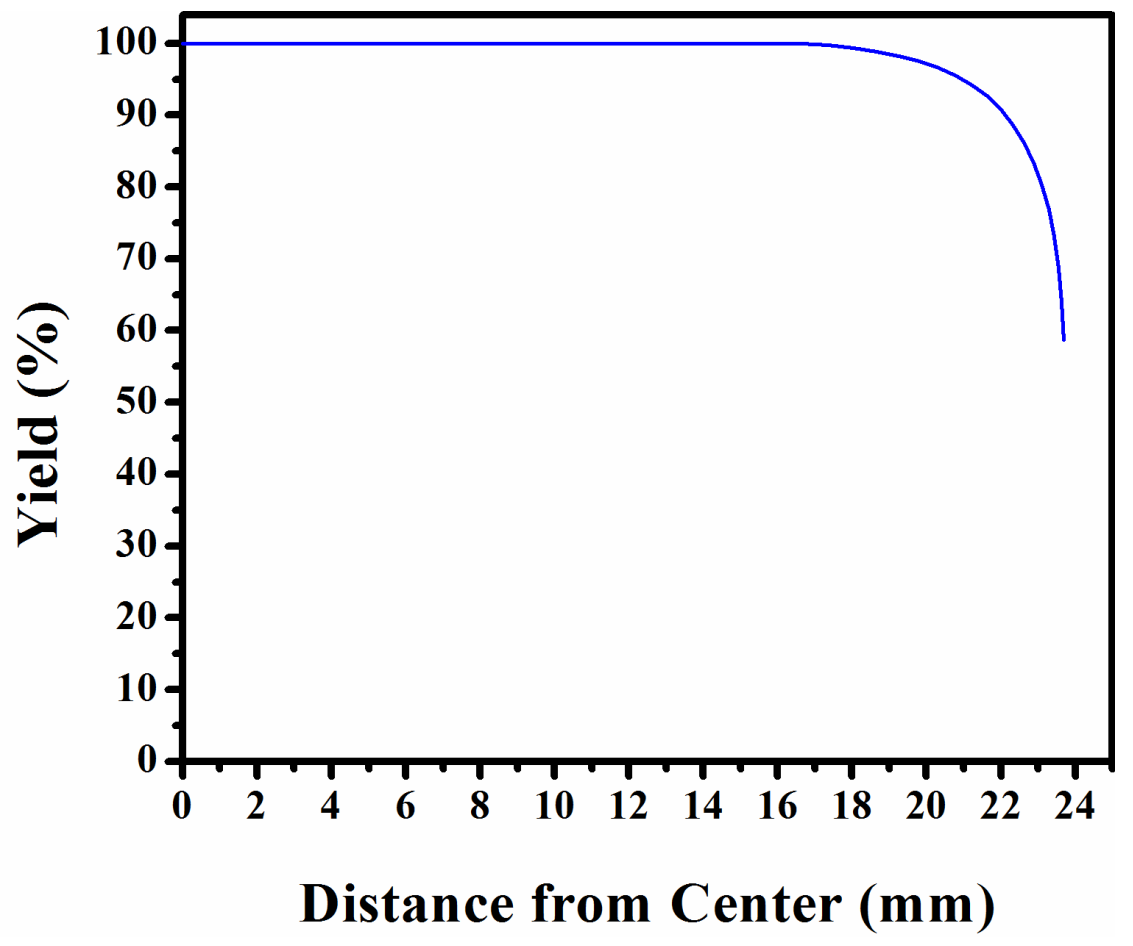

Figure 5-29-Cracking in the 2" DBR from the center of the wafer to the edge. 


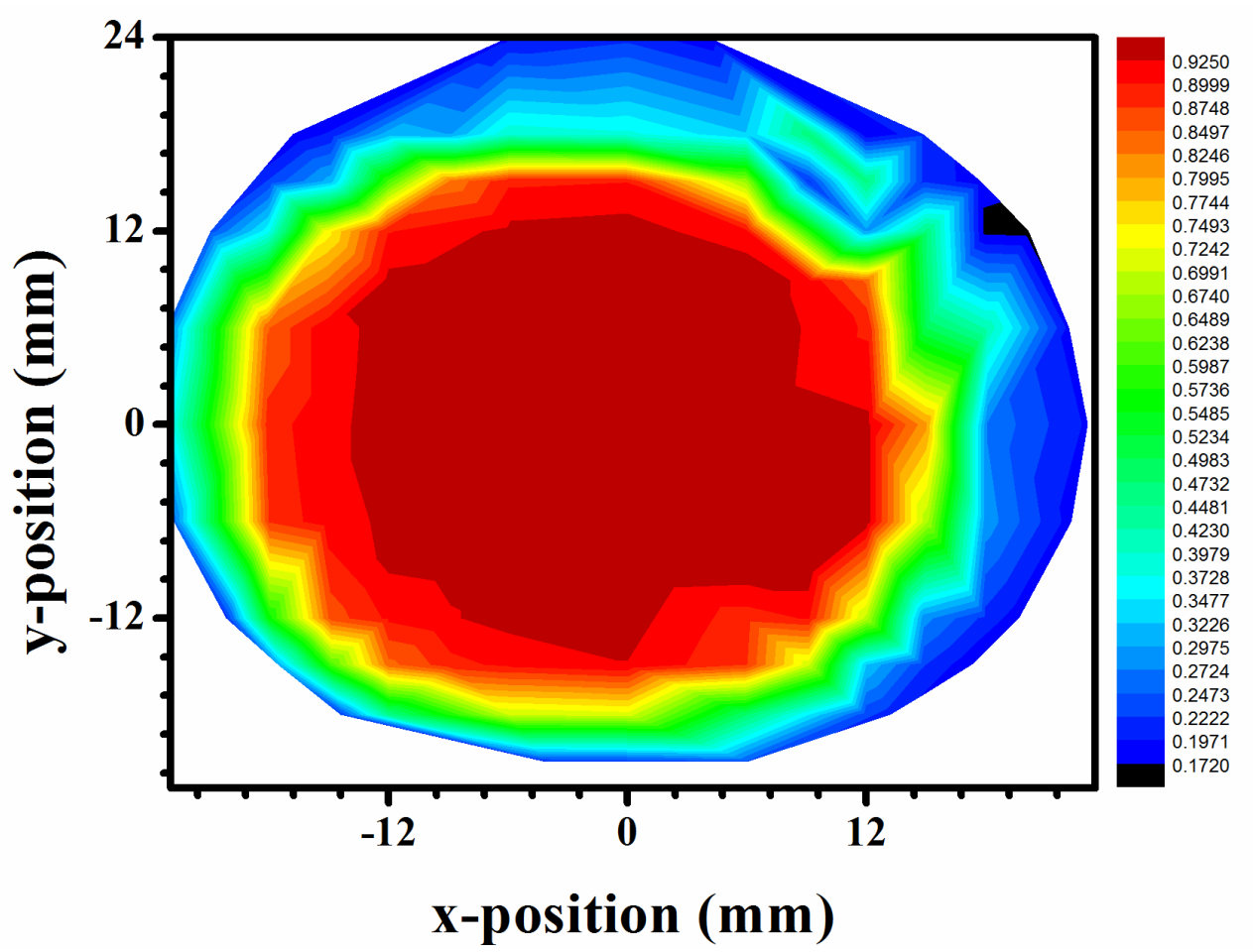

Figure 5-30 - Reflectivity value at $452 \mathrm{~nm}$ mapped over a 2” DBR.

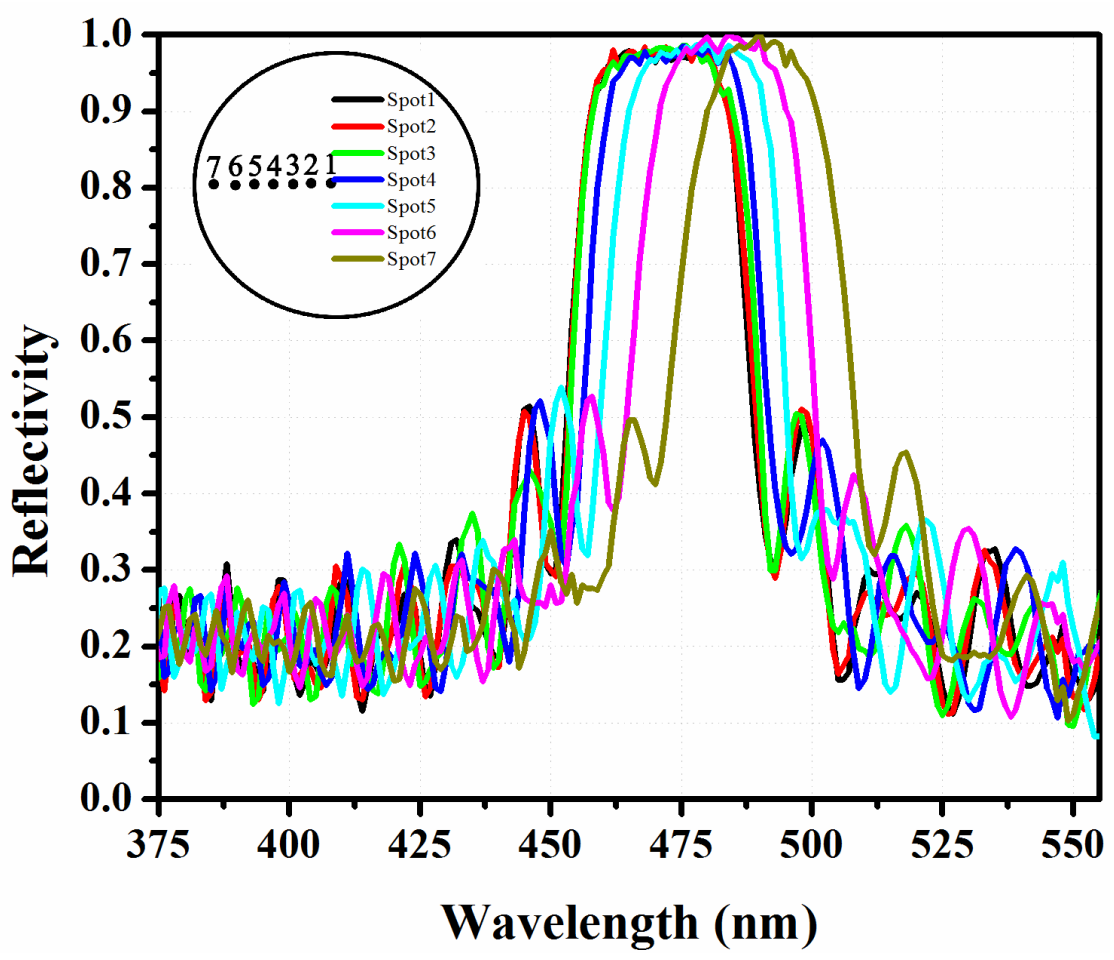

Figure 5-31 - Reflectivy of 25 period DBR at different points along the 2" wafer. 


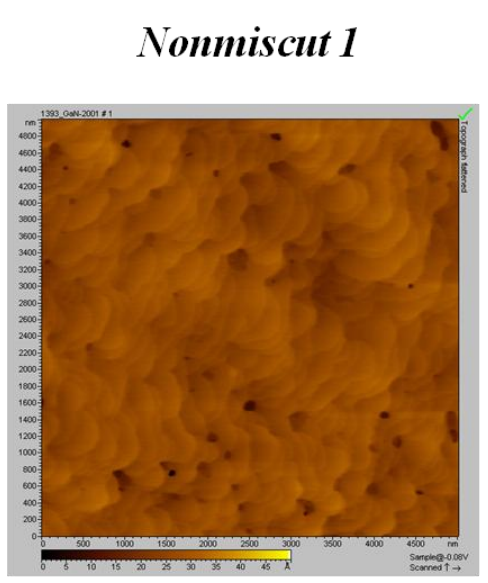

RMS: 0.82 nm

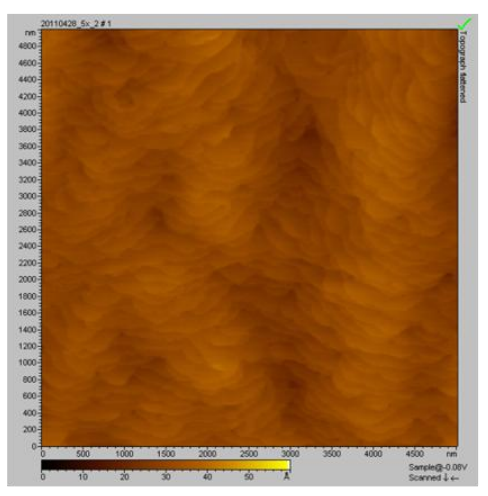

RMS: $0.92 \mathrm{~nm}$

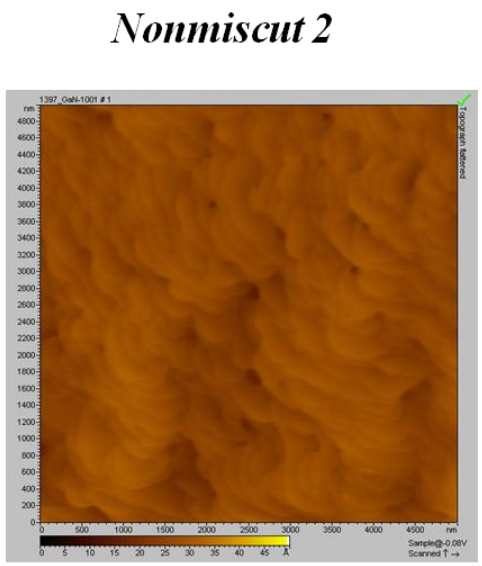

RMS: 0.72 nm

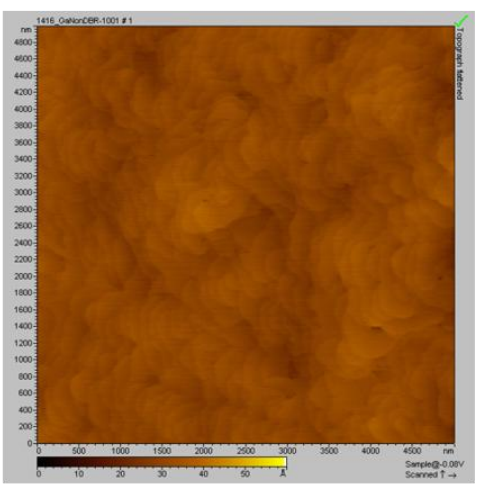

RMS: $0.68 \mathrm{~nm}$
LED Industry ProducedGaN on PSS

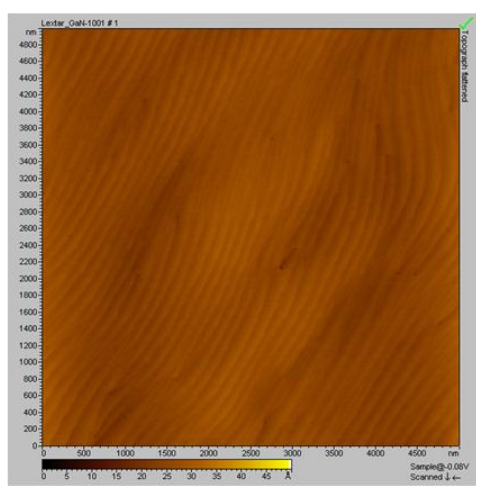

RMS: $0.53 \mathrm{~nm}$

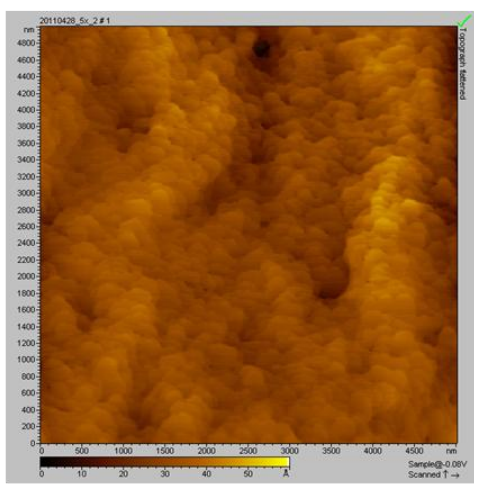

RMS: $1.54 \mathrm{~nm}$

Figure 5-32 - Top Row: AFM images of GaN buffer layers used as substrates for DBR growth. Bottom Row: AFM images of $600 \mathrm{~nm}$ of $n-G a N$ grown on top of 20 period DBRs. 

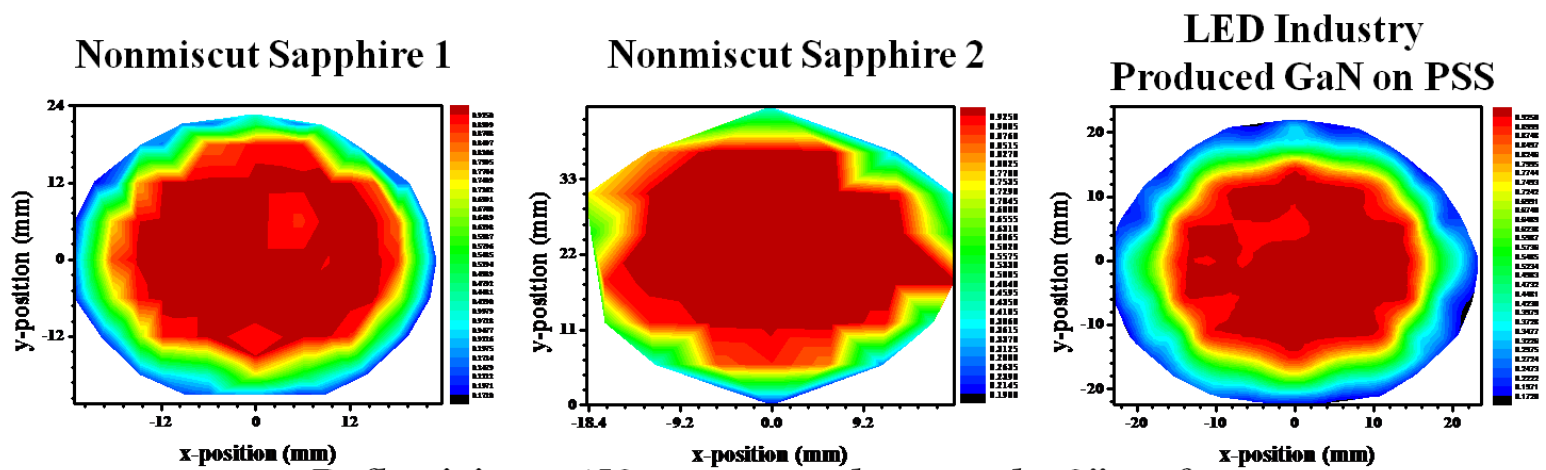

Reflectivity at 452 nm mapped across the 2" wafer
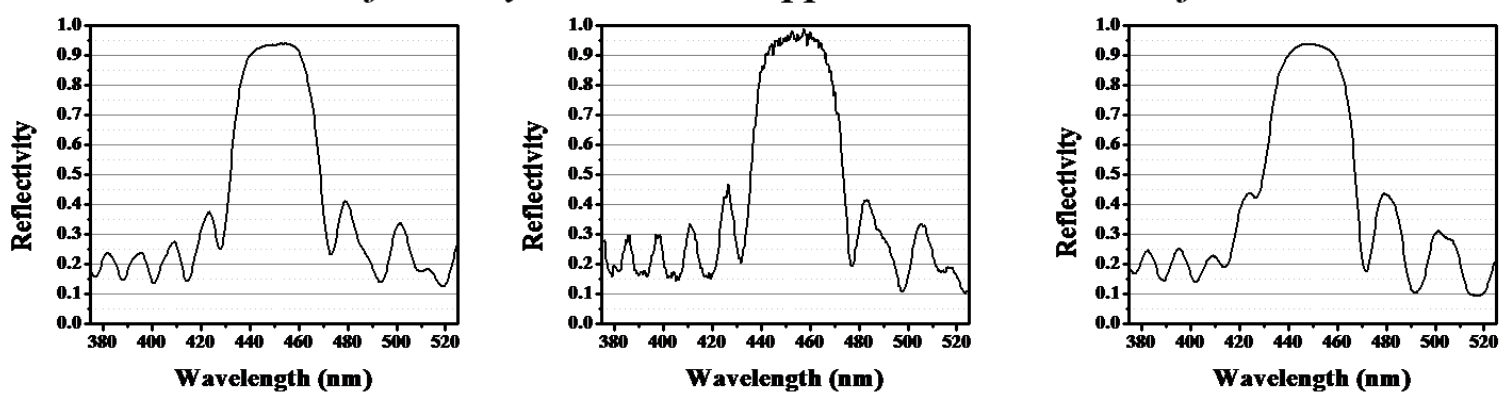

Reflectivity response at the center of the 2" wafer
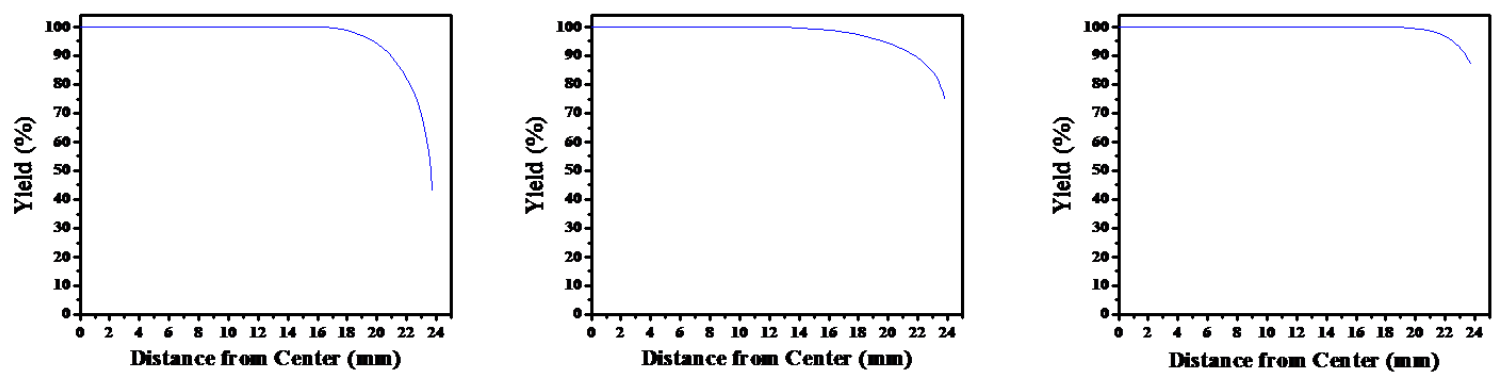

Cracking measured across the 2" wafer

Figure 5-33 - Top Row: Reflectivity map at $452 \mathrm{~nm}$, Second Row: Reflectivity response at the center of the wafer, Bottom Row: Crack free area from the center for the 2" wafer. 


\subsection{Oblique Angles of Incidence}

All of the DBR reflectivity measurements were taken at normal incidence, however from the targeted structure, show in Figure 5-2, it is apparent that the light generated in the active region of the LED may not impinge on the DBR at normal incidence. Therefore, it is necessary to understand how the DBR will respond to light incident at an oblique angle.

Waves propagating through a layered structure are partially transmitted and reflected at each boundary ${ }^{117}$. The overall reflection from the structure is given by the superposition of all these waves. The wave transfer matrix is a useful approach to tracking the many reflected and transmitted waves by relating the electric and magnetic fields at the boundaries between layers ${ }^{89,117}$. The characteristic matrix of the entire structure is found from the multiplication of the individual matrices for each layer making this approach very well suited for superlattice structures. As a result, it is one of the common approaches used for calculating the response of Distributed Bragg Reflectors (DBRs) ${ }^{77}$. For a structure with q dielectric layers, the characteristic matrix is expressed by the following ${ }^{118}$ :

$$
\left[\begin{array}{l}
B \\
C
\end{array}\right]=\prod_{r=1}^{q}\left[\begin{array}{cc}
\cos (\delta) & \frac{i \sin (\delta)}{\eta} \\
i \eta \sin (\delta) & \cos (\delta)
\end{array}\right]\left[\begin{array}{c}
1 \\
\eta_{\text {subs }}
\end{array}\right]
$$

$\mathrm{B}$ and $\mathrm{C}$ are the normalized electric and magnetic field at the initial interface in the structure. $\delta$ is the phase thickness of each layer and is given by:

$$
\delta=\frac{2 \pi n_{r} d \cos \left(\theta_{r}\right)}{\lambda}
$$

$n_{r}$ is the refractive index of the layer, $\theta_{r}$ is the angle in the material, $d$ is the layer thickness, and $\lambda$ is the wavelength. The characteristic admittance, $\eta$, depends on the polarization of the light and is expressed for Transverse Magnetic (TM) and Transverse Electric (TE) polarizations as the following ${ }^{118}$ :

$$
\eta_{T M}=\frac{n \sqrt{\frac{\epsilon_{0}}{\mu_{o}}}}{\cos (\theta)}
$$




$$
\eta_{T E}=n \sqrt{\frac{\epsilon_{o}}{\mu_{o}}} \cos (\theta)
$$

In these equations, $\epsilon_{o}$ is the permittivity of free space $\left(8.8542 \times 10^{-12} \mathrm{C}^{2} / \mathrm{Nm}^{2}\right)$ and $\mu_{\mathrm{o}}$ is the permeability of free space $\left(4 \pi \times 10^{-7} \mathrm{~N} \mathrm{~s}^{2} / \mathrm{C}^{2}\right)$. The optical admittance of the entire structure is expressed by ${ }^{118}$

$$
Y=\frac{C}{B}
$$

Finally, the reflectivity can be calculated from the optical admittance ${ }^{118}$ :

$$
R=\left(\frac{\eta_{o}-Y}{\eta_{o}+Y}\right)\left(\frac{\overline{\eta_{o}-Y}}{\eta_{o}+Y}\right)
$$

In this equation, $\eta_{\mathrm{o}}$ is the admittance of incident medium. Using these relationships, we can determine the influence of the angle of incidence on the reflectivity of an 18 period AlN/GaN DBR structure shown in Figure 5-34. The target wavelength of this structure is $460 \mathrm{~nm}$ and each layer is a quarter-wavelength thick. In this calculation, the refractive indices of GaN and AlN are assumed to be $2.44^{77}$ and $2.1^{119}$ respectively and dispersion has been neglected. Using these values, the AlN layer is $54.76 \mathrm{~nm}$ thick and the GaN layer in the DBR is $47.13 \mathrm{~nm}$ thick. The angle of incidence was taken within a top GaN layer as the DBR structure is typically under the active region in a GaN-based light emitting diode or laser diode. Using Eqs. 5.6 - 5.11, the reflectivity of the DBR has been calculated at angles of incidence, $\theta_{\mathrm{i}}$, ranging from 0 to 80 degrees in which 0 degrees corresponds to normal incidence. Figure 5-35 shows the change in the DBR response for several different incident angles, $\theta_{\mathrm{i}}$. As expected, there is an observable blue-shift in the peak wavelength with increasing angle of incidence. Figure 5-36 contains the reflectivity of the structure at the targeted wavelength of $460 \mathrm{~nm}$ for both TE and TM polarizations. The structure maintains relatively high reflectivity for up to approximately 15 degrees, at which point there is a sudden decrease in reflectivity. Above 58 degrees, the angle of incidence is larger than the critical angle and all the light undergoes total internal reflection at the first GaN/AlN boundary. The reflectivity increases sharply to 1 at this angle for both polarizations. From approximately 15 to 58 degrees, there is negligible reflectance from the DBR at the wavelength for which it was designed to operate. Based on the results in Figure 5-35 
and Figure 5-36, to maximize the response of the DBR over a wider range of angles, the DBR could be designed to reflect a wavelength slightly longer than the targeted emission of the device. The Matlab code for generating these plots is found in Appendix E.

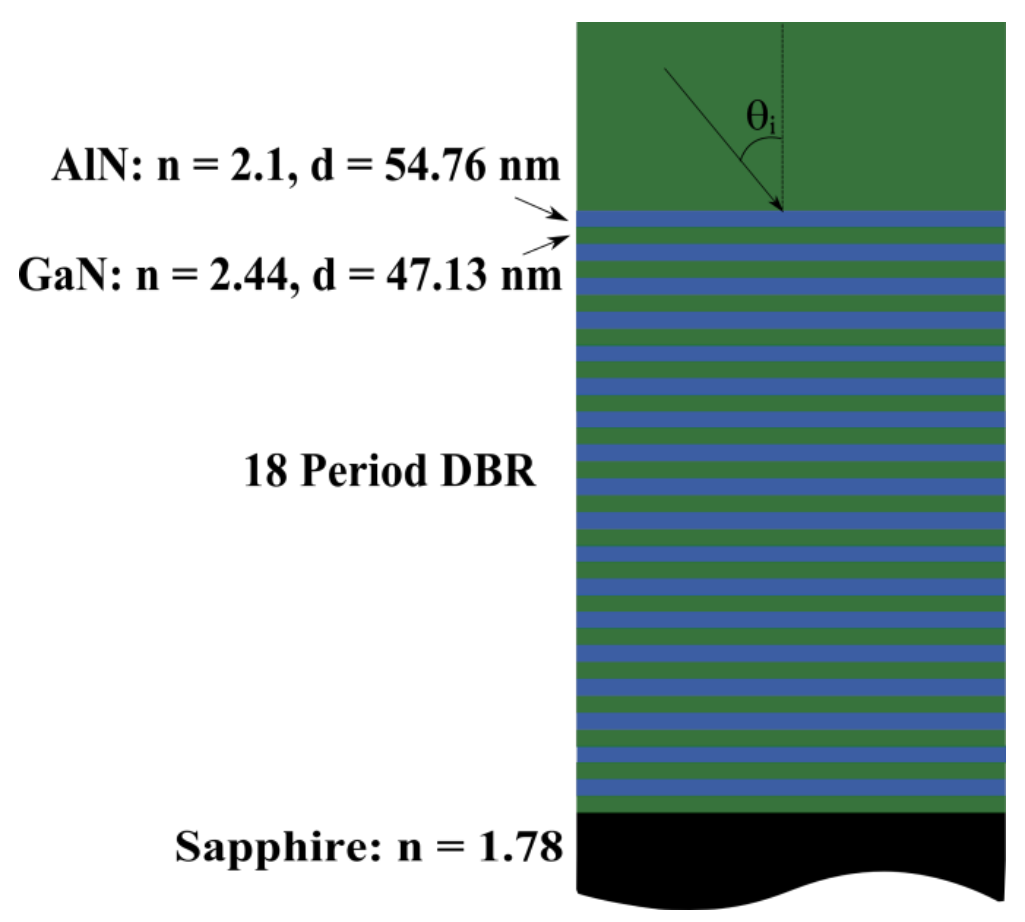

Figure 5-34 - Schematic of 18 period AlN/GaN DBR used in the calculation for DBR response at oblique angles of incidence. 


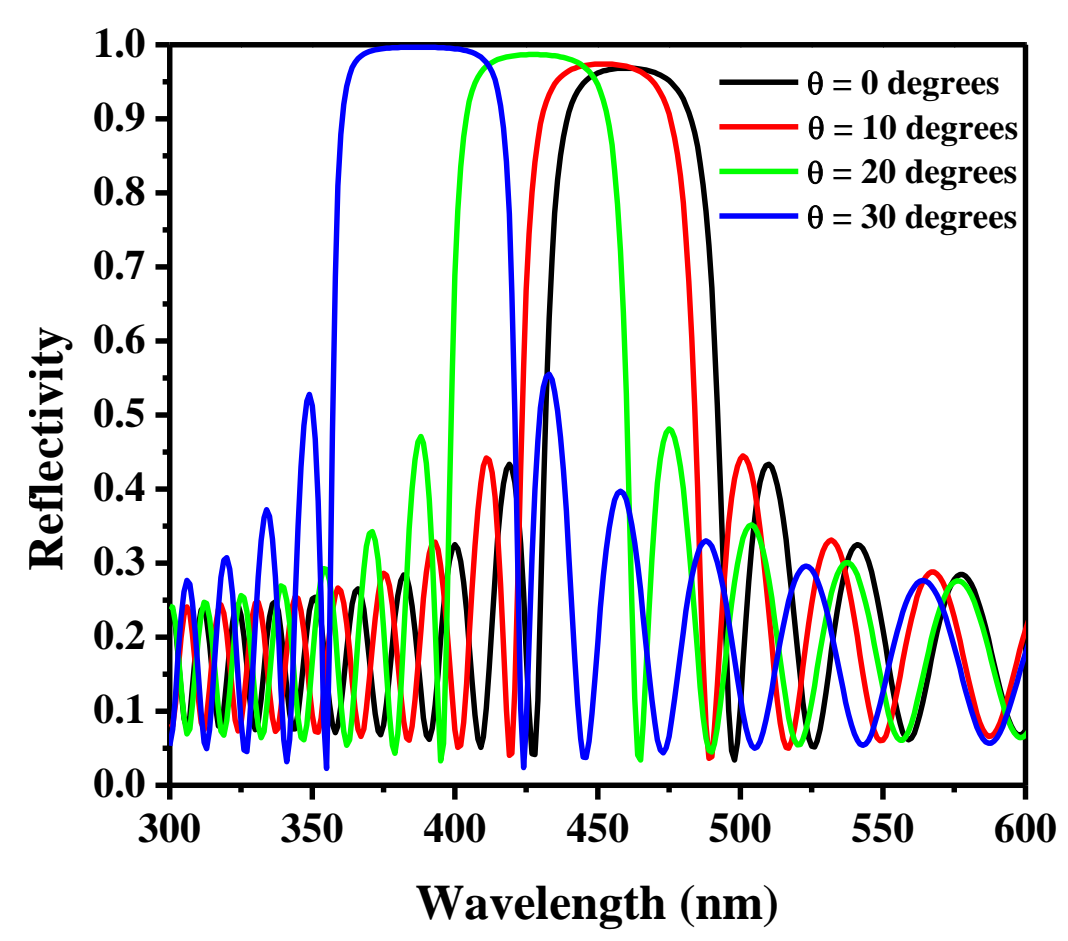

(a)

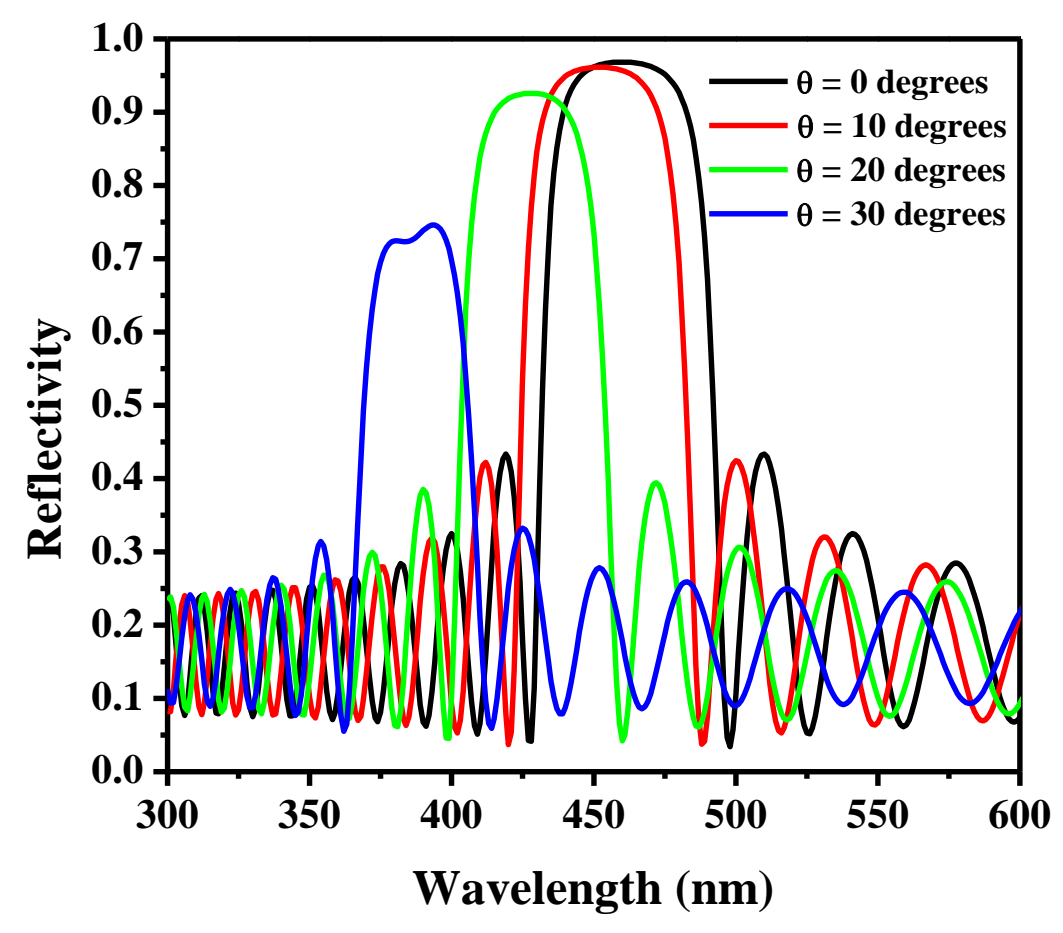

(b)

Figure 5-35 - Reflectivity of 18 period AlN/GaN DBR at oblique angles of incidence for (a)TE and (b) TM polarizations. 


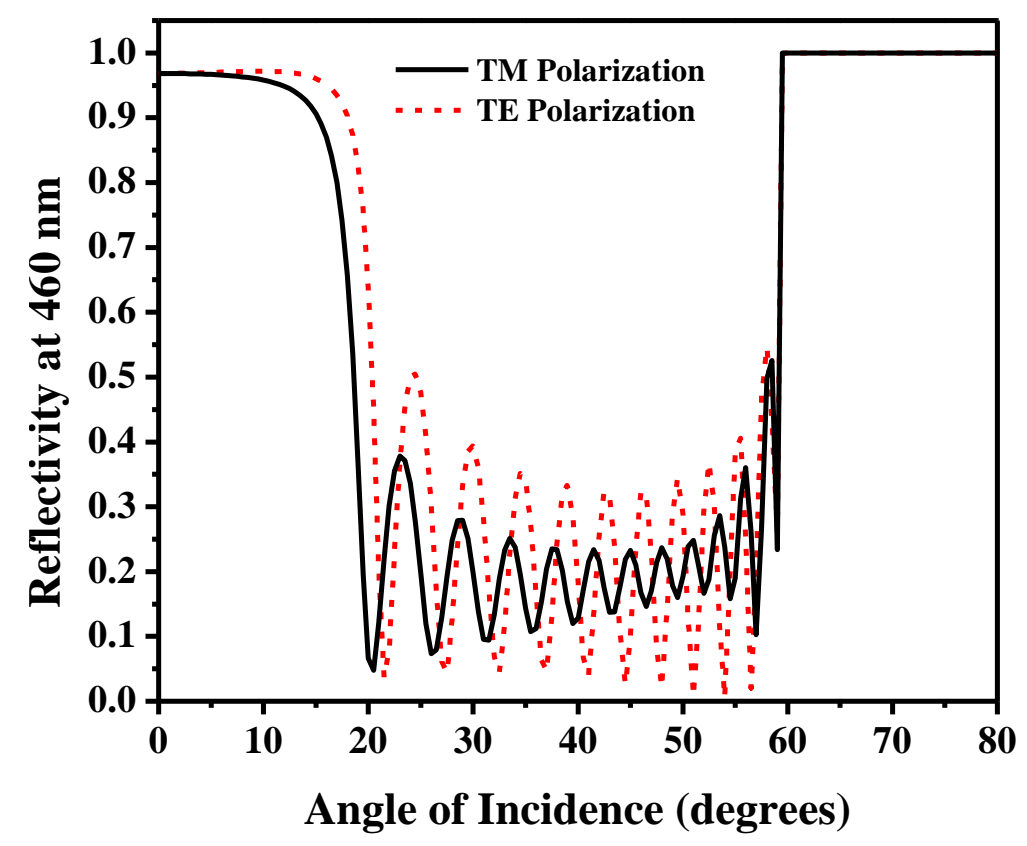

Figure 5-36 - Reflectivity at $460 \mathrm{~nm}$ for an 18 period AlN/GaN DBR for angles of incidence ranging from 0 to 80 degrees.

\subsection{Influence of Strain on Refractive Index and DBR Reflectivity}

Strain induced changes in the refractive index of materials is referred to as the elastooptic effect or photoelastic effect ${ }^{120,121,122}$. In III-Nitride materials, strain can arise from the large lattice and thermal mismatch between the layers and the substrates ${ }^{2}$ and also from different growth techniques and/or material quality ${ }^{123}$. The wide variation in the strain of the materials contributes to the discrepancy in the reported refractive index values of III-Nitride materials ${ }^{121,119}$. As these materials are of significant interest for opto-electronic device fabrication, it is imperative to accurately determine the refractive index and influence of strain for device design.

Refractive index is the ratio of the speed of light in vacuum to the speed of light in the material ${ }^{124}$. It is a material property and is given by the following equation ${ }^{124}$ :

$$
n=\frac{c_{o}}{v}=\sqrt{\frac{\epsilon \mu}{\epsilon_{o} \mu_{o}}}
$$


In this equation $n$ is the refractive index, $c_{o}$ is the speed of light in vacuum $\left(3 \times 10^{8} \mathrm{~m} / \mathrm{s}\right)$, and $v$ is the speed of light in the material of interest. $\epsilon_{o}$ is the permittivity of free space $\left(8.8542 \times 10^{-12} \mathrm{C}^{2} / \mathrm{Nm}^{2}\right)$ and $\epsilon=\epsilon_{o} \epsilon_{r}$ in which $\epsilon_{r}$ is the relative permittivity of the material. Likewise, $\mu_{\mathrm{o}}$ is the permeability of free space $\left(4 \pi \times 10^{-7} \mathrm{~N} \mathrm{~s}^{2} / \mathrm{C}^{2}\right)$ and $\mu=\mu_{\mathrm{o}} \mu_{\mathrm{r}}$ in which $\mu_{\mathrm{r}}$ is the relative permeability of the medium. As this work focuses on nonmagnetic materials, $\mu_{\mathrm{r}}$ is equal to 1 and Eq. 5-12 simplifies to the following:

$$
n=\sqrt{\epsilon_{r}}
$$

The elasto-optic effect relates the change in the refractive index to the strain in a film by the following ${ }^{121,122,125}$ :

$$
\Delta\left(\frac{1}{n^{2}}\right)_{i j}=\sum_{k . l} p_{i j k l} \varepsilon_{k l}
$$

In this equation, $n$ is the refractive index, $p$ is the elasto-optic coefficient tensor, and $\varepsilon$ is the strain tensor. In this form, the elasto-optic coefficient is a dimensionless quantity since both strain and refractive index are dimensionless. The strain is calculated by the deviation of the lattice parameter from the nominal value. To calculate in-plane strain, or strain along the $a$-axis in wurtzite crystals, such as the III-Nitrides, the following equation is used ${ }^{121}$ :

$$
\varepsilon_{x x}=\frac{a_{\mathrm{m}}-a_{\mathrm{o}}}{a_{\mathrm{o}}}
$$

In this expression $a_{\mathrm{m}}$ is the measured $a$-lattice parameter of the film and $a_{\mathrm{o}}$ is the nominal value for a fully relaxed film. In the case of biaxial strain, which is typical in III-Nitrides, it can be assumed that $\varepsilon_{x x}=\varepsilon_{y y}{ }^{121,126}$. Strain in such films is related to the stress by the following equation $^{121}$ :

$$
\sigma_{x x}=M \varepsilon_{x x}
$$

In this equation, $\sigma_{x x}$ is the in-plane stress and $M$ is the biaxial modulus given by ${ }^{121}$

$$
M=S_{11}+S_{12}-2 S_{13}^{2} / S_{33}
$$


$S_{\mathrm{ij}}$ are the elastic coefficients of the materials. Using these relationships, the elasto-optic coefficient can be determined in terms of stress instead of strain and in terms of the dielectric constant instead of the refractive index.

Distributed Bragg Reflectors (DBR) are primary concerned with normal incidence reflectivity, which corresponds to light propagating along the optical axis of the crystal. The relationship between the in-plane strain and the refractive index in this direction is given by the following ${ }^{121}$ :

$$
n\left(\varepsilon_{x x}\right)=n(0)-\frac{1}{2}\left(p_{11}+p_{12}\right) n(0)^{3} \varepsilon_{x x}
$$

In this expression $n\left(\varepsilon_{x x}\right)$ is the refractive index as a function of strain, $n(0)$ is the material's strain-free refractive index, and $p_{11}$ and $p_{12}$ are the components of the elasto-optic tensor that influence the refractive index along this direction. Values for the lattice constants ${ }^{43}$, nominal refractive indices at $460 \mathrm{~nm}^{119}$, and elasto-optic coefficients ${ }^{121,127}$ for AlN and GaN are shown in Table 5-1. There are relatively few reports investigating the elasto-optic parameters of IIINitrides and those shown in Table 5-1 include theoretical and experimental values. The theoretical value for $\mathrm{GaN}$ is approximately 4 times less than the experimental value, indicating that the theoretical values may be strongly underestimated. Since the elasto-optic coefficients for AlN and GaN are similar in value, it can be expected that the true value of $p_{11}+p_{12}$ is approximately 4 times the theoretical value shown in Table 5-1. This assumption will be used for the calculations and simulations performed in this work. Using the values in the Table 5-1 and Eq. 5-18, the influence of strain on the refractive index of GaN and AlN is shown in Figure 5-37.

\subsubsection{Influence of Strain on Reflectivity of Distributed Bragg Reflectors}

For a strained $\mathrm{AlN} / \mathrm{GaN}$ superlattice structure, there are several possible means of relaxation $^{128}$. However, one of the most extreme situations is when there is no relaxation and the superlattice is pseudomorphically grown on a GaN buffer layer. In this case, the AlN layers in the superlattice will be under tensile strain since the lattice parameter of the entire structure is established by underlying buffer layer. In this example, the strain in the AlN layer is approximately 0.025 as calculated from the lattice parameters in Table 5-1 and Eq. 5-15. The 
Table 5-1 - Constants for AlN and GaN including a and c-lattice parameters ${ }^{43}$, refractive index ${ }^{119}$, and elasto-optic coefficients ${ }^{121,127}$.

\begin{tabular}{|c|c|c|}
\hline & AIN & GaN \\
\hline$a_{\mathrm{o}}(\AA)$ & 3.11 & 3.189 \\
\hline$c_{\mathrm{o}}(\AA)$ & 4.98 & 5.185 \\
\hline$n(460 \mathrm{~nm})$ & 2.1 & 2.44 \\
\hline$p_{11}+p_{12}$ & -0.127 (Theoretical) $^{127}$ & -0.109 (Theoretical) $^{127}$ \\
& & -0.460 (Experimental) $^{121}$ \\
\hline
\end{tabular}

change in the AIN refractive index for this strain value is approximately $\Delta n=0.056$. Performing a simplified simulation of an 18 period AlN/GaN DBR using the transmission matrix ${ }^{77}$ method allows us to determine the impact of the refractive index increase on the peak reflectivity. As can been seen in Figure 5-38, the increase in the refractive index of AlN due to tensile strain would result in a decrease in reflectivity around $2 \%$. The simplified simulation shown in Figure 5-38 neglects the dispersion of the refractive index. As the refractive index contrast between AlN and GaN decreases with increasing strain, there is also an observable decrease in the bandwidth of the structure. As mentioned previously, this is an extreme example. It is expected that AIN layers in a DBR reflecting in the visible regime (thickness $>48 \mathrm{~nm}$ ) would relax through the formation of defects, surface roughness, or cracks ${ }^{67}$. 

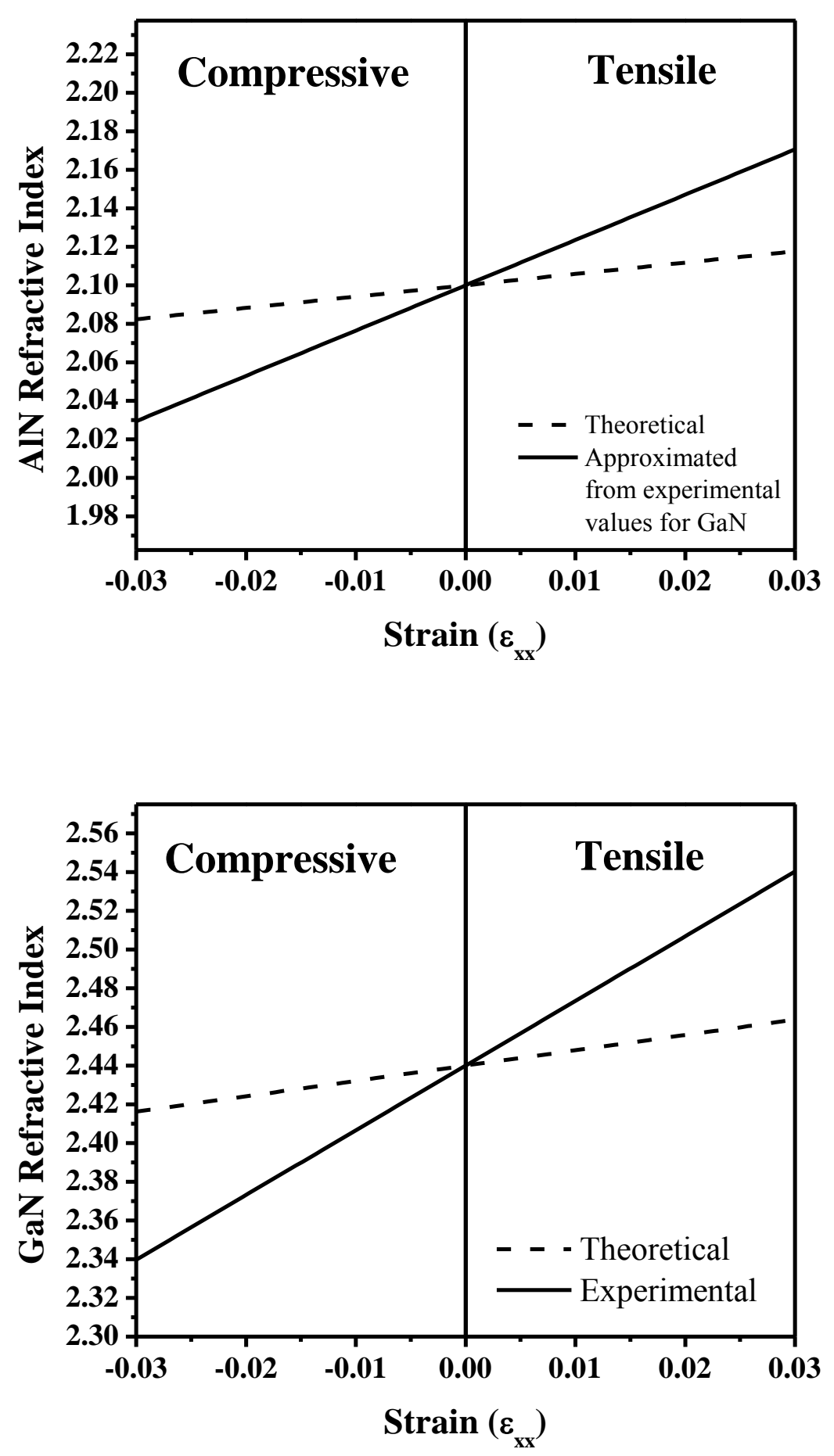

Figure 5-37 - Refractive index of AlN (left) and GaN (right) at $460 \mathrm{~nm}$ as a function of in-plane strain calculated from Eq. 5-18 using the theoretical and experimental values of elasto-optic coefficient shown in Table 5-1. Note: Approximated value of elasto-optic coefficient for AlN was estimated to be $p_{11}+p_{12}=0.508$. 


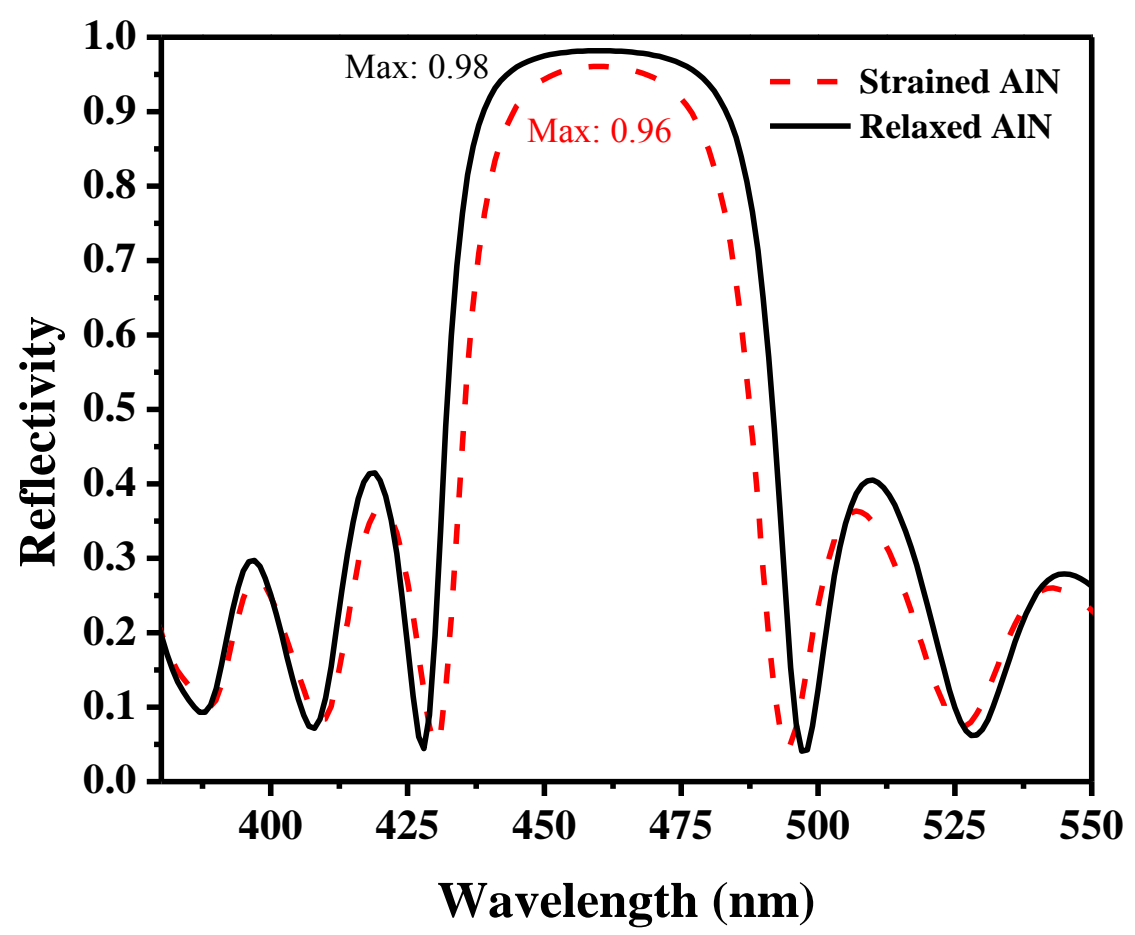

Figure 5-38 - Reflectivity spectra of 18 period AlN/GaN DBR. GaN refractive index was 2.44 and AlN refractive index was 2.1 and 2.156 for relaxed and strained simulations respectively.

\subsubsection{Measurement of Refractive Index}

Common techniques used to determine the refractive index of materials include $\operatorname{trasmission}^{88}$, prism coupling ${ }^{121,129}$, and spectroscopic ellipsometry ${ }^{130}$. Transmission measurements allow the refractive index to be determined based on the oscillations arising from optical interference ${ }^{88}$. It is a useful technique for extracting refractive index and film thickness, however, it is not suitable for very thin films or complex structures. Prism coupling is another technique that is ideal for planar films ${ }^{121,129}$. In this method a prism is held against the sample surface and used to couple laser light to the sample. The guided modes are identified by changing the angle between the prism and in the incident laser ${ }^{121}$. As with transmission measurements, this technique is not suitable for very complex structures. The third technique commonly used of the identification of refractive indices and optical properties is the spectroscopic ellipsometry ${ }^{130}$. This technique is based upon measuring the change in the polarization of light as it interacts with a material. Ellipsometry allows for the modeling of 
complex optical systems with multiple layers and materials and may be well suited for DBR modeling.

\subsubsection{Measurement of Strain}

In order to anticipate the change in refractive index, the strain in the films needs to be determined. One of the more common means to measure strain is the use of X-Ray Diffraction (XRD). On-axis XRD measurements allow the out-of-plane (c) lattice parameter to be determined while off-axis scans determine the in-plane $(a)$ parameter $^{87}$. The deviation of the measured parameter from the well documented nominal lattice parameters allow the strain to be calculated from Eq. 5-15. Other techniques for measuring the strain include the use of photoluminescence or reflectivity measurements to determine the exciton energies ${ }^{121,131}$. Shifts in these energies can be attributed to strain and this method has been used in the III-Nitrides to measure strain in individual layers. 


\section{CHA P T E R 6 - I N T E GRA T I O N O F D B R S W I TH L I GH T E M I T T I N G D I O D E S}

\subsection{Introduction}

In this chapter, the results of the Light Emitting Diode (LED) and DBR integration are presented $^{132}$. As discussed in the previous chapters, the target for this work is to integrate an LED on a DBR substrate to improve light extraction. The growth as well as the characterization of the devices is discussed.

\subsection{Experimental Method}

$\mathrm{In}_{x} \mathrm{Ga}_{1-x} \mathrm{~N} / \mathrm{GaN}$ based LEDs were grown on a GaN epilayers, 6 period DBR, and 12 period DBR. All DBRs were digital $\mathrm{Al}_{x} \mathrm{Ga}_{1-x} \mathrm{~N} / \mathrm{GaN}$ quarter wavelength DBRS as described in the previous chapter. The total structure is shown in Figure 6-1 and consists of a $30 \mathrm{~nm}$ AlN buffer layer, $1.8 \mu \mathrm{m}$ GaN epilayer, digital $\mathrm{Al}_{x} \mathrm{Ga}_{1-x} \mathrm{~N} / \mathrm{GaN} \mathrm{DBR}, 1.5 \mu \mathrm{m}$ Si-doped GaN epilayer, five period $\operatorname{In}_{x} \mathrm{Ga}_{1-x} \mathrm{~N} / \mathrm{GaN}$ multi quantum wells (MQW) targeted for emission at $\sim 440 \mathrm{~nm}, 10 \mathrm{~nm}$

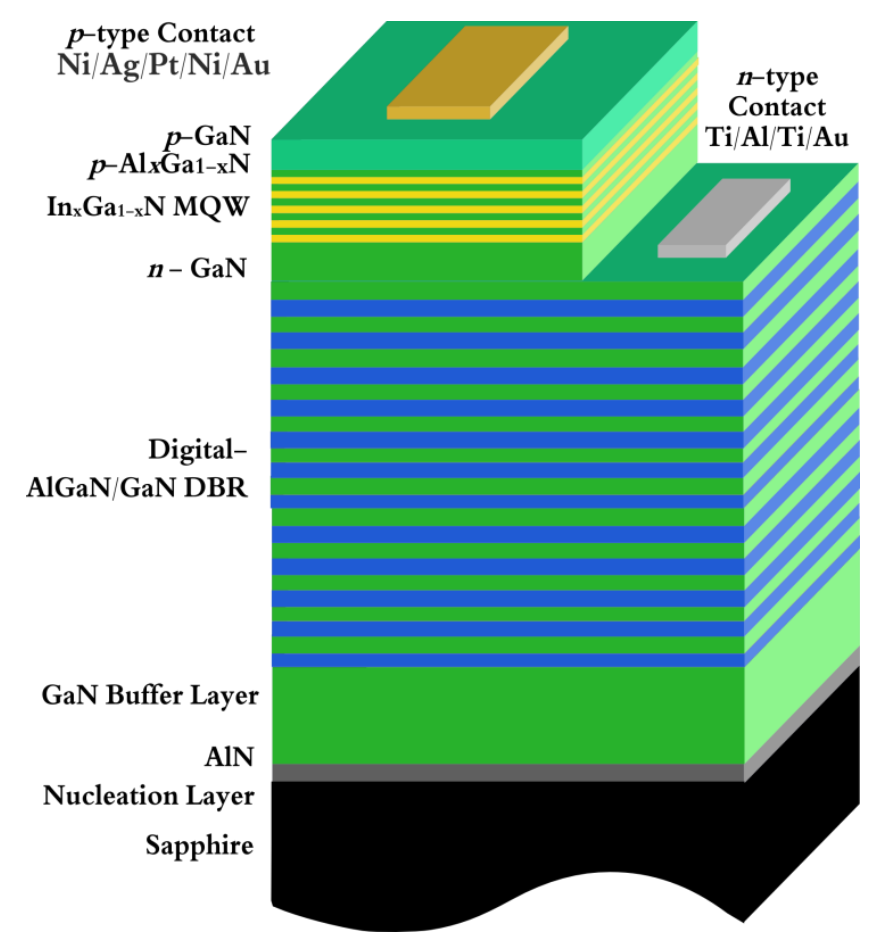

Figure 6-1 - Schematic of the LED structure. 
Mg-doped $\mathrm{Al}_{x} \mathrm{Ga}_{1-x} \mathrm{~N}$ electron blocking layer, and a $70 \mathrm{~nm} \mathrm{Mg-doped} \mathrm{GaN}$ epilayer. Prior to LED growth, the reflectivity of 6 period and 12 period digital $\mathrm{Al}_{x} \mathrm{Ga}_{1-x} \mathrm{~N} / \mathrm{GaN}$ DBR substrate was measured and is shown in Figure 6-3. The 6 and 12 period DBR exhibited $60 \%$ and $90 \%$ reflectivity, respectively. After the LED growth, the sample was annealed at $800{ }^{\circ} \mathrm{C}$ for 5 minutes to activate the $\mathrm{Mg}$ dopants. The LED mesas were fabricated using standard UV photolithography patterning and inductively coupled plasma reactive ion etching. The $n$-type and p-type contacts consisted of $\mathrm{Ti} / \mathrm{Al} / \mathrm{Ti} / \mathrm{Au}$ and $\mathrm{Ni} / \mathrm{Ag} / \mathrm{Pt} / \mathrm{Ni} / \mathrm{Au}$ metal stacks deposited via e-beam evaporation. The $p$-type contact was a high reflectivity Ag-based contact developed in order to provide a top side mirror ${ }^{133}$. The final metallization scheme used was a $\mathrm{Ni}(1 \mathrm{~nm}) / \mathrm{Ag}(150$ $\mathrm{nm}) / \mathrm{Pt}(50 \mathrm{~nm}) / \mathrm{Ni}(20 \mathrm{~nm}) / \mathrm{Au}(50 \mathrm{~nm})$ stack followed by an anneal at $430{ }^{\circ} \mathrm{C}$. Characterization of the samples included cross sectional SEM imaging, XRD, I-V, and EL. Image processing techniques were employed to evaluate the emission intensity from the topside of the LEDs

\subsection{Characterization}

Cross sectional SEM images were taken to verify the structure and are shown in Figure 6-3. XRD measurements were taken to measure average indium composition of the MQWs and the period of the superlattice. The MQW growths on the three substrates were similar and had periodicities around $13 \mathrm{~nm}$ and an average $\mathrm{InN}$ mole fraction around 0.04 as extracted from the (0002) scan as shown in Figure 6-4. Standard I-V measurements were taken and used to determine the turn on voltage as well as the series resistance. Figure 6-7 shows the I-V characteristics of the two LEDs on DBR substrates and the LED on the GaN substrate. The LEDs on DBRs had a turn on voltage of $3.0 \mathrm{~V}$ and $2.89 \mathrm{~V}$ for the growths on the 6 period and 12 period DBR, respectively. This is slightly higher than the $2.68 \mathrm{~V}$ turn on voltage of the LED on the GaN epilayer. The series resistance was determined to be $42.18 \Omega, 40.93 \Omega, 47.85 \Omega$ for the LED on GaN, the LED on the 6 period DBR, and the LED on the 12 period DBR, respectively. Figure 6-6 shows an optical image of the 12 period DBR with LED under forward bias and also the final fabricated structures on the chip. The structure consists of a $400 \mu \mathrm{m}$ diameter circular mesa with a centered $p$-type contact. The $n$-type contact formed a ring around the mesa. 


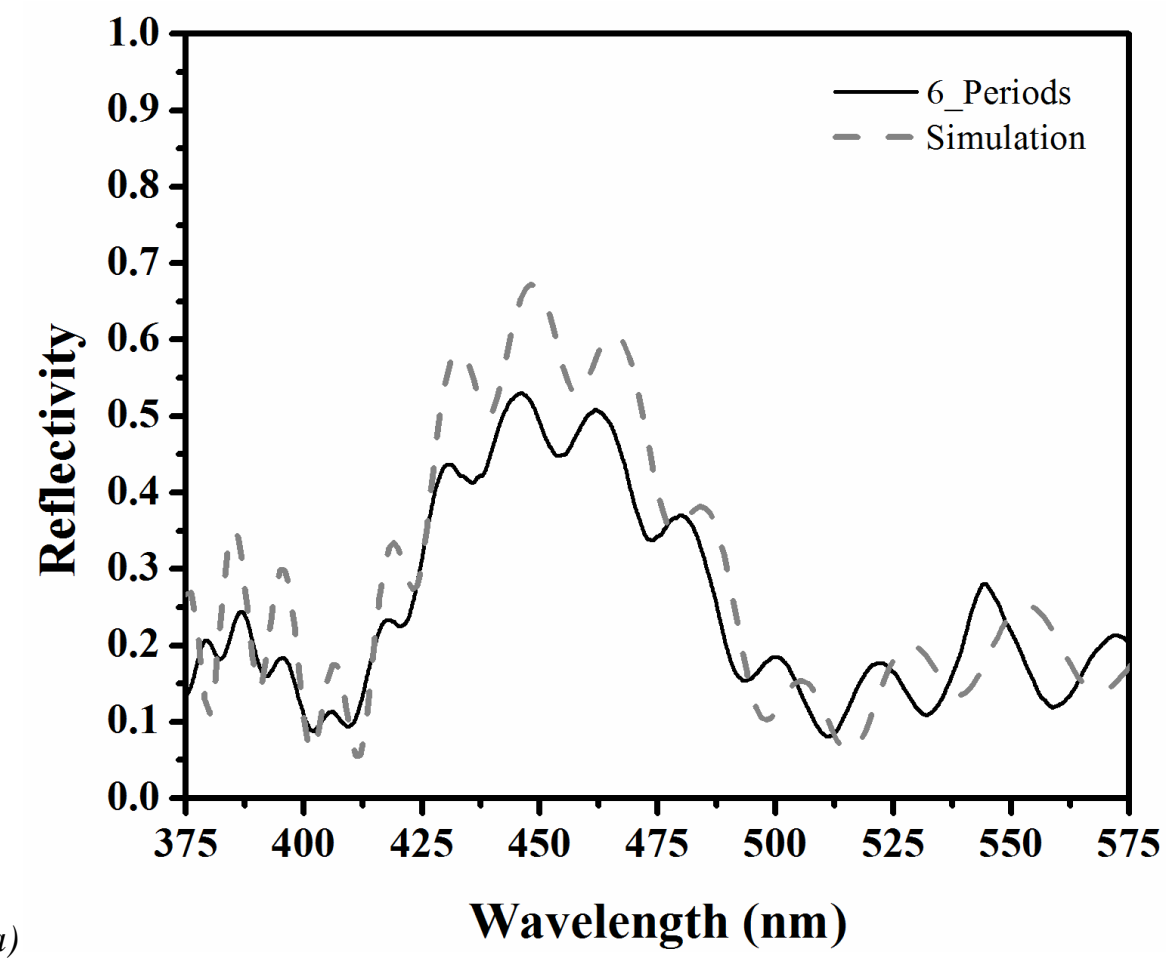

(a)

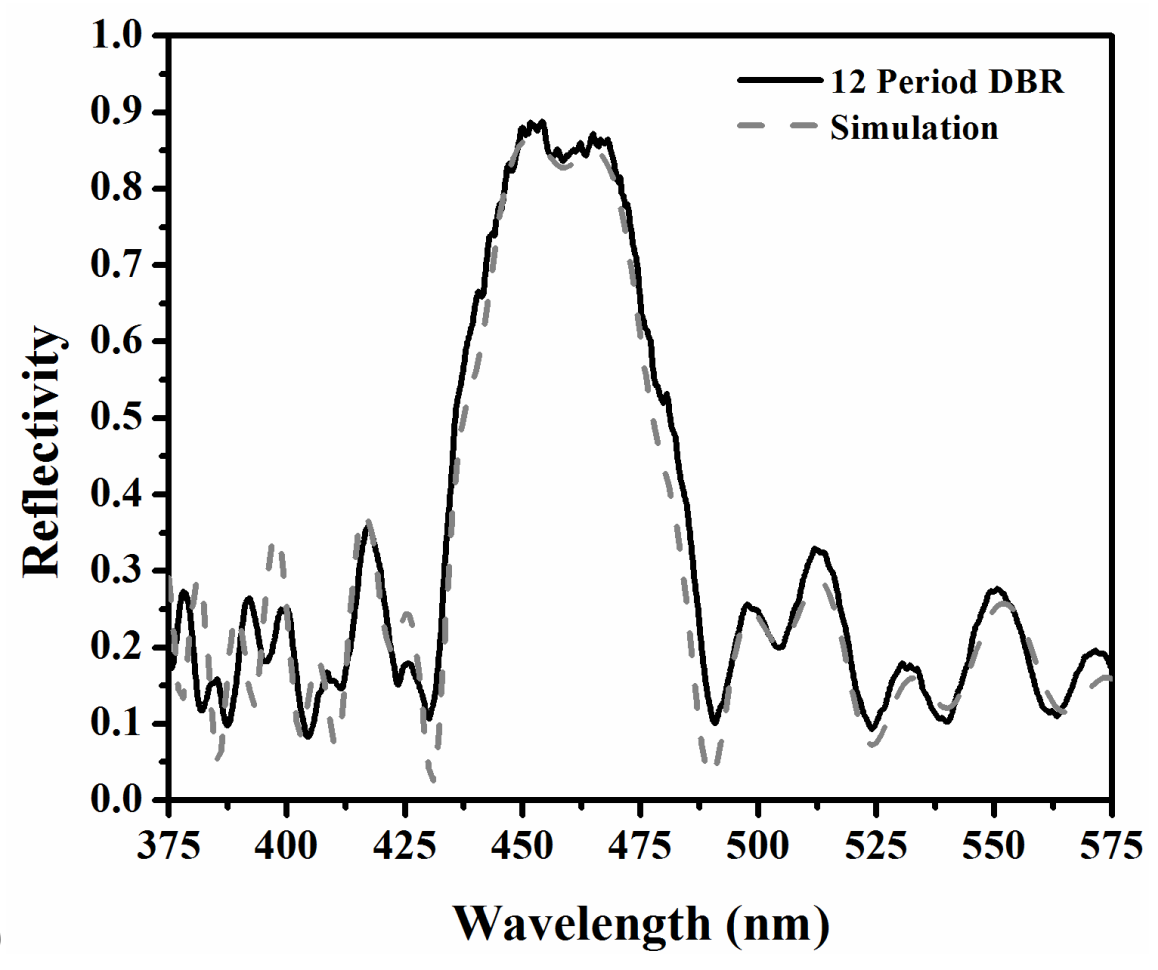

Figure 6-2 - Reflectivity of the DBR substrates integrated with LED. (a) Six period DBR and (b) 12 period DBR reflectivity spectra. 


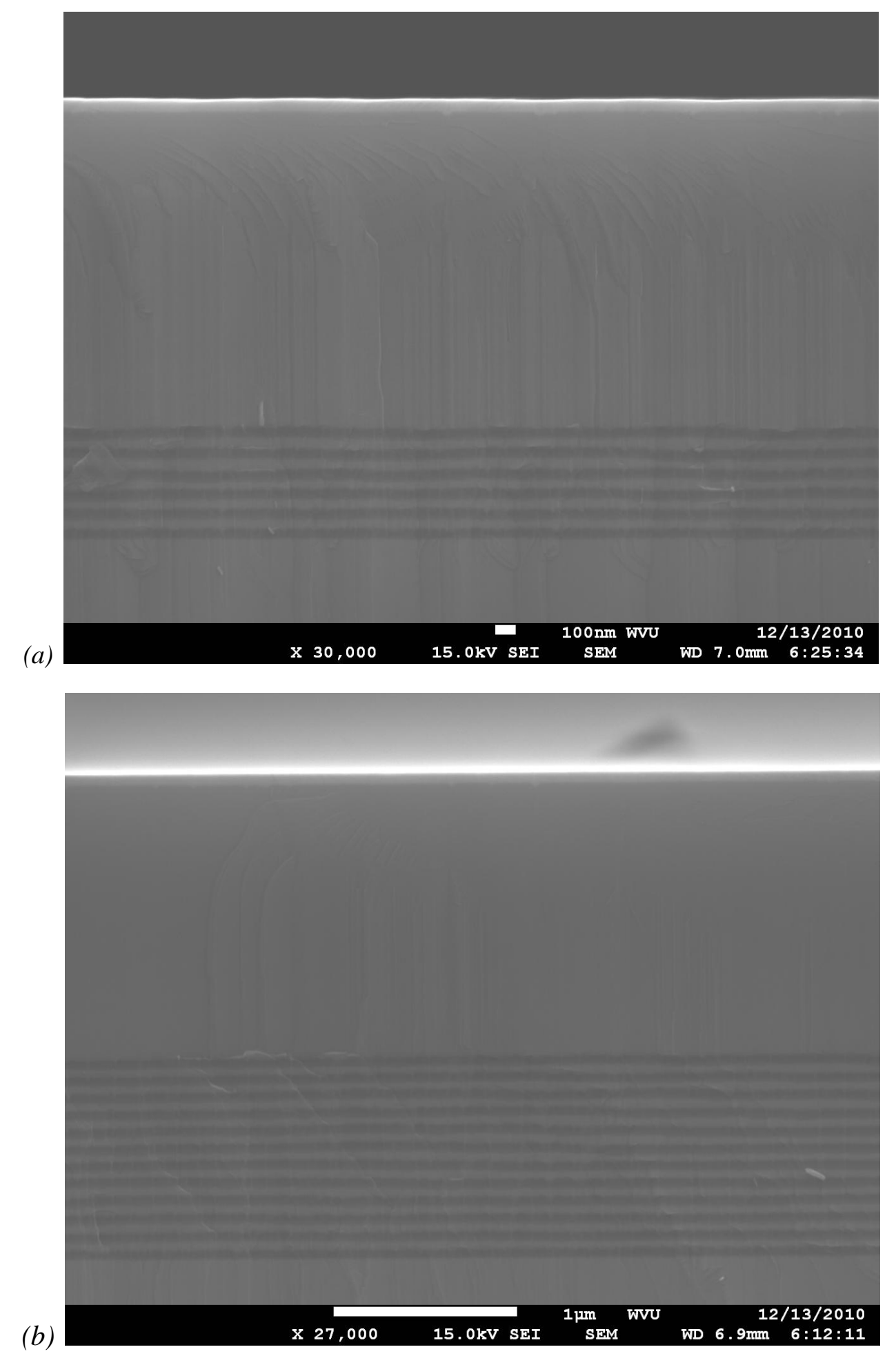

Figure 6-3 - Cross sectional SEM of LED on (a) 6 period DBR and (b) 12 period DBR. 


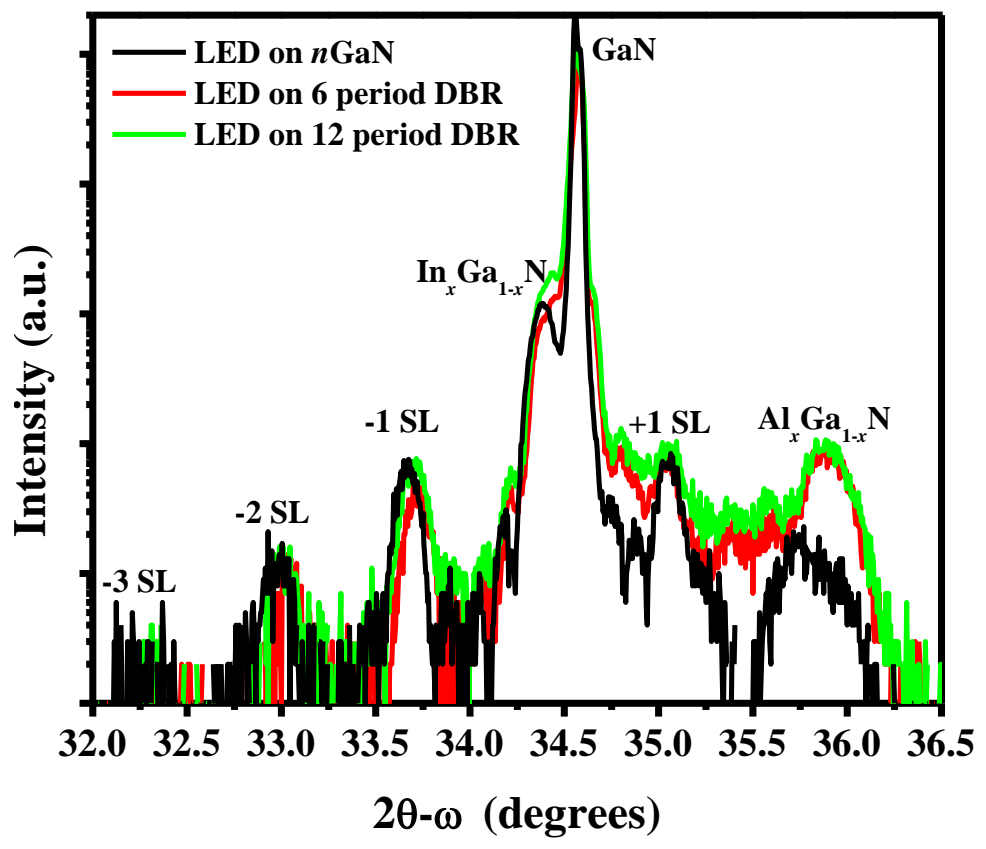

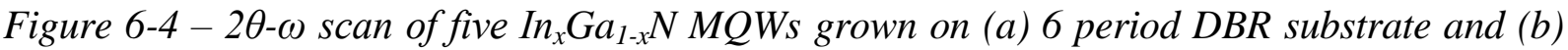
12 period DBR substrate.

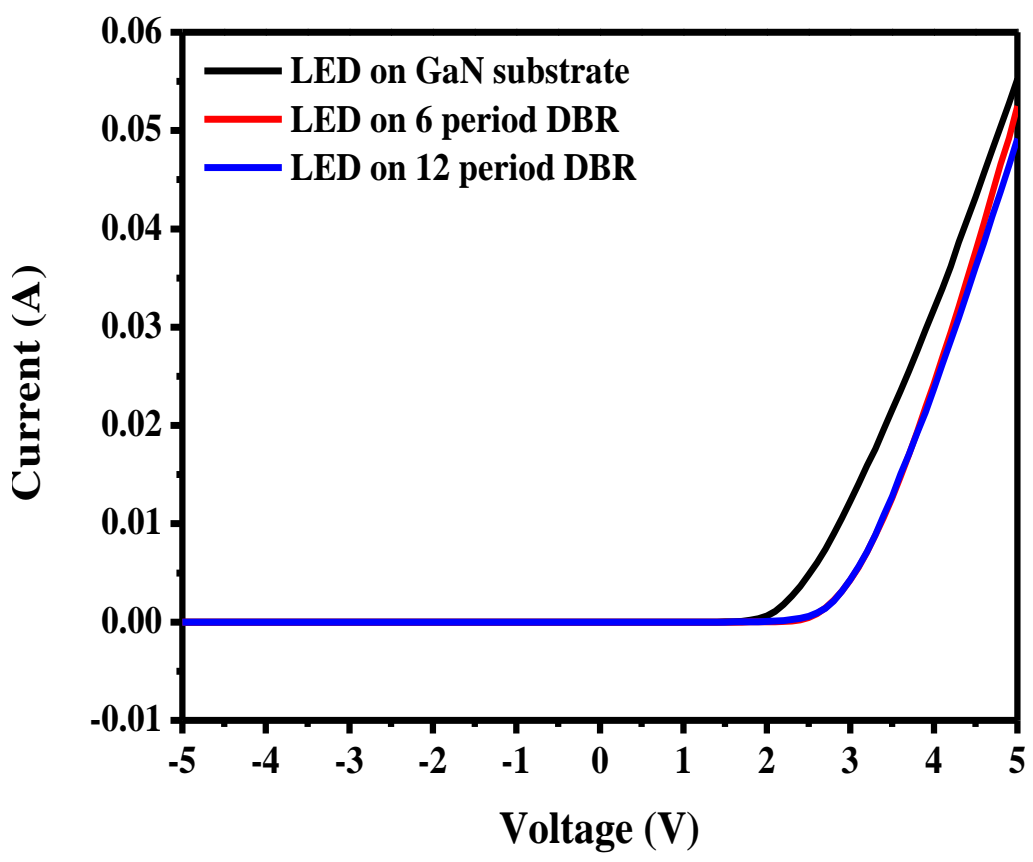

Figure 6-5 - I-V characteristics for LED on DBR samples. 


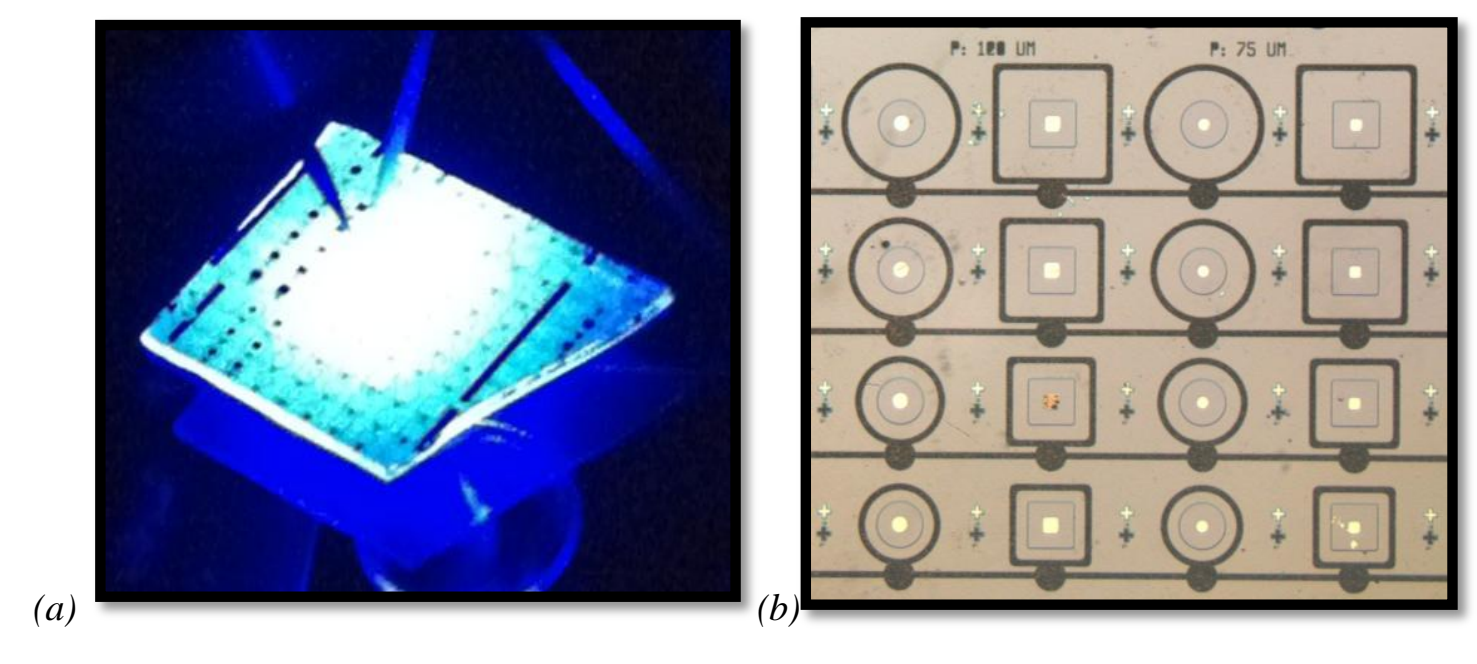

Figure 6-6 - (a) Optical Image of LED on 12 period DBRand (b) optical image of fabricated devices.

Figure 6-7 shows the room temperature EL spectra of the 3 LEDs. The emission peak wavelength was located around $441 \mathrm{~nm}$ and the FWHM of the emission was $17.4 \mathrm{~nm}$ and 28.1 $\mathrm{nm}$ for LED on the 6 period DBR and the LED on 12 period DBR, respectively. In addition, there are strong vertical modes that emerge in the EL spectra. These modes are formed by vertical distances between layers with differing refractive indices.

Optical images were taken from the top of the LEDs in order to evaluate the extraction intensity. Figure 6-8 shows the normalized light intensity extracted from these images for different samples. In this figure, the sample "LED" refers to a LED on a GaN epilayer. The sample "LED w/Mirror" refers to the LED on a GaN epilayer placed on top of an Al coated pyrex mirror. This was done to simulate the metallization of the back side of the sapphire wafer which is often done in the LED industry. The Al coated mirror has the same reflectivity response that was shown in Figure 3-21. The sample "LED w/ 6 period DBR" refers to the LED grown on the 6 period DBR and likewise, the sample "LED w/ 12 period DBR" refers to the LED grown on the 12 period DBR. The mirror and the six period DBR had approximately the same effect and significantly increase the light emitted from the top of the device. Using a 12 period DBR increase the extracted light by approximately $25 \%$ more.

In summary, digital alloy based DBRs have been demonstrated a suitable substrate for LED growth. The quality, composition, and periodicity of the $\operatorname{In}_{x} \mathrm{Ga}_{1-x} \mathrm{~N} / \mathrm{GaN}$ based LEDs 


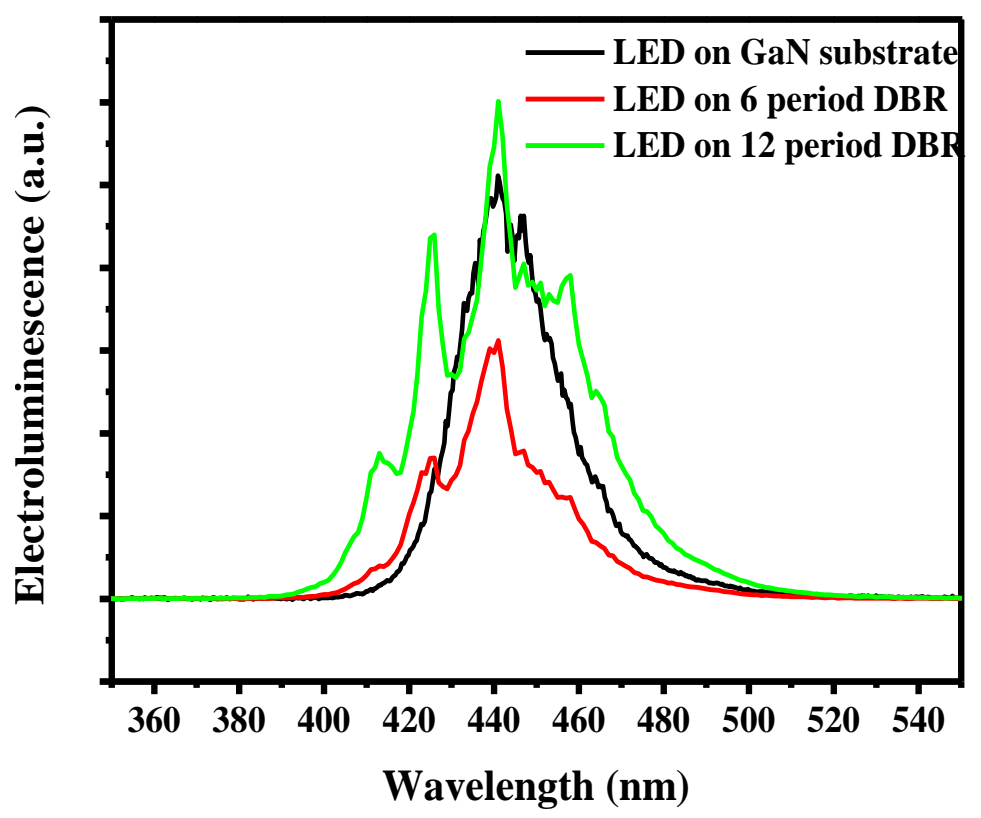

Figure 6-7 - EL spectra of LEDs with and without integrated DBRs.

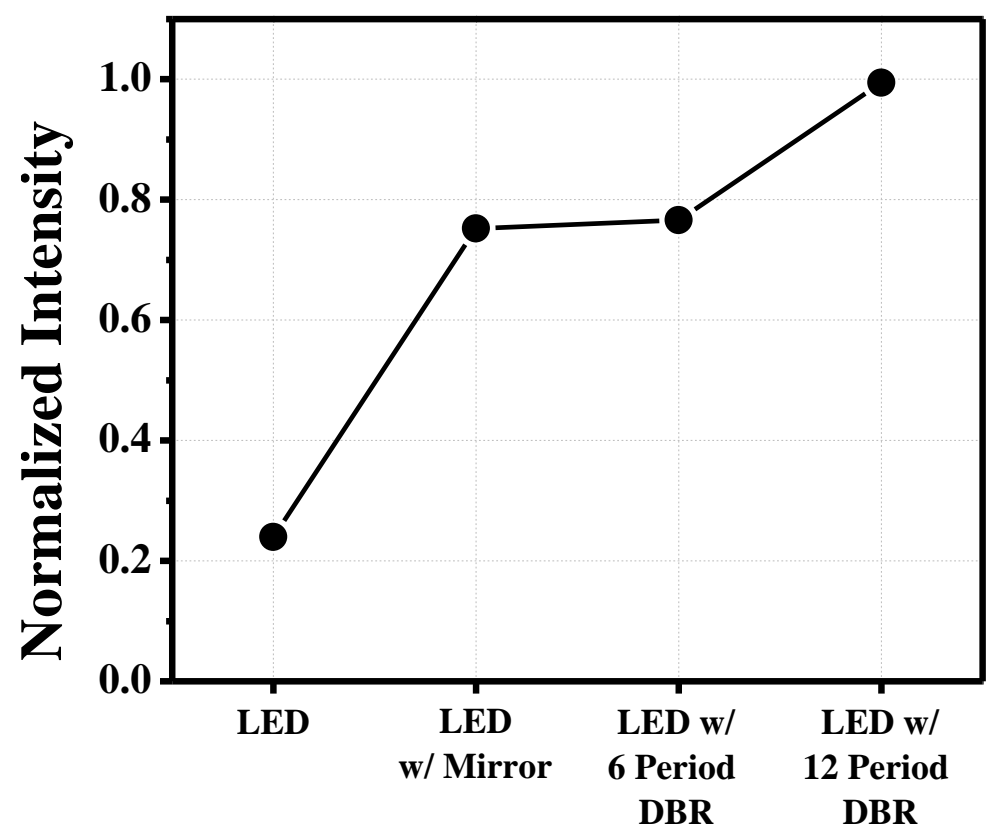

Figure 6-8-Normalized LED intensity extracted from optical images. 
grown on DBRs were similar to those grown on GaN epilayers. DBRs with reflectivity responses ranging from $\sim 60 \%$ to $\sim 90 \%$ have been integrated with $440 \mathrm{~nm}$ emitters and the light extraction has been measured. A considerable increase in light extraction has been measured from the top of the devices as compared to planar LEDs. 


\section{H A P T E R $7-$ C U R V E D L E D S}

\subsection{Introduction}

Two areas of interest in LED development include engineering the radiation profile and increasing the efficiency. Depending on the LED application, including solid state lighting, full color displays, automotive lighting, and device lighting ${ }^{134}$, either narrow or broad radiation patterns are desirable. For example, in solid state lighting applications, broad patterns are used to create uniform lighting from most angles. However, for short distance fiber optic communications, narrow emission patterns are necessary to improve fiber coupling efficiency ${ }^{135}$. Furthermore, despite commercial success, nitride based LEDs are still hindered by limited efficiency. Part of this inefficiency arises from limited light extraction as nitride based devices have a narrow light escape cone and part from limited internal quantum efficiency due to material defects and polarization effects ${ }^{134}$. As a result, considerable research efforts have focused on developing techniques to modify the emission pattern and improving the efficiency of nitride based LEDs including, but not limited to, micro-lens ${ }^{136,137}$, photonic crystals ${ }^{134,138}$, resonant cavity devices ${ }^{135}$, and three-dimensional faceted structures ${ }^{139,140}$. Three-dimensional structures fabricated via Selective Area Growth (SAG) have been investigated for many years as a means for growing on semi-polar planes ${ }^{139,140}$. It has been well documented that growth on semi-polar planes reduce the internal electric field resulting from the spontaneous and piezoelectric polarization inherent in III-Nitride materials and is often pursued for the fabrication of high efficiency devices ${ }^{139,141}$.

In this work, the feasibility of fabricating emitters on curved surfaces has been demonstrated. The growth kinetics of the SAG of GaN have been utilized to fabricate curved surfaces. Indium Gallium Nitride $\left(\operatorname{In}_{1-x} \mathrm{Ga}_{x} \mathrm{~N}\right)$ based Multi-Quantum Well (MQW) LED structures have been grown on the curved surface and devices have been fabricated using standard micro-fabrication techniques ${ }^{142}$. The electrical and optical characteristics of these structures are discussed. This technique present the potential for both engineering the emission pattern and also fabrication of devices on semi-polar planes. 


\subsection{Experimental Methods}

All materials were grown via MOVPE in the Aixtron horizontal reactor. TMGa, TMAl, TMIn, TEGa, $\mathrm{Cp}_{2} \mathrm{Mg}$, and $\mathrm{NH}_{3}$ were used as the precursors. Approximately $2 \mu \mathrm{m}$ GaN buffer layers were grown on $c$-plane sapphire substrates using an AIN buffer layer. Silicon oxide and silicon nitride masks were deposited via Plasma Enhanced Chemical Vapor Deposition (PECVD) using an Oxford Plasma Lab 80 Plus system. Standard ultra violet photolithography techniques were used create a striped pattern along $<1 \overline{1} 00>$ direction of the GaN. This direction was used as the geometry of SAG with stripes along this direction is easily controlled by the growth conditions ${ }^{143}$. This striped pattern had window openings ranging from $80 \mu \mathrm{m}-$ $110 \mu \mathrm{m}$ with a spacing of $400 \mu \mathrm{m}$. The photoresist pattern was transferred to the dielectric mask by wet etching in buffered oxide etchant. The patterned substrate, as shown in Figure 7-1, was then returned to the MOVPE reactor for SAG. The surface kinetics on the dielectric materials prevent the deposition of $\mathrm{GaN}$ on the mask where as the growth in the expose stripe is allowed $^{143,144}$. As described previously for SAG and ELOG, the species on the surface of the mask will diffuse in the gas phase or migrate on the surface of the mask to the opening. By changing the mask dimensions there is localized engineering of the V/III ratio during growth. In this work, relatively large stripe widths and window openings were used such that the species diffusing from the mask would be incorporated along the edge of the stripe as compared to the center area giving rise to a curved cross section ${ }^{144}$ along the stripe as illustrated in Figure 7-1b. The SAG was performed at a reactor temperature of $1000{ }^{\circ} \mathrm{C}$ and pressure of $200 \mathrm{mbar}$. The TMGa flow rate was $120 \mu \mathrm{mol} / \mathrm{min}$ and V/III ratios of 560 and 940 were investigated. The dielectric mask was then removed by wet etching and a simple LED structure was grown. This included five $\operatorname{In}_{x} \mathrm{Ga}_{1-x} \mathrm{~N} / \mathrm{GaN}$ multi-quantum wells (MQWs) followed by a $10 \mathrm{~nm} p-\mathrm{Al}_{x} \mathrm{Ga}_{1-x} \mathrm{~N}$ layer and a $70 \mathrm{~nm}$-GaN layer. The TMIn flow rate for the MQW was $6 \mu \mathrm{mol} / \mathrm{min}$, TEGa was $21 \mu \mathrm{mol} / \mathrm{min}$, and the $\mathrm{V} / \mathrm{III}$ ratio was 4800 . The $p-\mathrm{GaN}$ was grown at $950{ }^{\circ} \mathrm{C}$ using $66 \mu \mathrm{mol} / \mathrm{min}$ of TMGa, $1.2 \mu \mathrm{mol} / \mathrm{min}$ of $\mathrm{Cp}_{2} \mathrm{Mg}$, and a V/III ratio of 1000 . The most basic LED structure was targeted in this work in order to eliminate the challenges associated with growth on a non-planar surface.

In order to fabricate the LED structure, the flow process shown in Figure 7-2 was followed. First a circular $p$-type contact was deposited and then the LED mesa was etched as 


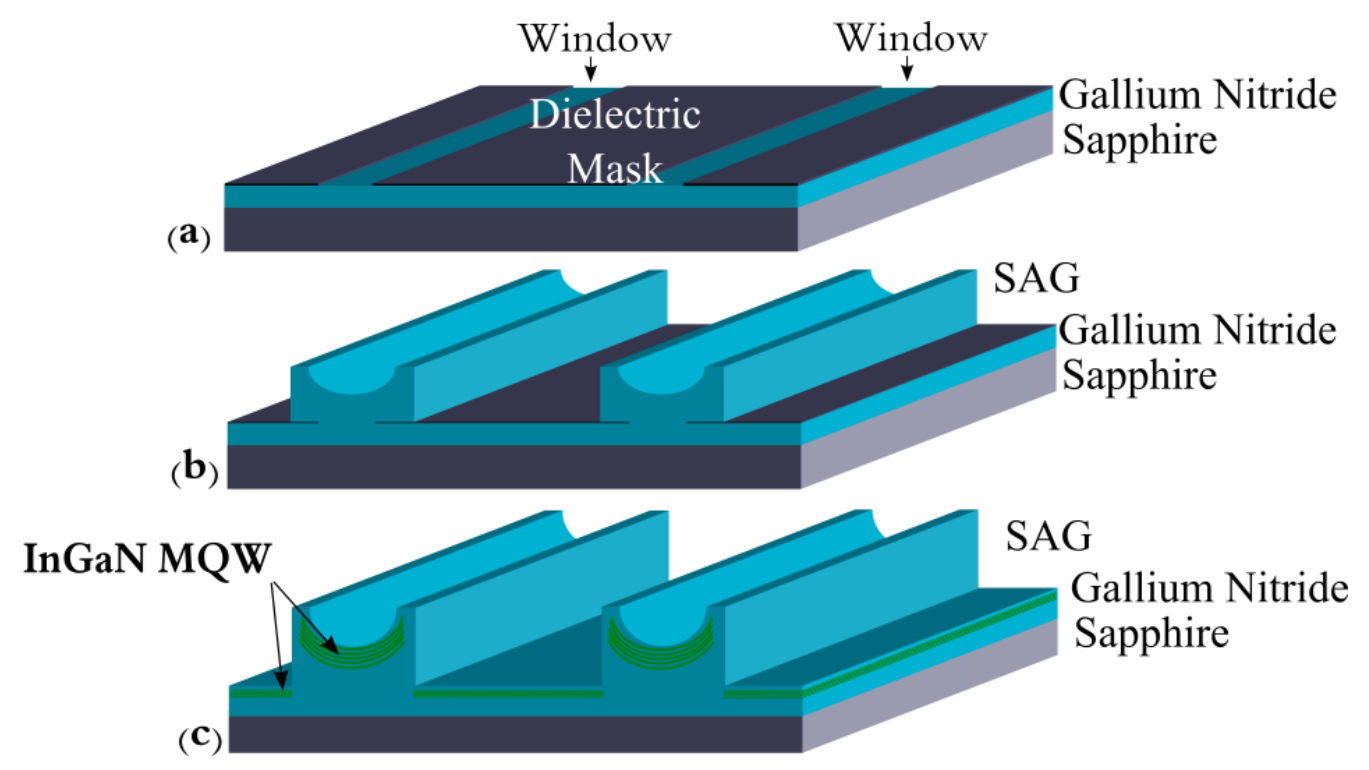

Figure 7-1 - (a) Dielectric mask used for SAG. Stripes oriented along $<1 \overline{1} 00>$ direction. (b) SAG of GaN showing curved cross section. (c) LED growth on SAG of GaN.

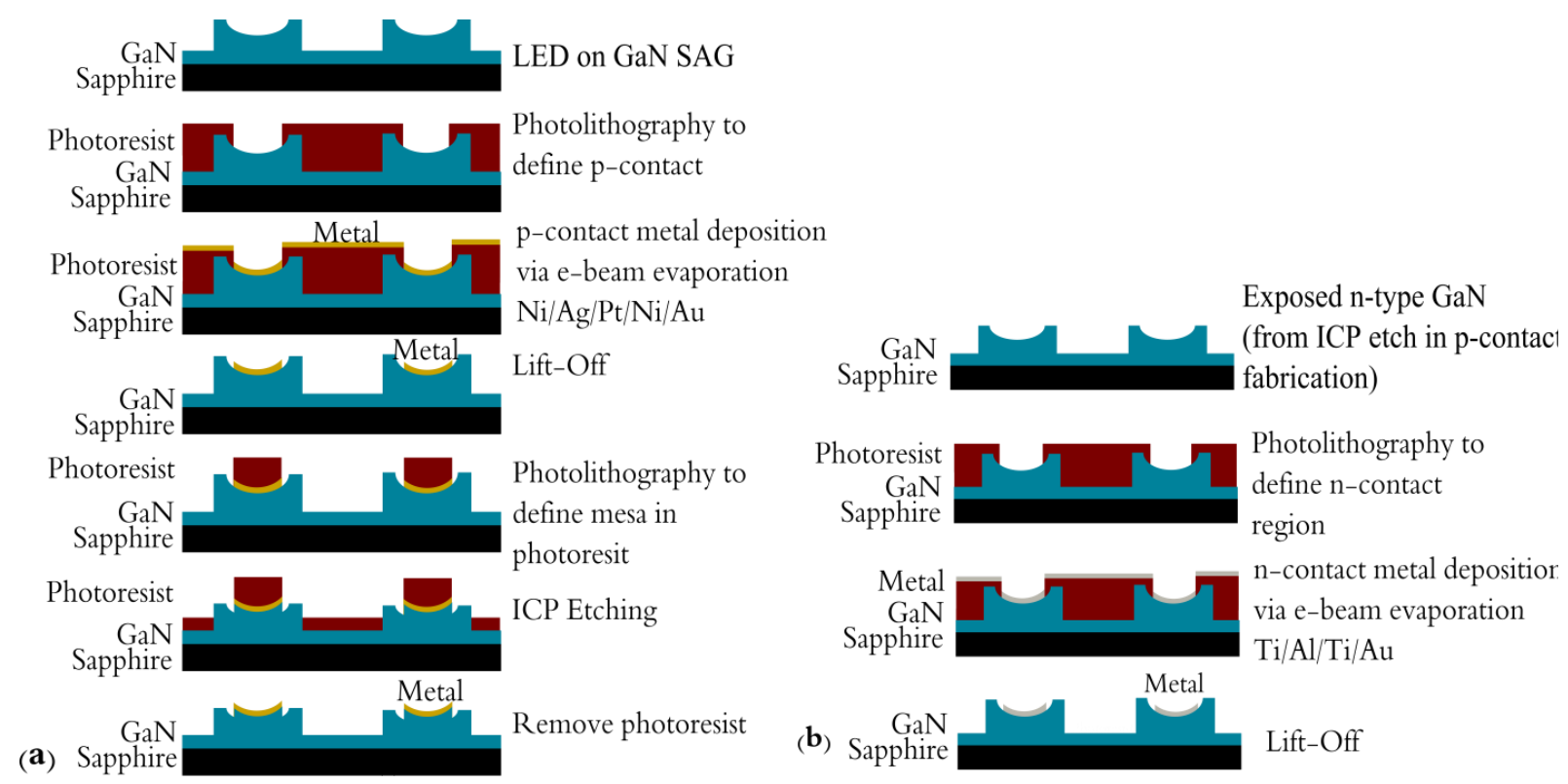

Figure 7-2 - (a) Process flow for p-type contact deposition and mesa etch. (b) Process flow for n-type contact deposition. 
shown in Figure 7-2a. The $p$-type contact consisted of $1 \mathrm{~nm} \mathrm{Ni} / 150 \mathrm{~nm} \mathrm{Ag} / 50 \mathrm{~nm} \mathrm{Pt} / 20 \mathrm{~nm}$ $\mathrm{Ni} / 50 \mathrm{~nm}$ Au deposited via e-beam evaporation in a Temescal BJD-2000 system. The LED mesa, approximately $100 \mu \mathrm{m}$ in diameter and $400 \mathrm{~nm}$ thick, was etched using a Trion MiniLock III ICP Reactive Ion Etcher. The ICP etch leaves several mesas along the length of the stripe and exposes the underlying $n$-type GaN. A circular $n$-type contact was then deposited beside the mesa using e-beam evaporation. This contact consisted of $2 \mathrm{~nm} \mathrm{Ti} / 100 \mathrm{~nm} \mathrm{Al} / 30 \mathrm{~nm} \mathrm{Ti} / 200 \mathrm{~nm}$ of Au. SEM images were taken to evaluate the curved structure and also the fabrication process. Standard I-V characteristics and EL measurements were taken and compared to the planar LEDs grown under the same conditions.

\subsection{Characterization}

Figure 7-3 contains the SEM cross-sectional images of the SAG of GaN grown on the silicon nitride mask. The cross sectional image shows a curved center with a flat terrace along the edge of the stripe. The mask exhibited selectivity however there was minor parasitic deposition observed in between the stripes, which is most likely due to the large width of the dielectric stripe used in this work. The V/III ratio has previously been reported to directly influence the formation of defects along the edge of the stripe ${ }^{144}$, however relatively smooth facets were observed using the growth conditions outlined above. Various V/III ratios and stripe dimensions were investigated to determine the effect on the growth of the curved structure. The V/III ratio and also the fill factor, defined as the window width/period, also affect the curvature
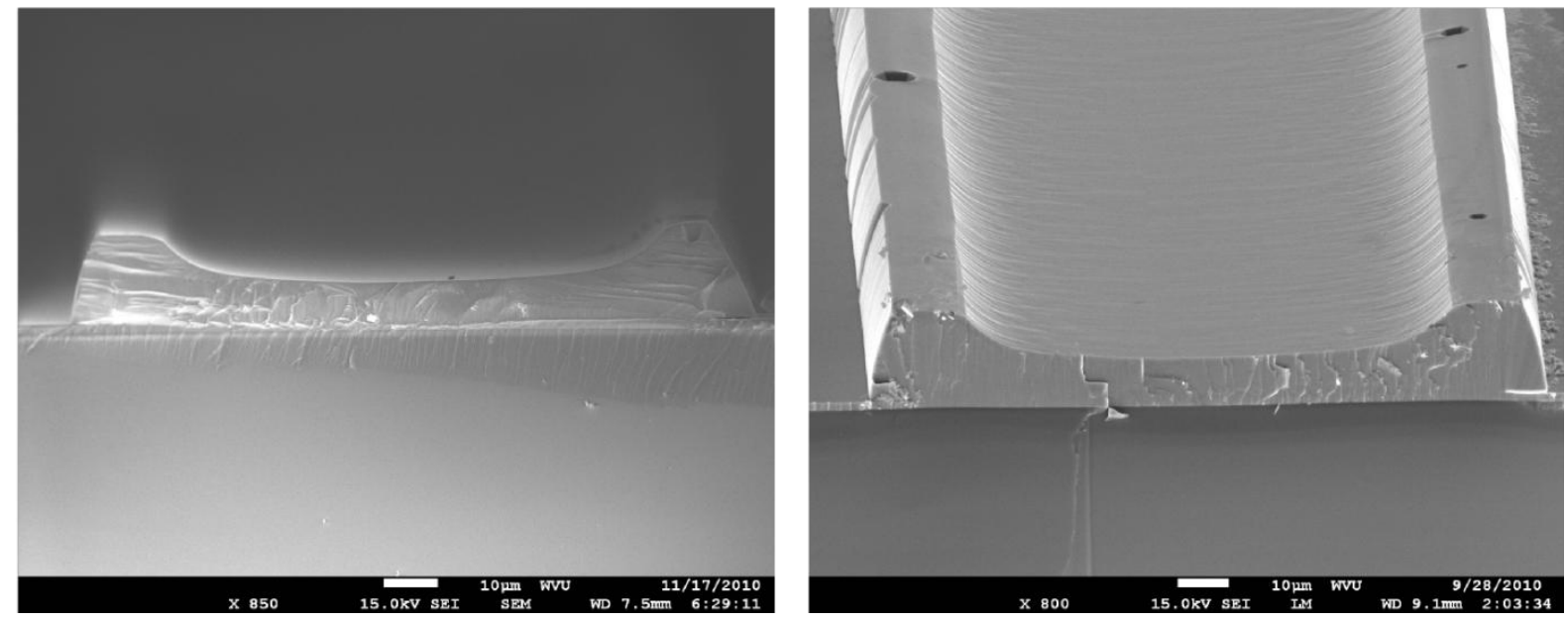

Figure 7-3 - Cross Sectional SEM of SAG of GaN using silicon nitride mask. 
of the SAG of GaN. The curvature was fitted as part of a larger circle and is shown in Figure 7-4. The mask material had an influence on the measured angle and is most likely due to a different sticking coefficient on the different dielectric masks leading to a different diffusion of the species to the growth site. Despite the mask material, the central angle decreased with increasing fill factor. In this work, the increase in fill factor corresponds to the increase in window width. The V/III ratio was also found to have a significant impact on the curvature of the SAG. Using these two factors, the angle of emission and also the planes of the SAG can be engineered.

Figure 7-5 contains an SEM image of the fabricated device. The circular $p$-type contact and mesa, as well as the circular $n$-type contact are shown.

Figure 7-6 contains the I-V characteristics as well as the electroluminescence of the LED on the curved GaN. The I-V measurements show high resistance most likely due to the large distances between the $n$-type and $p$-type contacts used in this work. The EL spectrum contains both the emission from the curved LED as well as from a planar device grown under the same conditions. The EL measurement from the curved LEDs exhibit a slightly broader emission spectra when compared to the equivalent planar structures, however it is centered at a similar wavelength. Since the dielectric mask was removed via wet etching prior to the LED growth, there is no additional contribution of gas phase diffusion of species from the mask to the growth site as is commonly observed in the SAG of $\operatorname{In}_{x} \mathrm{Ga}_{1-x} \mathrm{~N}$ MQWs ${ }^{12}$. The broader emission may therefore be due to a difference in growth rate and/or indium incorporation along the different facets that compose the curved structure. It has been well established that in III-Nitride materials, the different planes have varying degrees of stability and can influence the indium incorporation and quantum well thickness ${ }^{13}$. The resources to accurately measure these features on the curved structure are not currently available. Future work will focus on optimizing the growth conditions for individual layers on the curved surface, at which time TEM will provide more valuable information. 


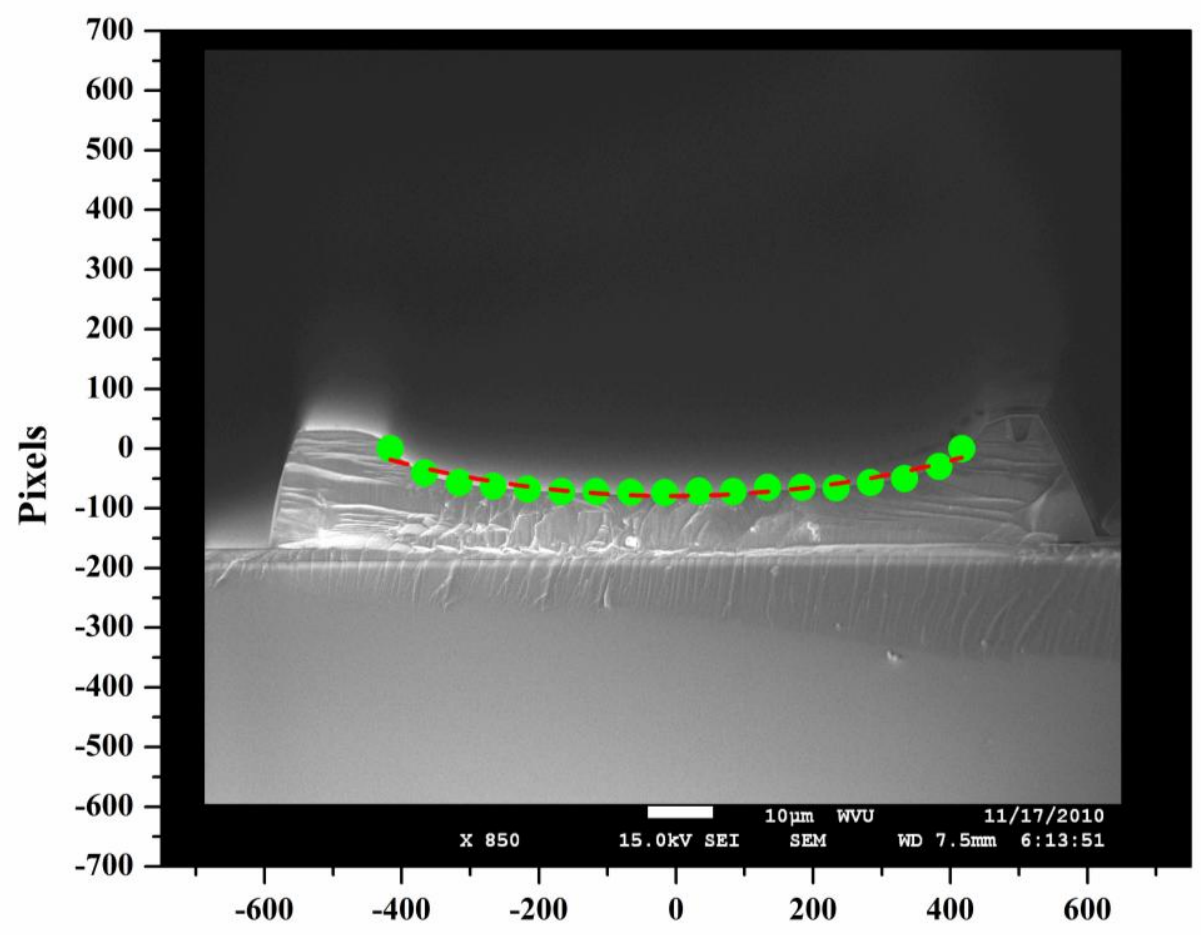

(a)

Pixels

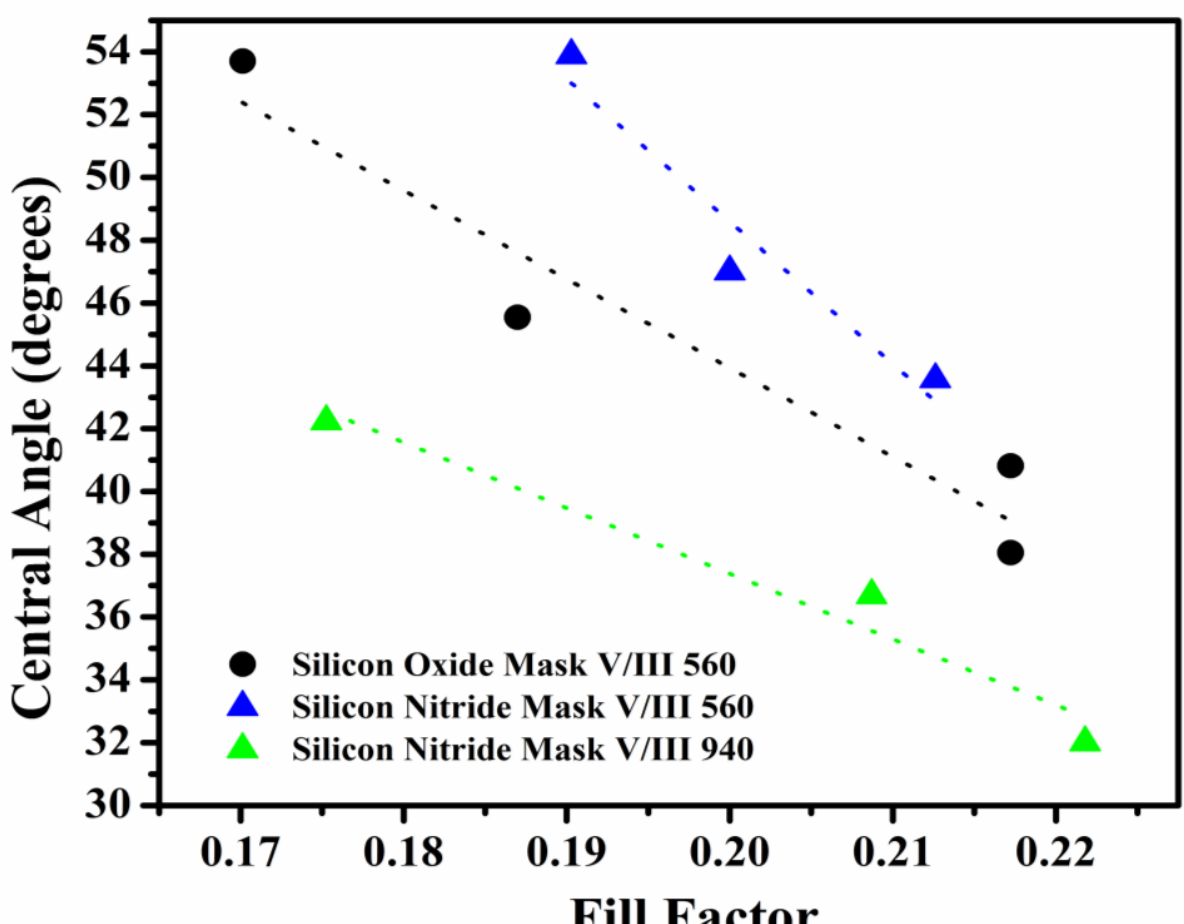

(b)

Figure 7-4 - (a) Fitting points along SAG structure as circle. (b) Relationship between the central angle of the fitted circle to the fill factor and V/III ratio. 


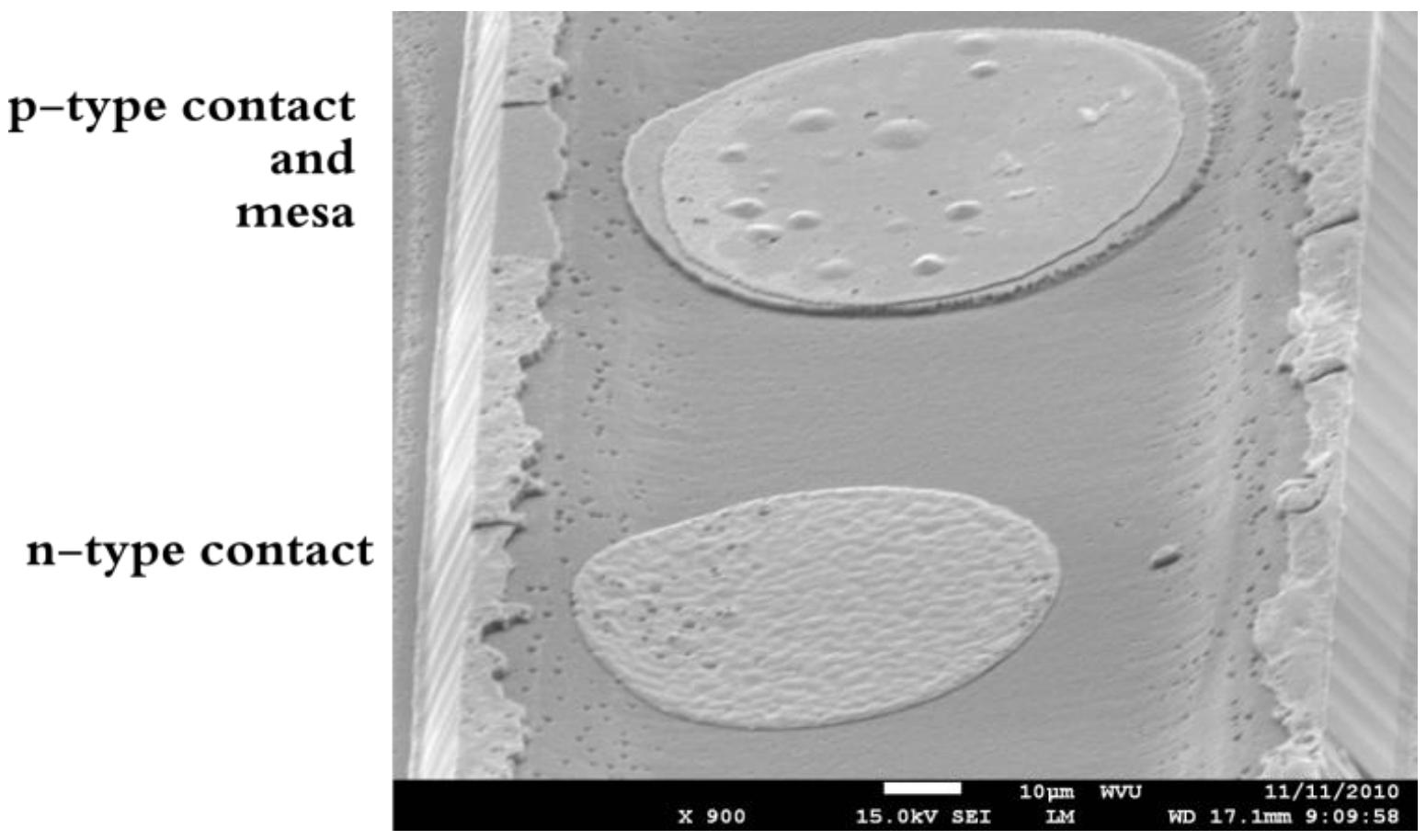

Figure 7-5 - SEM image of fabricated LED on curved GaN SAG.

In summary, this work has demonstrated the growth and fabrication of III-Nitride based LEDs on curved GaN structures fabricated through SAG via MOVPE. A striped pattern along the $<1 \overline{1} 00>\mathrm{GaN}$ direction with window widths ranging from $80-110 \mu \mathrm{m}$ was used for the SAG. Silicon nitride and silicon oxide was used as the mask materials and exhibited good selectivity under the growth conditions employed, however minor parasitic deposition in the mask area was observed. Simple five period $\operatorname{In}_{x} \mathrm{Ga}_{1-x} \mathrm{~N} / \mathrm{GaN}$ MQW based LED structures were grown on the curved GaN stripes and devices were fabricated along the length of the stripe using standard fabrication procedures. The emission from the curved LED is slightly broader when compared to a planar device grown under the same conditions and may be due to the different growth kinetics along the different facets that compose the curved surface. This work demonstrates the ability to fabricate LEDs on curved surfaces and has potential applications in engineering the emission pattern and spectrum of nitride based LEDs by utilizing growth kinetics. 


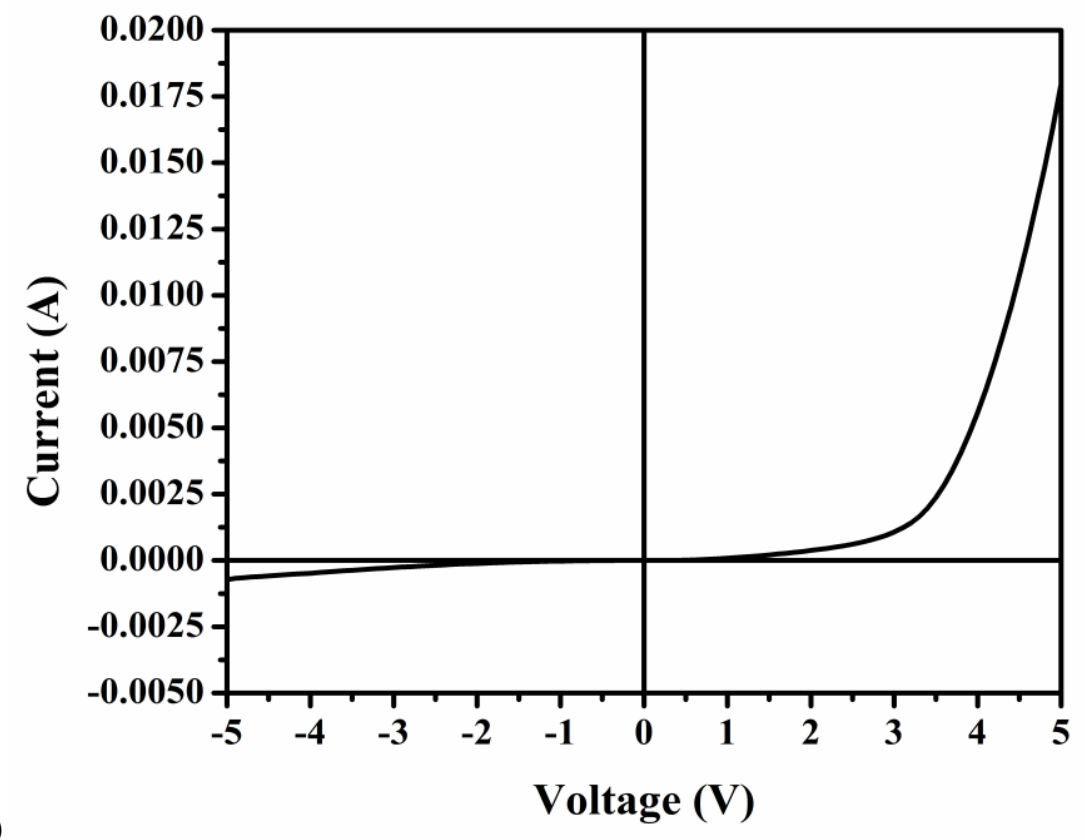

(a)

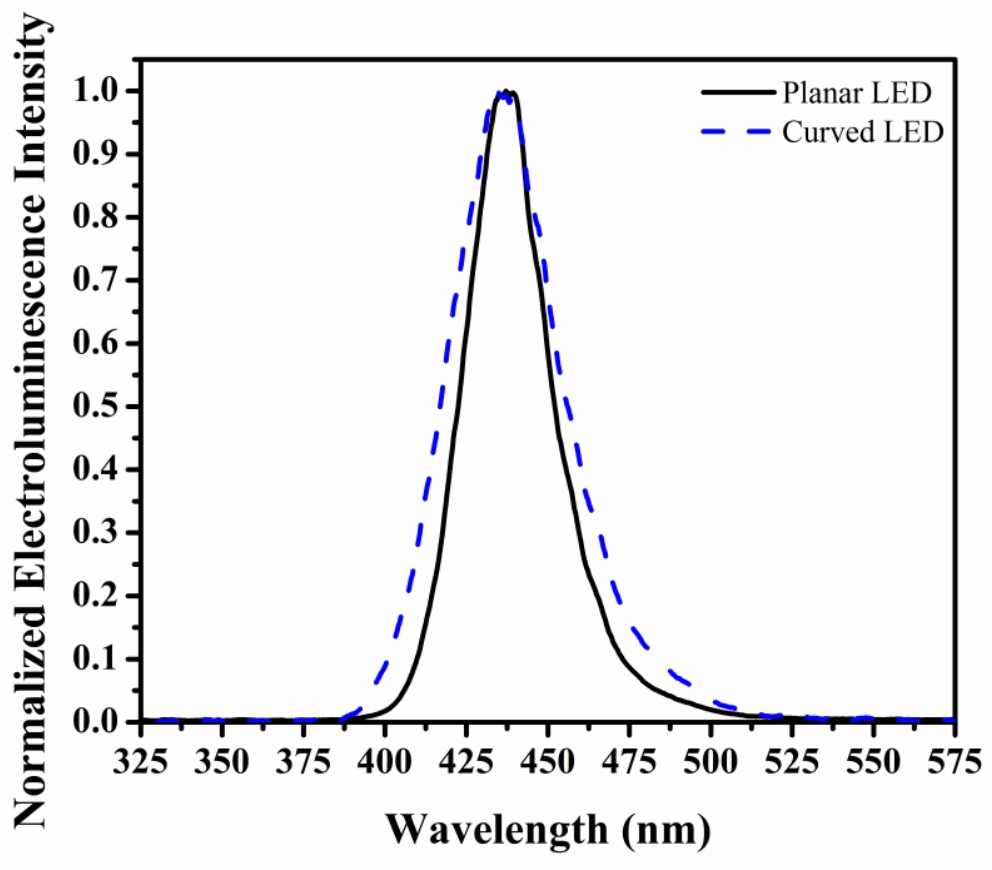

Figure 7-6 - (a) Current-Voltage characteristics of curved LED and (b) electroluminescence spectra of curved and planar LED. 


\section{CHA P T E R 8 - S E L E C T I VE A R E A G R O W TH A N D \\ E P I T A I X I A L L A T E RA L O V E R G R O W T H}

\subsection{Introduction}

As described previously, the Epitaxial Lateral Overgrowth (ELOG) is one common technique used to reduce the dislocation density reaching the surface of the epilayer. In this work, the ELOG process has been established using the MOVPE tool at WVU. As the ELOG process relies heavily on the Selective Area Growth (SAG) of GaN, it has been investigated both with and without rotation ${ }^{145,146,147}$ in order to develop a deeper understanding of the mechanisms governing this process and for three dimensional device geometries.

\subsection{Selective Area Growth}

During SAG of GaN, a dielectric masked is deposited on a GaN epilayer. The mask region consists of features on the order of 5-10 $\mu \mathrm{m}$ with exposed regions of the underlying GaN. When growth is conducted under certain conditions on this patterned substrate, the precursors will diffuse over the dielectric mask to the exposed GaN at which point they will be incorporated into the growth. Therefore, unique geometries can be achieved through the mask design and also through the growth conditions. In this work, a striped silicon dioxide mask was used as shown in Figure 8-1. An approximately $1.5 \mu \mathrm{m}$ thick GaN film was grown on a two inch diameter $c$-plane sapphire wafer via MOVPE using a two step process involving a $\sim 30 \mathrm{~nm}$ AlN nucleation layer followed by

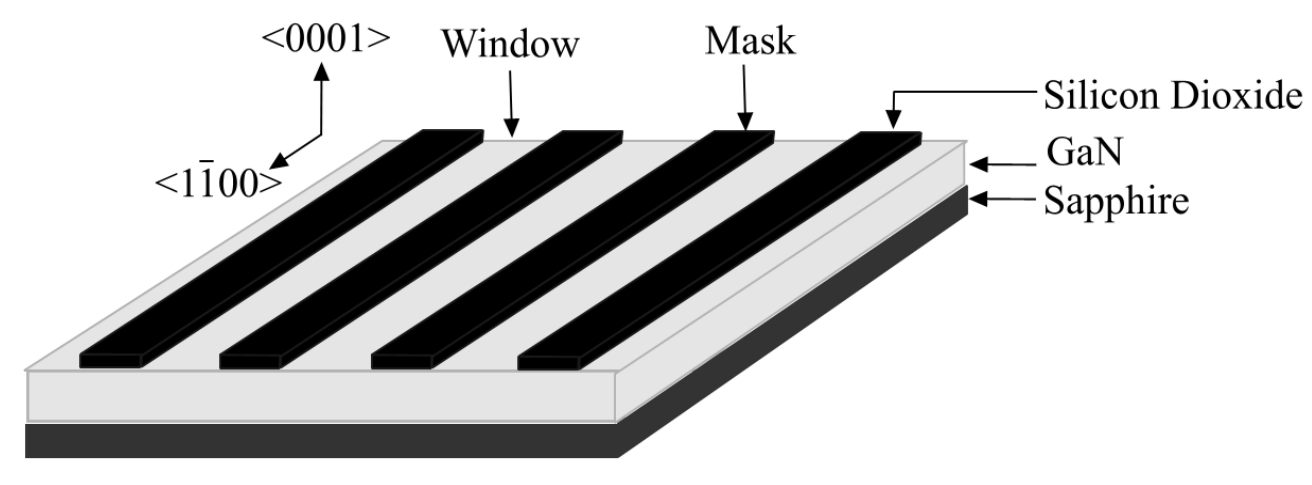

a

Figure 8-1 - (a) Striped pattern along GaN $<1 \overline{100}>$ direction 
a high temperature $\mathrm{GaN}$ main layer. $\mathrm{TMGa}$ and $\mathrm{NH}_{3}$ were used as the source gasses while hydrogen and nitrogen were used as the carrier gases. Next, a $130 \mathrm{~nm}$ layer of silicon dioxide was deposited via Plasma Enhanced Chemical Vapor Deposition (PECVD) by an Oxford Plasma Lab 80 Plus system. Standard photolithography and wet etching techniques were used to create a striped pattern in the silicon dioxide layer along the $\mathrm{GaN}<1100>$ direction. The SAG of GaN was performed at different temperatures and V/III ratios. The window opening and the stripe width for these samples were both $5 \mu \mathrm{m}$. Figure 8-2 shows cross sectional Scanning Electron Microscopy (SEM) images of SAG illustrating the control over cross sectional geometry that can be achieved by tailoring the growth conditions. Consistent with literature reports, growth at a low temperature (image a) resulted in slow growth rate and poor surface morphology. Increasing the

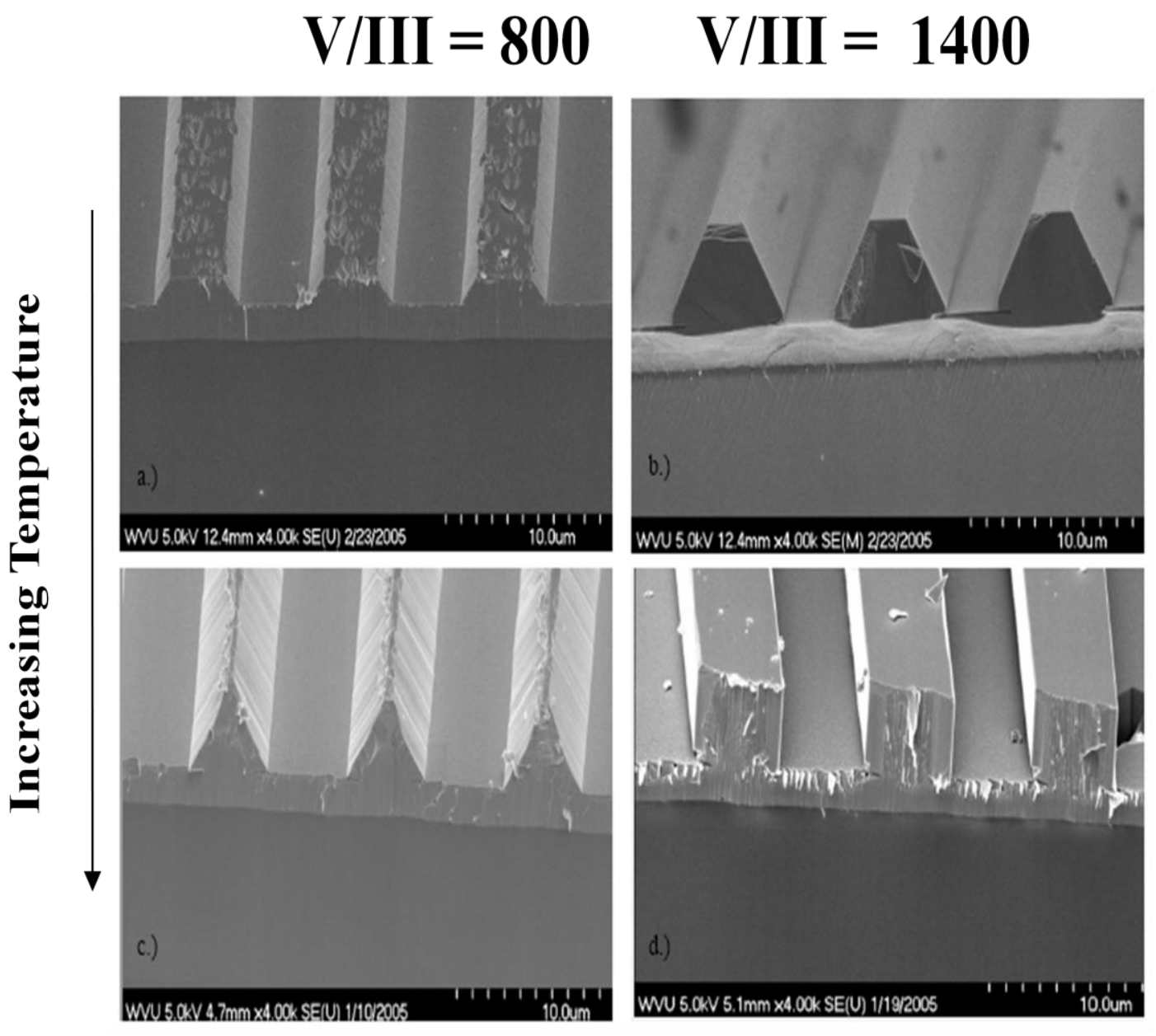

Figure 8-2 - Scanning Electron Microscopy images of GaN SAG as a function of temperature and V/III ratio. a.) Step-one at $960^{\circ} \mathrm{C}$ for 40 minutes, b.) Step-two at $1110^{\circ} \mathrm{C}$ for 90 minutes. c.) Step-one at $1050^{\circ} \mathrm{C}$ for 40 minutes, d.) Step-two ELOG at $1200^{\circ} \mathrm{C}$ for 90 minutes. 
temperature as shown in image c results in the triangular cross section suitable for ELOG. In this figure, the second column (images b and d) are the results after re-growth on samples shown in the first column (images $a$ and $c$ ) at a higher temperature and V/III ratio. Images $b$ and $d$ show the evolution of the faster lateral growth rate with increasing temperature and V/III ratio. Figure 8-3 shows the relationship between temperature and the ratio of lateral to vertical growth rate. As the temperature is increased, there is an increase in growth rate up to the point that species desorption from the surface results in a decrease in growth rate. These unique geometries have potential applications in device design. Additionally, since the facets of the three-dimensional planes are semi or non-polar planes, they are often pursued for device fabrication.

\subsection{Epitaxial Lateral Overgrowth}

As discussed previously, the triangular cross section is critical for the two step ELOG process in order to induce the dislocation bending. Based on the results of the SAG of GaN discussed, conditions were identified to fabrication structures with the triangular cross section as shown in Figure 8-2c. In order to consistently obtain triangular structures, the dielectric mask used was modified to have a $3 \mu \mathrm{m}$ window width and a $7 \mu \mathrm{m}$ stripe width. There are several different approaches that have been reported for the second step of the ELOG process in order to induce the lateral overgrowth. In this work, a high growth temperature was used. Temperatures 55-100 degrees higher than those used in the first step of the process were targeted. Figure 8-4 shows the cross sectional SEM images of the second step of the ELOG process conducted as different temperatures. Each sample shown was grown for the same amount of time. It should be noted that the temperatures reported are the nominal MOVPE temperatures used during growth. The system temperature was recalibrated between the time the SEM images in Figure 8-2 and Figure 8-4 were taken and is the cause for the apparent discrepancy in temperatures. After calibration, the temperature used to achieve the triangular cross section was approximately $960{ }^{\circ} \mathrm{C}$. As can be seen in Figure 8-4, the performing the second ELOG step at $1015{ }^{\circ} \mathrm{C}$ was not sufficient to enhance lateral growth enough to achieve full coalescence. Increasing the temperature to $1040{ }^{\circ} \mathrm{C}$ as shown in image $b$ resulted in full coalescence and a smooth surface morphology. Increasing the temperature even further, as shown in image c, results in coalescence however, the process was not as uniform over the entire sample compared to that achieved at $1040^{\circ} \mathrm{C}$. Therefore, the growth 


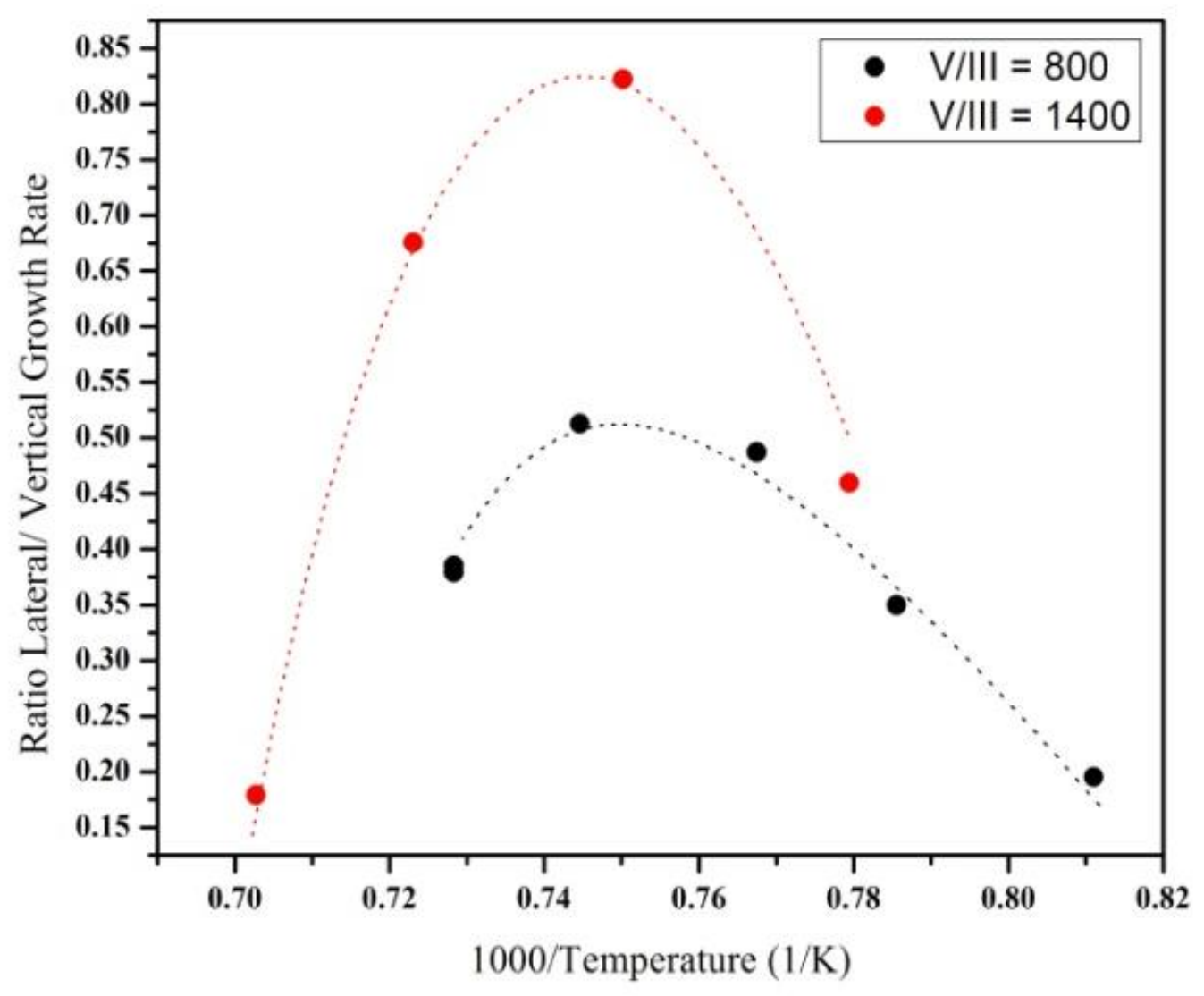

Figure 8-3 - Ratio of lateral to vertical growth rate as a function of growth temperature.
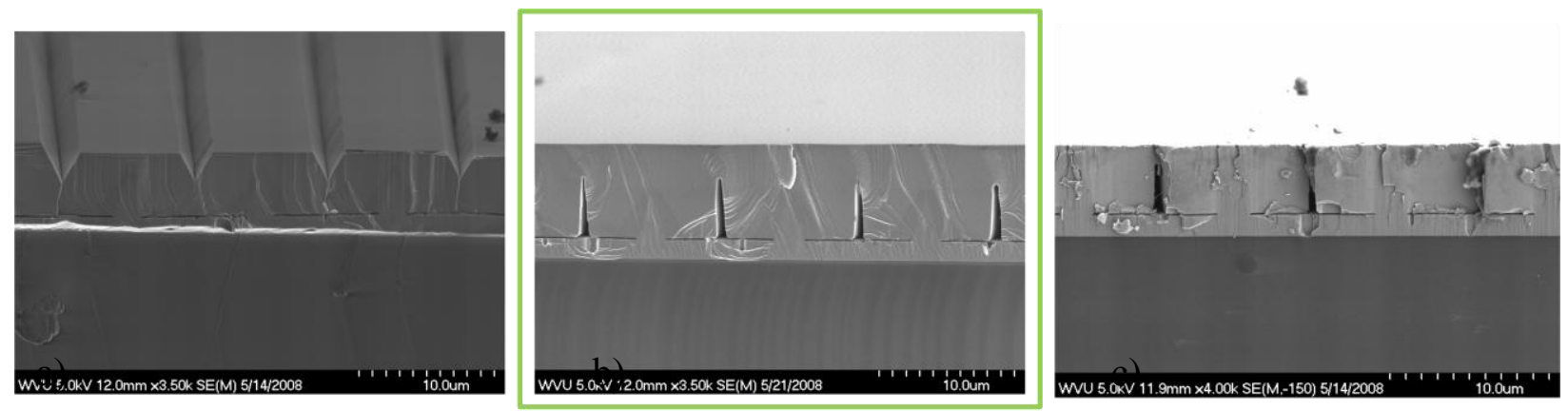

Increasing Temperature

Figure 8-4 - Second step of ELOG at different growth temperatures. a) $1015^{\circ} \mathrm{C}$ b) $1040^{\circ} \mathrm{C}$ and c) $1065^{\circ} \mathrm{C}$ 


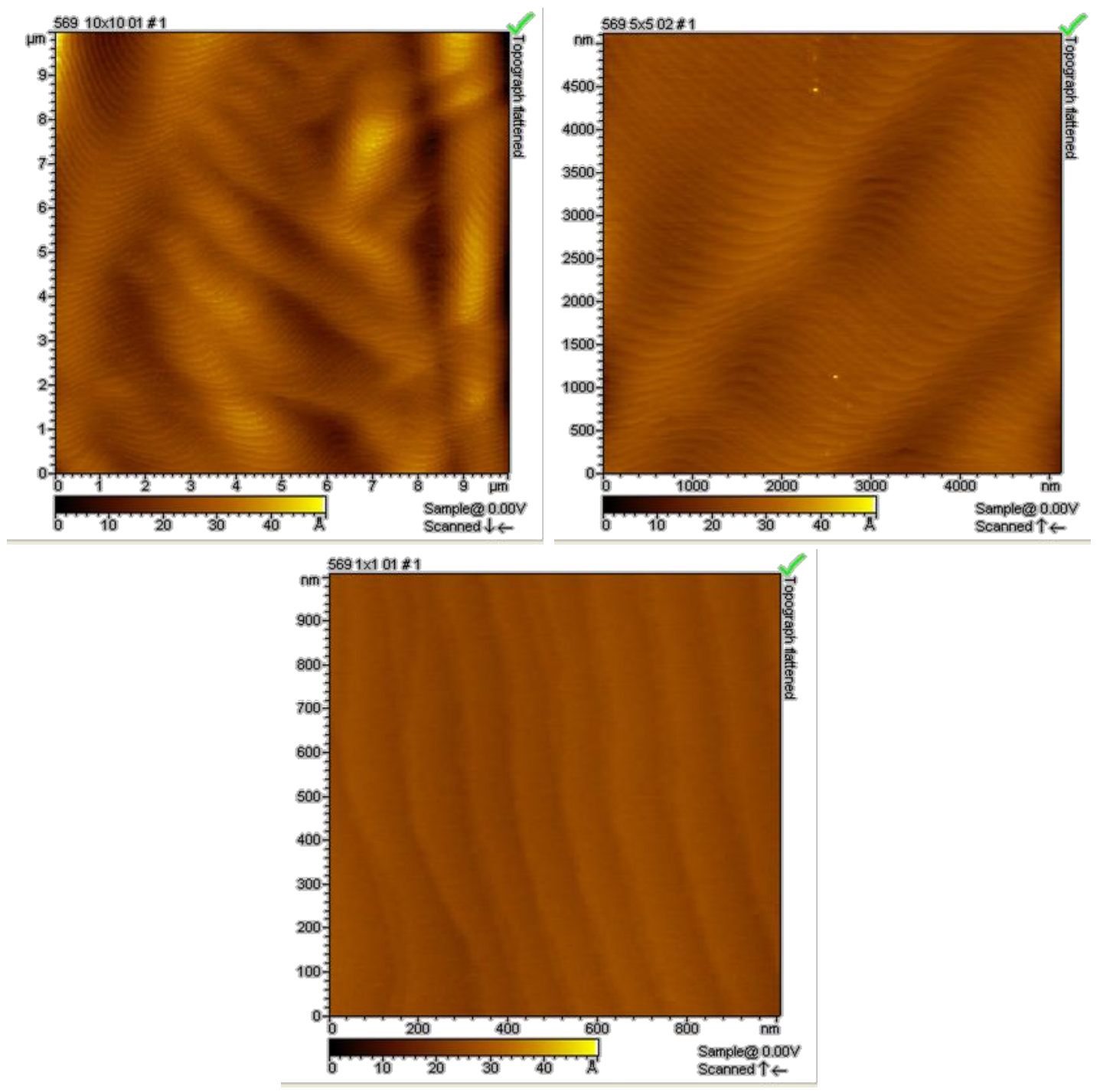

Figure 8-5 - AFM images of the surface morphology of two step ELOG process.

temperature used in image $b$ was chosen as the ideal temperature. Figure 8-5 contains two AFM images of the surface of the two step ELOG process. As can be seen in $1 \mu \mathrm{m} \times 1 \mu \mathrm{m}$ scan, the surface shows the atomic steps as described previously. The RMS roughness for the $5 \mu \mathrm{m} \times 5 \mu \mathrm{m}$ scan is $0.77 \mathrm{~nm}$ indicating a very smooth surface.

\subsection{Selective Area Growth with No Rotation}

In order to investigate the effects of gas flow on the SAG of GaN, two similarly sized pieces patterned with the $\mathrm{SiO}_{2}$ mask as discussed in the previous section, were placed side by side in a line normal to the incoming gas flow in the reactor for re-growth. One sample was 
placed with the striped pattern oriented parallel to the incoming gas flow and the other with the striped pattern oriented normal to the incoming gas flow as shown in Figure 8-6. In roughly half of the growths, the samples with the striped pattern oriented parallel to the incoming gas flow were located on the left hand side of the incoming gas, as shown in Figure 8-6. In the other growths, the sample with the striped pattern oriented parallel to the incoming gas flow was located on the right hand side. This configuration was used in order to simultaneously fit two samples on the two inch susceptor and ensure similar growth conditions by eliminating the effects of uneven gas flow and substrate heating. The samples were grown with no rotation at $1115^{\circ} \mathrm{C}, 200 \mathrm{mbar}$, and a V/III ratio around 1400 . The ammonia and the carrier gas flow rates were around $1.2 \mathrm{slm}$ and $4.0 \mathrm{slm}$, respectively. The cross sections of the resulting three dimensional structures were observed using a Hitachi S4700 Scanning Electron Microscope (SEM) and conventional image processing techniques were used to determine the size of the structures. For practical reasons, one piece per growth per orientation was evaluated and each piece characterized was about one quarter of the size of the grown sample. In order to eliminate experimental artifacts in the analysis of the results, such as temperature non-uniformity, images and measurements were taken from areas of the two samples that were closest during growth. Any variations in the sample are summarized by the error bars shown in each figure.

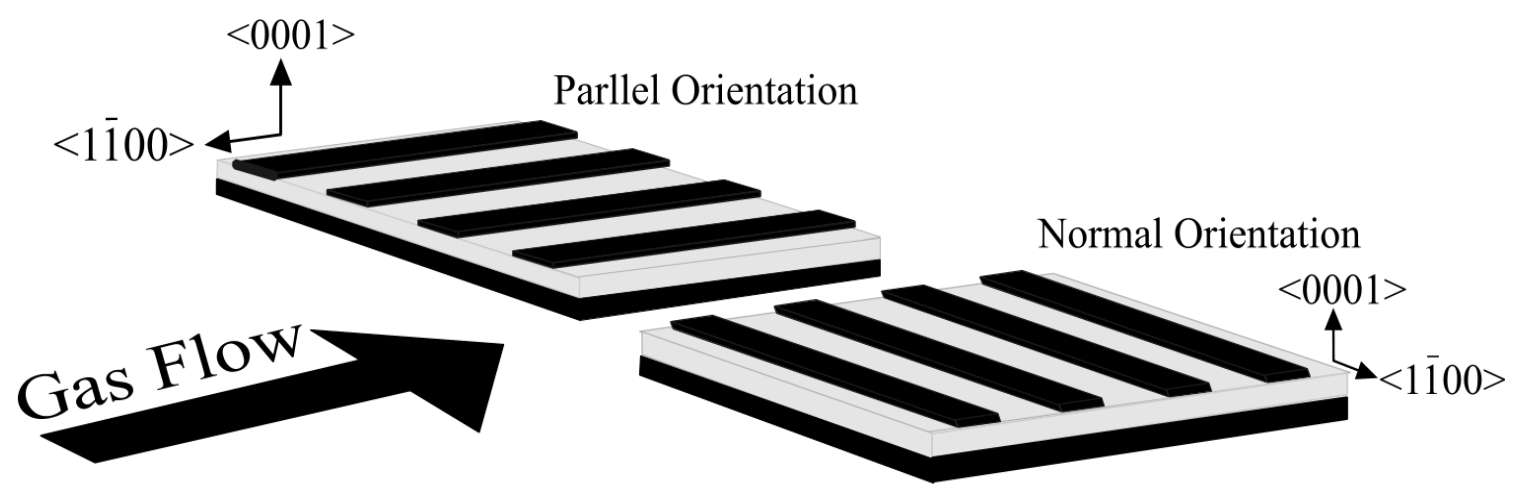

b

Figure 8-6-(a) Striped pattern along GaN <1100> direction(b) Samples oriented parallel and normal to the incoming gas flow during selective area growth of GaN. 
Occasionally, pieces from other parts of the sample, both off center and further downstream were evaluated. Based on SEM images, the growth results were consistent across the samples and fall within the shown error bars. Figure 8-7 and Figure 8-8 show the SEM images of the cross section for the samples with stripes oriented parallel and normal to the incoming gas flow. After 30 minutes of growth both orientations exhibited similar growth modes resulting in the same trapezoidal structure with the (0001), $\{11 \overline{2} 2\}$, and $\{11 \overline{2} 0\}$ facets exposed shown in Figure 8-7. Therefore, all subsequent growths were performed on this base structure and the growth rates were measured as an increase in size from this base structure. It should be noted that no asymmetry in the structures was observed for either orientation as might be expected. Throughout later growth stages, both samples maintained a similar trapezoidal cross section with (0001), and $\{11 \overline{2} n\}$ facets $(n \approx 1.7-2.2)$ exposed. The samples oriented normal to the flow experienced larger growth rates, as is indicated in Figure 8-9 and Figure 8-10.

Figure 8-10 illustrates the height as a function of growth time. Clearly, the normally oriented sample experiences a larger vertical growth rate when compared to the parallel oriented

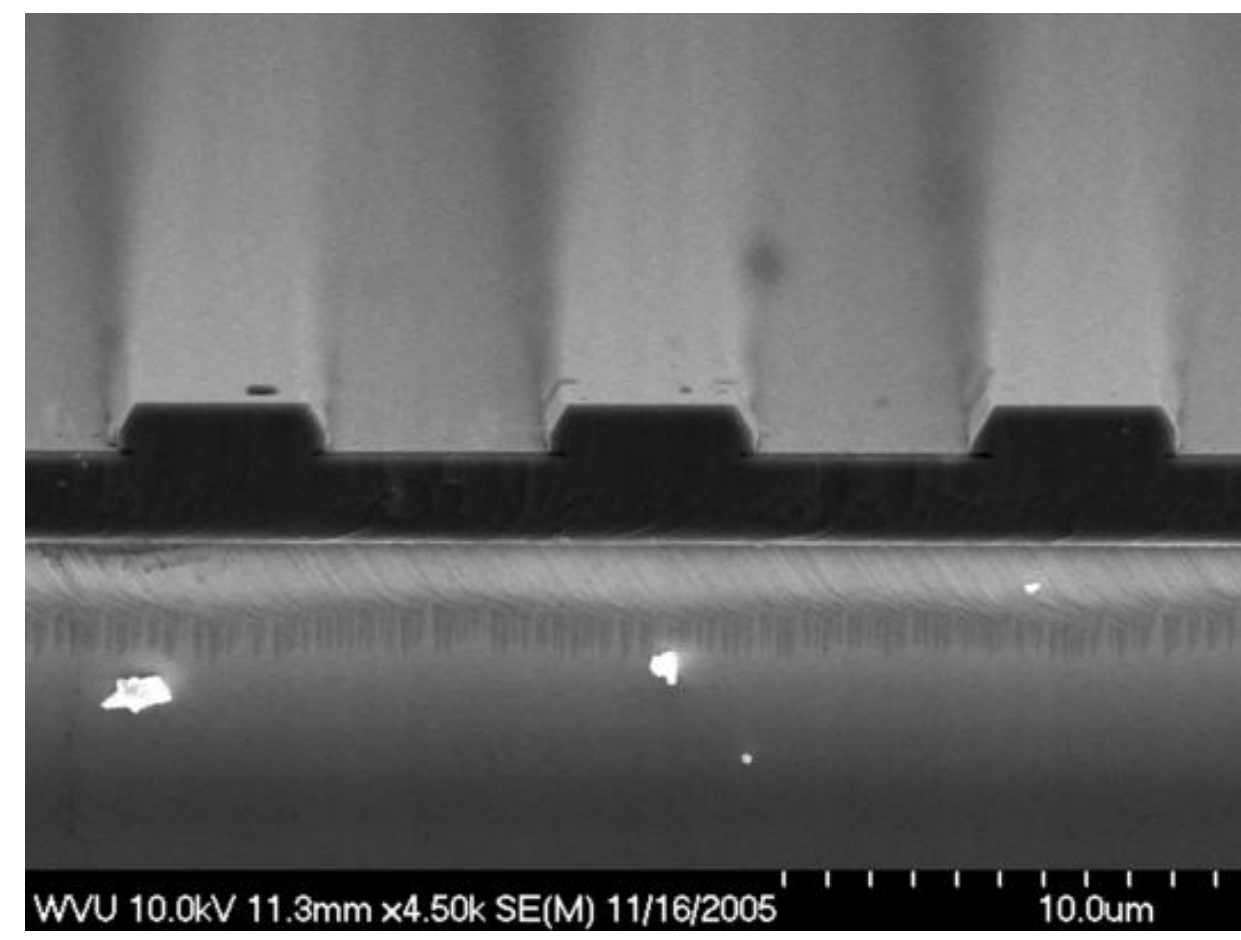

Figure 8-7 - SEM image of SAG base structure. Structure was identical for samples with stripes oriented normal or parallel to incoming gas flow. 

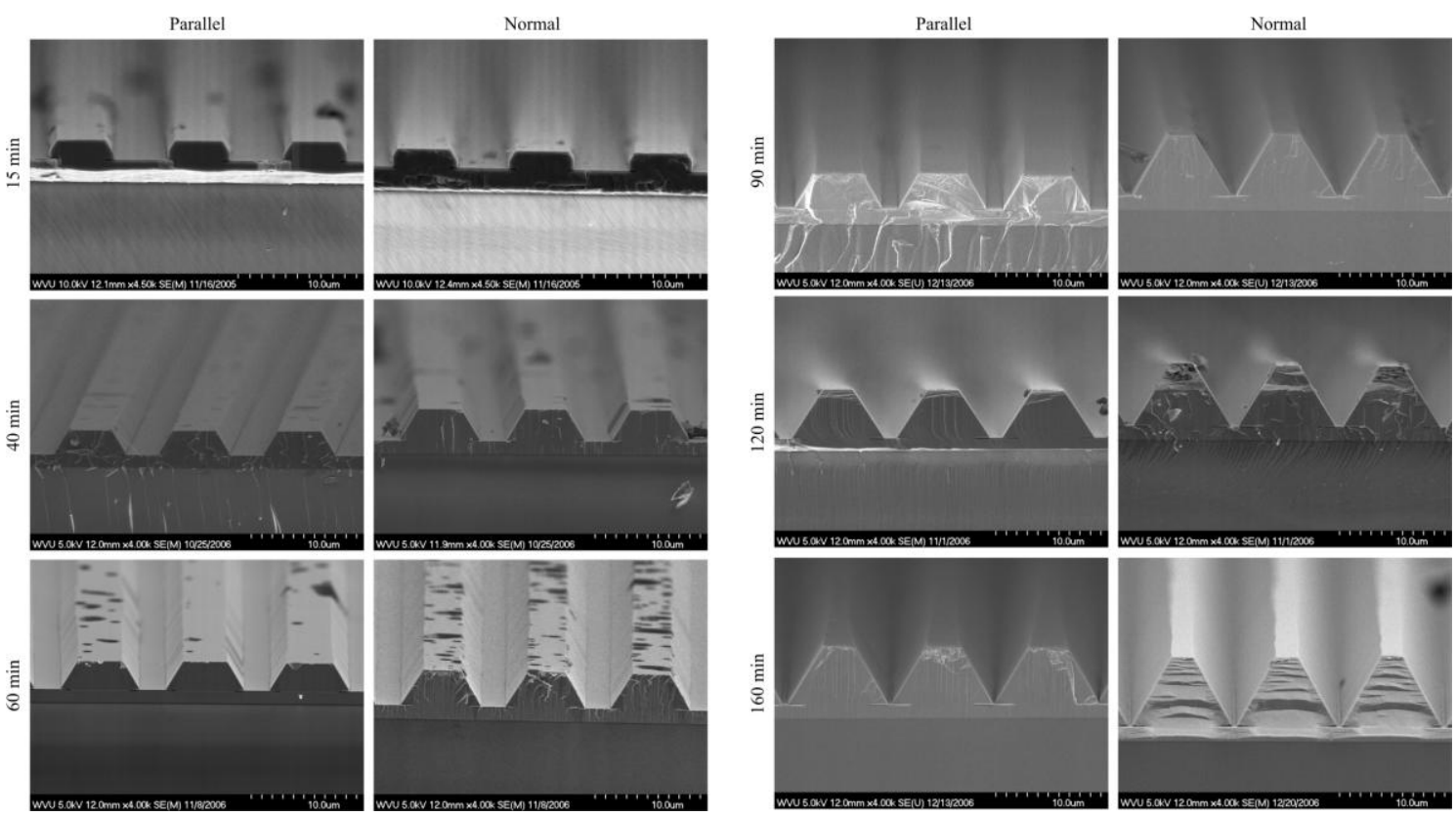

Figure 8-8 - SEM images of selective area growth of GaN with $<1100>$ striped pattern oriented parallel and normal to the incoming gas flow.

sample. Figure 8-9 illustrates the cross sectional area of the three dimensional structures per pattern period. Once again, the normally oriented sample is displaying an enhanced cross sectional growth rate. In both Figure 8-9 and Figure 8-10, the line is a linear fit using the error bars as weight. Also shown in Figure 8-9 and Figure 8-10 is the height and cross section of the structures when SAG is carried out for 120 minutes while rotating the sample. The height of the rotating sample is similar to that of the normal oriented sample, while the cross section is similar to the parallel oriented sample. Since the growth conditions are identical for both orientations, the increased cross sectional growth rate of the normal oriented sample indicates that more species are being incorporated into the growth. Both orientations maintain exposed (0001), and $\{11 \overline{2} n\}$ facets $(n \approx 1.7-2.2)$ indicating that there is no difference in surface growth kinetics between the samples. In MOVPE, it is assumed that the gallium species sticking coefficient is unity on the GaN surface ${ }^{36}$ and there is efficient lateral transport across the oxide mask allowing all reactants reaching the surface to be incorporated into the SAG growth ${ }^{148,149}$. Therefore, it can be concluded that there is a difference in mass transport between the normal and parallel oriented sample to account for the difference in growth rate. The normal orientation experienced 
enhanced growth rate because more species were diffusing to the growth site. The size of the structures is negligible when compared to the boundary layer thickness, which is proportional to the square root of the kinematic viscosity of the gas and the distance from the edge of the susceptor and inversely proportional to the square root of the free stream gas velocity ${ }^{26,150}$. Therefore, it can be assumed that the height of the structure does not have a significant impact on the boundary layer, which is consistent with the fact that both orientations maintain constant growth rates even as the structures grow taller. These findings are contrary to the results obtained when the effect of gas flow was investigated on cubic $\mathrm{GaN}^{151}$. Cubic GaN was reported to demonstrate only a slight dependence of the cross sectional geometry and growth rates on the orientation of the pattern with respect to the incoming gas flow. Future work is needed to further explain the mechanism responsible for dependence of growth rates on the orientation of the striped pattern with respect to the incoming gas flow.

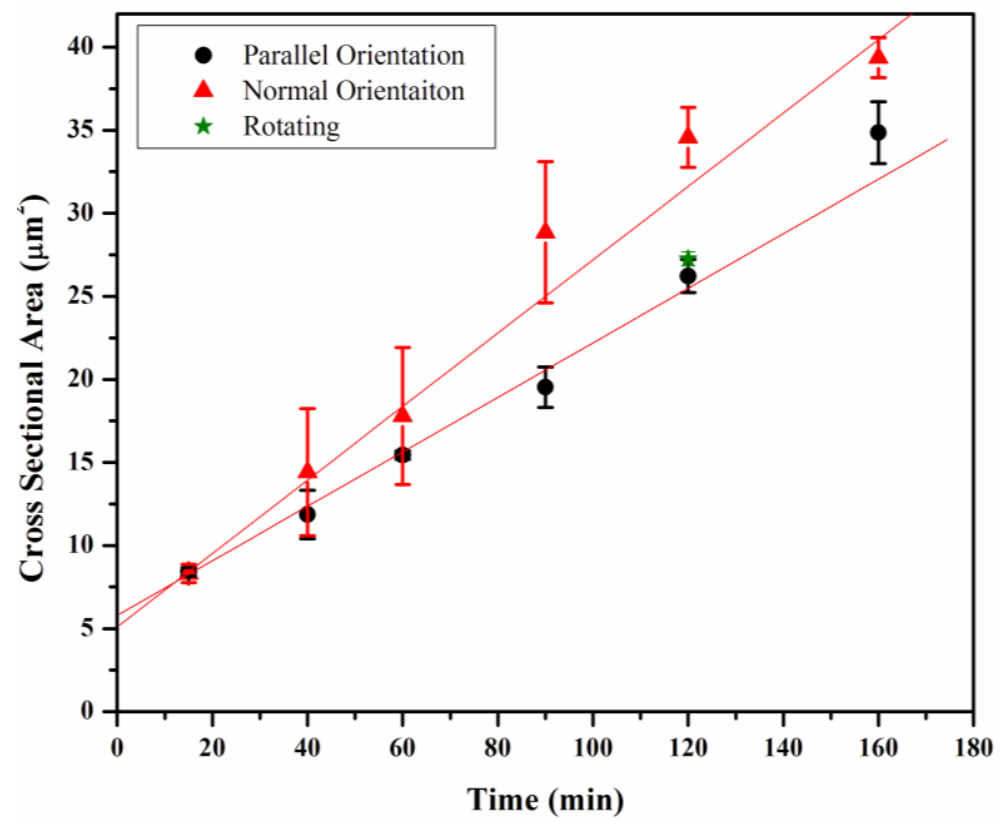

Figure 8-9-Cross section as a function of time for normal and parallel oriented samples. The lines are a linear fit of the data using the error bars as weight. The y-axis intercept corresponds to the cross sectional area of the base structure. 


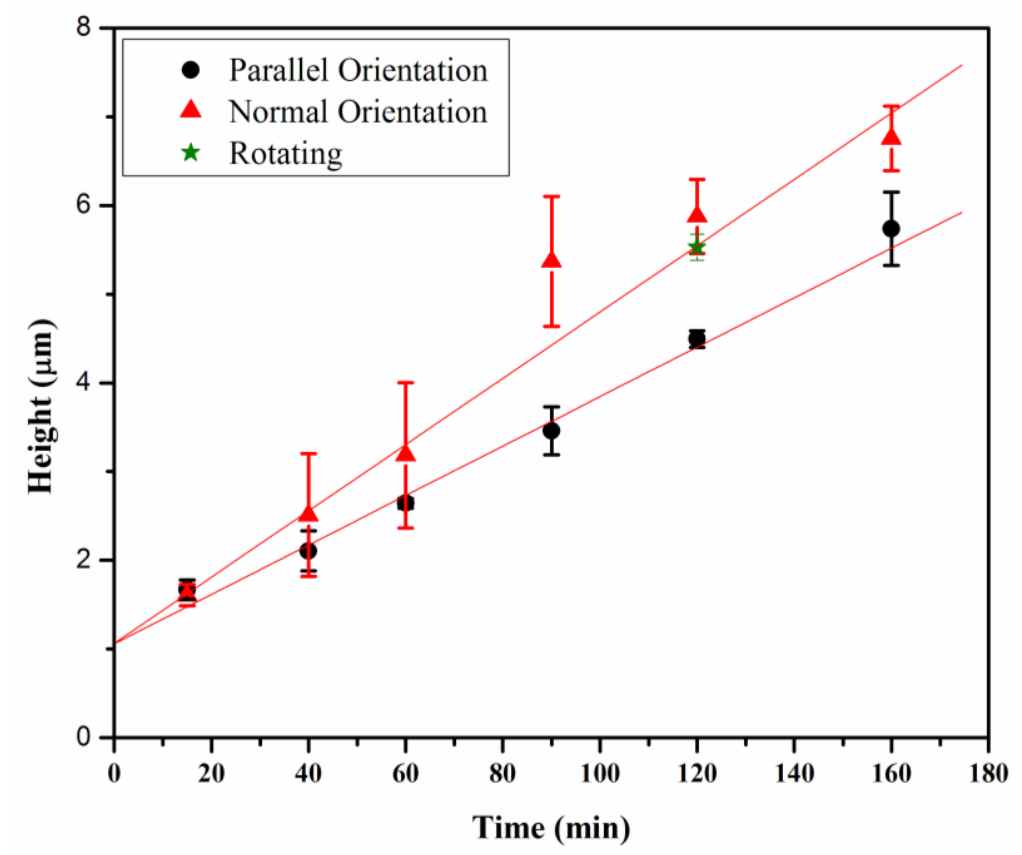

Figure 8-10 - Height as a function of growth time for parallel and normal oriented samples. The lines are a linear fit of the data using the error bars as weight. The y-axis intercept corresponds to the height of the base structure. 


\section{CHA P T ER 9 - A L U M I NUM NITRIDE SUSPENDED S T R U C T U R E S}

\subsection{Introduction}

AIN is often integrated with MEMS devices utilizing suspended structures, such as mirco-bridges or cantilevers, for use as resonators, transducers, or RF switches ${ }^{152,153}$. In general, the fabrication of suspended structures is complicated by the selection and removal of the sacrificial layer with respect to the functional material and substrate. Much attention has been focused on sputtered AlN due to its low cost and deposition temperature. Since it is widely known that the crystalline quality of AlN strongly affects the etch rate in certain acids ${ }^{154,155,156}$ and sputtered films are polycrystalline in nature, they often require a protective and supportive layer such as Silicon Nitride $\left(\mathrm{Si}_{3} \mathrm{~N}_{4}\right)$ or polysilicon ${ }^{157,158}$ in order to protect the functional material during the removal of the sacrificial layer and also provide the suspended framework.

More recently, growth techniques, such as MOVPE ${ }^{159}$ and $\mathrm{MBE}^{154}$, have been employed for the fabrication of nitride based suspended structures. In these techniques, the sacrificial layer must serve as a pseudo-substrate for subsequent growth, but must also be able to be selectively removed. Most techniques have specifically targeted the fabrication of Gallium Nitride (GaN) based MEMS and NEMS structures by employing polycrystalline ${ }^{154}$, epitaxial III-Nitride alloy ${ }^{160}$, and recently nanocrystalline ${ }^{159}$ films as sacrificial layers. The fabrication techniques are relatively complex due to the high dependency on the material properties of the functional and sacrificial layers (e.g. bandgap, polarity, crystallinity, etc). Furthermore, the sapphire substrates used in these techniques limits the integration with current silicon MEMS technology.

This work presents a growth based fabrication technique for achieving unsupported suspended AlN structures grown via MOVPE using Silicon Oxide $\left(\mathrm{SiO}_{\mathrm{x}}\right)$ sacrificial layers ${ }^{161,162,163,164,165}$. AlN grown via MOVPE exhibits sufficient mechanical and chemical strength to maintain a suspended structure without a support layer and additionally withstands the selective removal of sacrificial oxide layers via wet etching. Therefore, the presented technique facilitates the fabrication of robust AlN suspended structures that are well suited for achieving group III-Nitride heteroepitaxial MEMS/NEMS systems on silicon substrates. 


\subsection{Experimental Method}

Figure 9-1 illustrates the process flow for the fabrication. First the sacrificial layer was formed by depositing a $\mathrm{SiO}_{\mathrm{x}}(x<2)$ film on silicon (111) substrates via Plasma Enhanced Chemical Vapor Deposition (PECVD) using an Oxford Plasma Lab 80 Plus system. The thickness of the sacrificial layer was varied from $200 \mathrm{~nm}$ to $800 \mathrm{~nm}$. $\mathrm{SiO}_{\mathrm{x}}$ is often used as a sacrificial layer in surface micro-machining because it can be selectively etched with respect to silicon $^{166}$. Standard Ultra Violet (UV) photolithography and wet etching techniques were used to create a striped pattern in the oxide layer. The width of the stripes ranged from $5 \mu \mathrm{m}$ to 110 $\mu \mathrm{m}$ and were oriented along the $<110>$ direction. Other stripe orientations were also investigated but no significant differences were observed. This work targeted structures with various dimensions in order to verify the structural stability. AlN films were grown on the patterned substrates in

an

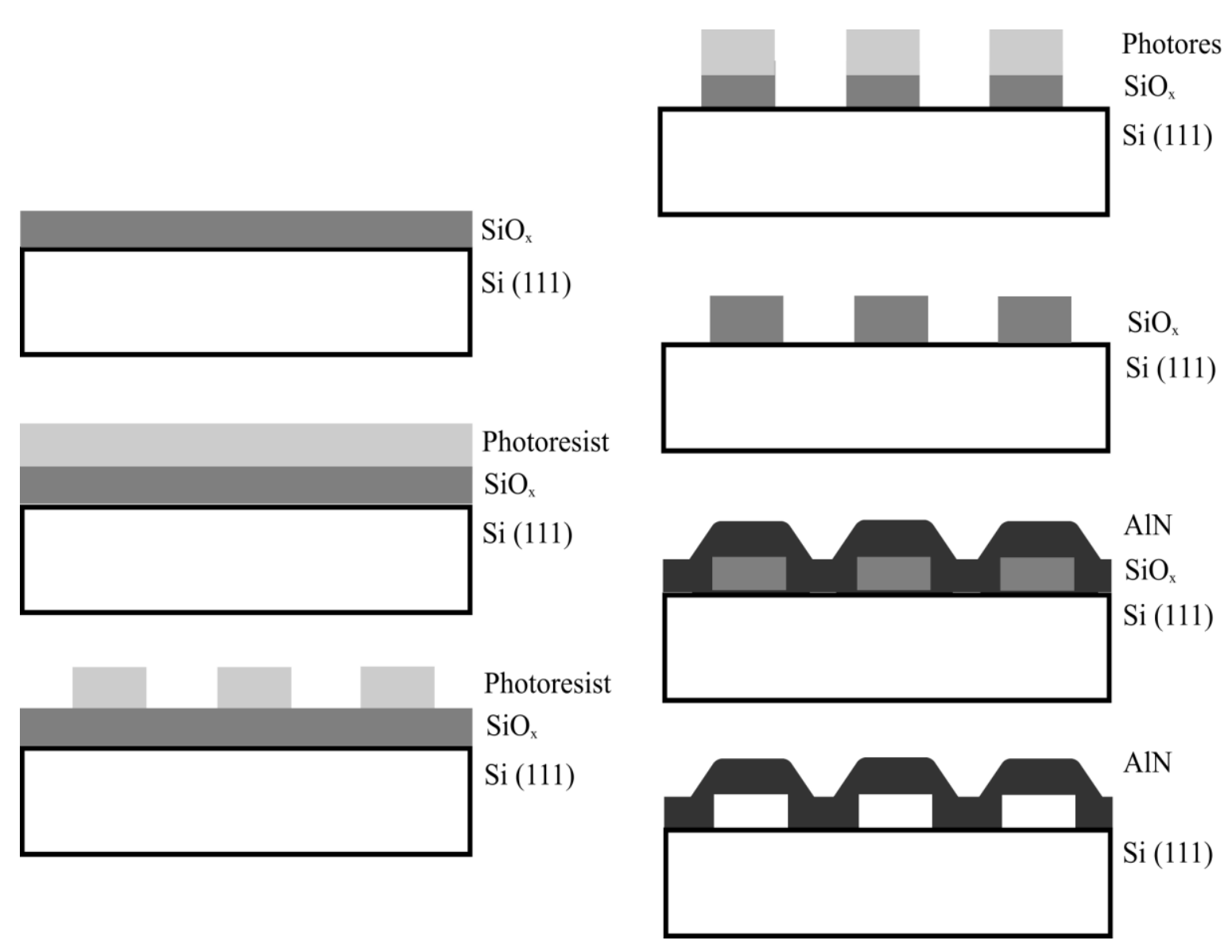

Figure 9-1 - Flow process for the fabrication of AlN suspended structures. 
Aixtron MOVPE horizontal reactor. Trimethylaluminum and ammonia were used as the precursors with typical flows around $12 \mu \mathrm{mol} / \mathrm{min}$ and $1.5 \mathrm{slm}$ respectively. Hydrogen was used as the carrier gas with the total flow into the reactor being approximately $9000 \mathrm{slm}$. All growths were conducted at a reactor pressure of 50 mbar and a temperature around $1100{ }^{\circ} \mathrm{C}$ resulting in an approximate growth rate of $4 \mathrm{~nm} / \mathrm{min}$. For each sample, the film thickness was approximately equal to the sacrificial layer thickness, ranging between $250 \mathrm{~nm}$ to $800 \mathrm{~nm}$. Finally, the wafer was diced into smaller pieces by cutting the sample perpendicular to the stripes. The pieces were etched in a 10:1 Buffered Oxide Etch (BOE) to remove the oxide and dried. It is widely known that the crystalline quality affects the etch rate of AIN in various acids ${ }^{154,155,156}$. Since crystalline AlN has been reported to be resistant to attack by hydrofluoric acid ${ }^{167,168}$ and hydrofluoric acid is known to etch $\mathrm{SiO}_{\mathrm{x}}$ uniformly selective to silicon, $\mathrm{BOE}$ was used in this work. It should be noted that etch rate, however, depends strongly on the method of $\mathrm{SiO}_{\mathrm{x}}$ deposition due to variations in stoichiometry, density, and impurities ${ }^{166}$. $\mathrm{SiO}_{2}$ deposited via PECVD has a reported etch rate in $\mathrm{BOE}$ between $120-200 \mathrm{~nm} / \mathrm{min}^{166}$. As this work used $\mathrm{SiO}_{\mathrm{x}}(x<2)$ deposited via PECVD, it is expected that the etch rate of the sacrificial layer is faster, but it has not been determined yet.

\subsection{Characterization}

The samples were analyzed in a Hitachi S4700 Scanning Electron Microscope (SEM) in order to extract structural information. Figure 9-2 shows SEM images of the AlN film grown over a $5 \mu \mathrm{m}$ wide $\mathrm{SiO}_{\mathrm{x}}$ stripe and the corresponding suspended structure after wet etching. The air gap has a height of approximately $200 \mathrm{~nm}$ is and equal to the thickness of the sacrificial layer while the width is equal to the feature size on the lithographic mask used. Figure 9-3 shows a variety of suspended structures of different dimensions. Figure 9-3a contains an array of $5 \mu \mathrm{m}$ wide structures with an $5 \mu \mathrm{m}$ spacing. Fig. $3 \mathrm{~b}$ shows the corners of the suspended structure in Figure 9-3a in more detail. Figure 9-3c illustrates a $24 \mu \mathrm{m}$ wide structure having an air gap of $600 \mathrm{~nm}$ while Figure 9-3d is a $90 \mu \mathrm{m}$ wide structure with an air gap of $500 \mathrm{~nm}$. The structures in Figure 9-3c and Figure 9-3d have low angled sidewalls when compared to those shown in Figure 9-3a and Figure 9-3b due to the combined effect of the thicker $\mathrm{SiO}_{\mathrm{x}}$ layer and wet etching process prior to the AIN growth. Despite minor differences such as these, similar results were obtained for the various combinations of feature sizes and sacrificial layer thicknesses. Energy 

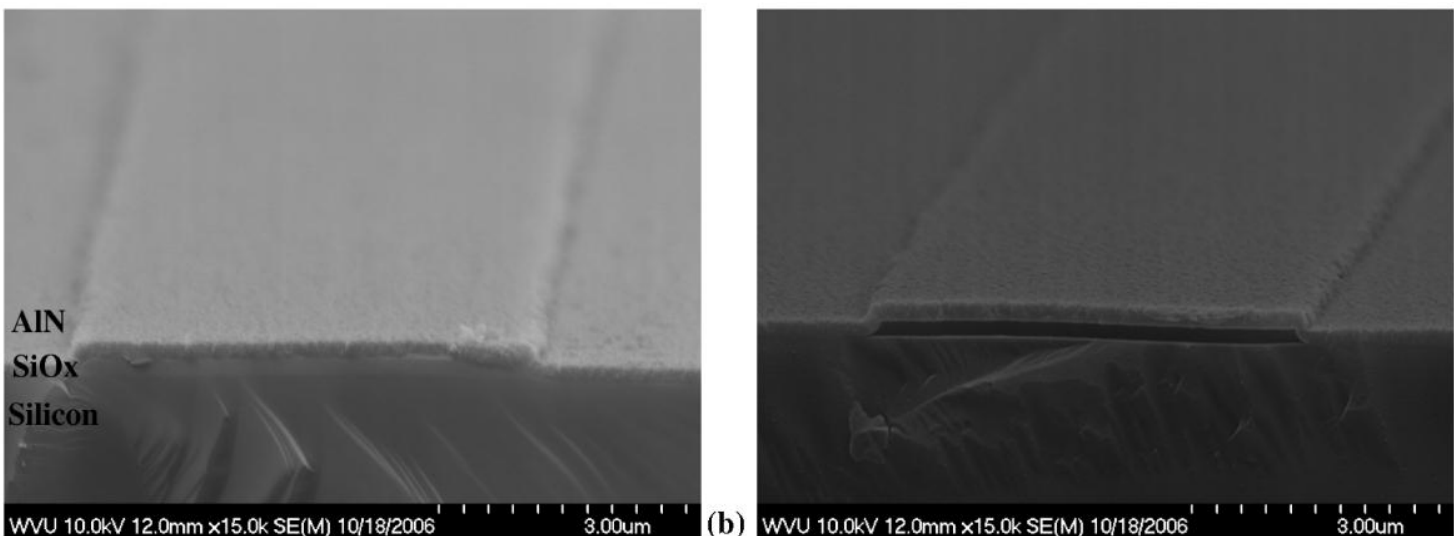

Figure 9-2 - (a) AlN grown on $5 \mu \mathrm{m}$ wide SiO stripe with $200 \mathrm{~nm}$ thickness (b) Free standing AlN micro-structures after removal of $\mathrm{SiO}_{x}$ sacrificial layer. Air gap height: 200nm, AlN thickness: 250nm, Width: $5 \mu \mathrm{m}$.
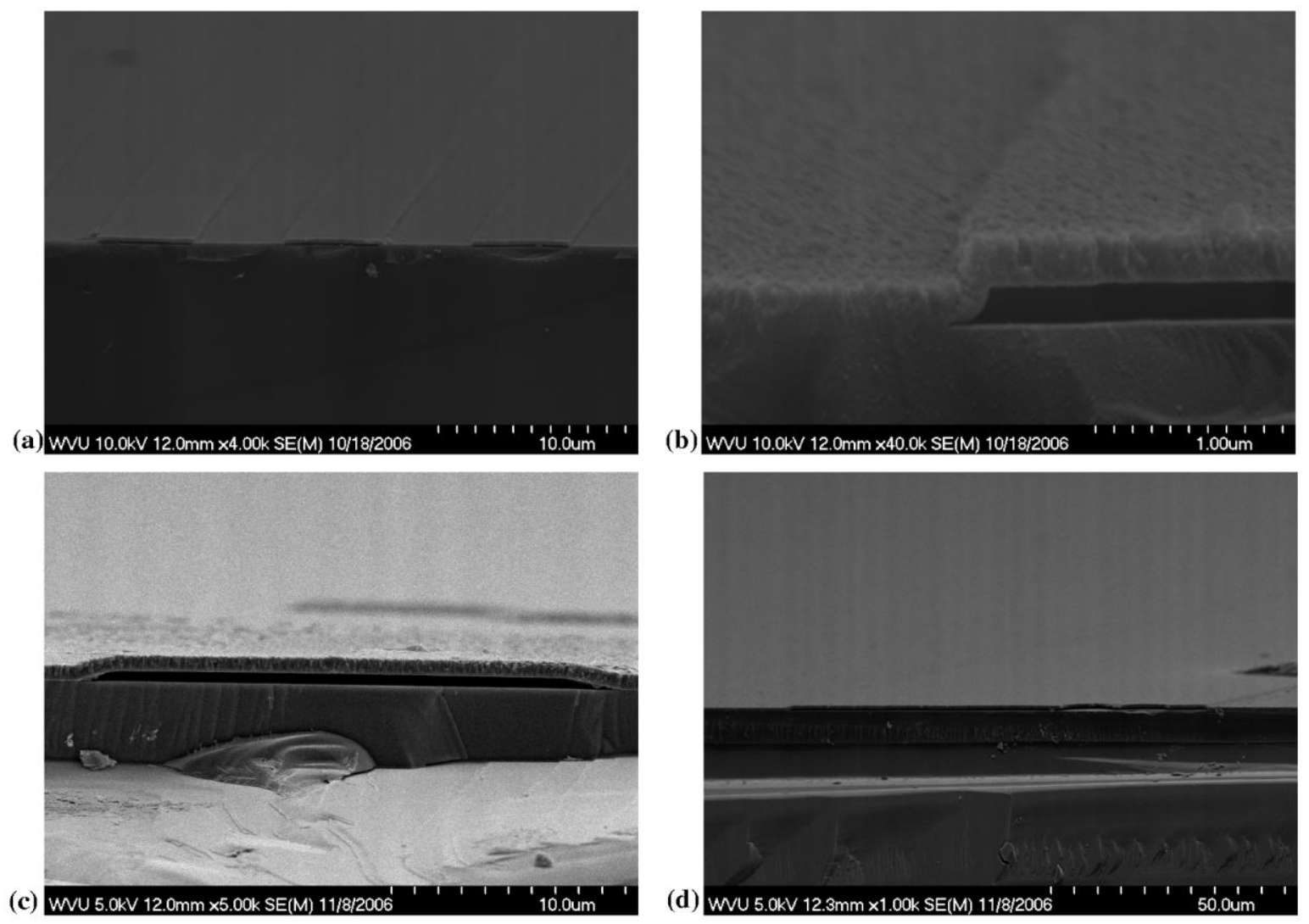

Figure 9-3 - (a) Array of $5 \mu \mathrm{m}$ wide AlN micro-structures. air gap height: $200 \mathrm{~nm}$ and AlN thickness: $250 \mathrm{~nm}$, (b) Closer view of corner of AlN micro-structure in (a), (c) AlN microstructure, air gap height: 500nm, AlN thickness: 500nm, width: $24 \mu \mathrm{m}$. (d) AlN microstructure, air gap height: 600nm, AlN thickness: 500nm, width: $90 \mu \mathrm{m}$ 
Dispersive X-Ray Spectroscopy (EDS) was used to investigate the removal of the oxide layer under the AlN layer. Measurements taken several microns from the edge of a fresh cut did not indicate any underlying oxide.

These results demonstrate the growth and fabrication of AlN micro-structures using MOVPE and silicon surface micro-machining techniques. When grown via MOVPE, AIN nucleates easily on dielectrics, such as silicon oxide and silicon nitride, due to the short diffusion length and large sticking coefficient of the Al species ${ }^{169,43}$. Therefore, AlN films grown on the sacrificial oxide layers are often of poor crystalline quality and can be removed by wet etching ${ }^{170}$. Since it is known that for a given temperature, slower growth rates almost invariably produce higher quality films ${ }^{171}$, this work targeted slow growth rates in order to promote surface diffusion and improve the crystal quality of the AlN on the oxide layer. Conditions used resulted in an AlN growth rate of 4 seconds per monolayer, which is significantly slower than typical nitride growth rates of $0.5-2.5$ seconds per monolayer. It can therefore be speculated that the growth rate used in this work allows $\mathrm{AlN}$ grown on the $\mathrm{SiO}_{\mathrm{x}}$ surface to self-orient along the cplane and therefore become etch resistant to BOE. The relationship between the quality of the AlN grown on the oxide layers and the growth conditions is currently being investigated.

In summary, a process for fabricating robust AlN micro-structures by combining silicon surface micromachining techniques and MOVPE growth has been demonstrated. Conditions were identified that result in the growth of etch-resistant polycrystalline AlN films on $\mathrm{SiO}_{x}$ sacrificial layers. Etching techniques were developed to release the AlN from the sacrificial layer to fabricate unsupported, suspended structures for use as mico-bridges or channels. Due to the structural integrity of the material and simplicity of the fabrication process, complex, multilevel devices could be fabricated. Furthermore, the versatility of this technique lends itself to the fabrication of nano-sized suspended structures by reducing the thickness and width of the sacrificial layer, provided a suitable etchant is identified for complete removal of the sacrificial layer. 


\section{CHA P T ER 10 - CONCLUSIONSAND FUTURE WORK}

III-Nitride materials continue to attract attention for use in optoelectronic and electronic devices. With the ability to engineer the emission across the visible regime, this material system is of particular use for high brightness visible wavelength LEDs. Within LED fabrication, DBRs are required for increased light extraction as well for cavity based device ${ }^{6}$ such as RCLEDs ${ }^{74}$ and VCSELs $^{172}$. The fabrication of III-Nitride based DBRs has been hindered by material issues and growth challenges. In particular, the lattice and thermal mismatch with the III-Nitride system gives rise to defects and/or cracking when high refractive index contrast materials, such as AlN and $\mathrm{GaN}$, are used in superlattice structures ${ }^{3}$. In this work, two different strain relieving techniques have been investigated in order to eliminate cracking in high $\mathrm{Al}$ content $\mathrm{Al}_{x} \mathrm{Ga}_{1 \text { - }}$ ${ }_{x} \mathrm{~N} / \mathrm{GaN}$ DBRs. In particular, indium was investigated as a surfactant and a digital alloy growth technique was developed and integrated in the DBRs for additional strain relief.

Indium has been reported to improve the surface morphology and crystalline properties of $\mathrm{Al}_{x} \mathrm{Ga}_{1-x} \mathrm{~N}$ alloys in addition to eliminating cracking in single layer films and MQW structures $^{57,58,60,61}$. In this work, it was used as a surfactant to minimize cracking in AlN/GaN DBRs. AlN/GaN DBRs have been fabricated and control over the center reflectivity wavelength was demonstrated by modifying the individual layer thickness. Trimethylindium was introduced during the growth of the AIN in the DBR and its influence on strain and cracking was investigated through evaluation of the surface crack density and also XRD measurements. Under optimal conditions, the surface cracking in a six period structure was reduced by a factor of two using indium during growth.

The second growth technique focused on developing a digital growth technique for $\mathrm{Al}_{x} \mathrm{Ga}_{1-x} \mathrm{~N}$ alloys. Digital alloy growth techniques utilize short period superlattice structures to alleviate the previously mentioned growth challenges including but not limited to, parasitic reactions of precursors, complex growth kinetics of multiple species, and phase separation. Furthermore, superlattice layers have long been used as strain relieving layers in layers to engineering the stress in III-Nitride layers ${ }^{70,71}$. They have also been employed in AIN/GaN DBRs in order to suppress cracking ${ }^{76,78}$. In this work, the digital alloy growth technique has been used to integrate a short period superlattice into each period of the DBR structure. This 
allowed for a decrease in the total thickness of the DBR and complexity of the structure as compared to the superlattice insertion layers. Growth conditions which yield high quality $\mathrm{Al}_{x} \mathrm{Ga}_{1-x} \mathrm{~N}$ digital alloys have been developed. The conditions determined maintain the superlattice structure as indicated by the presence of satellite peaks in the XRD measurements. Alloys with AlN mole fractions ranging from 0 to 1 have been grown by changing the AlN thickness within the sueprlattice. As these structures are crack-free, XRD measurements have been used to evaluate the difference in the strain evolution.

Digital alloys with an $\mathrm{Al}$ concentration around $90 \%$ have been integrated in $\mathrm{Al}_{x} \mathrm{Ga}_{1 \text { - }}$ ${ }_{x} \mathrm{~N} / \mathrm{GaN}$ DBR in place of the AIN layer. DBRs with up to 25 periods have been fabricated and exhibit $\sim 94 \%$ reflectivity at the targeted wavelength. 2 " substrates have been prepared and are largely crack-free with the only visible cracks lie along the edge of the 2" wafer. After a $600 \mathrm{~nm}$ overgrowth of $\mathrm{GaN}$, these structures exhibit $<1 \mathrm{~nm}$ surface roughness and are suitable for integration with active devices. In this work, LEDs have been grown on the DBR substrates and exhibit a considerable increase in light extraction as compared to a conventional LED on a GaN epilayer.

Future work includes integrating higher reflectivity DBRs with visible LEDs in order to increase the light extraction further. For the fabrication of RCLEDs, the use of a highly reflective top $p$-contact or dielectric DBR will be integrated with the structure.

Other areas of interest for future work include doping studies on the digital alloys. There is a considerable interest in UV emitters for applications, including but not limited to, biological detection, water purification, sterilization, and medical diagnostics ${ }^{173}$. Despite the recent improvement in efficiency of visible wavelength nitride-based emitters, the UV devices are still hindered by poor efficiencies and low output power. One of the challenges limiting efficiency includes the difficulty of achieving $p$-type doping in high aluminum content $\mathrm{Al}_{x} \mathrm{Ga}_{1-x} \mathrm{~N}$ alloys ${ }^{174}$. Efficient doping becomes even more challenging in wider bandgap $\mathrm{Al}_{x} \mathrm{Ga}_{1-x} \mathrm{~N}$ layers ${ }^{174}$ as the activation energy can exceed $300 \mathrm{meV}$ for high $\mathrm{Al}$ concentrations ${ }^{175}$. As a result, $p$-type $\mathrm{Al}_{x} \mathrm{Ga}_{1-}$ ${ }_{x} \mathrm{~N}$ films are characterized by low carrier concentrations and mobilities which result in high device operating voltages and contact resistances ${ }^{176}$. One technique that has shown promising results for overcoming the high activation energy is the use of $\mathrm{Mg}$ doped $\mathrm{Al}_{x} \mathrm{Ga}_{1-x} \mathrm{~N} / \mathrm{GaN}$ short period superlattice structures ${ }^{174,176}$. The improvement in ionization efficiency results from the 
valence band edge oscillations induced by the compositional modulation of the superlattice ${ }^{177}$. This allows the acceptors to ionize where the band edge is below the Fermi level ${ }^{177}$ creating a sheet of holes parallel to the interface. While there have been many reports of enhanced carrier concentrations, it is typically for $\mathrm{Al}_{x} \mathrm{Ga}_{1-x} \mathrm{~N}$ films with $\mathrm{Al}$ compositions of 0.40 or less ${ }^{175}$. There are fewer reports extending this technique into high Al content alloys, $x>0.50$. Therefore it is of considerable interest to develop the doping techniques for digital alloys for integration in devices. Final areas of interest include integrating the digital alloys in electronic structures such as High Electron Mobility Transistors (HEMTs) as an alternative to random alloys. 
The following steps can be used to calibrate the Luxtron temperature controller.

1. Under the conditions used for the pyrometer test, take the MOVPE temperature to $850^{\circ} \mathrm{C}$.

2. Measure the temperature of the silicon (100) wafer with the infrared pyrometer from the top viewport on the reactor. Please note, the ceiling will have to be removed for the measurement.

3. If there is a discrepancy in the measured temperature and the temperature displayed in the Luxtron, then enter the Luxtron settings to reach the Sensor Calibration menu.

4. Reset the Sensor Calibration to the actual surface temperature that is measured with the pyrometer. ***Please note that if the setpoint and measured temperature differ significantly, use incremental values. For example, if the setpoint is $850{ }^{\circ} \mathrm{C}$ and the temperature measured with the pyrometer is $760{ }^{\circ} \mathrm{C}$. The Sensor Calibration menu will initially display $850{ }^{\circ} \mathrm{C}$. Change this to $825^{\circ} \mathrm{C}$. As the setpoint in the CACE software is still $850{ }^{\circ} \mathrm{C}$ the power will increase and heat the reactor until the Luxtron reads $850{ }^{\circ} \mathrm{C}$. At this point, the reading with the pyrometer should increase $25{ }^{\circ} \mathrm{C}$ to $785^{\circ} \mathrm{C}$. Repeat this in increments of $25^{\circ}$ until the pyrometer reading reaches $850^{\circ} \mathrm{C}$.

5. Note the sensitivity factors in the log book.

6. Perform a full pyrometer test to verify the temperature over a wide range of values.

The following settings should be used. The sensor factor may change with the above calibration process.

\begin{tabular}{|l|l|l|}
\hline & Ch 1 (Si) & Ch 5 (InGaAs) \\
\hline Sensor Type & Light Pipe & Light Pipe \\
\hline Sensor Factor & $3.729112 \mathrm{E} 00$ & $1.472112 \mathrm{E} 00$ \\
\hline Bandwidth & No Filter & Auto \\
\hline Gain & Auto & Auto \\
\hline Emissivity & 1 & 1 \\
\hline Sensor Calibration & 325 & 100 \\
\hline
\end{tabular}

The output settings should be set to the following:

\begin{tabular}{|l|l|}
\hline Crossover & $*$ \\
\hline Temperature & 600 \\
\hline Low Temperature Channel & 5 \\
\hline High Temperature Channel & 1 \\
\hline
\end{tabular}


The output function should be set to the following:

\begin{tabular}{|l|l|}
\hline Low Temperature & 100 \\
\hline Low Current & $4 \mathrm{~mA}$ \\
\hline High Temperature & 1700 \\
\hline High Current & $20 \mathrm{~mA}$ \\
\hline
\end{tabular}


This procedure is used to verify the susceptor temperature.

1. Remove the EpiRAS optics.

2. Remove the quartz ceiling from the reactor.

3. Load a clean Silicon (100) wafer.

4. Pump down the chamber to 200 mbar.

5. Set the flows to the following:

\begin{tabular}{|l|l|l|}
\hline MFC & Flow $(\mathbf{s c c m})$ & Gas \\
\hline Run Hydride & 5 & $\mathrm{H} 2$ \\
\hline Push Hydride & 200 & $\mathrm{H} 2$ \\
\hline Run MO & 750 & $\mathrm{H} 2$ \\
\hline Push MO & 750 & $\mathrm{~N} 2$ \\
\hline Liner Purge & 2500 & $\mathrm{H} 2$ \\
\hline Rotation & 200 & $\mathrm{~N} 2$ \\
\hline Window Purge & 250 & $\mathrm{H} 2$ \\
\hline Lightpipe & 50 & $\mathrm{H} 2$ \\
\hline
\end{tabular}

6. Increase the temperature to $600{ }^{\circ} \mathrm{C}$ slowly.

7. Set the emissivity of the pyrometer to 0.69 and position so it is aimed through the top viewport of the reactor.

8. Measure the temperature with the pyrometer and compare to the setpoint over a wide range of temperature.

9. If necessary, adjust the sensitivity settings in the Luxtron controller as outlined in Appendix A. 
This procedure is used to verify the operation of the MFC.

1. Pump down to 0 mbar.

2. Open the throttle valve.

3. Close the Nitrogen supply.

4. Set Pressure Controllers to 0 and Mass Flow Controllers to maximum.

5. Wait until the base pressure has reached a minimum.

6. Close the throttle valve. The pressure should remain at the minimum.

7. Set the flow on the MFC under investigation to the desired value.

8. Open the gas supply to that MFC. The pressure in the reactor will start to increase.

9. Set the reactor pressure to 1000 mbar.

10. Note the rate at which the pressure increases.

11. Repeat steps 1-10 for a different MFC to compare.

Note: MFCs may be calibrated for different gasses. 


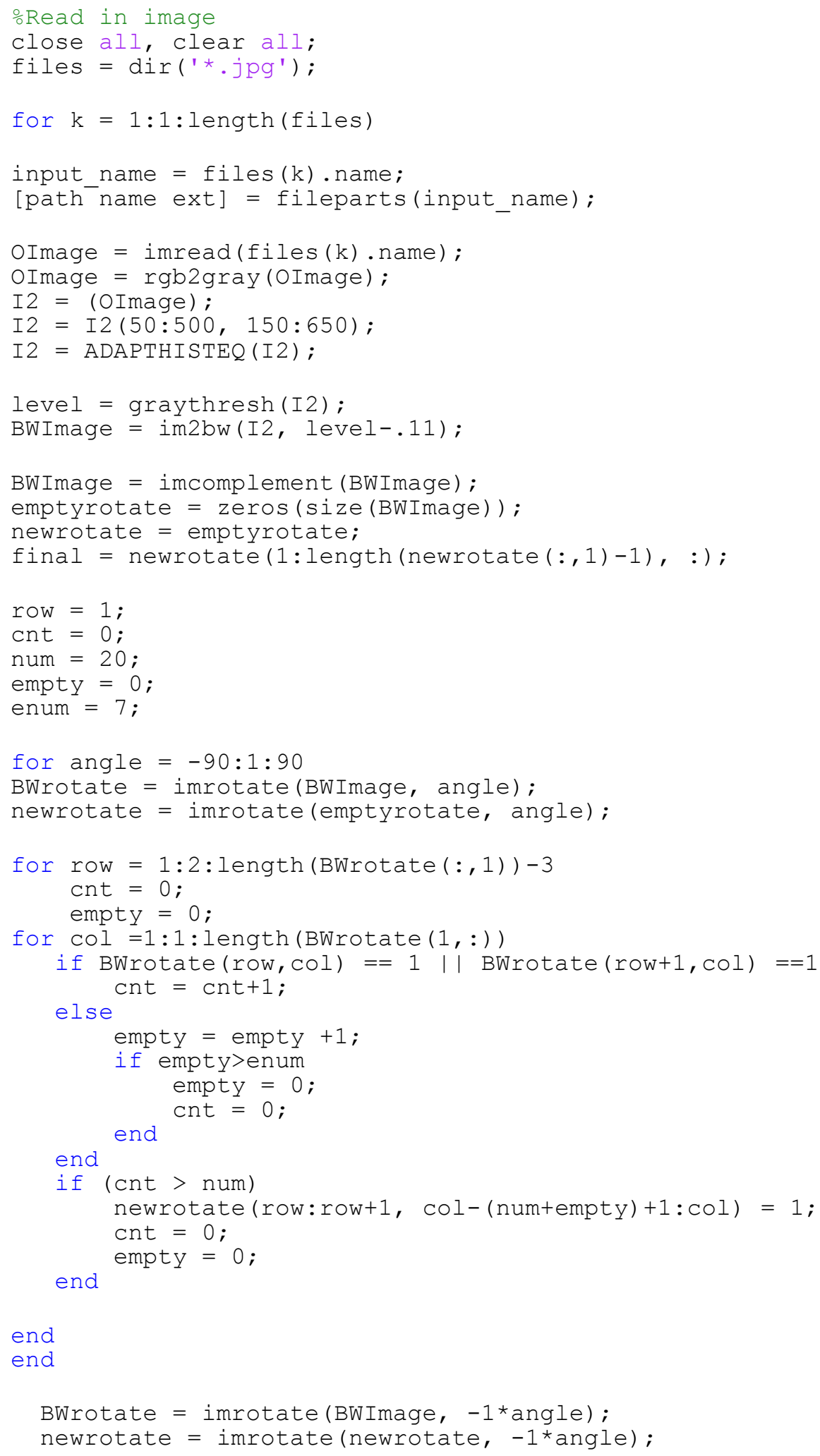




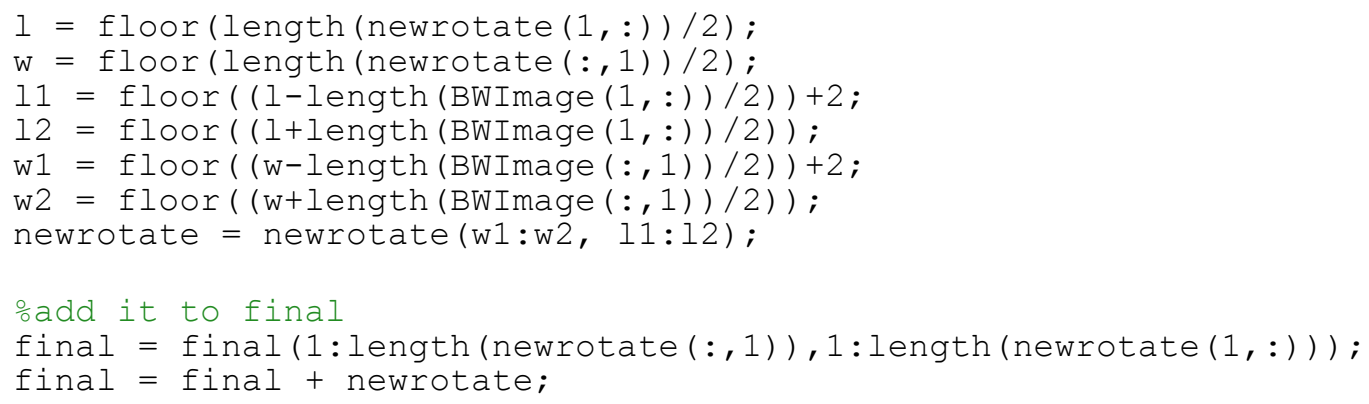


APPENDIX E: MATLAB CODE FOR DBR RESPONSE A T O B L I Q UE A N G L S OF INCIDEN C E

\section{FOR TE MODES:}

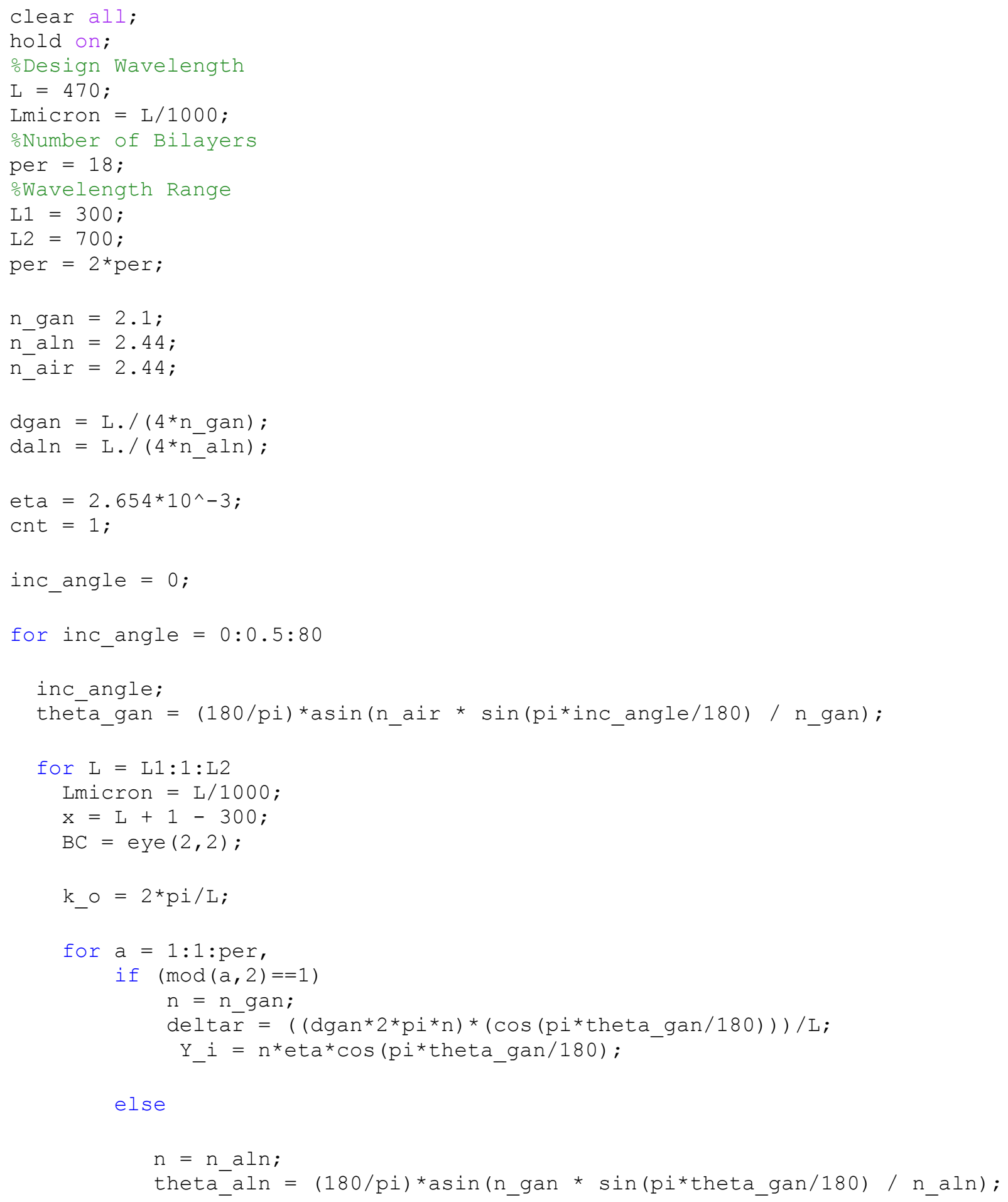




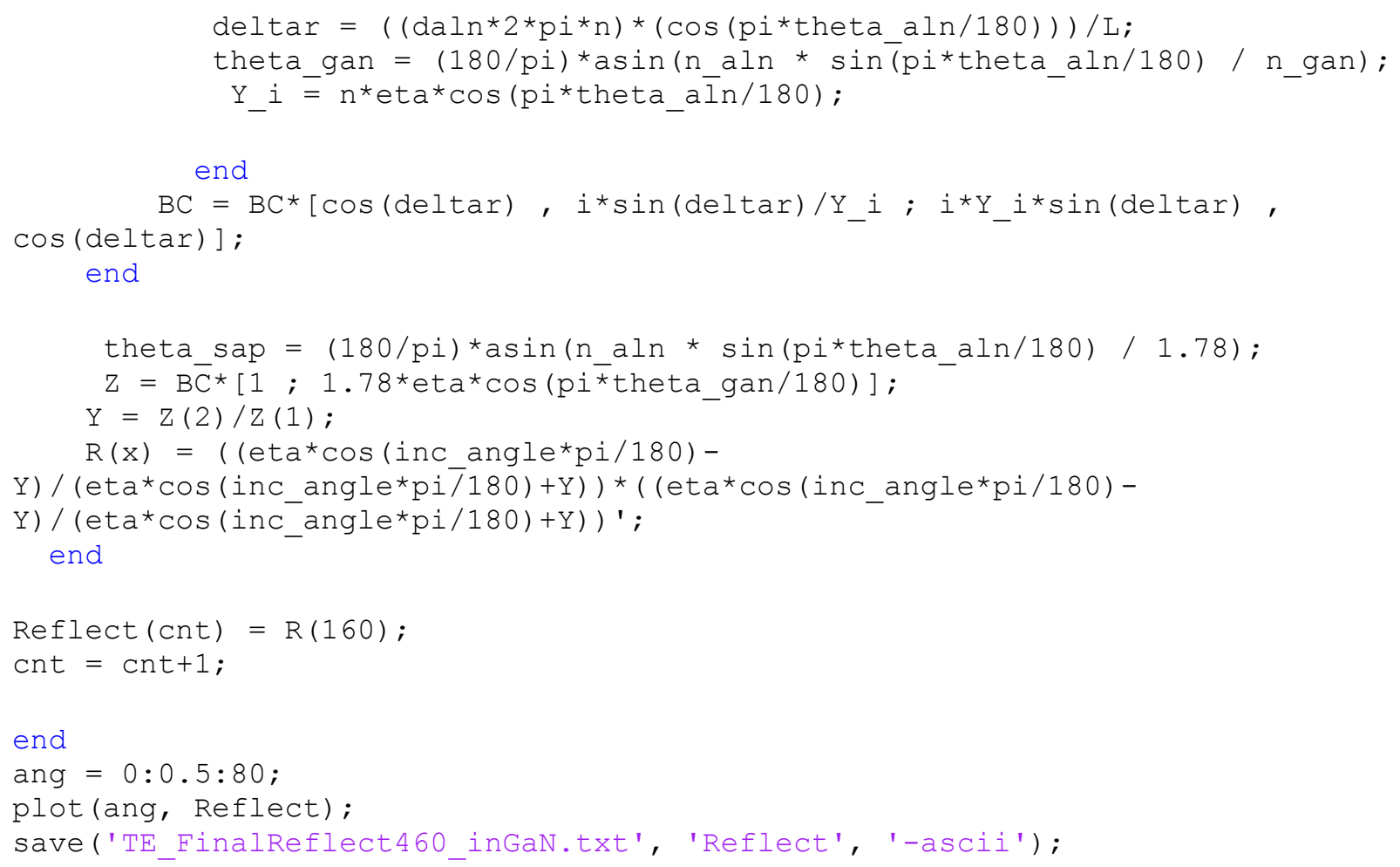

\section{FOR TM MODES:}

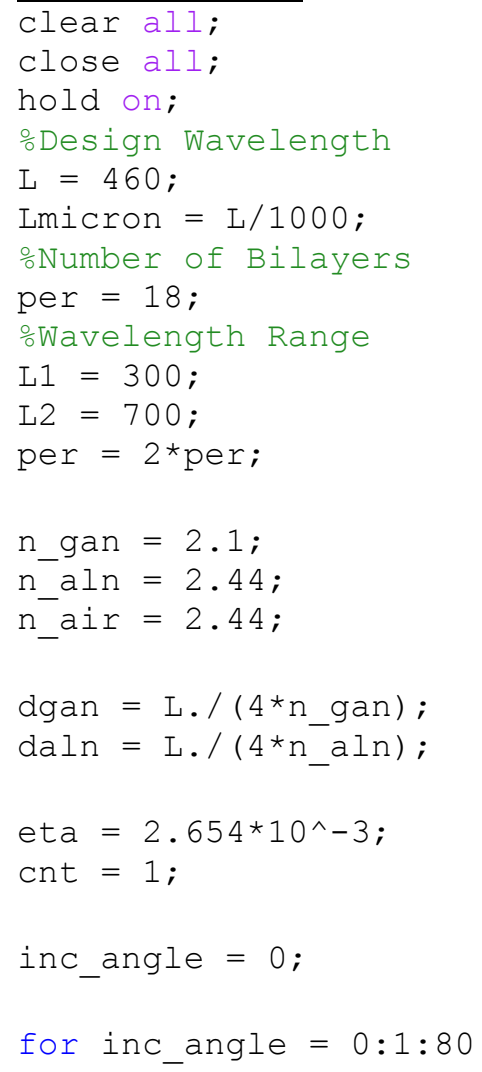




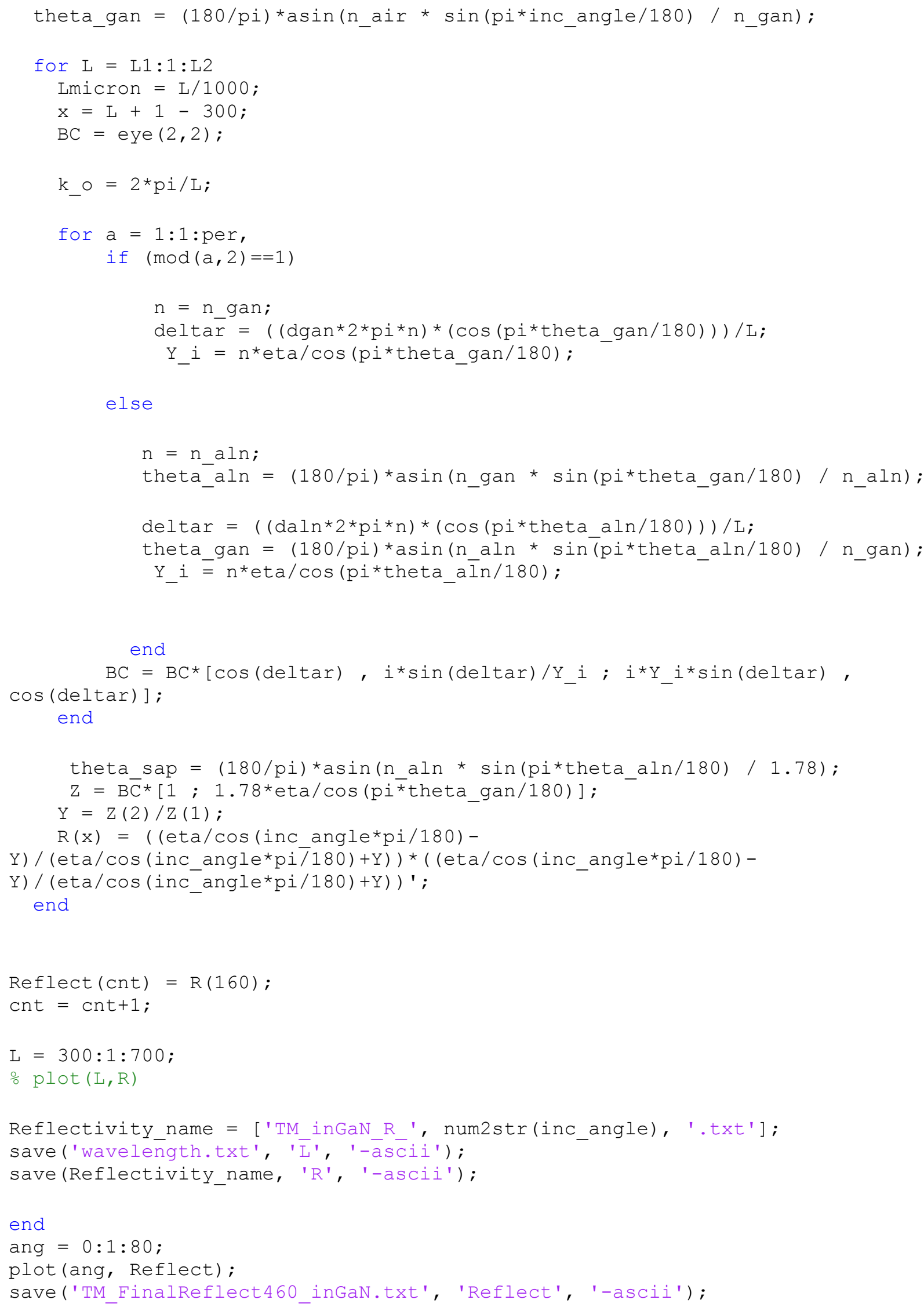




\section{$\underline{\text { B I B L I O G R A P H Y }}$}

1 P. Gibart. "Metal organic vapour phase epitaxy of GaN and lateral overgrowth," Rep. Prog. Phys. 67, 667 (2004).

2 O. Ambacher. "Growth and Applications of Group III-Nitrides," J. Phys. D: Appl. Phys. 31, 2653 (1998).

3 S. J. Hearne, J. Han, S. R. Lee, J. A. Floro, D. M. Follstaedt, E. Chason, and I. S. T. Tsong. "Brittle-ductile relaxation kinetics of strained AlGN/GaN heterostructures," Appl. Phys. Lett. 76, 1534 (2000).

4 S. Srinivasan, M. Stevens, F. A. Ponce, H. Omiya and T. Mukai. "Carrier dynamics and electrostative potential variation in InGaN quantum wells grown on (11-22) GaN pyramidal planes," Appl. Phys. Lett. 89, 231908 (2006).

5 F. K. Yam and Z. Hassan. "InGaN: An overview of the growth kinetics, physical properties and emission mechanisms," Superlattices Microstruct. 43, 1 (2008).

6 S. O. Kasap. Optoelectronics and Photonics Principles and Practices. Upper Saddle River: Prentice Hall, 2001.

7 Department of Energy, "Solid State Lighting Research and Development: Multi Year Program Plan," March 2010.

8 Z. Qin, Z. Chen, Y. Tong, S. Lu, and G. Zhang. "Estimation of InN phase inclusion in InGaN films grown by MOVPE,” Appl. Phys. A. 74, 655 (2002)

9 D. Gerthsen, E. Hahn, B. Neubauer, V. Potin, A. Rosenauer, and M. Schowalter. "Indium distribution in epitaxially grown $\mathrm{InGaN}$ layers analyzed by transmission electron microscopy, " Phys. Stat. Sol (c) 0, 1668 (2003)

10 J. Wu, W. Walukiewicz, K.M. Yu, W. Shan, J.W. Ager III, E.E. Haller, Hai Lu, William J. Schaff, W. K. Metzger, and Sarah Kurtz. "Superior radiation resistance of InGaN alloys: Full-solar-spectrum photovoltaic material system” J. Appl. Phys. 94, 6477 (2003).

11 Yoshitaka Taniyasu, Makoto Kasu, and Toshiki Makimoto. "An aluminum nitride lightemitting diode with a wavelength of 210 nm," Nature Letters 441, 325 (2006).

12 Georges A. Wagnieres, Willem M. Star, and Brian C. Wilson. "In Vivo Fluorescence Spectroscopy and Imaging for Oncological Applications," Photochemistry and Photobiology, 68, 603-632 (1998).

13 Nikhil Ganesh, Ian D. Block, and Brian T. Cunningham. "Near ultraviolet-wavelength photonic crystal biosensor with enhanced surface to bulk sensitivity ratio," Appl. Phys. Lett. 89, 023901 (2006). 
14 V.V.R. Sai, Tapanendu Kundu, Chitra Deshmukh, Susan Titus, Pradeep Kumar, and Soumyo Mukherji. "Label-free fiber optic biosensor based on evanescent wave absorbance at 280 nm," Sensors and Actuators B 143, 724-730 (2010).

15 Jianyu Deng, Yuriy Bilenko, Alex Lunev, Xuhong Hu, Thomas Katona, Jianping Zhang, Michael Shur, and Remis Gaska. "247 nm Ultra-Violet Light Emitting Diodes," Jap. J. Appl. Phys. 46, L263-L264 (2007).

16 M. Asif Khan, M. Shatalov, H. P. Maruska, H. M. Wang, and E. Kuokstis. "III-Nitride UV Device,” Jap. J. Appl. Phys. 44, 7191-7206 (2005).

17 Umesh K. Mishra, Primit Parikh, and Yi-Feng Wu. "AlGaN/GaN HEMTs - An Overview of Device Operation and Applications,” Proc. IEEE 90, 1022 (2002).

18 Gaudenzio Meneghesso, Matteo Meneghini, Augusto Tazzoli, Nicolo Ronchi, Antonio Stocco, Alessandro Chini, and Enrico Zanoni. "Reliability issues of Gallium Nitride High Electron Mobility Transistors." International Journal of Microwave and Wireless Technologies, 2, 39-50 (2010).

19 W. D. Hu, X. S. Chen, F. Yin, J. B. Zhang, and W. Lu. "Two-dimensional transient simulations of drain lag and current collapse in GaN-based high-electron-mobility transistors," J. Appl. Phys. 105, 084502 (2009).

20 Kelson D. Chabak, James K. Gillespie, Virginia Miller, Antonio Crespo, Jason Roussos, manuel Trejo, Dennis E. Walker Jr, Glen D. Via, Gregg H. Jessen, John Wasserbauer, Firooz Faili, Dubravko I. Babic, Daniel Francis, and Felix Ejeckam."Full Wafer Characterization of AlGaN/GaN HEMTs on Free Standing CVD Diamon Substrates," IEEE Electron Device Letters 31, 99 (2010).

21 Xiaobin Xu, Vibhu Jindal, Fatemeh Shahedipour-Sandvik, Magnus Bergkvist, Nathaniel C Cady. "Direct immobilization and hybridization of DNA on group III nitride semiconductors," Appl. Surf. Sci. 255, 5905-5909 (2009).

22 V. Cimalla, J. Pezoldt, and O. Ambacher. "GroupIII nitride and SiC based MEMs and NEMS: materials properties, technology and application,” J. Phys. D: Appl. Phys. 40, 63866436 (2007).

23 R.C. Turner, P.A. Fuierer, R.E. Newnham, T.R. Shrout. "Materials for high temperature acoustic and vibration sensors: A review ,"Appl. Acoust. 41, 299 (1994).

24 Ji-Myon Lee, Chul Huh, Dong-Joon Kim, and Seong-Ju Park. Semicond. "Dry-etch damage and its recovery in InGaN/GaN multi-quantum-well light-emitting diodes,"Sci. Technol. 18 ,530-534 (2003).

25 M. Bockowski. "Review: Bulk growth of gallium nitride: challenges and difficulties," Cryst. Res. Technol. 42, 1162 (2007). 
26 G. Stringfellow. Organometallic Vapor-Phase Epitaxy Theory and Practice. Academic Press:California 1999.

27 N. Amano, N. Sawaki, I. Akasaki, and Y. Toyoda. "Metalorganic Vapor Phase Epitaxial Growth of High Quality GaN Film Using an AlN Buffer Layer," Appl. Phys. Lett. 48, 353 (1996).

28 K. Hiramatsu, S. Itoh, H. Amano, I. Akasaki, N. Kuwano, T. Shiraishi, and K. Oki. "Growth mechanism of GaN grown on sapphire with AlN buffer layer by MOVPE," J. Cryst. Growth 115, 628 (1991).

29 W. Wang, J. Gong, C. Wang, W. Liao, J. He, Y. Chi, and J. Shi, "Characteristics and Interactions of Threading Dislocations in GaN Films Grown on (0001) Sapphire Substrates With or Without Short-Period Superlattice Insertion,” Jap. J. Appl. Phys. 45, 6888 (2006).

30 B. Beaumont, P. Vennegues, and P. Gibart, "Epitaixial Lateral Overgrowth of GaN," Phys. Stat. Sol. (b) 227, 1 (2001).

31 V. Narayanan, K. Lorenz, W. Kim, and S. Mahajan, "Origins of Threading Dislocations in GaN Epitaxial Layers Grown on Sapphire by Metalorganic Chemical Vapor Deposition," Appl. Phys. Lett. 78, 1544 (2001).

32 P. de Mierry, B. Beaumont, E. Feltin, H.P.D. Schenk, Pierre Gibart, F. Jomard, S. Rushworth, L. Smith and R. Odedra, "Influence of the Mg precursor on the incorporation of Mg in MOVPE grown GaN,” MRS Internet J. Nitride Semicond. Res. 5, 8 (2000).

33 V. Bousquet, P. Vennegues, B. Beaumont, M. Vaille, and P. Gibart, "TEM Study of the Behavior of Dislocaitons during ELO of GaN," Phys. Stat. Sol. (b) 216, 691 (1999).

34 Kazumasa Hiramatsu. "Epitaxial lateral overgrowth techniques used in group III nitride epitaxy" J. Phys.: Condens. Matter 13, 6961-6975 (2001).

35 K. Hiramatsu, K. Nishiyama, A. Motogaito, H. Miyake, Y. Iyechika, T. Maeda. "Recent Progress in Selective Area Growth and Epitaxial Lateral Overgrowth of III-Nitrides:Effects of Reactor Pressure in MOVPE Growth.” Phys. Stat. Sol. (a) 176, 535 (1999).

36 G. Feng, Y. Fu, J. S. Xia, J. J. Zhu, X. M. Shen, D. G. Zhao, H. Yang, J. W. Liang. "Mechanisms of the sidewall facet evolution in lateral epitaxial overgrowth of $\mathrm{GaN}$ by MOCVD.” J. Phys. D: Appl. Phys. 35, 2731 (2002).

37 X. Zhang, P. D. Dapkus, D. H. Rich. "Lateral epitaxy overgrowth of $\mathrm{GaN}$ with $\mathrm{NH}_{3}$ flow rate modulation." Appl. Phys. Lett. 77, 10 (2000).

38 Akira Usui, Haruo Sunakawa, Akira Sakai, and A. Atsushi Yamaguchi. "Thick GaN Epitaxial Growth with Low Dislocation Density by Hydride Vapor Phase Epitaxy" Jpn. J. Appl. Phys. 36 L899 (1999). 
39 Tsvetanka S. Zheleva, Waeil M. Ashmawi, Ok-Hyun Nam, and Robert F. Davis. "Thermal mismatch stress relaxation via lateral epitaxy in selectively grown GaN structures." Appl. Phys. Lett. 74, 2492 (1999).

Duanjun Cai, Fuchun Xu, Junyong Kang, Pierre Gibar, and Bernard Beaumont. "Highspatial-resolution strain measurements by Auger electron spectroscopy in epitaxial-lateralovergrowth GaN.” Appl. Phys. Lett. 86, 211917 (2005).

41 Suk Choi, Hee Jin Kim, Jae-Hyun Ryou, and Russell D. Dupuis, "Digitally alloyed modulated precursor flow epitaxial growth of AlGaN layers with AlN and AlGaN monolayers," J. Cryst. Growth 311, 3252-3256 (2009).

42 M. E. Hawkridge, Z. Liliental-Weber, Hee Jin Kim, Suk Choi, Dongwon Yoo, Jae-Hyun Ryou, and Russell D. Dupuis, "The structural quality of $\mathrm{Al}_{\mathrm{x}} \mathrm{Ga}_{1-\mathrm{x}} \mathrm{N}$ epitaxial layers grown by digitally alloyed modulated precursor epitaxy determined by transmission electron microscopy," Appl. Phys. Lett. 94, 071905 (2009).

43 Steven J. Pearton ed. Optoelectronic Properties of Semiconductors and Superlattics GaN and Related Materials II. Canada: Gordon and Breach Science Publishers 2000.

44 Engin Arslan, Mustafa K Ozturk, Ali Teke, Suleyman Ozcelik, and Ekmel Ozbay, "Buffer optimization for crack-free GaN epitaxial layers grown on $\mathrm{Si}(111)$ substrate by MOCVD," J. Phs. D: Appl. Phys. 41, 155217 (2008).

45 Razeghi, M. and M. Henini, Optoelectronic devices: III-Nitrides. 1st ed. San Diego: Elsevier Ltd. 2004

46 J. Blasing, A. Reiher, A. Dadgar, A. Diez, and A. Krost. "The origin of stress reduction by low-temperature AlN interlayers," Appl. Phys. Lett. 81, 2722 (2002).

47 Qian Sun, Jianfeng Wang, Hui Wang, Ruiqin Jin, Desheng Jiang, Jianjun Zhu, Degang Zhao, Hui Yang, Shengqiang Zhou, Mingfang Wu, Dries Smeets, and Andre Vantomme, "High-temperature AlN interlayer for crack-free AlGaN growth on GaN," J. Appl. Phys. 104, 043516 (2008).

48 R.Q. Jin, J.P. Liu, J.C. Zhang, H. Yang. "Growth of crack-free AlGaN film on thin AlN interlayer by MOCVD," J Crys Growth 268, 35-40 (2004)

49 C. McAleese, M. J. Kappers, F.D.G. Rayment, P. Cherns, and C.J. Humphreys. "Strain effects of AlN interlayers for MOVPE growth of crack-free AlGaN and AlN/GaN multilayers on GaN," J. Cryst. Growth 272, 475-480 (2004).

50 J. Han, K. E.Waldrip, S. R. Lee, J. J. Figiel, S. J. Hearne, G. A. Petersen, and S. M Myers. "Stress engineering during metalorganic chemical vapor deposition of AlGaN/GaN distributed Bragg reflectors," Appl. Phys. Lett. 78, 67 (2001). 
51 Ting Liu, Sandeep Chandril, A.J. Ptak, D. Korakakis, and T.H. Myers. "Bismuth surfactant effects for GaAsN and beryllium doping of GaAsN and GaInAsN grown by molecular beam epitaxy," J. Crys. Growth 304, 402-406 (2007).

52 Donghai $\mathrm{Wu}$, Zhichuan Niu, Shiyong Zhang, Haiqiao Ni, Zhenhong He, Zheng Sun, Qin Han, Ronghan $\mathrm{Wu}$. "The role of $\mathrm{Sb}$ in the molecular beam epitaxy growth of $1.30-1.55 \mathrm{~mm}$ wavelength GaInNAs/GaAs quantum well with high indium content," J. Cryst. Growth 290, 494-497 (2006).

53 Xu-Qiang Shen, Satoru Tanka, Sohachi Iwai, and Yoshinobu Aoyagi. "The formation of $\mathrm{GaN}$ dots on AlxGa12xN surfaces using Si in gas-source molecular beam epitaxy," Appl. Phys. Lett. 72, 344 (1998).

54 S. Haffouz, H. Lahreche, P. Vennegues, P. de Mierry, B. Beaumont, F. Omnes, and P. Gibart. "The effect of the $\mathrm{Si} / \mathrm{N}$ treatment of a nitridated sapphire surface on the growth mode of GaN in low-pressure metalorganic vapor phase epitaxy," Appl. Phys. Lett. 73, 1278-1280 (1998).

55 W.K. Fong, C.F. Zhu, B.H. Leung, C. Surya, B. Sundaravel, E.Z. Luo, J.B. Xu, I.H. Wilson. "Characterizations of GaN films grown with indium surfactant by RF-plasma assisted molecular beam epitaxy," Microelectronics Reliability 42, 1179-1184 (2002).

56 C.T. Foxon, S.E. Hooper, T.S. Cheng, J.W. Orton, G.B. Ren, B. Ya Ber, A. V. Merkulov, S. V. Novikov, and V. V. Tret'yakov. "Improvement of the photoluminescence from gallium nitride layers grown by MBE with an additional incident indium flux," Semicond. Sci. Technol. 13, 1469-1471 (1998).

57 Shigeo Yamaguchi, Michihiko Kariya, Takayuki Kashima, Shugo Nitta, Masayoshi Kosaki, Yohei Yukawa, Hiroshi Amano, and Isamu Akasaki. "Control of strain in GaN using an In doping-induced hardening effect," Phys. Rev. B 64, 035318 (2001).

58 Shiegeo Yamaguchi, Michihiko Kariya, Shugo Nitta, Hiroshi Amano, and Isamu Akasaki. "Strain relief by In-doping and its effect on the surface and on the interface structures in (Al) GaN on sapphire grown by metalorganic vapor-phase epitaxy," Appl. Surface Sci. 159160, 414-420 (2000).

59 O. Landre, R. Songmuang, J. Renard, E. Bellet-Amalric, H. Renevier, and B. Daudin. "Plasma-assisted molecular beam epitaxy growth of GaN nanowires using indium-enhanced diffusion" Appl. Phys. Lett. 93, 183109 (2008).

60 S. Nicolay, E. Feltin, J. F. Carlin, M. Mosca, L. Nevou, M. Tchernycheva, F. H. Julien, M. Ilegems, and N. Grandjean, "Indium surfactant effect on AlN/GaN heterostructures grown by metal-organic vapor-phase epitaxy: Applications to intersubband transitions" Appl. Phys. Lett. 88, 151902 (2006). 
${ }^{61}$ S. Keller, S. Heikman, I. Ben-yaacov, L. Shen, S. P. Denbaars, and U. K. Mishra. "Indium Surfactant Assisted Growth of AlN/GaN Heterostructures by Metal-Organic Chemical Vapor Deposition,” Phys. Stat. Sol. (a) 188, 775 (2001).

J. Song, D. Heo, W. Choi, I. Han, J. Lee, J. Kim, K. Chang, and Y. Lee. "Optical Properties of full digital-alloy InGaAlAs Multi-quantum wells and Applications to CW 200-mW 1.3$\mu \mathrm{m}$ Laser Diodes," IEEE International Semiconductor Device Research Symposium, Dec. 79, 430 (2005).

63 P. Newman, J. Pamulapati, H. Shen, M. Taysing-Lara, J. Liu, W. Chang, G. Simonis, B. Koley, M. Dagenais, S. Feld, and J. Loehr. "Molecular beam epitaxial growth of vertical cavity surface emitting lasers with digital alloys and digital gradings" J. Vac. Sci. Technol. B., 18, 1619 (2000).

64 O. Kwon, Y. Lin, J. Doeckl, and S. Ringel. "Growth and Properties of Digitally-Alloyed AlGaInP by Solid Source Molecular Beam Epitaxy” J. Electronic Materials, 34, 1301 (2005).

65 C. Mourd, D. Gianardi, K. Malloy, and R. Kaspi. "2 $\mu \mathrm{m}$ GaInAsSb/AlGaAsSb midinfrared laser grown digitally on GaSb by modulated-molecular beam epitaxy," J. Appl. Phys. 88, $5543(2000)$.

66 M. Asif Khan, J. N. Kuznia, D. T. Olson, T. George, and W. T. Pike. "GaN/AlN digital alloy short-period superlattices by switched atomic layer metalorganic chemical vapor deposition”Appl Phys. Lett. 63, 3470 (1993).

67 P. K. Kandaswamy, C. Bougerol, D. Jalabert, P. Ruterana, and E. Monroy. "Strain relaxation in short-period polar GaN/AlN superlattices," J. Appl. Phys. 106, 013526 (2009).

68 J. Zhang, E. Kuokstis, Q. Fareed, H. Wang, J. Yang, G. Simin, M. Asif Khan, R. Gaska, and M. Shur. "Pulsed atomic layer epitaxy of quaternary AlInGaN layers,"Appl. Phys. Lett. 79, $925(2001)$.

69 Tomoyuki Miyamoto, Shunichi Sato, Zhong Pan, Dietmar Schlenker, Fumio Koyama, and Kenichi Iga. "GaNAs/GaInAs short-period superlattice quantum well structures grown by MOCVD using TBAs and DMHy”J. Cryst. Growth. 195, 421-426 (1998).

70 Makoto Kurimoto, Toshiyuki Nakada, Yoshiyuki Ishihara, Masao Shibata, Tohru Honda, and Hideo Kawanishi. "Tensile Strain Introduced in AlN Layer Grown by Metal-Organic VaporPhase Epitaxy on (0001) 6H-SiC with (GaN/AlN) Buffer," Jpn. J. Appl. Phys. 38, L551L553 (1999).

71 Eric Feltin, B. Beaumont, M. Laugt, P. de Mierry, P. Vennegues, H. Lahreche, M. Lerous, and P. Gibart. "Stress control in GaN grown on silicon (111) by metalorganic vapor phase epitaxy,” Appl. Phys. Lett. 79, 3230 (2001). 
72 Yu-Li Tsai, Cheng-Liang Wang, Po-Hung Lin, Wei-Tsai Liao, and Jyh-Rong Gong, "Observation of compositional pulling phenomenon in $\mathrm{AlGaN}(0.4<\mathrm{x}<1.0)$ films grown on (0001) sapphire substrates" Appl. Phys. Lett. 82, 31 (2003).

73 A. D. Bykhovski, B. L. Gelmont, and M. S. Shur, "Elastic strain relaxation and piezoeffect in GaN-AlN, GaN-AlGaN and GaN-InGaN superlattices”J. Appl. Phys. 81, 6332 (1997).

74 Michael A. Mastro, Joshua D. Caldwell, Ron T. Holm, Rich L. Henry, and Charles R. Eddy Jr. "Design of Gallium Nitride Resonant Cavity Light-Emitting Diodes on $\mathrm{Si}$ Substrates,"Adv. Mater. 20, 115 (2008).

75 T. Someya and Y. Arakawa. "Highly reflective GaN/AlGaN quarter-wave reflectors grown by metal organic chemical vapor deposition" Appl. Phys. Lett. 73, 3653 (1998).

76 G. S. Huang, T. C. Lu, H. H. Yao, H. C. Kuo, S. C. Wang, Chih-Wei Lin, and Li Chang. "Crack-free GaN/AlN distributed Bragg reflectors incorporated with GaN/AlN superlattices grown by metalorganic chemical vapor deposition.” Appl. Phys. Lett. 88, 061904 (2006).

77 H.M. Ng, T. D. Moustakas, and S. N. G. Chu. "High Reflectivity and broad bandwidth AlN/GaN distributed Bragg reflectors grown by molecular-beam epitaxy," Appl. Phys. Lett. 76, 2818 (2000).

78 H. S. Cheong, T. V. Cuong, H. G. Kim, J. Y. Park, C. S. Kim, C. H. Hong, J. H. Baek, S. H. Lee, T. M. Kim, and Y. M. Yu, "Suppression of cracks and V-shaped defects, and improvement of reflectivity of GaN/AlGaN distributed Bragg reflectors by insertion of multiple interlayers," Phys. Stat. Sol. (a) 201, 2799 (2004).

79 J. F. Carlin, C. Zellweger, J. Dorsaz, S. Nicolay, G. Christmann, E. Feltin, R. Butte, and N. Grandjean. "Progresses in III-Nitride distributed Bragg reflectors and microcavities using AlInN/GaN materials," Phys. Stat. Sol. B 242, 2326-2344 (2005).

80 R. Butté, J-F Carlin, E. Feltin, M. Gonschorek, S. Nicolay, G. Christmann, D. Simeonov, A. Castiglia, J. Dorsaz, H. J. Buehlmann, S. Christopoulos, G. Baldassarri Hoger von Hogersthal, A. J. D. Grundy, M. Mosca, C. Pinquier, M. A. Py, F. Demangeot, J. Frandon, P. G. Lagoudakis, J. J. J. Baumberg, and N. Grandjean. "Current status of AlInN layers latticematched to GaN for photonics and electronics," J. Phys. D: Appl. Phys. 40, 6328-6344 (2007).

81 R. Butte, E. Feltin, J. Dorsaz, G. Christmann, J. Carlin, N. Grandjean, M. Ilegems. "Recent Progress in the Growth of Highly Reflective Nitride-Based Distributed Bragg Reflectors and Their Use in Microcavities,” Jpn. J. Appl. Phys. 44, 7207 (2005).

82 J. Dorsaz, J. Carlin, S. Gradecak, and M. Ilegems. "Progress in AlInN-GaN Bragg reflectors: Application to a microcavity light emitting diode," J. Appl. Phys. 97, 084505 (2005). 
83 R. Singh, D. Doppalapudi, T. Moustakas, and L. Romano. "Phase separation in InGaN thick films and formation of InGaN/GaN double heterostructures in the entire alloy composition," Appl. Phys. Lett. 70, 1089 (1997).

D. Korakakis, K. Ludwig, and T. Moustakas. "Long range order in $\mathrm{Al}_{\mathrm{x}} \mathrm{Ga}_{1-\mathrm{x}} \mathrm{N}$ films grown by molecular beam epitaxy,” Appl. Phys. Lett. 71, 71 (1997).

D. Doppalpudi, S. Basu, K. Ludwig, and T. Moustakas. "Phase separation and ordering in InGaN alloys grown by molecular beam epitaxy,” J. Appl. Phys. 84, 1389 (1998).

N. M. Ravindra, B. Sopori, O. H. Gokce, S. X. Cheng, A. Shenoy, L. Jin, S. Abedrabbo, W. Chen, and Y. Zhang. "Emissivity Measurements and Modeling of Silicon-Related Materials: An Overview," International Journal of Thermophysics, 22, 1593 (2001).

M. A. Moram and M. E. Vickers. "X-Ray Diffraction of III-Nitrides," Rep. Prog. Phys. 72, 036502 (2009).

R. Swanepoel. "Determination of the tchikness and optical constants of amorphous silicon," J. Phys. E: Sci. Instrum. 16, 1215 (1983).

B. E. A. Saleh and M. C. Teich. Fundamentals of Photonics. New Jersey:John Wiley \& Sons (2007).

D. Goldstein. "Polarized Light, Second Edition" CRC Oress, (2003).

91 J. A. Woollam Co., Inc., “A Short Course in Ellipsometry,” (2001).

Ben G. Streetman and Sanjay Banerjee, Solid State Electronic Devices, New Jersey:Prentice Hall (2000).

National Institute of Science and Technology. "Hall Effect Measurements," $<$ http://www.nist.gov/pml/semiconductor/hall.cfm>

H. M. Ng, D. Doppalapudi, T. D. Moustakas, N. G. Weimann, and L. F. Eastman. "The role of dislocation scattering in n-type GaN films," Appl. Phys. Lett. 73, 821 (1998).

L. T. Romano, C. G. Van de Walle, J. W. Ager III, W. Gotz, and R. S. Kern, "Effect of Si doping on strain, cracking, and microstructure in $\mathrm{GaN}$ thin films by metalorganic chemical vapor deposition," J. Appl. Phys. 87, 7745 (2000).

E. L. Waldron, Y.L. Li, and E. F. Schubert, "Experimental study of perpendicular transport in weakly coupled AlxGa1-xN/GaN superlattices,” Appl. Phys. Lett. 83, 4975 (2003).

97 Uttiya Chowdhury, Raymond K. Price, Michael M. Wong, Dongwon Yoo, Xuebing Zhang, Milton Feng, and Russell D. Dupuis. "Modulation-doped superlattice AlGaN barrier GaN/AlGaN HFETs," J. Cryst. Growth 272, 318-321 (2004).

L. E. Rodak and D. Korakakis, "Properties of Aluminum Gallium Nitride Digital Alloy Growth via Metal Organic Vapor Phase Epitaxy," J. Electron. Mater. 40, 388 (2011). 
99 L. E. Rodak and D. Korakakis, "Properties of Digital Aluminum Gallium Nitride Alloys Grown via Metal Organic Vapor Phase Epitaxy," Mat. Res. Soc. Symp. Proc., 1202, 1202I05-05 (2010).

100 Harold P. Klug and Leroy E. Alexander. X-Ray Diffraction Procedures, WileyInterscience:New York (1974).

${ }^{101}$ Stephen J. Pearton, ed, Optoelectronic Properties of Semiconductors and Superlattices - GaN and Related Materials. Canada: Gordon and Breach Science Publishers (2000).

${ }^{102}$ A. Chandolu, S. Nikishin, M. Holtz, and H. Temkin. "X-ray diffraction study of AlN/AlGaN short period superlattices." J. Appl. Phys. 102, 114909 (2007).

${ }^{103}$ Yoshihiro Kida, Tomohiko Shibata, Hideto Miyake, and Kazumasa Hiramatsu. "Metalorganic Vapor Phase Epitaxy Growth and Study of Stress in AlGaN Using Epitaxial AlN as Underlying Layer,” Jpn. J. Appl. Phys. 42, L572 (2003).

${ }^{104}$ Aurelien David, Tetsuo Fujii, Rajat Sharma, Kelly McGroddy, Shuji Nakamura, Steven P. DenBaars, Evelyn L. Hu, Claude Weisbuch, and Henri Benisty. "Photonic-crystal GaN lightemitting diodes with tailored guided modes distribution," Appl. Phys. Lett. 88, 061124 (2006).

${ }^{105}$ S. Einfeldt, V. Kirchner, H. Heinke, M. DieBelberg, S Figge, K. Vogeler, and D. Hommel. "Strain relaxation in AlGaN under tensile plane stress." J. Appl. Phys. 88, 7029 (2000).

${ }^{106}$ L. E. Rodak, C. M. Miller, and D. Korakakis, "Surfactant Effects of Indium on Cracking in AlN/GaN Distributed Bragg Reflectors Grown via Metal Organic Vapor Phase Epitaxy," Superlattices Microstruct., 49, 52-59 (2011).

${ }^{107}$ L. E. Rodak, C. M. Miller, and D. Korakakis. "Surfactant Effects of Indium in the Growth of AlN/GaN Distributed Bragg Reflectors via Metal Organic Vapor Phase Epitaxy," Mat. Res. Soc. Symp. Proc., 1195, 1195-B10-01 (2010).

108 L. E. Rodak and D. Korakakis. "Effect of In as surfactant on the growth of AlN/GaN Distributed Bragg Reflectors by Metal Organic Vapor Phase Epitaxy," Mat. Res. Soc. Symp. Proc. 1108, 1108-A09-18 (2009).

${ }^{109}$ S. Einfeldt, M. Dießelberg, H. Heinke, D. Hommel, D. Rudloff, J. Christen, and R. F. Davis. "Strain in cracked AlGaN layer." J. Appl. Phys. 92, 118 (2002).

${ }^{110}$ H. M. Ng, D. Doppalapudi, E. IIiopoulos, and T. D. Moustakas. "Distributed Bragg reflectors based on AlN/GaN multilayers," Appl. Phys. Lett. 74, 1036 (1999).

${ }^{111}$ B. Schineller and M. Heuken. "Recent advances in MOCVD process technology for the growth of compound semiconductor devices." Appl. Phys. A 87, 479 (2007). 
112 R.A. Talalaev, E.V. Yakovlev, Yu.N. Makarov, B.S. Yavich, and W.N. Wang. "Modeling and experimental analysis of GaN MOVPE in AIX 200 RF reactor," PS.VI.05, in: 10th European Workshop of MOVPE (2003).

${ }^{113}$ E. Northrup and C. G. Van de Walle. "Indium versus hydrogen-terminated GaN (0001) surfaces: Surfactant effect of indium in a chemical vapor deposition environment." Appl. Phys. Lett. 84, 4322 (2004).

114 J. Neugebauer. "Surfactants and antisurfactants on group-III-nitride surfaces." Phys. Stat. Sol. (c) 0, 1651, (2003).

${ }^{115}$ Z. H. Feng, S. J. Cai, K. J. Chen, and Kei May Lau, Appl. Phys. Lett. "Isoelectronic indiumsurfactant-doped $\mathrm{Al}_{0.3} \mathrm{Ga}_{0.7} \mathrm{~N} / \mathrm{GaN}$ high electron mobility transistors." 88, 122113 (2006).

${ }^{116}$ T. M. Al tahtamouni, A. Sedhain, J. Lin, and H. X. Jiang, "Si-doped high Al-content AlGaN epilayers with improved quality and conductivity using indium as a surfactant." Appl. Phys. Lett. 92, 092105 (2008).

${ }^{117}$ Eugene Hecht. Optics. Massachusetts:Addison-Wesley Publishing (1990).

${ }^{118}$ H. A. Macleod, Thin Film Optical Filters. New York:McGraw-Hill (1989).

${ }^{119}$ G.M. Laws, E.C. Larkins, I. Harrison, C. Molloy, and D. Somerford. "Improved refractive index formulas for the $\mathrm{Al}_{x} \mathrm{Ga}_{1-\mathrm{x}} \mathrm{N}$ and $\mathrm{In}_{\mathrm{y}} \mathrm{Ga}_{1-\mathrm{y}} \mathrm{N}$ alloys." J. Appl. Phys. 89, 1108 (2001).

120 Robert E. Newnham. Properties of Materials: Anisotropy, Symmetry, Structure. New Your:Oxford Press (2005).

${ }^{121}$ S. Pezzagna, J. Brault, M. Leroux, J. Massies, and M. De Micheli. "Refractive indices and elasto-optic coefficients of GaN studied by optical waveguiding.” J. Appl. Phys. 103, 123112 (2008).

${ }^{122}$ S.H. Wemple and M. DrDomenico. "Theory of the Elasto-Optic Effect in Nonmetallic Crystals.” Phys. Rev. B 1, 193 (1970).

${ }^{123}$ E. Bellet-Amalric, C. Adelmann, E. Sarigiannidou, J. L. Rouviere, G. Feuillet, E. Monroy, and B. Daudin, "Plastic strain relaxation of nitride heterostructures." J. Appl. Phys. 95, 1127 (2004).

${ }^{124}$ Eugene Hecht. Optics. Massachusetts:Addison-Wesley Publishing (1990).

${ }^{125}$ Sadao Adachi, Peter Capper, and Safa Kasap. Properties of Semiconductor Alloys - Group IV, III-V, and II-VI Semiconductors, United Kingdom:John Wiley \& Sons Ltd (2009).

${ }^{126}$ Y.F. Li, B. Yao, Y.M. Lu, Y.Q. Gai, C.Z. Cong, Z.Z. Zhang, D.X. Zhao, J.Y. Zhang, B.H. Li, D.Z.Shen, X.W. Gan, and Z.K. Tang. "Biaxial stress-dependent optical band gap, crystalline, and electronic structure in wurtzite $\mathrm{ZnO}$ : Experimental and ab initio study." J. Appl. Phys. 104, 083516 (2008). 
${ }^{127}$ S. Yu. Davydov. Semicoductors. "Evaluation of Physical Parameters for the Group III Nitrates: BN, AlN, GaN, and InN." 36, 41 (2002).

${ }^{128}$ S. Einfeldt, H. Heinke, V. Kirchner, and D. Hommel. "Strain relaxation in AlGaN/GaN superlattices grown on GaN.” J. Appl. Phys. 89, 2160 (2001).

${ }^{129}$ E. Dogheche, P. Ruterana, G. Nouet, F. Omnes, and P. Gibart. "Investigation of Refractive Index and Optical Propagation Loss in Gallium Nitride Based Waveguides." Phys. Stat. Sol. (b) 228, 429 (2001).

${ }^{130}$ J. N. Hilfiker, C. L. Bungay, R. A. Synowicki, T. E. Tiwald, C. M. Herzinger, B. Johs, G. K. Pribil, and J. A. Woollam. "Progress in spectroscopic ellipsometry: Applications from vacuum ultraviolet to infrared." J. Vac. Sci. Technol. A. 21, 1103 (2003).

${ }^{131}$ K. Pakula, A. Wysmolek, K.P. Korona, J.M. Baranowski, R. Stepniewski, I. Grzegory, M. Bockowski, J. Jun, S. Krukowski, M. Wroblewski, and S. Porowski. "Luminescence and Reflectivity in the Exciton Region of Homoepitaxial GaN Layers Grown on GaN Substrates." Solid State Communications 97, 919 (1996).

${ }^{132}$ K. Lee, L. E. Rodak, V. Kumbham, V. Narang, L. A. Hornak, and D. Korakakis, "InGaN MQW LED Structures Using AlN/GaN DBR and Ag-based P-contact," Mater. Res. Soc. Symp. Proc., 1288, mrsf10-1288-g11-21 (2011).

${ }^{133}$ V. Kumbham, Sridhar Kuchibhatla, K. Lee, L. E. Rodak, V. Narang, D. Korakakis, and L. A. Hornak, "Ohmic and Highly Reflective Ag Based Contact on p-GaN for RCLED," Mater. Res. Soc. Symp. Proc., 1288, mrsf10-1288-g11-11 (2011).

${ }^{134}$ Mary H. Crawford. "LEDs for Solid-State Lighting: Performance Challenges and Recent Advances." IEEE J. Sel. Top. Quantum Electron.15, 1028 (2008).

${ }^{135}$ Chuen-Ching Wang, Wen-Ran Yang, Jin-Jia Chen, and Wei-Wen Shi. “A Modified Flip-Chip LED Packaging Design With Enhanced Light Coupling Efficiency for Plastic Optical Fiber Networks.” J. Electron. Packag. 131,041002 (2009).

${ }^{136}$ H. W. Choi, C. Liu, E. Gu, G. McConnell, J. M. Girkin, I. M. Waston, and M. D. Dawson. "GaN micro-light-emitting diode arrays with monolithically integrated sapphire microlenses.” Appl. Phys. Lett. 84, 2253 (2004).

137 Jae-Jun Kim, Sunki Chae, and Ki-Hun Jeong. "Micropatterned single lens for wide-angle light-emitting diodes." Opt. Lett. 35, 823 (2010).

${ }^{138}$ Chun-Geng Lai, Hao-Chung Kuo, Peichen Yu, Tien-Chang Lu, Chia-Hsin Chao, Hsi-Hsuan Yen, and Wen-Yung Yeh. "Highly-directional emission patterns based on near single guided mode extraction from GaN-based ultrathin microcavity light-emitting diodes with photonic crystals.” Appl. Phys. Lett. 97, 013108 (2010). 
${ }^{139}$ S. Srinivasan, M. Stevens, F. A. Ponce, and T. Mukai. "Polychromatic light emission from single InGaN quantum wells grown on pyramidal GaN facets." Appl. Phys. Lett. 87, 131911 (2005).

${ }^{140}$ Isaac H. Wildeson, Robert Colby, David A. Ewoldt, Zhiwen Liang, Dmitri N. Zakharov, Nestor J. Zaluzec, R. Edwin Garcia, Eric A. Stach, and Timothy D. Sands. "III-nitride nanopyramid light emitting diodes grown by organometallic vapor phase epitaxy." J. Appl. Phys. 108, 044303 (2010).

141 Mitsuru Funato, Masaya Ueda, Yoichi Kawakami, Yukio Narukawa, Takao Kosugi, Masayoshi Takahashi, and Takashi Nukai. "Blue, Green, and Amber InGaN/GaN LightEmitting Diodes on Semipolar $\{1122\}$ GaN Bulk Substrates.” Jap. J. Appl. Phys. 45, L659 (2006).

${ }^{142}$ L. E. Rodak, K. Lee, V. Kumbham, V. Narang, L. A. Hornak, and D. Korakakis, "Light Emitting Diode Growth on Curved GaN Surfaces," Mater. Res. Soc. Symp. Proc., 1288, mrsf10-1288-g06-30 (2011).

${ }^{143}$ P. Gibart. "Metal organic vapour phase epitaxy of GaN and lateral overgrowth." Rep. Prog. Phys. 67, 667 (2004).

${ }^{144}$ X. Li, A. M. Jones, S. D. Roh, D. A. Turnbull, S. G. Bishop, and J. J. Coleman. "Characteristics of GaN Stripes Grown by Selective-Area Metalorganic Chemical Vapor Deposition.” J. Electron. Mater. 26, 306 (1997).

${ }^{145}$ L. E. Rodak, K. R. Kasarla, D. Korakakis, "Effect of Gas Flow on the Selective Area Growth of Gallium Nitride via Metal Organic Vapor Phase Epitaxy,” J. Crystal Growth, 306, 75-79 (2007).

${ }^{146}$ L. E. Rodak, N. J. Berry Ann, Kalyan Reddy Kasarla, Nanying Yang, D. Korakakis, "Study of ELOG GaN for Application in the Fabrication of Micro-channels for Optoelectronic Devices," Mater. Res. Soc. Symp. Proc., 892 (2006).

${ }^{147}$ N. J. Berry Ann, L. E. Rodak, K. R. Kasarla, N. Yang, D. Korakakis, "Study of ELOG GaN for application in the fabrication of optoelectronic devices," Proceedings of SPIE Vol. 6017, 60170D1-8 (2005).

148 Kazumasa Hiramatsu, Hideto Miyake. "Review of Facet Controlled Epitaxial Lateral Overgrowth (FACELO) of GaN via Low Pressure Vapor Phase Epitaxy." Mat. Res. Soc. Symp. Proc. Vol. 639 (2001).

${ }^{149}$ M. E. Coltrin, C. C. Mitchel, "Kinetics and Transport in Gallium Nitride Epitaxial Lateral Overgrowth." in III-Nitride Based Semiconductor Electronic and Optical Devices and Stateof-the-Art Program on Compound Semiconductors XXXIV, F. Ren, D. N. Buckley, S. N. G. Chu, S. J. Pearton, Editors, PV 2001-01, p. 1, The Electrochemical Society Proceedings Series, Pennington, NJ (2001). 
${ }^{150}$ K. Harafuji. "Transport of Gas-Phase Species Stored in Stagnant Volumes under a GaN Metalorganic Vapor Phase Epitaxy Horizontal Reactor.” Jpn. J. Appl. Phys. 40, 6263 (2001).

${ }^{151}$ X.M. Shen, G. Feng, B.S. Zhang, L.H. Duan, Y.T. Wang, H. Yang. "Selective area growth of GaN on GaAs (001) substrates by metalorganic vapor-phase epitaxy." J. Crystal Growth, 252, 9 (2003).

152 J. Olivares, E. Iborra, M. Clement, L. Vergara, J. Sangrador, and A. Sanz-Hervás, "Piezoelectric actuation of microbridges using AlN." Sensors and Actuators A 123-124, 590595 (2005).

${ }^{153}$ G. Yaralioglu, M. Badi, A. Ergun, C. Cheng, B. Khuri-Yakub, and F. Degertekin, "Lamb wave devices using capacitive micromachined ultrasonic transducers." Appl. Phys. Lett.78, 111-113 (2001).

${ }^{154}$ E. Zaus, M. Hermann, M. Stutzmann, and M. Eickhoff, "Fabrication of freestanding GaN microstructures using AlN sacrificial layers.” Phys. Stat. Sol. (RRL) 1, R10-R12 (2007).

${ }^{155}$ N. Nguyen, X. Huang, and T. Chuan, "MEMS-Micropumps: A Review." Trans. Am. Soc. Mech. Eng. 124, 384-391 (2002).

${ }^{156}$ R. Duggirala and A. Lal, J. "A Hybrid PZT-Silicon Microvalve.” Microelectromech. Sys. 14, 488-497 (2005).

157 J. R. Mileham, S. J. Pearton, C. R. Abernathy, J. D. MacKenzie, R. J. Shul and S. P. Kilcoyne. "Wet chemical etching of AlN." Appl. Phys. Lett. 67, 1119-1121 (1995).

${ }^{158}$ I. Cimalla, Ch. Foerster, V. Cimalla, V. Lebedev, D. Cengher, and O. Ambacher. "Wet chemical etching of AlN in KOH solution.” Phys. Stat. Sol. (c) 3, 1767-1770 (2006).

${ }^{159}$ J. Olivares, M. Clement, E. Iborra, L. Vergara, J. L. Sanchez-Rojas, J. Vazquez, and P. Sanz. "Simulation, fabrication, and testing of aluminium nitride piezoelectric microbridges." Smart Sensors, Actuators, and MEMS II, Proc. of SPIE 5836, 16-26 (2005).

${ }^{160}$ S. Saravanan , E. Berenschot, G. Krijnen, M. Elwenspoek. "A novel surface micromachining process to fabricate AlN unimorph suspensions and its application for RF resonators." Sensors and Actuators A 130-131, 340-345 (2006).

161 L. E. Rodak, Sridhar Kuchibhatla, and D. Korakakis. "Suspended Aluminum Nitride Structures Grown via Metal Organic Vapor Phase Epitaxy,” Mater. Lett., 63, 1571 (2009).

162 Sridhar Kuchibhatla, L. E. Rodak, and D. Korakakis, "Fourier Transform Infrared Spectroscopy of AlN Thin Films Grown on Sacrificial Silicon Oxide Layers via Metal Organic Vapor Phase Expitaxy", Thin Solid Films 519, 117-121 (2010).

${ }^{163}$ L. E. Rodak, Sridhar Kuchibhatla, P. Famouri, Ting Liu, and D. Korakakis. "Aluminum Nitride Micro-Channels Grown via Metal Organic Vapor Phase Epitaxy for MEMs Applications,” Mat. Res. Soc. Symp. Proc. 1040, 1040-Q09-28 (2008). 
${ }^{164}$ Sridhar Kuchibhatla, L. E. Rodak, and D. Korakakis, "Investigating the stress and crystal quality of AlN Air-Bridges through Micro-Raman scattering," Mat. Res. Soc. Symp. Proc. 1139, 1139-GG03-46 (2009).

${ }^{165}$ Sridhar Kuchibhatla, L. E. Rodak, and D. Korakakis, "Characterization of Aluminum Nitride (AlN) thin films through micro infrared spectroscopy," Mat. Res. Soc. Symp. Proc. 1222, 1222-DD02-28 (2010).

${ }^{166}$ K. Tonisch, F. Niebelschuetz, V. Cimalla, H. Romanus, and O. Ambacher. "Fully Unstrained GaN on Thick AlN Layers for MEMS Application.” Mater. Res. Soc. Symp. Proc. 955, I1603 (2007).

167 A. R. Stonas, N. C. MacDonald, K. L. Turner, S. P. DenBaars and E. L. Hu. "Photoelectrochemical undercut etching for fabrication of $\mathrm{GaN}$ microelectromechanical systems.” J. Vac. Sci. Technol. B 19, 2838-2841 (2001).

168 J. Bühler, F. Steiner, and H. Baltes. "Silicon dioxide sacrificial layer etching in surface micromachining." J. Micromech Microeng. 7, R1-R13 (1997).

${ }^{169}$ S. J. Pearton, C. R. Abernathy, F. Ren, and J. R. Lothian, P. W. Wisk, and A. Katz. "Dry and wet etching characteristics of $\mathrm{InN}, \mathrm{AlN}$, and $\mathrm{GaN}$ deposited by electron cyclotron resonance metalorganic molecular beam epitaxy.” J. Vac. Sci. Technol. A 11, 1772-1775 (1993).

${ }^{170}$ T. Katona, P. Cantu, S. Keller, Y. Wu, J. Speck, and S. DenBaars. "Maskless lateral epitaxial overgrowth of high-aluminum-content $\mathrm{Al}_{\mathrm{x}} \mathrm{Ga}_{1-\mathrm{x}} \mathrm{N}$.” Appl. Phys. Lett. 84, 5025-5027 (2004).

${ }^{171}$ Y. Kwaguchi, G. Sugahara, A. Mochida, T. Shimamoto, A. Ishibashi, and Y. Yokogawa, "Low-dislocation density AlGaN layer by air-bridged lateral epitaxial growth." Phys. Stat. Sol. (C) 0, 2107-2110 (2003).

${ }^{172}$ Chih-Chien Lin and Ching-Ting Lee. "GaN-Based Resonant-Cavity Light Emitting Diodes With Top and Bottom Dielectric Distributed Bragg Reflectors," IEEE Photonics Technology Letters, 22, 1291 (2010).

${ }^{173}$ M. Kneissl, T. Kolbe, C. Chua, V. Kueller, N. Lobo, J. Stellmach, A. Knauer, H. Rodriguex, S. Einfeldt, Z. Yang, N. M. Johnson, and M. Wyers. "Advances in group III-nitride-based deep UV light-emitting diode technology,” Semicond. Sci. Technol. 26, 014035 (2011).

${ }^{174}$ Sergey Nikishin, Boris Borisov, Vladimir Kuryatkov, Mark Holtz, Gregory Garrett, Wendy Sarney, Anand Sampath, Hongen SHen, Micheal Wraback, Alexander Usikov, and Vladimir Dmitriev. J. Mater. Sci: Mater electron 19, 764 (2008).

${ }^{175}$ A. A. Allerman, M. H. Crawford, M. A. Miller, and S. R. Lee. "Growth and characterization of Mg-doped AlGaN-AlN short-period superlattices for deep-UV optoelectronic devices" J. Cryst. Growth 312, 756 (2010). 
${ }^{176}$ Lei Wang, Rui Li, Ding Li, Ningyang Liu, Lei Liu, Weihua Chen, Cunda Wang, Zhijian Yang, and Xiaodong Hu. "Strain modulation-enhanced Mg acceptor activation efficiency of $\mathrm{Al}_{0.14} \mathrm{Ga}_{0.86} \mathrm{~N} / \mathrm{GaN}$ superlattices with AlN interlayer," Appl. Phys. Lett. 96, 061110 (2010).

${ }^{177}$ Peter Kozodoy, Monica Hansen, Steven P. DenBaars, and Umesh K. Mishra. "Enhanced Mg doping efficiency in A10.2Ga0.8N/GaN superlattices,” Appl. Phys. Lett. 74, 3681 (1999). 